Supporting Information

\title{
Iron-Catalyzed Oxidative Coupling of Indoline-2-ones with Aminobenzamides via Dual C-H Functionalization
}

\author{
Yi-Huan Lai,ł Run-Shi Wu, $\$$ Jie Huang, Jun-Yu Huang, and Da-Zhen Xu* \\ National Engineering Research Center of Pesticide (Tianjin), State Key Laboratory and \\ Institute of Elemento-Organic Chemistry, College of Chemistry, Nankai University, Tianjin \\ 300071, China, E-mail: xudazhen@nankai.edu.cn \\ \$These authors contributed equally.
}

\section{Table of contents}

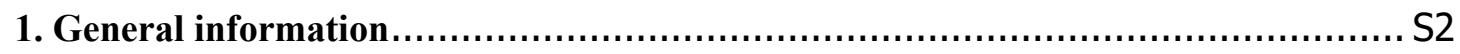

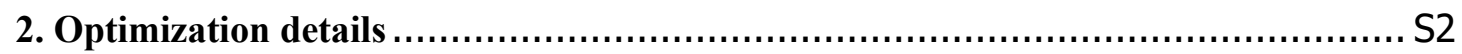

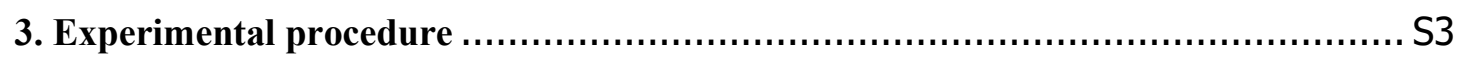

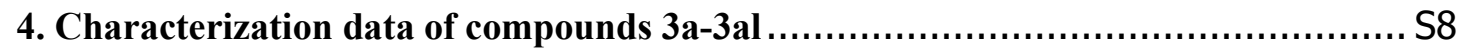

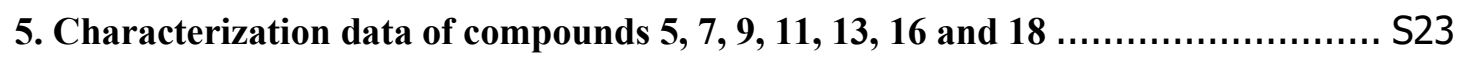

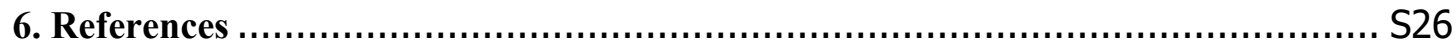

7. Spectroscopic data for compounds 3a-3al ........................................ S28

8. Spectroscopic data for compounds $5,7,9,11,13,16$ and $18 \ldots \ldots \ldots \ldots \ldots \ldots \ldots . . . \ldots 102$ 


\section{General information}

All chemicals were purchased from commercial suppliers and were used without further purification. Melting points were determined with an X-4 apparatus and are uncorrected. ${ }^{1} \mathrm{H}$ NMR and ${ }^{13} \mathrm{C}$ NMR spectra were recorded on a Bruker AV-400 spectrometer with DMSO- $d_{6}$ as the solvent. Chemical shifts are reported relative to TMS as internal standard. The ${ }^{1} \mathrm{H}$ NMR data are reported as the chemical shift in parts per million, multiplicity ( $\mathrm{s}$, singlet; $\mathrm{d}$, doublet; $\mathrm{t}$, triplet; $\mathrm{m}$, multiplet), coupling constant in hertz, and number of protons. HRMS were obtained on an IonSpec FT-ICR mass spectrometer with ESI resource.

\section{Optimization details}

\subsection{Optimization of the reaction conditions}

Table S1. Optimization of the reaction conditions. ${ }^{[a]}$

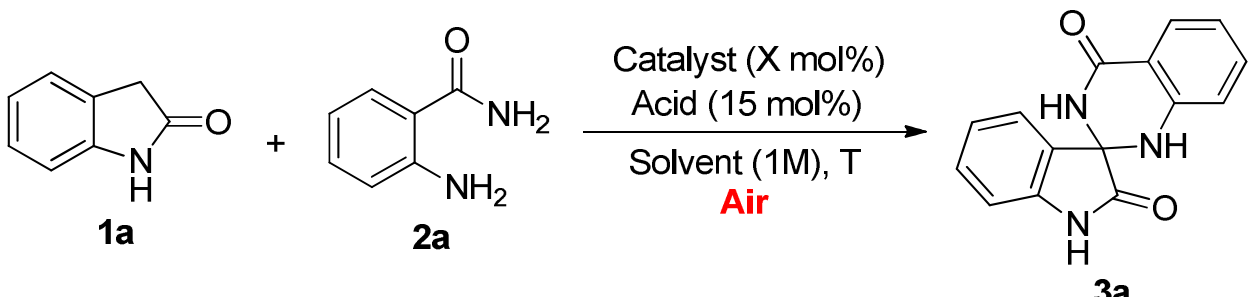

$3 a$

\begin{tabular}{|c|c|c|c|c|c|c|}
\hline Entry & Cat (10 mol\%) & Acid (15 mol\%) & $\begin{array}{l}\text { Solvent } \\
(\mathrm{mL})\end{array}$ & $\mathrm{T}\left({ }^{\circ} \mathrm{C}\right)$ & $\begin{array}{l}\text { Time } \\
\text { (h) }\end{array}$ & $\begin{array}{l}\text { Yield } \\
(\%)^{[b]}\end{array}$ \\
\hline 1 & $\mathrm{FeCl}_{3} \cdot 6 \mathrm{H}_{2} \mathrm{O}$ & --- & $\mathrm{DMF}$ & 80 & 5 & 42 \\
\hline 2 & $\mathrm{FeCl}_{3} \cdot 6 \mathrm{H}_{2} \mathrm{O}$ & $p$-TSA & DMF & 80 & 5 & 68 \\
\hline 3 & --- & $p$-TSA & DMF & 80 & 5 & --- \\
\hline 4 & $\mathrm{FeCl}_{3}$ & $p$-TSA & DMF & 80 & 5 & 83 \\
\hline 5 & $\mathrm{FeBr}_{3}$ & $p$-TSA & DMF & 80 & 5 & 69 \\
\hline 6 & $\mathrm{Fe}_{2}\left(\mathrm{SO}_{4}\right)_{3} \cdot \mathrm{xH}_{2} \mathrm{O}$ & $p$-TSA & DMF & 80 & 5 & 81 \\
\hline 7 & $\mathrm{Fe}\left(\mathrm{NO}_{3}\right)_{3} \cdot 9 \mathrm{H}_{2} \mathrm{O}$ & $p$-TSA & DMF & 80 & 5 & 74 \\
\hline 8 & $\mathrm{FeCl}_{2} \cdot 4 \mathrm{H}_{2} \mathrm{O}$ & $p$-TSA & DMF & 80 & 5 & 60 \\
\hline 9 & $\mathrm{Cu}(\mathrm{OAc})_{2} \cdot \mathrm{H}_{2} \mathrm{O}$ & $p$-TSA & DMF & 80 & 5 & --- \\
\hline 10 & $\mathrm{FeCl}_{3}$ & $p$-TSA & DMSO & 80 & 5 & 76 \\
\hline 11 & $\mathrm{FeCl}_{3}$ & $p$-TSA & $\mathrm{C}_{2} \mathrm{H}_{5} \mathrm{OH}$ & 80 & 5 & 19 \\
\hline 12 & $\mathrm{FeCl}_{3}$ & $p$-TSA & dioxane & 80 & 5 & 21 \\
\hline 13 & $\mathrm{FeCl}_{3}$ & $p$-TSA & $\mathrm{PhCl}$ & 80 & 5 & 24 \\
\hline 14 & $\mathrm{FeCl}_{3}$ & $p$-TSA & $\mathrm{H}_{2} \mathrm{O}$ & 80 & 5 & 20 \\
\hline 15 & $\mathrm{FeCl}_{3}$ & $p$-TSA & DMF & 120 & 5 & 37 \\
\hline 16 & $\mathrm{FeCl}_{3}$ & $p$-TSA & DMF & 100 & 5 & 70 \\
\hline 17 & $\mathrm{FeCl}_{3}$ & $p$-TSA & DMF & 50 & 16 & 63 \\
\hline 18 & $\mathrm{FeCl}_{3}$ & $p$-TSA & DMF & 30 & 16 & 61 \\
\hline $19^{[\mathrm{c}]}$ & $\mathrm{FeCl}_{3}(5)$ & $p$-TSA & DMF & 80 & 7 & 81 \\
\hline
\end{tabular}




\begin{tabular}{|c|c|c|c|c|c|}
\hline $20^{[\mathrm{d}]}$ & $\mathrm{FeCl}_{3}(20)$ & $p$-TSA & DMF & 80 & 5 \\
\hline $21^{[\mathrm{e}]}$ & $\mathrm{FeCl}_{3}(50)$ & $p$-TSA & $\mathrm{DMF}$ & 80 & 5 \\
\hline $22^{[\mathrm{f}]}$ & $\mathrm{FeCl}_{3}(100)$ & $p$-TSA & DMF & 80 & 5 \\
\hline $23^{[\mathrm{g}]}$ & $\mathrm{FeCl}_{3}$ & $p$-TSA (5) & $\mathrm{DMF}$ & 80 & 5 \\
\hline $24^{[\mathrm{h}]}$ & $\mathrm{FeCl}_{3}$ & $p$-TSA (30) & DMF & 80 & 5 \\
\hline $25^{[\mathrm{i}]}$ & $\mathrm{FeCl}_{3}$ & $p$-TSA (50) & $\mathrm{DMF}$ & 80 & 5 \\
\hline 26 & $\mathrm{FeCl}_{3}$ & $\begin{array}{l}\text { L-Camphorsulfon } \\
\text { ic acid }\end{array}$ & $\mathrm{DMF}$ & 80 & 5 \\
\hline 27 & $\mathrm{FeCl}_{3}$ & TFA & $\mathrm{DMF}$ & 80 & 5 \\
\hline 28 & $\mathrm{FeCl}_{3}$ & $\mathrm{PhCO}_{2} \mathrm{H}$ & DMF & 80 & 5 \\
\hline 29 & $\mathrm{FeCl}_{3}$ & (R)-(-)-BNP acid & DMF & 80 & 5 \\
\hline
\end{tabular}

[a] Reaction conditions: Reaction conditions: 1a $(1 \mathrm{mmol}), \mathbf{2 a}(1.2 \mathrm{mmol})$, catalyst (10 mol\%), solvent (1 mL), under open air. [b] Yields of isolated products. [c] With 5 mol\% $\mathrm{FeCl}_{3}$. [d] With $20 \mathrm{~mol} \% \mathrm{FeCl}_{3}$. [e] With $50 \mathrm{~mol} \% \mathrm{FeCl}_{3}$. [f] With $100 \mathrm{~mol} \%$ $\mathrm{FeCl}_{3}$. [g] With 5 mol\% p-TSA. [g] With 30 mol\% p-TSA. [g] With 50 mol\% p-TSA.

\subsection{Investigation of the reaction scope}

Scheme S1. The reaction of indolin-2-one with aliphatic aminoamides.<smiles>NC(=O)C1CCCN1</smiles>

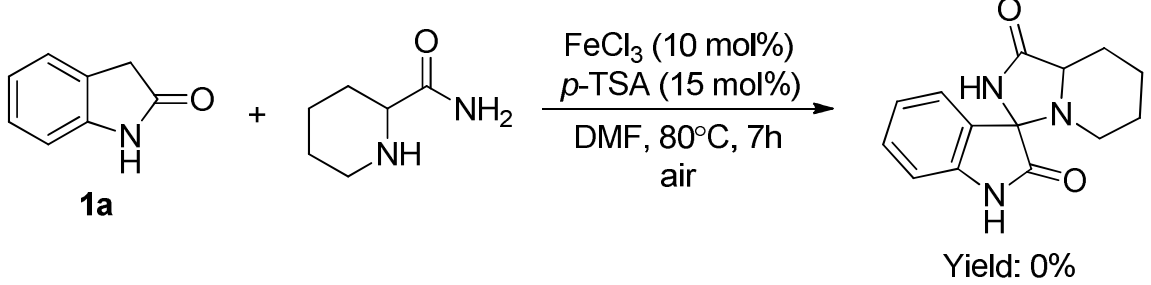

\section{Experimental procedure}

3.1 General procedure for the synthesis of spiro[indoline-3,2'-quinazolines] (3) from indolin-2-ones (1) and 2-aminobenzamides (2)

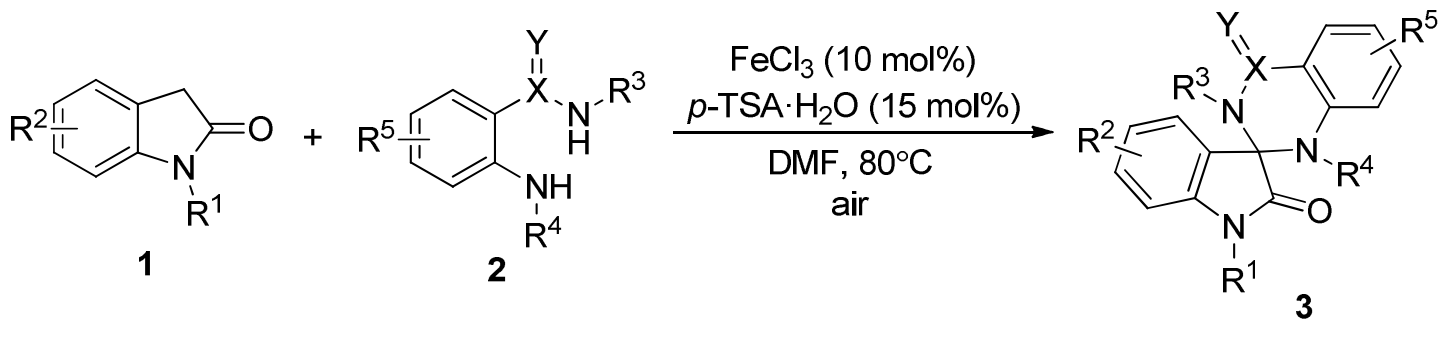


In a 10-mL reaction flask, equipped with a magnetic stirring bar, indolin-2-ones (1.0 mmol), 2-aminobenzamides (1.2 mmol), $p$-TSA $\cdot \mathrm{H}_{2} \mathrm{O}(15 \mathrm{~mol} \%, 28.5 \mathrm{mg})$ and $\mathrm{FeCl}_{3}$ $(10 \mathrm{~mol} \%, 16.2 \mathrm{mg})$ were added to DMF $(1.0 \mathrm{~mL})$. Then the resulting mixture was stirred at $80^{\circ} \mathrm{C}$ (oil bath) in the presence of ambient air. The formation of the products was monitored by TLC. After completion of the reaction, cold water $(20 \mathrm{~mL})$ was added. The mixture was extracted with ethyl acetate $(3 \times 20 \mathrm{~mL})$. The organic layer was concentrated under reduced pressure and the residue was purified by silica gel column chromatography using ethyl acetate /petroleum (1:5) as eluent to afford the pure product.

\subsection{Procedure for scale-up reaction}

In a 25-mL reaction flask, equipped with a magnetic stirring bar, indolin-2-one (5.0 mmol, 0.67g), 2-aminobenzamides (6.0 mmol, 0.82g), $p$-TSA $\mathrm{H}_{2} \mathrm{O}(15 \mathrm{~mol} \%, 0.14 \mathrm{~g})$ and $\mathrm{FeCl}_{3}(10 \mathrm{~mol} \%, 0.08 \mathrm{~g})$ were added to DMF $(5.0 \mathrm{~mL})$. Then the resulting mixture was stirred at $80{ }^{\circ} \mathrm{C}$ (oil bath) in the presence of ambient air. The formation of the $\mathbf{3 a}$ was monitored by TLC. After completion of the reaction, cold water $(80 \mathrm{~mL})$ was added. The mixture was extracted with ethyl acetate $(3 \times 80 \mathrm{~mL})$. The organic layer was concentrated under reduced pressure and the residue was purified by silica gel column chromatography using ethyl acetate /petroleum (1:5) as eluent to afford the product $3 \mathbf{a}$ in $78 \%$ yield $(1.03 \mathrm{~g})$.

\subsection{Procedure for the synthesis of spiro[benzo[d]imidazole-2,3'-indolin]-2'-one}

(5)

1)<smiles>O=C1Cc2ccccc2N1</smiles>

1a<smiles>Nc1ccccc1N</smiles>

4

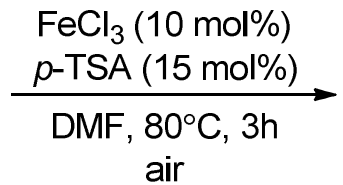

air

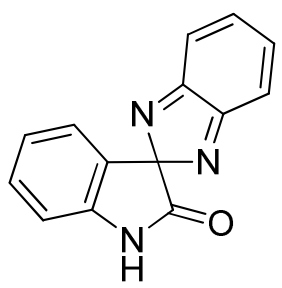

5, $85 \%$

In a $10-\mathrm{mL}$ reaction flask, equipped with a magnetic stirring bar, indolin-2-one (1.0 mmol, $133 \mathrm{mg}$ ), benzene-1,2-diamine (1.2 mmol, $130 \mathrm{mg}), p-\mathrm{TSA} \cdot \mathrm{H}_{2} \mathrm{O}(15 \mathrm{~mol} \%$, $28.5 \mathrm{mg})$ and $\mathrm{FeCl}_{3}(10 \mathrm{~mol} \%, 16.2 \mathrm{mg})$ were added to DMF $(1.0 \mathrm{~mL})$. Then the resulting mixture was stirred at $80^{\circ} \mathrm{C}$ (oil bath) for 3 hours in the presence of ambient air. After completion of the reaction, cold water $(20 \mathrm{~mL})$ was added. The mixture was 
extracted with ethyl acetate $(3 \times 20 \mathrm{~mL})$. The organic layer was concentrated under reduced pressure and the residue was purified by silica gel column chromatography using ethyl acetate/petroleum (1:5) as eluent to afford the pure product $\mathbf{1 3}$ in $85 \%$ yield (200 mg).

3.4 Procedure for the synthesis of 4,5,6,7-tetrahydrospiro[benzo[d] imidazole-2,3'-indolin]-2'-one (7)<smiles>NC1CCCCC1N</smiles>

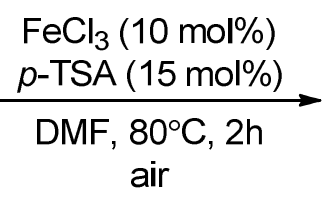

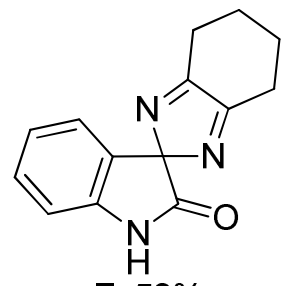

7, 53\%

In a $10-\mathrm{mL}$ reaction flask, equipped with a magnetic stirring bar, indolin-2-one (1.0 mmol, $133 \mathrm{mg})$, cyclohexane-1,2-diamine (1.2 mmol, $137 \mathrm{mg}), p$-TSA $\cdot \mathrm{H}_{2} \mathrm{O}(15 \mathrm{~mol} \%$, $28.5 \mathrm{mg})$ and $\mathrm{FeCl}_{3}(10 \mathrm{~mol} \%, 16.2 \mathrm{mg})$ were added to DMF $(1.0 \mathrm{~mL})$. Then the resulting mixture was stirred at $80^{\circ} \mathrm{C}$ (oil bath) for 2 hours in the presence of ambient air. After completion of the reaction, cold water $(20 \mathrm{~mL})$ was added. The mixture was extracted with ethyl acetate $(3 \times 20 \mathrm{~mL})$. The organic layer was concentrated under reduced pressure and the residue was purified by silica gel column chromatography using ethyl acetate /petroleum (1:5) as eluent to afford the pure product 7 in 53\% yield (127 mg).

3.5 Procedure for the synthesis of 5-chloro-1',3'-dihydrospiro[indoline-3,2'-perimidin]-2-one (9)

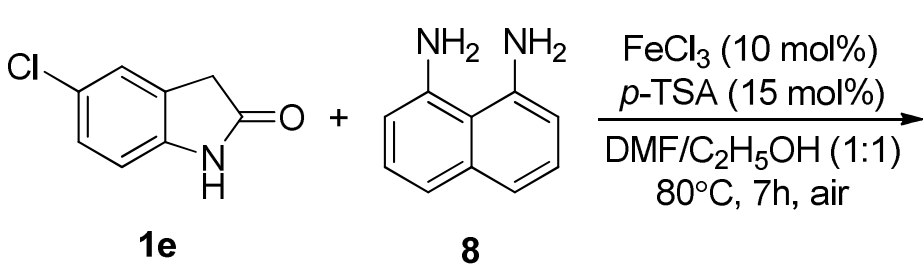

8<smiles>O=C1Nc2ccc(Cl)cc2C12Nc1cccc3cccc(c13)N2</smiles>

9, $37 \%$

In a $10-\mathrm{mL}$ reaction flask, equipped with a magnetic stirring bar, 5-chloroindolin-2-one ( $0.5 \mathrm{mmol}, 83.8 \mathrm{mg})$, naphthalene-1,8-diamine (0.6 mmol, 94.9 $\mathrm{mg}), p$-TSA $\cdot \mathrm{H}_{2} \mathrm{O}(15 \mathrm{~mol} \%, 14.3 \mathrm{mg})$ and $\mathrm{FeCl}_{3}(10 \mathrm{~mol} \%, 8.1 \mathrm{mg})$ were added to DMF $(0.6 \mathrm{~mL})$. Then the resulting mixture was stirred at $80{ }^{\circ} \mathrm{C}$ (oil bath) for 7 hours 
in the presence of ambient air. After completion of the reaction, cold water $(10 \mathrm{~mL})$ was added. The mixture was extracted with ethyl acetate $(3 \times 15 \mathrm{~mL})$. The organic layer was concentrated under reduced pressure and the residue was purified by silica gel column chromatography using ethyl acetate /petroleum (1:5) as eluent to afford the pure product 9 in $37 \%$ yield $(59.5 \mathrm{mg})$.

\subsection{Procedure for the synthesis of $3 \mathrm{H}$-spiro[benzo[d]thiazole-2,3'-indolin]-2'-one}<smiles>O=C1Cc2ccccc2N1</smiles>

$1 \mathrm{a}$<smiles>Nc1ccccc1S</smiles>

10

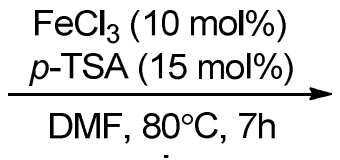

air

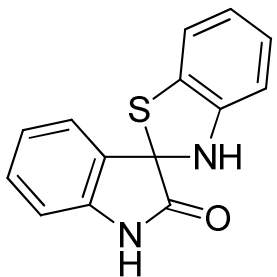

$11,16 \%$

In a $10-\mathrm{mL}$ reaction flask, equipped with a magnetic stirring bar, indolin-2-one (1.0 mmol, $133 \mathrm{mg}), 2$-aminobenzenethiol (1.2 mmol, $150 \mathrm{mg}), p-\mathrm{TSA} \cdot \mathrm{H}_{2} \mathrm{O}(15 \mathrm{~mol} \%$, $28.5 \mathrm{mg})$ and $\mathrm{FeCl}_{3}(10 \mathrm{~mol} \%, 16.2 \mathrm{mg})$ were added to DMF $(1.0 \mathrm{~mL})$. Then the resulting mixture was stirred at $80^{\circ} \mathrm{C}$ (oil bath) for 7 hours in the presence of ambient air. After completion of the reaction, cold water $(20 \mathrm{~mL})$ was added. The mixture was extracted with ethyl acetate $(3 \times 20 \mathrm{~mL})$. The organic layer was concentrated under reduced pressure and the residue was purified by silica gel column chromatography using ethyl acetate/petroleum (1:5) as eluent to afford the pure product $\mathbf{1 1}$ in $16 \%$ yield $(40.7 \mathrm{mg})$.

\subsection{Procedure for the synthesis of 5-chloro-2',3', $4^{\prime}, 9^{\prime}-$}

\section{tetrahydrospiro[indoline-3,1'-pyrido[3,4-b]indol]-2-one (13)}<smiles>NCCc1c[nH]c2c1=C[C+]=CC=2</smiles>

12

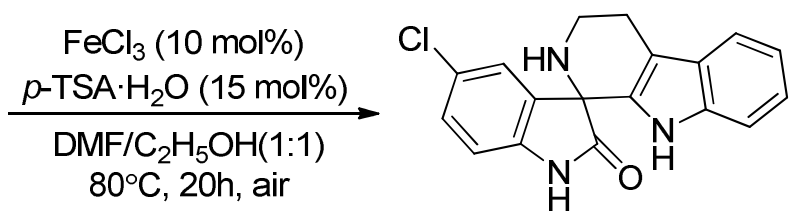

$13,55 \%$

In a $10-\mathrm{mL}$ reaction flask, equipped with a magnetic stirring bar, 5-chloroindolin-2-one (1.0 mmol, $168 \mathrm{mg})$, tryptamine (1.2 mmol, $192 \mathrm{mg})$, $p$-TSA $\cdot \mathrm{H}_{2} \mathrm{O}(15 \mathrm{~mol} \%, 28.5 \mathrm{mg})$ and $\mathrm{FeCl}_{3}(10 \mathrm{~mol} \%, 16.2 \mathrm{mg})$ were added to a mixed solvent of DMF and ethanol $(1: 1,1.0 \mathrm{~mL})$. Then the resulting mixture was 
stirred at $80^{\circ} \mathrm{C}$ (oil bath) for 20 hours in the presence of ambient air. After completion of the reaction, cold water $(20 \mathrm{~mL})$ was added. The mixture was extracted with ethyl acetate $(3 \times 20 \mathrm{~mL})$. The organic layer was concentrated under reduced pressure and the residue was purified by silica gel column chromatography using ethyl acetate /petroleum (1:6) as eluent to afford the pure product 13 in 55\% yield (178 mg).

\subsection{Procedure for control experiment (Scheme 5d, isatin(16))}

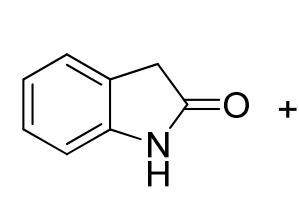

$1 \mathrm{a}$<smiles>NC(=O)c1ccccc1</smiles>

14

$$
\begin{aligned}
& \underset{\mathrm{FeCl}_{3}(10 \mathrm{~mol} \%)}{p-\mathrm{TSA}(15 \mathrm{~mol} \%)} \\
& \underset{\mathrm{DMF}(1 \mathrm{M}), 80^{\circ} \mathrm{C}}{\longrightarrow}
\end{aligned}
$$

air<smiles>O=C1Nc2ccccc2C1=NC(=O)c1ccccc1</smiles>

$15,0 \%$

16, $37 \%$

In a 10-mL reaction flask, equipped with a magnetic stirring bar, indolin-2-one (1.0 mmol, $133 \mathrm{mg})$, benzamide (1.2 mmol, $145 \mathrm{mg}), p-\mathrm{TSA} \cdot \mathrm{H}_{2} \mathrm{O}(15 \mathrm{~mol} \%, 28.5 \mathrm{mg})$ and $\mathrm{FeCl}_{3}(10 \mathrm{~mol} \%, 16.2 \mathrm{mg})$ were added to DMF $(1.0 \mathrm{~mL})$. Then the resulting mixture was stirred at $80{ }^{\circ} \mathrm{C}$ (oil bath) for 5 hours in the presence of ambient air. After completion of the reaction, cold water $(20 \mathrm{~mL})$ was added. The mixture was extracted with ethyl acetate $(3 \times 20 \mathrm{~mL})$. The organic layer was concentrated under reduced pressure and the residue was purified by silica gel column chromatography using ethyl acetate /petroleum (1:5) as eluent to afford the product $\mathbf{1 6}$ in 37\% yield (54.4 $\mathrm{mg})$.

\subsection{Procedure for control experiment (Scheme 5e,}

\section{3-((4-Bromophenyl)imino)indolin-2-one (18))}<smiles>O=C1Cc2ccccc2N1</smiles>

$1 \mathbf{a}$<smiles>Nc1ccc(Br)cc1</smiles>

17
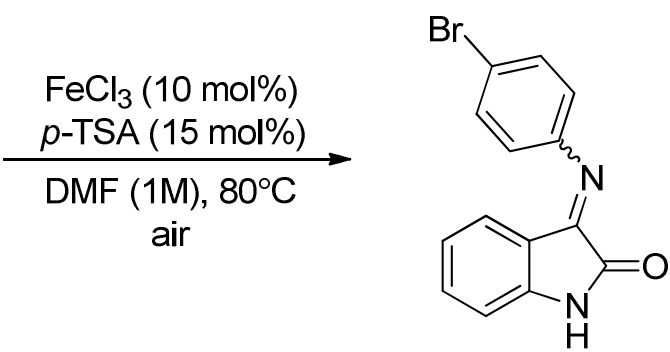

$18,41 \%(E: Z=78: 22)$

In a $10-\mathrm{mL}$ reaction flask, equipped with a magnetic stirring bar, indolin-2-one (1.0 mmol, $133 \mathrm{mg})$, 4-bromoaniline (1.2 mmol, $206 \mathrm{mg}), p$-TSA $\cdot \mathrm{H}_{2} \mathrm{O}(15 \mathrm{~mol} \%, 28.5 \mathrm{mg})$ and $\mathrm{FeCl}_{3}(10 \mathrm{~mol} \%, 16.2 \mathrm{mg})$ were added to DMF $(1.0 \mathrm{~mL})$. Then the resulting 
mixture was stirred at $80^{\circ} \mathrm{C}$ (oil bath) for 5 hours in the presence of ambient air. After completion of the reaction, cold water $(20 \mathrm{~mL})$ was added. The mixture was extracted with ethyl acetate $(3 \times 20 \mathrm{~mL})$. The organic layer was concentrated under reduced pressure and the residue was purified by silica gel column chromatography using ethyl acetate /petroleum (1:5) as eluent to afford the product $\mathbf{1 8}$ in 41\% yield (123 $\mathrm{mg})$.

\section{Characterization data of compounds 3a-3al}<smiles>O=C1NC2(Nc3ccccc31)C(=O)Nc1ccccc12</smiles>

\section{1'H-spiro[indoline-3,2'-quinazoline]-2,4'(3'H)-dione (3a) ${ }^{[1]}$}

Yield: $83 \%$ (220 mg). White solid, mp 267-268 ${ }^{\circ} \mathrm{C} .{ }^{1} \mathrm{H}$ NMR (400MHz, DMSO- $\left.d_{6}\right): \delta$ $6.61(\mathrm{~d}, 1 \mathrm{H}, \mathrm{ArH}, J=8.0 \mathrm{~Hz}), 6.68(\mathrm{t}, 1 \mathrm{H}, \mathrm{ArH}, J=7.6 \mathrm{~Hz}), 6.84(\mathrm{~d}, 1 \mathrm{H}, \mathrm{ArH}, J=$ $7.6 \mathrm{~Hz}), 7.05$ (t, 1H, ArH, $J=7.2 \mathrm{~Hz}), 7.22$ (t, 1H, ArH, $J=7.6 \mathrm{~Hz}), 7.26$ (s, 1H, NH), 7.32 (t, 1H, ArH, $J=7.6 \mathrm{~Hz}), 7.47$ (d, 1H, ArH, $J=7.2 \mathrm{~Hz}), 7.60$ (d, 1H, ArH, $J=$ 7.6Hz), 8.33 (s, $1 \mathrm{H}, \mathrm{NH}), 10.23(\mathrm{~s}, 1 \mathrm{H}, \mathrm{NH}) .{ }^{13} \mathrm{C}$ NMR (100MHz, DMSO- $\left.d_{6}\right): \delta 70.9$, $110.0,113.8,114.3,117.1,122.2,125.3,126.8,129.4,130.7,133.2,142.1,146.8$, $163.9,176.0$.<smiles>Cc1ccc2c(c1)C1(NC(=O)c3ccccc3N1)NC2=O</smiles>

\section{5-Methyl-1'H-spiro[indoline-3,2'-quinazoline]-2,4'(3'H)-dione (3b) ${ }^{[1]}$}

Yield: $56 \%(156 \mathrm{mg})$. White solid, $\mathrm{mp}>300^{\circ} \mathrm{C} .{ }^{1} \mathrm{H}$ NMR $\left(400 \mathrm{MHz}, \mathrm{DMSO}-d_{6}\right): \delta$ $2.27\left(\mathrm{~s}, 3 \mathrm{H}, \mathrm{CH}_{3}\right), 6.60$ (d, 1H, ArH, $\left.J=8.0 \mathrm{~Hz}\right), 6.67$ (t, 1H, ArH, $\left.J=7.6 \mathrm{~Hz}\right), 6.73$ (d, $1 \mathrm{H}, \mathrm{ArH}, J=8.0 \mathrm{~Hz}), 7.12(\mathrm{~d}, 1 \mathrm{H}, \mathrm{ArH}, J=7.6 \mathrm{~Hz}), 7.19-7.23$ (t, 2H, ArH, NH), 7.30 (s, 1H, ArH), 7.59 (d, 1H, ArH, $J=7.6 \mathrm{~Hz}), 8.30(\mathrm{~s}, 1 \mathrm{H}, \mathrm{NH}), 10.18(\mathrm{~s}, 1 \mathrm{H}, \mathrm{NH}) .{ }^{13} \mathrm{C}$ 
NMR (100MHz, DMSO- $\left.d_{6}\right): \delta 20.5,71.0,109.8,113.7,114.2,117.0,125.9,126.8$, $129.4,130.9,131.2,133.2,139.6,146.8,163.8,176.0$.<smiles>COc1ccc2c(c1)C1(NC(=O)c3ccccc3N1)NC2=O</smiles>

\section{5-Methoxy-1'H-spiro[indoline-3,2'-quinazoline]-2,4' (3'H)-dione (3c) $)^{[1]}$}

Yield: $63 \%(186 \mathrm{mg})$. Yellow solid, $\mathrm{mp}>300^{\circ} \mathrm{C} .{ }^{1} \mathrm{H}$ NMR $\left(400 \mathrm{MHz}, \mathrm{DMSO}-d_{6}\right): \delta$ $3.73\left(\mathrm{~s}, 3 \mathrm{H}, \mathrm{CH}_{3}\right), 6.61$ (d, 1H, ArH, $\left.J=8.0 \mathrm{~Hz}\right), 6.67$ (t, 1H, ArH, $\left.J=7.6 \mathrm{~Hz}\right), 6.77$ (d, 1H, ArH, $J=8.4 \mathrm{~Hz}), 6.90$ (d, 1H, ArH, $J=8.4 \mathrm{~Hz}), 7.09$ (s, 1H, ArH), 7.22 (t, 1H, $\operatorname{ArH}, J=7.6 \mathrm{~Hz}), 7.27$ (s, 1H, NH), 7.59 (d, 1H, ArH, $J=7.2 \mathrm{~Hz}), 8.33$ (s, 1H, NH), $10.11(\mathrm{~s}, 1 \mathrm{H}, \mathrm{NH}) .{ }^{13} \mathrm{C} \mathrm{NMR}\left(100 \mathrm{MHz}, \mathrm{DMSO}-d_{6}\right): \delta 55.6,71.3,110.6,111.6,113.8$, $114.3,115.9,117.1,126.8,130.4,133.2,135.2,146.8,155.3,163.8$.<smiles>O=C1NC2(Nc3ccccc31)C(=O)Nc1ccc(F)cc12</smiles>

\section{5-Fluoro-1'H-spiro[indoline-3,2'-quinazoline]-2,4'(3'H)-dione (3d) ${ }^{[1]}$}

Yield: $81 \%$ (229 mg). White solid, mp 259-260 ${ }^{\circ} \mathrm{C} .{ }^{1} \mathrm{H}$ NMR $\left(400 \mathrm{MHz}, \mathrm{DMSO}-d_{6}\right): \delta$ $6.62(\mathrm{~d}, 1 \mathrm{H}, \mathrm{ArH}, J=8.0 \mathrm{~Hz}), 6.70$ (t, $1 \mathrm{H}, \mathrm{ArH}, J=7.2 \mathrm{~Hz}), 6.84-6.87$ (dd, 1H, ArH, $\left.J_{l}=8.4 \mathrm{~Hz}, J_{2}=4.0 \mathrm{~Hz}\right), 7.19\left(\mathrm{dt}, 1 \mathrm{H}, \mathrm{ArH}, J_{1}=9.2 \mathrm{~Hz}, J_{2}=2.8 \mathrm{~Hz}\right), 7.24(\mathrm{t}, 1 \mathrm{H}, \mathrm{ArH}$, $J=7.6 \mathrm{~Hz}), 7.33(\mathrm{~s}, 1 \mathrm{H}, \mathrm{NH}), 7.38\left(\mathrm{dd}, 1 \mathrm{H}, \mathrm{ArH}, J_{1}=8.0 \mathrm{~Hz}, J_{2}=2.4 \mathrm{~Hz}\right), 7.61(\mathrm{~d}, 1 \mathrm{H}$, $\mathrm{ArH}, J=7.6 \mathrm{~Hz}), 8.40(\mathrm{~s}, 1 \mathrm{H}, \mathrm{NH}), 10.33(\mathrm{~s}, 1 \mathrm{H}, \mathrm{NH}) .{ }^{13} \mathrm{C} \mathrm{NMR}(100 \mathrm{MHz}$, $\left.\mathrm{CDCl}_{3}+\mathrm{DMSO}-d_{6}\right): \delta 71.2,110.9,112.9,113.2,113.9,114.2,117.3,126.8,131.0$, $133.3,138.3,146.6,158.2\left(\mathrm{~d},{ }^{1} J_{C F}=236 \mathrm{~Hz}, \mathrm{C}-\mathrm{F}\right), 163.7,176.1$.<smiles>O=C1NC2(Nc3ccccc31)C(=O)Nc1ccc(Cl)cc12</smiles> 


\section{5-Chloro-1'H-spiro[indoline-3,2'-quinazoline]-2,4' (3'H)-dione (3e) ${ }^{[1]}$}

Yield: 93\% (279 mg). Pale yellow solid, mp 275-278 ${ }^{\circ} \mathrm{C} .{ }^{1} \mathrm{H}$ NMR (400MHz, DMSO- $\left.d_{6}\right): \delta 6.60(\mathrm{~d}, 1 \mathrm{H}, \mathrm{ArH}, J=8.0 \mathrm{~Hz}), 6.70(\mathrm{t}, 1 \mathrm{H}, \mathrm{ArH}, J=7.6 \mathrm{~Hz}), 6.86(\mathrm{~d}, 1 \mathrm{H}$, $\operatorname{ArH}, J=8.0 \mathrm{~Hz}), 7.24$ (t, 1H, ArH, $J=7.6 \mathrm{~Hz}), 7.33$ (s, 1H, NH), 7.39 (d, 1H, ArH, $J$ $=8.0 \mathrm{~Hz}), 7.50(\mathrm{~s}, 1 \mathrm{H}, \mathrm{ArH}), 7.60(\mathrm{~d}, 1 \mathrm{H}, \mathrm{ArH}, J=7.6 \mathrm{~Hz}), 8.39(\mathrm{~s}, 1 \mathrm{H}, \mathrm{NH}), 10.43(\mathrm{~s}$, $1 \mathrm{H}, \mathrm{NH}) .{ }^{13} \mathrm{C}$ NMR $\left(100 \mathrm{MHz}, \mathrm{DMSO}-d_{6}\right): \delta 71.0,111.6,113.9,114.2,117.4,125.4$, $126.1,126.9,130.6,131.3,133.4,141.0,146.5,163.7,175.8$.<smiles>O=C1NC2(Nc3ccccc31)C(=O)Nc1ccc(Br)cc12</smiles>

\section{5-Bromo-1'H-spiro[indoline-3,2'-quinazoline]-2,4' $\left(3^{\prime} \mathbf{H}\right)$-dione $(3 \mathrm{f})^{[1]}$}

Yield: $84 \%$ (289 mg). White solid, mp 283-285 ${ }^{\circ} \mathrm{C} .{ }^{1} \mathrm{H}$ NMR (400MHz, DMSO- $\left.d_{6}\right): \delta$ $6.61(\mathrm{~d}, 1 \mathrm{H}, \operatorname{ArH}, J=8.0 \mathrm{~Hz}), 6.71(\mathrm{t}, 1 \mathrm{H}, \mathrm{ArH}, J=7.2 \mathrm{~Hz}), 6.83$ (d, 1H, ArH, $J=$ $8.4 \mathrm{~Hz}), 7.25$ (t, 1H, $\mathrm{ArH}, J=7.6 \mathrm{~Hz}), 7.34$ (s, $1 \mathrm{H}, \mathrm{NH}), 7.53$ (d, 1H, $\mathrm{ArH}, J=8.4 \mathrm{~Hz}$ ), $7.60(\mathrm{~s}, 1 \mathrm{H}, \mathrm{ArH}), 7.61(\mathrm{~d}, 1 \mathrm{H}, \mathrm{ArH}, J=6.8 \mathrm{~Hz}), 8.40(\mathrm{~s}, 1 \mathrm{H}, \mathrm{NH}), 10.45$ (s, 1H, NH). ${ }^{13} \mathrm{C}$ NMR $\left(100 \mathrm{MHz}, \mathrm{DMSO}-d_{6}\right): \delta 71.0,112.1,113.7,113.9,114.2,117.4,126.9$, $128.1,131.7,133.4,133.4,141.4,146.5,163.7,175.6$.<smiles>O=C1NC2(Nc3ccccc31)C(=O)Nc1ccc([N+](=O)[O-])cc12</smiles>

\section{5-Nitro-1'H-spiro[indoline-3,2'-quinazoline]-2,4'(3'H)-dione (3g) ${ }^{[2]}$}

Yield: 90\% (279 mg). Light yellow solid, mp $313{ }^{\circ} \mathrm{C} .{ }^{1} \mathrm{H}$ NMR (400MHz, DMSO- $\left.d_{6}\right)$ : $\delta 6.63(\mathrm{~d}, 1 \mathrm{H}, \operatorname{ArH}, J=8.0 \mathrm{~Hz}), 6.75$ (t, 1H, ArH, $J=15.2 \mathrm{~Hz}), 7.08$ (d, 1H, ArH, $J=$ $8.4 \mathrm{~Hz}), 7.26-7.30(\mathrm{~m}, 1 \mathrm{H}, \mathrm{ArH}), 7.41(\mathrm{~s}, 1 \mathrm{H}, \mathrm{NH}), 7.64\left(\mathrm{dd}, 1 \mathrm{H}, \mathrm{ArH}, J_{l}=7.6 \mathrm{~Hz}, J_{2}\right.$ $=1.2 \mathrm{~Hz}), 8.24(\mathrm{~d}, 1 \mathrm{H}, \operatorname{ArH}, J=2.4 \mathrm{~Hz}), 8.31\left(\mathrm{dd}, 1 \mathrm{H}, \operatorname{ArH}, J_{l}=2.4 \mathrm{~Hz}, J_{2}=8.4 \mathrm{~Hz}\right)$, $8.45(\mathrm{~s}, 1 \mathrm{H}, \mathrm{NH}), 11.04(\mathrm{~s}, 1 \mathrm{H}, \mathrm{NH}) .{ }^{13} \mathrm{C} \mathrm{NMR}\left(100 \mathrm{MHz}, \mathrm{DMSO}-d_{6}\right): \delta 70.7,110.5$, $114.1,114.2,117.8,120.7,126.9,127.8,130.4,133.6,142.5,146.2,148.6,163.5$, 176.3 . 
<smiles>O=C1NC2(Nc3ccccc31)C(=O)Nc1ccccc12</smiles>

\section{4-Chloro-1'H-spiro[indoline-3,2'-quinazoline]-2,4'(3'H)-dione (3h)}

Yield: $80 \%$ (240 mg). Light orange solid, mp 292-293 ${ }^{\circ} \mathrm{C} .{ }^{1} \mathrm{H}$ NMR $(400 \mathrm{MHz}$, DMSO- $\left.d_{6}\right): \delta 6.56(\mathrm{~d}, 1 \mathrm{H}, \mathrm{ArH}, J=8.0 \mathrm{~Hz}), 6.64(\mathrm{t}, 1 \mathrm{H}, \mathrm{ArH}, J=7.2 \mathrm{~Hz}), 6.81(\mathrm{~d}, 1 \mathrm{H}$, $\operatorname{ArH}, J=8.0 \mathrm{~Hz}), 7.04$ (d, 1H, ArH, $J=8.0 \mathrm{~Hz}), 7.21$ (t, $1 \mathrm{H}, \mathrm{ArH}, J=7.6 \mathrm{~Hz}), 7.32$ (s, $1 \mathrm{H}, \mathrm{NH}), 7.32$ (t, 1H, ArH, $J=7.6 \mathrm{~Hz}), 7.59$ (d, 1H, ArH, $J=7.6 \mathrm{~Hz}), 8.40$ (s, 1H, $\mathrm{NH}), 10.53$ (s, 1H, NH). ${ }^{13} \mathrm{C}$ NMR (100MHz, DMSO-d $): \delta 71.9,109.1,113.1,113.4$, 116.6, 123.1, 125.6, 126.8, 131.6, 132.2, 133.3, 144.0, 146.6, 163.1, 175.1. HRMS (ESI) $\mathrm{m} / \mathrm{z}$ calcd for $\mathrm{C}_{15} \mathrm{H}_{11} \mathrm{ClN}_{3} \mathrm{O}_{2}\left([\mathrm{M}+\mathrm{H}]^{+}\right)$: 300.0534; found: 300.0537 .<smiles>O=C1NC2(Nc3ccccc31)C(=O)Nc1cc(Cl)ccc12</smiles>

\section{6-Chloro-1'H-spiro[indoline-3,2'-quinazoline]-2,4'(3'H)-dione (3i)}

Yield: 49\% (147 mg). Light yellow solid, mp 289-290 ${ }^{\circ} \mathrm{C} .{ }^{1} \mathrm{H}$ NMR (400MHz, DMSO-d $)): \delta 6.60(\mathrm{~d}, 1 \mathrm{H}, \mathrm{ArH}, J=8.0 \mathrm{~Hz}), 6.70(\mathrm{t}, 1 \mathrm{H}, \mathrm{ArH}, J=7.6 \mathrm{~Hz}), 6.89(\mathrm{~d}, 1 \mathrm{H}$, $\operatorname{ArH}, J=2.0 \mathrm{~Hz}), 7.09\left(\mathrm{dd}, 1 \mathrm{H}, \mathrm{ArH}, J_{1}=7.6 \mathrm{~Hz}, J_{2}=1.6 \mathrm{~Hz}\right), 7.21-7.26(\mathrm{~m}, 1 \mathrm{H}, \mathrm{ArH})$, 7.28-7.48 (d, $1 \mathrm{H}, \mathrm{ArH}, J=8.0 \mathrm{~Hz}), 7.60\left(\mathrm{dd}, 1 \mathrm{H}, \mathrm{ArH}, J_{1}=6.4 \mathrm{~Hz}, J_{2}=1.6 \mathrm{~Hz}\right), 8.34(\mathrm{~s}$, $1 \mathrm{H}, \mathrm{NH}), 10.45(\mathrm{~s}, 1 \mathrm{H}, \mathrm{NH}) .{ }^{13} \mathrm{C} \mathrm{NMR}\left(100 \mathrm{MHz}, \mathrm{DMSO}-d_{6}\right): \delta 70.6,110.1,113.9$, $114.2,117.3,122.0,126.8,128.3,133.4,134.9,143.7,146.6,163.7,175.9$. HRMS (ESI) $\mathrm{m} / \mathrm{z}$ calcd for $\mathrm{C}_{15} \mathrm{H}_{11} \mathrm{ClN}_{3} \mathrm{O}_{2}\left([\mathrm{M}+\mathrm{H}]^{+}\right)$: 300.0534; found: 300.0535 .<smiles>O=C1NC2(Nc3ccccc31)C(=O)Nc1c(F)cccc12</smiles>

\section{7-Fluoro-1'H-spiro[indoline-3,2'-quinazoline]-2,4'(3'H)-dione (3j)}


Yield: 84\% (238 mg). Light yellow solid, mp 284-285 ${ }^{\circ} \mathrm{C} .{ }^{1} \mathrm{H}$ NMR (400MHz, DMSO- $\left.d_{6}\right): \delta 6.62(\mathrm{~d}, 1 \mathrm{H} \operatorname{ArH}, J=8.0 \mathrm{~Hz}),, 6.69-6.73(\mathrm{~m}, 1 \mathrm{H}, \operatorname{ArH}), 7.06-7.11(\mathrm{~m}$, $1 \mathrm{H}, \operatorname{ArH}), 7.23-7.30$ (m, 2H, ArH), 7.33 (s, 1H, NH), 7.34(d, 1H, ArH, $J=4.4 \mathrm{~Hz}$ ), $7.62\left(\mathrm{dd}, 1 \mathrm{H}, \mathrm{ArH}, J_{1}=6.4 \mathrm{~Hz}, J_{2}=1.2 \mathrm{~Hz}\right), 8.38(\mathrm{~s}, 1 \mathrm{H}, \mathrm{NH}), 10.84(\mathrm{~s}, 1 \mathrm{H}, \mathrm{NH}) .{ }^{13} \mathrm{C}$ NMR (100MHz, DMSO-d $\left.d_{6}\right): \delta 71.1,113.9,114.1,117.4,121.3,123.2,126.9,129.2$, 132.3, 133.4, 146.3 (d, $\left.{ }^{1} J_{C F}=241 \mathrm{~Hz}, \mathrm{C}-\mathrm{F}\right), 146.5,163.7,175.8,277.0$. HRMS (ESI) $\mathrm{m} / \mathrm{z}$ calcd for $\mathrm{C}_{15} \mathrm{H}_{11} \mathrm{FN}_{3} \mathrm{O}_{2}\left([\mathrm{M}+\mathrm{H}]^{+}\right)$: 284.0830; found: 284.0833 .

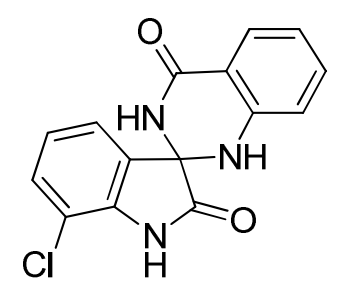

\section{7-Chloro-1'H-spiro[indoline-3,2'-quinazoline]-2,4'(3'H)-dione (3k)}

Yield: 72\% (216 mg). Light brown solid, mp 305-306 ${ }^{\circ} \mathrm{C} .{ }^{1} \mathrm{H}$ NMR $(400 \mathrm{MHz}$, DMSO- $\left.d_{6}\right): \delta 6.60(\mathrm{~d}, 1 \mathrm{H}, \operatorname{ArH}, J=8.0 \mathrm{~Hz}), 6.70(\mathrm{t}, 1 \mathrm{H}, \operatorname{ArH}, J=7.6 \mathrm{~Hz}), 7.08$ (t, $1 \mathrm{H}, \mathrm{ArH}, J=7.6 \mathrm{~Hz}), 7.24$ (t, 1H, ArH, $J=7.2 \mathrm{~Hz}), 7.34$ (s, 1H, NH), 7.40 (d, 1H, $\operatorname{ArH}, J=8.0 \mathrm{~Hz}), 7.44(\mathrm{~d}, 1 \mathrm{H}, \mathrm{ArH}, J=7.2 \mathrm{~Hz}), 7.61$ (d, $1 \mathrm{H}, \operatorname{ArH}), J=7.2 \mathrm{~Hz}$, $8.38(\mathrm{~s}, 1 \mathrm{H}, \mathrm{NH}), 10.76(\mathrm{~s}, 1 \mathrm{H}, \mathrm{NH}) .{ }^{13} \mathrm{C} \mathrm{NMR}\left(400 \mathrm{MHz}, \mathrm{DMSO}-d_{6}\right): \delta 71.6,113.9$, 114.2 , 114.2, 117.4, 123.6, 126.9, 130.6, 131.4, 133.4, 139.8, 146.5, 163.7, 176.0 . HRMS (ESI) m/z calcd for $\mathrm{C}_{15} \mathrm{H}_{11} \mathrm{ClN}_{3} \mathrm{O}_{2}\left([\mathrm{M}+\mathrm{H}]^{+}\right)$: 300.0534; found: 300.0535 .

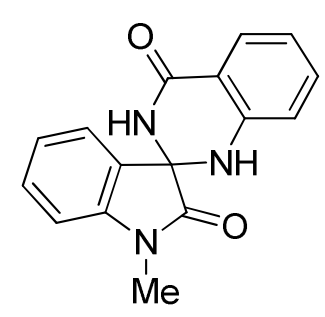

\section{1-Methyl-1'H-spiro[indoline-3,2'-quinazoline]-2,4'(3'H)-dione $(3 \mathrm{l})^{[3]}$}

Yield: 83\% (232 mg). White solid, mp 272-273 ${ }^{\circ} \mathrm{C} .{ }^{1} \mathrm{H}$ NMR (400MHz, DMSO- $\left.d_{6}\right): \delta$ $3.08\left(\mathrm{~s}, 3 \mathrm{H}, \mathrm{CH}_{3}\right), 6.61(\mathrm{~d}, 1 \mathrm{H}, \mathrm{ArH}, J=8.0 \mathrm{~Hz}), 6.71(\mathrm{t}, 1 \mathrm{H}, \mathrm{ArH}, J=7.2 \mathrm{~Hz}), 7.08(\mathrm{~d}$, $1 \mathrm{H}, \operatorname{ArH}, J=8.0 \mathrm{~Hz}), 7.15$ (t, 1H, ArH, $J=7.6 \mathrm{~Hz}), 7.25$ (t, 1H, ArH, $J=8.0 \mathrm{~Hz}$ ), 7.26 $(\mathrm{s}, 1 \mathrm{H}, \mathrm{NH}), 7.45$ (t, 1H, ArH, $J=8.0 \mathrm{~Hz}), 7.53$ (d, 1H, ArH, $J=7.2 \mathrm{~Hz}), 7.63$ (d, 1H, $\mathrm{ArH}, J=7.6 \mathrm{~Hz}), 8.30(\mathrm{~s}, 1 \mathrm{H}, \mathrm{NH}) .{ }^{13} \mathrm{C} \mathrm{NMR}\left(100 \mathrm{MHz}, \mathrm{DMSO}-d_{6}\right): \delta 25.9,70.8$, 
$108.9,113.9,114.2,117.3,122.9,124.9,126.9,128.8,130.9,133.3,143.5,146.7$, 163.8, 174.2.

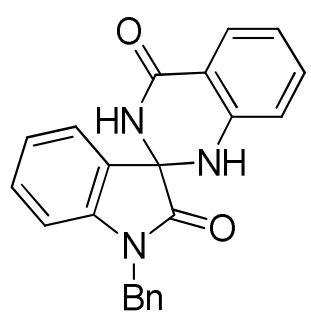

\section{1-Benzyl-1'H-spiro[indoline-3,2'-quinazoline]-2,4'(3'H)-dione $(3 \mathrm{~m})^{[3]}$}

Yield: 87\% (309 mg). White solid, mp 274-275 ${ }^{\circ} \mathrm{C} .{ }^{1} \mathrm{H}$ NMR (400MHz, DMSO- $\left.d_{6}\right): \delta$ $4.84\left(\mathrm{~s}, 2 \mathrm{H}, \mathrm{CH}_{2}\right), 6.67$ (d, 1H, ArH, $\left.J=8.0 \mathrm{~Hz}\right), 6.73$ (t, 1H, ArH, $\left.J=7.6 \mathrm{~Hz}\right), 6.91$ (d, $1 \mathrm{H}, \operatorname{ArH}, J=8.0 \mathrm{~Hz}), 7.14$ (t, $1 \mathrm{H}, \operatorname{ArH}, J=7.2 \mathrm{~Hz}), 7.24-7.31$ (m, 2H, ArH), 7.34 (s, 1H, NH), 7.36-7.39 (m, 3H, ArH), 7.42 (d, 2H, ArH, J=5.2Hz), 7.59 (d, 1H, ArH, $J$ $=7.2 \mathrm{~Hz}), 7.65(\mathrm{~d}, 1 \mathrm{H}, \operatorname{ArH}, J=7.6 \mathrm{~Hz}), 8.52(\mathrm{~s}, 1 \mathrm{H}, \mathrm{NH}) .{ }^{13} \mathrm{C} \mathrm{NMR}(100 \mathrm{MHz}$, DMSO- $\left.d_{6}\right): \delta 42.6,70.8,109.6,113.9,114.3,117.3,123.0,125.2,126.9,127.3,127.4$, $128.5,128.8,130.8,133.3,135.7,142.7,146.7,163.9,174.5$.

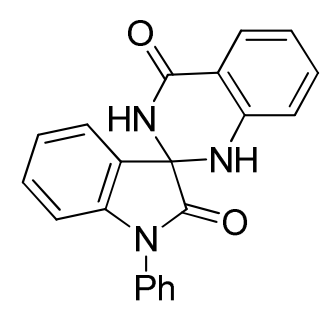

1-Phenyl-1'H-spiro[indoline-3,2'-quinazoline]-2,4'(3'H)-dione (3n) $)^{[4]}$

Yield: $91 \%(311 \mathrm{mg})$. White solid, mp 249-251 ${ }^{\circ} \mathrm{C} .{ }^{1} \mathrm{H}$ NMR (400MHz, DMSO- $\left.d_{6}\right): \delta$ $6.66(\mathrm{~d}, 1 \mathrm{H}, \mathrm{ArH}, J=8.0 \mathrm{~Hz}), 6.71(\mathrm{t}, 1 \mathrm{H}, \mathrm{ArH}, J=7.2 \mathrm{~Hz}), 6.78$ (d, 1H, ArH, $J=$ $8.0 \mathrm{~Hz}), 7.20(\mathrm{t}, 1 \mathrm{H}, \mathrm{ArH}, J=7.6 \mathrm{~Hz}), 7.26(\mathrm{t}, 1 \mathrm{H}, \mathrm{ArH}, J=8.0 \mathrm{~Hz}), 7.36-7.40(\mathrm{~m}, 2 \mathrm{H}$, $\operatorname{ArH}), 7.41$ (s, 1H, NH), 7.47 (t, 2H, ArH, $J=8.0 H z), 7.57-7.64$ (m, 4H, ArH), 8.45 (s, $1 \mathrm{H}, \mathrm{NH}) .{ }^{13} \mathrm{C}$ NMR $\left(100 \mathrm{MHz}, \mathrm{DMSO}-d_{6}\right): \delta 71.1,109.3,114.0,114.1,117.5,123.5$, $125.5,126.5,126.9,128.2,128.8,129.7,130.9,133.4,133.8,143.3,146.6,163.8$, 173.6. 
<smiles>CN1C(=O)C2(NC(=O)c3ccccc32)Nc2ccc(F)cc21</smiles>

\section{5-Fluoro-1-methyl-1'H-spiro[indoline-3,2'-quinazoline]-2,4'(3'H)-dione (3o)}

Yield: $82 \%$ (244 mg). White solid, mp $283-285^{\circ} \mathrm{C} .{ }^{1} \mathrm{H}$ NMR (400MHz, DMSO- $\left.d_{6}\right): \delta$ $3.10\left(\mathrm{~s}, 3 \mathrm{H}, \mathrm{CH}_{3}\right), 6.63(\mathrm{~d}, 1 \mathrm{H}, \mathrm{ArH}, J=8.0 \mathrm{~Hz}), 6.73(\mathrm{t}, 1 \mathrm{H}, \mathrm{ArH}, J=7.6 \mathrm{~Hz}), 7.10$ $\left(\mathrm{dd}, 1 \mathrm{H}, \mathrm{ArH}, J_{1}=4.0 \mathrm{~Hz}, J_{2}=8.4 \mathrm{~Hz}\right), 7.25-7.34(\mathrm{~m}, 3 \mathrm{H}, \mathrm{ArH}, \mathrm{NH}), 7.45$ (dd, 1H, ArH, $\left.J_{1}=2.4 \mathrm{~Hz}, J_{2}=7.6 \mathrm{~Hz}\right), 7.64(\mathrm{~d}, 1 \mathrm{H}, \mathrm{ArH}, J=7.6 \mathrm{~Hz}), 8.36(\mathrm{~s}, 1 \mathrm{H}, \mathrm{NH}) .{ }^{13} \mathrm{C} \mathrm{NMR}$ $\left(100 \mathrm{MHz}, \mathrm{DMSO}-d_{6}\right): \delta 26.0,71.0,109.9,112.8,113.9,114.2,117.2,117.5,126.9$, $130.5,133.4,139.8,146.5,158.8\left(\mathrm{~d},{ }^{1} J_{C F}=238 \mathrm{~Hz}, \mathrm{C}-\mathrm{F}\right), 163.7,174.2$. HRMS (ESI) m/z calcd for $\mathrm{C}_{16} \mathrm{H}_{13} \mathrm{FN}_{3} \mathrm{O}_{2}\left([\mathrm{M}+\mathrm{H}]^{+}\right)$: 298.0986; found: 298.0992 .<smiles>CN1C(=O)C2(NC(=O)c3ccccc3N2)c2cc(Cl)ccc21</smiles>

\section{5-Chloro-1-methyl-1'H-spiro[indoline-3,2'-quinazoline]-2,4'(3'H)-dione (3p)}

Yield: $78 \%$ (245 mg). White solid, mp $276-278^{\circ} \mathrm{C} .{ }^{1} \mathrm{H}$ NMR $\left(400 \mathrm{MHz}, \mathrm{DMSO}-d_{6}\right): \delta$ $3.08\left(\mathrm{~s}, 3 \mathrm{H}, \mathrm{CH}_{3}\right), 6.61(\mathrm{~d}, 1 \mathrm{H}, \mathrm{ArH}, J=8.0 \mathrm{~Hz}), 6.73(\mathrm{t}, 1 \mathrm{H}, \mathrm{ArH}, J=6.8 \mathrm{~Hz}), 7.12(\mathrm{~d}$, $1 \mathrm{H}, \mathrm{ArH}, J=8.4 \mathrm{~Hz}), 7.26\left(\mathrm{dt}, 1 \mathrm{H}, \mathrm{ArH}, J_{1}=1.6 \mathrm{~Hz}, J_{2}=6.8 \mathrm{~Hz}\right), 7.30(\mathrm{~s}, 1 \mathrm{H}, \mathrm{NH})$, 7.51-7,53 (m, 1H, ArH), 7.56 (d, 1H, ArH, $J=2.4 \mathrm{~Hz}), 7.64$ (d, 1H, ArH, $J=7.6 \mathrm{~Hz})$, $8.34(\mathrm{~s}, 1 \mathrm{H}, \mathrm{NH}) .{ }^{13} \mathrm{C}$ NMR $\left(100 \mathrm{MHz}, \mathrm{DMSO}-d_{6}\right): \delta 26.5,71.3,111.1,114.4,114.7$, 118.0, 123.7, 125.5, 127.4, 127.5, 131.2, 133.9, 143.0, 146.9, 164.1, 174.5. HRMS (ESI) $\mathrm{m} / \mathrm{z}$ calcd for $\mathrm{C}_{16} \mathrm{H}_{13} \mathrm{ClN}_{3} \mathrm{O}_{2}\left([\mathrm{M}+\mathrm{H}]^{+}\right)$: 314.0691 ; found: 314.0695 .<smiles>O=C1NC2(Nc3ccccc31)C(=O)N(Cc1ccccc1)c1ccc(F)cc12</smiles>

1-Benzyl-5-fluoro-1'H-spiro[indoline-3,2'-quinazoline]-2,4'(3'H)-dione (3q) 
Yield: $93 \%$ (347 mg). White solid, mp 279-280 ${ }^{\circ} \mathrm{C} .{ }^{1} \mathrm{H}$ NMR (400MHz, DMSO- $\left.d_{6}\right): \delta$ 4.79-4.88 (m, 2H, $\left.\mathrm{CH}_{2}\right), 6.66(\mathrm{~d}, 1 \mathrm{H}, \mathrm{ArH}, J=8.0 \mathrm{~Hz}), 6.72(\mathrm{t}, 1 \mathrm{H}, \mathrm{ArH}, J=7.6 \mathrm{~Hz})$, $6.90\left(\mathrm{dd}, 1 \mathrm{H}, \mathrm{ArH}, J_{1}=4.0 \mathrm{~Hz}, J_{2}=8.4 \mathrm{~Hz}\right), 7.18-7.29(\mathrm{~m}, 3 \mathrm{H}, \operatorname{ArH}), 7.32-7.39(\mathrm{~m}$, 4H, ArH), 7.47 (s, 1H, NH), 7.48-7.49 (m, 1H, ArH), 7.65 (d, 1H, ArH, J=7.6Hz), $8.57(\mathrm{~s}, 1 \mathrm{H}, \mathrm{NH}) .{ }^{13} \mathrm{C} \mathrm{NMR}\left(100 \mathrm{MHz}, \mathrm{DMSO}-d_{6}\right): \delta 42.7,71.0,110.6,113.0,114.0$, $114.3,117.0,117.5,126.9,127.3,127.5,128.6,130.4,130.4,133.4,135.6,138.9$, 146.5, $158.8\left(\mathrm{~d},{ }^{l} J_{C F}=238 \mathrm{~Hz}, \mathrm{C}-\mathrm{F}\right), 163.8,174.5$. HRMS (ESI) $\mathrm{m} / \mathrm{z}$ calcd for $\mathrm{C}_{22} \mathrm{H}_{17} \mathrm{FN}_{3} \mathrm{O}_{2}\left([\mathrm{M}+\mathrm{H}]^{+}\right)$: 374.1299; found: 374.1302 .

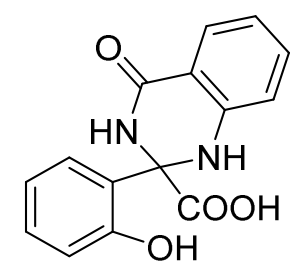

2-(2-Hydroxyphenyl)-4-oxo-1,2,3,4-tetrahydroquinazoline-2-carboxylic acid (3r')

Yield: $57 \%$ (162 mg). Yellow solid, mp $286-289^{\circ} \mathrm{C} .{ }^{1} \mathrm{H}$ NMR (400MHz, DMSO- $\left.d_{6}\right)$ : $\delta$ 6.95-7.00 (t, 2H, ArH), 7.24 (t, 1H, ArH, $J=7.6 \mathrm{~Hz}), 7.53$ (t, 1H, ArH, $J=8.4 \mathrm{~Hz})$, 7.59 (t, 1H, ArH, $J=7.6 \mathrm{~Hz}), 7.79$ (d, 1H, ArH, $J=7.6 \mathrm{~Hz}), 7.82$ (s, 1H, NH), 7.89 (d, $1 \mathrm{H}, \mathrm{ArH}, J=8.0 \mathrm{~Hz}), 8.36(\mathrm{~s}, 1 \mathrm{H}, \mathrm{NH}), 8.56$ (d, $1 \mathrm{H}, \mathrm{ArH}, J=8.4 \mathrm{~Hz}), 10.76(\mathrm{~s}, 1 \mathrm{H}$, $\mathrm{OH}), 12.63(\mathrm{~s}, 1 \mathrm{H}, \mathrm{OH}) .{ }^{13} \mathrm{C}$ NMR $\left(100 \mathrm{MHz}, \mathrm{DMSO}-d_{6}\right): \delta 117.6,119.8,120.8$, 121.2, 124.0, 129.3, 131.6, 132.9, 136.4, 139.0, 159.8, 162.6, 170.9, 191.4. Anal. Calcd for $\mathrm{C}_{15} \mathrm{H}_{12} \mathrm{~N}_{2} \mathrm{O}_{4}$ : C, 63.38; H, 4.25; N, 9.85. Found: C, 63.44; H, 4.18; N, 9.91.

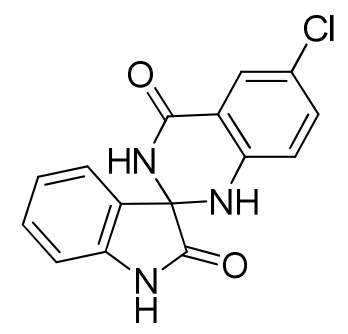

\section{6'-Chloro-1'H-spiro[indoline-3,2'-quinazoline]-2,4'(3'H)-dione (3s) ${ }^{[5]}$}

Yield: $92 \%$ (276 mg). White solid, mp 262-264 ${ }^{\circ} \mathrm{C} .{ }^{1} \mathrm{H}$ NMR (400MHz, DMSO- $\left.d_{6}\right): \delta$ $6.65(\mathrm{~d}, 1 \mathrm{H}, \operatorname{ArH}, J=8.4 \mathrm{~Hz}), 6.86(\mathrm{~d}, 1 \mathrm{H}, \operatorname{ArH}, J=7.6 \mathrm{~Hz}), 7.06$ (t, 1H, ArH, $J=$ $7.6 \mathrm{~Hz}), 7.27(\mathrm{~d}, 1 \mathrm{H}, \mathrm{ArH}, J=8.4 \mathrm{~Hz}), 7.34$ (t, 1H, ArH, $J=7.6 \mathrm{~Hz}), 7.49$ (s, 1H, NH), $7.52(\mathrm{~d}, 2 \mathrm{H}, \operatorname{ArH}, J=10.4 \mathrm{~Hz}), 8.65(\mathrm{~s}, 1 \mathrm{H}, \mathrm{NH}), 10.34(\mathrm{~s}, 1 \mathrm{H}, \mathrm{NH}) .{ }^{13} \mathrm{C} \mathrm{NMR}$ 
$\left(100 \mathrm{MHz}, \mathrm{DMSO}-d_{6}\right): \delta 71.3,110.7,116.2,116.3,121.3,122.9,126.0,126.4,129.3$, $131.5,133.5,142.7,146.1,163.4,176.3$.

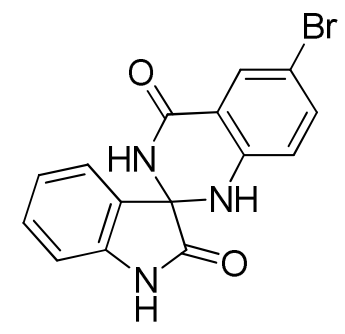

\section{6'-Bromo-1'H-spiro[indoline-3,2'-quinazoline]-2,4'(3'H)-dione (3t)}

Yield: $85 \%$ (293 mg). White solid, $\mathrm{mp}>300^{\circ} \mathrm{C} .{ }^{1} \mathrm{H}$ NMR $(400 \mathrm{MHz}, \mathrm{DMSO}-\mathrm{d} 6): \delta$ $6.60(\mathrm{~d}, 1 \mathrm{H}, \mathrm{ArH}, J=8.8 \mathrm{~Hz}), 6.86(\mathrm{~d}, 1 \mathrm{H}, \mathrm{ArH}, J=7.6 \mathrm{~Hz}), 7.06(\mathrm{t}, 1 \mathrm{H}, \mathrm{ArH}, J=$ 7.2Hz), 7.32-7.39 (m, 2H, ArH), 7.49-7.51 (d, 2H, ArH, NH), 7.85 (d, 1H, ArH, $J=$ $1.2 \mathrm{~Hz}), 8.54(\mathrm{~s}, 1 \mathrm{H}, \mathrm{NH}), 10.35(\mathrm{~s}, 1 \mathrm{H}, \mathrm{NH}) .{ }^{13} \mathrm{C} \mathrm{NMR}\left(100 \mathrm{MHz}, \mathrm{DMSO}-d_{6}\right): \delta 71.3$, $108.6,110.7,116.7,116.8,122.9,126.0,129.3,129.4,131.5,135.2,142.7,146.5$, 163.2, 176.2. HRMS (ESI) $\mathrm{m} / \mathrm{z}$ calcd for $\mathrm{C}_{15} \mathrm{H}_{11} \mathrm{BrN}_{3} \mathrm{O}_{2}\left([\mathrm{M}+\mathrm{H}]^{+}\right)$: 344.0029; found: 344.0034 .<smiles>Cc1ccc2c(c1)C(=O)NC1(C2)NC(=O)Nc2ccccc21</smiles>

6'-Methyl-1'H-spiro[indoline-3,2'-quinazoline]-2,4' (3'H)-dione (3u)

Yield: $90 \%$ (251 mg). White solid, mp $250-251^{\circ} \mathrm{C} .{ }^{1} \mathrm{H}$ NMR (400MHz, DMSO- $\left.d_{6}\right): \delta$ $2.20(\mathrm{~s}, 3 \mathrm{H}, \mathrm{CH}), 6.53$ (d, 1H, ArH, $J=8.0 \mathrm{~Hz}), 6.84$ (d, 1H, ArH, $J=7.6 \mathrm{~Hz}$ ), 7.02-7.10 (m, 3H, ArH), 7.31 (t, 1H, ArH, $J=7.6 \mathrm{~Hz}), 7.42-7.46$ (m, 3H, ArH), 8.29 (s, 1H, NH), 10.26 (s, 1H, NH). ${ }^{13} \mathrm{C}$ NMR (100MHz, DMSO-d $): \delta 20.6,71.5,110.5$, $114.5,114.9,122.7,125.8,126.3,127.3,130.0,131.2,134.5,142.6,145.0,164.6$, 176.5. HRMS (ESI) m/z calcd for $\mathrm{C}_{16} \mathrm{H}_{14} \mathrm{~N}_{3} \mathrm{O}_{2}\left([\mathrm{M}+\mathrm{H}]^{+}\right)$: 280.1081 ; found: 280.1081 . 
<smiles>COc1ccc2c(c1)C(=O)NC1(NC(=O)Nc3ccccc31)C2=O</smiles>

\section{6'-Methoxy-1'H-spiro[indoline-3,2'-quinazoline]-2,4'(3'H)-dione (3v)}

Yield: $81 \%$ (239 mg). Yellow solid, mp $276-278^{\circ} \mathrm{C} .{ }^{1} \mathrm{H}$ NMR (400MHz, DMSO- $\left.d_{6}\right)$ : $\delta 3.71\left(\mathrm{~s}, 3 \mathrm{H}, \mathrm{CH}_{3}\right), 6.58$ (d, 1H, ArH, $\left.J=8.8 \mathrm{~Hz}\right), 6.83$ (d, 1H, ArH, $\left.J=7.6 \mathrm{~Hz}\right), 6.89$ $\left(\mathrm{d}, 1 \mathrm{H}, \mathrm{ArH}, J_{1}=8.8 \mathrm{~Hz}, J_{2}=2.8 \mathrm{~Hz}\right), 6.95(\mathrm{~s}, 1 \mathrm{H}, \mathrm{NH}), 7.04(\mathrm{t}, 1 \mathrm{H}, \mathrm{ArH}, J=7.2 \mathrm{~Hz})$, $7.15(\mathrm{~d}, 1 \mathrm{H}, \operatorname{ArH}, J=2.4 \mathrm{~Hz}), 7.31(\mathrm{t}, 1 \mathrm{H}, \operatorname{ArH}, J=7.2 \mathrm{~Hz}), 7.46(\mathrm{~d}, 1 \mathrm{H}, \mathrm{ArH}, J=$ $7.2 \mathrm{~Hz}), 8.37$ (s, 1H, NH), 10.25 (s, $1 \mathrm{H}, \mathrm{NH}) .{ }^{13} \mathrm{C}$ NMR (100MHz, DMSO-d 6 ): $\delta 55.8$, $71.5,110.4,110.5,115.5,116.0,121.4,122.7,125.8,130.0,131.2,141.4,142.6$, 151.9, 164.4, 176.5. HRMS (ESI) $\mathrm{m} / \mathrm{z}$ calcd for $\mathrm{C}_{16} \mathrm{H}_{14} \mathrm{~N}_{3} \mathrm{O}_{3}\left([\mathrm{M}+\mathrm{H}]^{+}\right): 296.1030$; found: 296.1031.<smiles>O=C1Nc2ccccc2C2(N1)NC(=O)c1cc([N+](=O)[O-])ccc12</smiles>

\section{6'-Nitro-1'H-spiro[indoline-3,2'-quinazoline]-2,4'(3'H)-dione (3w)}

Yield: $38 \%(118 \mathrm{mg})$. Yellow solid, $\mathrm{mp}>300^{\circ} \mathrm{C} .{ }^{1} \mathrm{H}$ NMR $\left(400 \mathrm{MHz}, \mathrm{DMSO}-d_{6}\right): \delta$ $6.75(\mathrm{~d}, 1 \mathrm{H}, \operatorname{ArH}, J=9.2 \mathrm{~Hz}), 6.91(\mathrm{~d}, 1 \mathrm{H}, \mathrm{ArH}, J=7.6 \mathrm{~Hz}), 7.11(\mathrm{t}, 1 \mathrm{H}, \operatorname{ArH}, J=$ $7.6 \mathrm{~Hz}), 7.39$ (t, 1H, ArH, $J=7.6 \mathrm{~Hz}), 7.56$ (d, 1H, ArH, $J=7.2 \mathrm{~Hz}), 8.13$ (d, 1H, ArH, $J=7.2 \mathrm{~Hz}), 8.42(\mathrm{~s}, 1 \mathrm{H}, \mathrm{ArH}), 8.68(\mathrm{~s}, 1 \mathrm{H}, \mathrm{NH}), 8.85(\mathrm{~s}, 1 \mathrm{H}, \mathrm{NH}), 10.54$ (s, 1H, NH).

${ }^{13} \mathrm{C}$ NMR (100MHz, DMSO-d $): \delta 71.5,111.0,113.4,114.5,123.2,126.2,128.4$, 129.6, 131.9, 138.1, 142.7, 152.5, 162.4, 175.5. HRMS (ESI) $\mathrm{m} / \mathrm{z}$ calcd for $\mathrm{C}_{15} \mathrm{H}_{10} \mathrm{~N}_{4} \mathrm{O}_{4} \mathrm{Na}\left([\mathrm{M}+\mathrm{Na}]^{+}\right)$: 333.0594; found: 333.0598 .<smiles>Cc1ccc2c(c1)NC1(NC2=O)C(=O)Nc2ccccc21</smiles> 
Yield: 83\% (232 mg). Yellow solid, mp $290-291{ }^{\circ} \mathrm{C} .{ }^{1} \mathrm{H}$ NMR (400MHz, DMSO- $\left.d_{6}\right)$ : $\delta 2.19\left(\mathrm{~d}, 3 \mathrm{H}, \mathrm{CH}_{3}\right), 6.41(\mathrm{~s}, 1 \mathrm{H}, \mathrm{ArH}), 6.50(\mathrm{~d}, 1 \mathrm{H}, \mathrm{ArH}, J=6.8 \mathrm{~Hz}), 6.84(\mathrm{~d}, 1 \mathrm{H}$, $\operatorname{ArH}, J=6.8 \mathrm{~Hz}), 7.05(\mathrm{~d}, 1 \mathrm{H}, \operatorname{ArH}, J=6.4 \mathrm{~Hz}), 7.18(\mathrm{~s}, 1 \mathrm{H}, \mathrm{NH}), 7.31$ (d, 1H, ArH, $J$ $=6.4 \mathrm{~Hz}), 7.45(\mathrm{~d}, 1 \mathrm{H}, \operatorname{ArH}, J=6.4 \mathrm{~Hz}), 7.48(\mathrm{~d}, 1 \mathrm{H}, \operatorname{ArH}, J=6.4 \mathrm{~Hz}), 8.24(\mathrm{~s}, 1 \mathrm{H}$, $\mathrm{NH}), 10.27$ (s, $1 \mathrm{H}, \mathrm{NH}) .{ }^{13} \mathrm{C}$ NMR (100MHz, DMSO- $\left.d_{6}\right): \delta 21.9,71.5,110.5,112.6$, 114.5, 118.8, 122.7, 125.8, 127.4, 131.2, 142.6, 143.8, 147.3, 164.4, 176.5. HRMS (ESI) $\mathrm{m} / \mathrm{z}$ calcd for $\mathrm{C}_{16} \mathrm{H}_{14} \mathrm{~N}_{3} \mathrm{O}_{2}\left([\mathrm{M}+\mathrm{H}]^{+}\right): 280.1081$; found: 280.1083 .

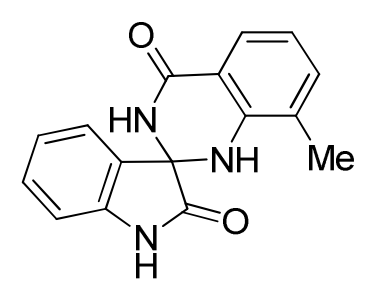

\section{8'-Methyl-1'H-spiro[indoline-3,2'-quinazoline]-2,4'(3'H)-dione (3y)}

Yield: 77\% (215 mg). Brown solid, mp 275-276 ${ }^{\circ} \mathrm{C} .{ }^{1} \mathrm{H}$ NMR (400MHz, DMSO- $\left.d_{6}\right): \delta$ 2.04 (s, 3H, $\left.\mathrm{CH}_{3}\right), 6.49$ (s, 1H, NH), 6.63 (t, 1H, ArH, J=7.2Hz), 6.82 (d, 1H, ArH, $J$ $=8.0 \mathrm{~Hz}), 7.02(\mathrm{t}, 1 \mathrm{H}, \operatorname{ArH}, J=7.2 \mathrm{~Hz}), 7.12(\mathrm{~d}, 1 \mathrm{H}, \operatorname{ArH}, J=6.8 \mathrm{~Hz}), 7.43(\mathrm{~d}, 1 \mathrm{H}$, $\operatorname{ArH}, J=7.2 \mathrm{~Hz}), 7.51$ (d, 1H, ArH, $J=7.2 \mathrm{~Hz}), 8.33$ (s, 1H, NH), 10.23 (s, 1H, NH). ${ }^{13} \mathrm{C}$ NMR $\left(100 \mathrm{MHz}, \mathrm{DMSO}-d_{6}\right): \delta 17.6,71.6,110.4,114.8,117.4,122.5,122.6$, 125.2, 125.7, 130.5, 131.0, 134.8, 142.8, 145.4, 164.5, 176.5. HRMS (ESI) m/z calcd for $\mathrm{C}_{16} \mathrm{H}_{14} \mathrm{~N}_{3} \mathrm{O}_{2}\left([\mathrm{M}+\mathrm{H}]^{+}\right)$: 280.1081; found: 280.1083 .

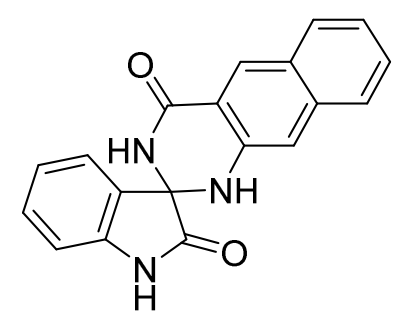

\section{H-spiro[benzo[g]quinazoline-2,3'-indoline]-2',4(3H)-dione (3z)}

Yield: 61\% (192 mg). Yellow solid, mp $>300{ }^{\circ} \mathrm{C} .{ }^{1} \mathrm{H}$ NMR (400MHz, DMSO- $\left.d_{6}\right): \delta$ $6.88(\mathrm{~d}, 1 \mathrm{H}, \mathrm{ArH}, J=8.0 \mathrm{~Hz}), 6.90(\mathrm{~s}, 1 \mathrm{H}, \mathrm{NH}), 7.08$ (t, 1H, $\operatorname{ArH}, J=7.6 \mathrm{~Hz}), 7.21(\mathrm{t}$, 1H, ArH, $J=7.6 \mathrm{~Hz}), 7.34-7.41$ (m, 3H, ArH), 5.21 (d, 1H, ArH, $J=7.2 \mathrm{~Hz}), 5.72$ (d, $1 \mathrm{H}, \operatorname{ArH}, J=8.0 \mathrm{~Hz}), 7.86$ (d, $1 \mathrm{H}, \mathrm{ArH}, J=8.4 \mathrm{~Hz}), 8.28(\mathrm{~s}, 1 \mathrm{H}, \mathrm{NH}), 8.68(\mathrm{~s}, 1 \mathrm{H}$, $\mathrm{NH}) .{ }^{13} \mathrm{C}$ NMR $\left(100 \mathrm{MHz}, \mathrm{DMSO}-d_{6}\right): \delta$ 71.2, 107.8, 110.6, 118.5, 122.8, 123.1, 
$125.8,126.0,126.1,127.3,128.5,128.6,129.3,129.7,131.4,137.1,142.8,143.9$, 164.2, 176.4. HRMS (ESI) $\mathrm{m} / \mathrm{z}$ calcd for $\mathrm{C}_{19} \mathrm{H}_{14} \mathrm{~N}_{3} \mathrm{O}_{2}\left([\mathrm{M}+\mathrm{H}]^{+}\right): 316.1081$; found: 316.1083 .<smiles>CN1C(=O)c2ccccc2NC12C(=O)Nc1ccccc12</smiles>

\section{3'-Methyl-1'H-spiro[indoline-3,2'-quinazoline]-2,4'(3'H)-dione (3aa) ${ }^{[6]}$}

Yield: $53 \%(148 \mathrm{mg})$. White solid, mp 250-252 ${ }^{\circ} \mathrm{C} .{ }^{1} \mathrm{H}$ NMR (400MHz, DMSO- $\left.d_{6}\right): \delta$ $2.54\left(\mathrm{~s}, 3 \mathrm{H}, \mathrm{CH}_{3}\right), 6.63$ (d, 1H, ArH, $\left.J=8.0 \mathrm{~Hz}\right), 6.72$ (t, 1H, ArH, $\left.J=7.6 \mathrm{~Hz}\right), 6.94$ (d, $1 \mathrm{H}, \operatorname{ArH}, J=8.0 \mathrm{~Hz}), 7.11(\mathrm{t}, 1 \mathrm{H}, \mathrm{ArH}, J=7.2 \mathrm{~Hz}), 7.25$ (t, $1 \mathrm{H}, \mathrm{ArH}, J=7.6 \mathrm{~Hz}), 7.39$ (t, 1H, ArH, $J=7.6 \mathrm{~Hz}), 7.42$ (s, 1H, NH), 7.47 (d, 1H, ArH, $J=7.2 \mathrm{~Hz}), 7.66$ (d, 1H, $\mathrm{ArH}, J=7.6 \mathrm{~Hz}), 10.57(\mathrm{~s}, 1 \mathrm{H}, \mathrm{NH}) .{ }^{13} \mathrm{C} \mathrm{NMR}\left(100 \mathrm{MHz}, \mathrm{DMSO}-d_{6}\right): \delta 28.4,75.7$, $110.7,113.7,113.8,117.4,122.7,125.2,127.1,127.5,131.2,133.2,142.0,145.7$, $163.4,174.7$.<smiles>CC(C)N1C(=O)c2ccccc2NC12C(=O)Nc1ccccc12</smiles>

\section{3'-Isopropyl-1'H-spiro[indoline-3,2'-quinazoline]-2,4'(3'H)-dione (3ab)}

Yield: 47\% (144 mg). Yellow solid, mp 91-93 ${ }^{\circ} \mathrm{C} .{ }^{1} \mathrm{H}$ NMR (400MHz, DMSO- $\left.d_{6}\right): \delta$ 1.08 (d, 6H, ArH, $J=6.8 \mathrm{~Hz}), 3.89-3.99$ (m, 1H, NH), 6.44 (d, 1H, ArH, $J=7.6 \mathrm{~Hz})$, $6.78(\mathrm{t}, 1 \mathrm{H}, \mathrm{ArH}, J=7.2 \mathrm{~Hz}), 6.94$ (d,1H, ArH, $J=7.6 \mathrm{~Hz}), 7.06$ (d, 1H, ArH, $J=$ 7.6Hz), 7.36-7.42 (m, 1H, ArH), 7.57 (t, 1H, ArH, $J=7.2 \mathrm{~Hz}), 7.78$ (d, 1H, ArH, $J=$ $7.2 \mathrm{~Hz}), 7.97(\mathrm{~d}, 1 \mathrm{H}, \mathrm{ArH}, J=7.6 \mathrm{~Hz}), 11.04(\mathrm{~s}, 1 \mathrm{H}, \mathrm{NH}) .{ }^{13} \mathrm{C} \mathrm{NMR}(100 \mathrm{MHz}$, DMSO-d $)_{6}: \delta 22.1,111.5,115.9,117.7,121.9,125.0,125.3,125.7,128.6,129.4$, 131.1, 134.7, 147.1, 147.9, 155.0, 163.2, 164.6. HRMS (ESI) m/z calcd for $\mathrm{C}_{18} \mathrm{H}_{18} \mathrm{~N}_{3} \mathrm{O}_{2}\left([\mathrm{M}+\mathrm{H}]^{+}\right): 308.1394$; found: 308.1391 . 


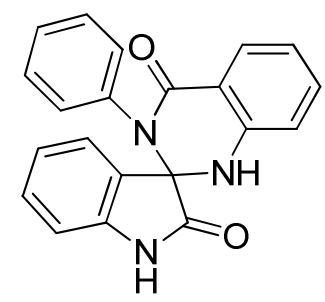

\section{3'-Phenyl-1'H-spiro[indoline-3,2'-quinazoline]-2,4'(3'H)-dione (3ac) ${ }^{[6]}$}

Yield: $78 \%$ (266 mg). White solid, mp 257-258 ${ }^{\circ} \mathrm{C} .{ }^{1} \mathrm{H}$ NMR (400MHz, DMSO- $\left.d_{6}\right): \delta$ $6.64(\mathrm{~d}, 1 \mathrm{H}, \operatorname{ArH}, J=8.0 \mathrm{~Hz}), 6.71(\mathrm{~d}, 1 \mathrm{H}, \mathrm{ArH}, J=8.0 \mathrm{~Hz}), 6.76(\mathrm{t}, 1 \mathrm{H}, \mathrm{ArH}, J=$ $8.0 \mathrm{~Hz}), 6.92$ (t, 1H, ArH, $J=7.6 \mathrm{~Hz}), 6.99$ (d, 1H, ArH, $J=7.6 \mathrm{~Hz}), 7.14$ (t, 2H, ArH, $J=7.6 \mathrm{~Hz}), 7.20(\mathrm{t}, 2 \mathrm{H}, \mathrm{ArH}, J=7.2 \mathrm{~Hz}), 7.30(\mathrm{t}, 1 \mathrm{H}, \mathrm{ArH}, J=7.6 \mathrm{~Hz}), 7.53$ (d, 1H, ArH, $J=7.6 \mathrm{~Hz}), 7.59$ (s, 1H, NH), 7.67 (d, 1H, ArH, $J=7.6 \mathrm{~Hz}), 10.37$ (s, 1H, NH). ${ }^{13} \mathrm{C}$ NMR $\left(100 \mathrm{MHz}, \mathrm{DMSO}-d_{6}\right): \delta 76.4,110.1,114.1,114.6,117.7,122.1,126.4$, $127.4,127.4,127.5,128.6,129.3,130.8,133.6,138.1,141.7,146.1,163.5,175.4$.

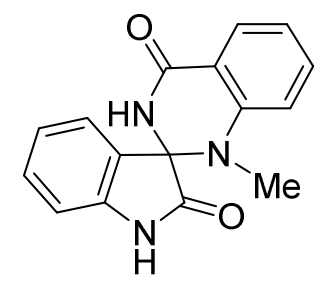

1'-Methyl-1'H-spiro[indoline-3,2'-quinazoline]-2,4'(3'H)-dione (3ad)

Yield: $67 \%(187 \mathrm{mg})$. White solid, mp $287-289^{\circ} \mathrm{C} .{ }^{1} \mathrm{H}$ NMR $\left(400 \mathrm{MHz}, \mathrm{DMSO}-d_{6}\right): \delta$ $2.42\left(\mathrm{~s}, 3 \mathrm{H}, \mathrm{CH}_{3}\right), 6.78$ (d, 1H, ArH, $\left.J=8.4 \mathrm{~Hz}\right), 6.84$ (t, $\left.1 \mathrm{H}, \mathrm{ArH}, J=7.6 \mathrm{~Hz}\right), 6.89$ (d, $1 \mathrm{H}, \mathrm{ArH}, J=8.0 \mathrm{~Hz}), 7.07$ (t, $1 \mathrm{H}, \mathrm{ArH}, J=7.2 \mathrm{~Hz}), 7.34-7.37$ (m, 2H, ArH, NH), 7.41 (t, $1 \mathrm{H}, \operatorname{ArH}, J=7.6 \mathrm{~Hz}), 7.73$ (d, 1H, ArH, $J=7.6 \mathrm{~Hz}), 8.65$ (s, 1H, NH), 10.56 (s, 1H, $\mathrm{NH}) .{ }^{13} \mathrm{C}$ NMR $\left(100 \mathrm{MHz}, \mathrm{DMSO}-d_{6}\right): \delta 31.9,76.4,111.0,112.9,116.2,118.4,123.3$, 125.2, 127.5, 128.3, 131.6, 134.4, 142.5, 148.0, 163.1, 174.6. HRMS (ESI) m/z calcd for $\mathrm{C}_{16} \mathrm{H}_{14} \mathrm{~N}_{3} \mathrm{O}_{2}\left([\mathrm{M}+\mathrm{H}]^{+}\right)$: 280.1081; found: 280.1081 .<smiles>C=CCN1c2ccccc2C(=O)NC12C(=O)Nc1ccccc12</smiles>

1'-Allyl-1'H-spiro[indoline-3,2'-quinazoline]-2,4'(3'H)-dione (3ae) 
Yield: $81 \%(247 \mathrm{mg})$. White solid, mp $277-279^{\circ} \mathrm{C} .{ }^{1} \mathrm{H}$ NMR $\left(400 \mathrm{MHz}, \mathrm{DMSO}-d_{6}\right): \delta$ 3.37-3.59 (m, 1H, $\left.\mathrm{CH}_{2}\right), 5.05$ (d, 1H, $\left.\mathrm{CH}_{2}, J=9.6 \mathrm{~Hz}\right), 5.14$ (d, 1H, $\left.\mathrm{CH}_{2}, J=16.8 \mathrm{~Hz}\right)$, 5.59-5.63 (m, 1H, CH), 6.68 (d, 1H, ArH, $J=7.6 \mathrm{~Hz}), 6.79$ (t, 1H, ArH, $J=6.4 \mathrm{~Hz})$, 6.88(d, 1H, ArH, $J=7.2 \mathrm{~Hz}), 7.06(\mathrm{t}, 1 \mathrm{H}, \mathrm{ArH}, J=6.4 \mathrm{~Hz}), 7.35(\mathrm{t}, 2 \mathrm{H}, \mathrm{ArH}, J=$ $6.8 \mathrm{~Hz}), 7.43(\mathrm{~d}, 1 \mathrm{H}, \mathrm{ArH}, J=6.8 \mathrm{~Hz}), 7.72(\mathrm{~d}, 1 \mathrm{H}, \mathrm{ArH}, J=6.8 \mathrm{~Hz}), 8.59$ (s, 1H, NH), $10.50(\mathrm{~s}, 1 \mathrm{H}, \mathrm{NH}) .{ }^{13} \mathrm{C}$ NMR $\left(100 \mathrm{MHz}, \mathrm{DMSO}-d_{6}\right): \delta 48.0,76.0,111.0,113.3,115.9$, 117.2 , 118.0, 123.0, 125.8, 127.7, 128.0, 131.7, 133.5, 134.1, 142.7, 147.1, 163.4, 175.4. HRMS (ESI) m/z calcd for $\mathrm{C}_{18} \mathrm{H}_{16} \mathrm{~N}_{3} \mathrm{O}_{2}\left([\mathrm{M}+\mathrm{H}]^{+}\right)$: 306.1237 ; found: 306.1241 .

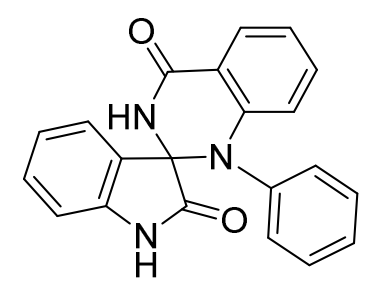

1'-Phenyl-1'H-spiro[indoline-3,2'-quinazoline]-2,4'(3'H)-dione (3af)

Yield: $85 \%$ (290 mg). White solid, mp $>300^{\circ} \mathrm{C} .{ }^{1} \mathrm{H}$ NMR $\left(400 \mathrm{MHz}, \mathrm{DMSO}-d_{6}\right): \delta$ $5.90(\mathrm{~d}, 1 \mathrm{H}, \operatorname{ArH}, J=8.0 \mathrm{~Hz}), 6.59(\mathrm{~d}, 1 \mathrm{H}, \mathrm{ArH}, J=7.6 \mathrm{~Hz}), 6.80(\mathrm{t}, 1 \mathrm{H}, \operatorname{ArH}, J=$ $7.6 \mathrm{~Hz}), 6.92$ (t, $1 \mathrm{H}, \mathrm{ArH}, J=7.6 \mathrm{~Hz}), 6.97(\mathrm{~d}, 1 \mathrm{H}, \mathrm{ArH}, J=6.8 \mathrm{~Hz}), 7.12-7.20$ (m, 3H, ArH) , 7.21-7.27 (m, 2H, ArH), 7.36 (t, 1H, ArH, 6.0Hz), 7.50 (d, 1H, ArH, 7.2Hz), $7.78(\mathrm{~d}, 1 \mathrm{H}, \operatorname{ArH}, J=6.8 \mathrm{~Hz}), 8.75(\mathrm{~s}, 1 \mathrm{H}, \mathrm{NH}), 10.40(\mathrm{~s}, 1 \mathrm{H}, \mathrm{NH}) .{ }^{13} \mathrm{C} \mathrm{NMR}$ (100MHz, DMSO- $\left.d_{6}\right): \delta 76.3,110.6,114.1,115.8,118.5,122.6,126.6,127.7,127.9$, 128.6, 129.1, 130.2, 130.4, 131.3, 132.0, 133.8, 139.9, 142.1, 148.3, 163.6, 175.8. HRMS (ESI) m/z calcd for $\mathrm{C}_{21} \mathrm{H}_{16} \mathrm{~N}_{3} \mathrm{O}_{2}\left([\mathrm{M}+\mathrm{H}]^{+}\right)$: 342.1237; found: 342.1242 .

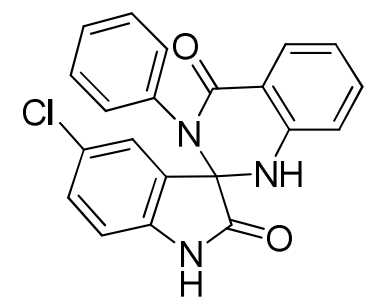

\section{5-Chloro-3'-phenyl-1'H-spiro[indoline-3,2'-quinazoline]-2,4'(3'H)-dione (3ah) ${ }^{[7]}$}

Yield: $83 \%$ (312 mg). White solid, mp $188-190^{\circ} \mathrm{C} .{ }^{1} \mathrm{H}$ NMR (400MHz, DMSO- $\left.d_{6}\right): \delta$ $6.66(\mathrm{~d}, 1 \mathrm{H}, \operatorname{ArH}, J=8.4 \mathrm{~Hz}), 6.70(\mathrm{~d}, 1 \mathrm{H}, \mathrm{ArH}, J=8.0 \mathrm{~Hz}), 6.78(\mathrm{t}, 1 \mathrm{H}, \mathrm{ArH}, J=$ 7.6Hz), 7.03 (s, 1H, NH), 7.05 (s, 1H, ArH), 7.18-7.27 (m, 4H, ArH), 7.32 (t, 1H, $\operatorname{ArH}, J=7.6 \mathrm{~Hz}), 7.65-7.68(\mathrm{~m}, 3 \mathrm{H}, \mathrm{ArH}), 10.56(\mathrm{~s}, 1 \mathrm{H}, \mathrm{NH}) .{ }^{13} \mathrm{C} \mathrm{NMR}(100 \mathrm{MHz}$, 
DMSO- $\left.d_{6}\right): \delta 76.4,111.6,114.1,114.5,117.9,126.1,126.6,127.4,127.8,128.8$, $129.1,130.7,133.8,137.9,140.6,145.8,163.3,175.3$.

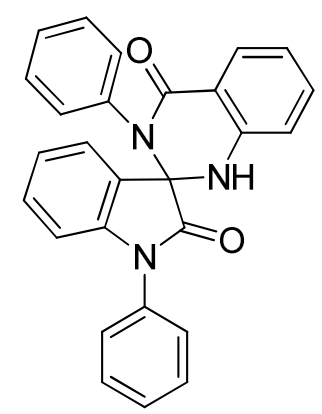

\section{1,3'-Diphenyl-1'H-spiro[indoline-3,2'-quinazoline]-2,4' (3'H)-dione (3ai)}

Yield: $91 \%$ (380 mg). White solid, mp $285-287^{\circ} \mathrm{C} .{ }^{1} \mathrm{H}$ NMR (400MHz, DMSO- $\left.d_{6}\right): \delta$ $6.52(\mathrm{~d}, 1 \mathrm{H}, \mathrm{ArH}, J=8.0 \mathrm{~Hz}), 6.77-6.83(\mathrm{~m}, 2 \mathrm{H}, \mathrm{ArH}), 7.03$ (d, 2H, ArH, $J=8.0 \mathrm{~Hz})$, 7.10 (t, 1H, ArH, $J=7.6 \mathrm{~Hz}), 7.20-7.29$ (m, 6H, ArH), 7.36 (t, 1H, ArH, $J=8.0 \mathrm{~Hz}$ ), 7.48 (t, 1H, ArH, $J=7.6 \mathrm{~Hz}), 7.58$ (t, 2H, ArH, $J=7.6 \mathrm{~Hz}), 7.73$ (t, 2H, ArH, $J=$ $8.8 \mathrm{~Hz}), 7.84(\mathrm{~s}, 1 \mathrm{H}, \mathrm{NH}) .{ }^{13} \mathrm{C} \mathrm{NMR}\left(100 \mathrm{MHz}, \mathrm{DMSO}-d_{6}\right): \delta 76.4,109.3,114.3$, $114.3,118.0,123.5,126.2,126.6,126.7,127.5,127.9,128.4,128.7,129.8,131.1$, 133.3, 133.8, 137.8, 142.8, 146.0, 163.3, 173.1. HRMS (ESI) m/z calcd for $\mathrm{C}_{27} \mathrm{H}_{20} \mathrm{~N}_{3} \mathrm{O}_{2}\left([\mathrm{M}+\mathrm{H}]^{+}\right)$: 418.1550; found: 418.1554 .<smiles>O=C(c1ccccc1)N1Cc2ccccc2NC12C(=O)Nc1ccccc12</smiles>

\section{3'-Benzoyl-3',4'-dihydro-1'H-spiro[indoline-3,2'-quinazolin]-2-one (3aj)}

Yield: 48\% (171 mg). Orange solid, mp 180-182 ${ }^{\circ} \mathrm{C} .{ }^{1} \mathrm{H}$ NMR (400MHz, DMSO- $\left.d_{6}\right)$ : $\delta 4.29\left(\mathrm{~s}, 2 \mathrm{H}, \mathrm{CH}_{2}\right), 6.25$ (d, 1H, ArH, $\left.J=6.4 \mathrm{~Hz}\right), 6.64$ (s, 1H, ArH), 6.87 (s, 2H, ArH), 7.24-7.46 (m, 7H, ArH), 7.66 (d, 2H, ArH, $J=6.4 \mathrm{~Hz}), 8.76$ (s, 1H, NH), 10.94 $(\mathrm{s}, 1 \mathrm{H}, \mathrm{NH}) .{ }^{13} \mathrm{C} \mathrm{NMR}\left(100 \mathrm{MHz}, \mathrm{DMSO}-d_{6}\right): \delta 111.8,116.7,116.9,122.2,125.2$, 125.7, 127.6, 128.5, 129.4, 131.4, 134.7, 134.8, 147.4, 149.2, 156.2, 163.9, 166.6. HRMS (ESI) m/z calcd for $\mathrm{C}_{22} \mathrm{H}_{18} \mathrm{~N}_{3} \mathrm{O}_{2}\left([\mathrm{M}+\mathrm{H}]^{+}\right)$: 356.1394; found: 356.1399 . 
<smiles>O=C1Nc2ccccc2C12Nc1ccccc1S(=O)(=O)N2</smiles>

2H,4H-spiro[benzo[e] $[1,2,4]$ thiadiazine-3,3'-indolin]-2'-one 1,1-dioxide (3ak) ${ }^{[8]}$

Yield: 68\% (205 mg). Yellow solid, mp $264-267^{\circ} \mathrm{C} .{ }^{1} \mathrm{H}$ NMR (400MHz, DMSO- $\left.d_{6}\right)$ : $\delta 6.82(\mathrm{~s}, 1 \mathrm{H}, \mathrm{ArH}), 6.84(\mathrm{~s}, 1 \mathrm{H}, \mathrm{ArH}), 6.88(\mathrm{~d}, 1 \mathrm{H}, \mathrm{ArH}, J=7.6 \mathrm{~Hz}), 7.02(\mathrm{t}, 1 \mathrm{H}, \mathrm{ArH}$, $J=7.6 \mathrm{~Hz}), 7.32$ (t, 1H, ArH, $J=7.6 \mathrm{~Hz}), 7.35$ (t, 1H, ArH, $J=8.4 \mathrm{~Hz}), 7.53$ (d, 1H, $\operatorname{ArH}, J=7.2 \mathrm{~Hz}), 7.57$ (d, 1H, ArH, $J=7.2 \mathrm{~Hz}), 7.62$ (s, 1H, NH), 8.30 (s, 1H, NH), $10.63(\mathrm{~s}, 1 \mathrm{H}, \mathrm{NH}) .{ }^{13} \mathrm{C}$ NMR $\left(100 \mathrm{MHz}, \mathrm{DMSO}-d_{6}\right): \delta 72.5,110.5,116.8,117.9$, $122.4,123.3,123.7,126.8,129.2,131.0,133.5,141.7,143.8,173.8$.<smiles>Cc1ccc(N2C(=O)c3ccccc3NC23C(=O)N(C)c2ccc(Br)cc23)cc1</smiles>

5-Bromo-1-methyl-3'-(p-tolyl)-1'H-spiro[indoline-3,2'-quinazoline]-2,4'(3'H)-dio ne (3al) ${ }^{[9]}$

Yield: 81\% (363 mg). Yellow solid, mp 286-288 ${ }^{\circ} \mathrm{C} .{ }^{1} \mathrm{H}$ NMR (400MHz, DMSO- $\left.d_{6}\right)$ : $\delta 2.18\left(\mathrm{~s}, 3 \mathrm{H}, \mathrm{CH}_{3}\right), 3.01\left(\mathrm{~s}, 3 \mathrm{H}, \mathrm{CH}_{3}\right), 6.68(\mathrm{~d}, 1 \mathrm{H}, \mathrm{ArH}, J=8.0 \mathrm{~Hz}), 6.68(\mathrm{t}, 1 \mathrm{H}, \mathrm{ArH}$, $J=7.6 \mathrm{~Hz}), 6.85(\mathrm{~d}, 1 \mathrm{H}, \operatorname{ArH}, J=8.4 \mathrm{~Hz}), 7.02(\mathrm{~d}, 1 \mathrm{H}, \mathrm{ArH}, J=8.0 \mathrm{~Hz}), 7.32(\mathrm{t}, 1 \mathrm{H}$, $\operatorname{ArH}, J=7.6 \mathrm{~Hz}), 7.45$ (d, 1H, ArH, $J=8.4 \mathrm{~Hz}), 7.62$ (s, 1H, ArH), 7.68 (d, 1H, ArH, $J$ $=7.6 \mathrm{~Hz}), 7.82(\mathrm{~s}, 1 \mathrm{H}, \mathrm{NH}) .{ }^{13} \mathrm{C} \mathrm{NMR}\left(100 \mathrm{MHz}, \mathrm{DMSO}-d_{6}\right): \delta 21.0,26.6,76.5,111.6$, $114.6,115.0,118.5,128.0,129.4,129.5,129.8,134.1,134.2,135.6,137.6,143.0$, $146.2,163.9,174.0$.

5. Characterization data of compounds 5, 7, 9, 11, 13, 16 and 18<smiles>O=C1Nc2ccccc2C12N=c1ccccc1=N2</smiles> 


\section{Spiro[benzo[d] imidazole-2,3'-indolin]-2'-one (5) ${ }^{[10]}$}

Yield: 85\% (200 mg). White solid, mp 290-292 ${ }^{\circ} \mathrm{C} .{ }^{1} \mathrm{H}$ NMR (400MHz, DMSO- $\left.d_{6}\right): \delta$ 7.36-7.52 (m, 4H, ArH), 7.66 (t, 1H, ArH, $J=7.6 \mathrm{~Hz}), 7.87$ (d, 1H, $\mathrm{ArH}, J=8.0 \mathrm{~Hz}$ ), $8.32(\mathrm{~d}, 1 \mathrm{H}, \operatorname{ArH}, J=8.0 \mathrm{~Hz}), 8.37$ (d, $1 \mathrm{H}, \mathrm{ArH}, J=7.6 \mathrm{~Hz}), 11.96(\mathrm{~s}, 1 \mathrm{H}, \mathrm{NH}) .{ }^{13} \mathrm{C}$ NMR (100MHz, DMSO-d $\left.d_{6}\right): \delta 111.8,114.7,115.9,119.1,123.3,123.6,124.4,125.0$, $130.6,132.2,137.1,143.5,146.4,147.7$.<smiles>O=C1NC2(N=C3CCCCC3=N2)c2ccccc21</smiles>

\section{4,5,6,7-Tetrahydrospiro[benzo[d]imidazole-2,3'-indolin]-2'-one (7)}

Yield: 53\% (127 mg). White solid, mp 275-276 ${ }^{\circ} \mathrm{C} .{ }^{1} \mathrm{H}$ NMR (400MHz, DMSO- $\left.d_{6}\right): \delta$ 1.91-1.92 (m, 4H, $\left.\mathrm{CH}_{2}\right), 3.00-3.05$ (m, 4H, $\left.\mathrm{CH}_{2}\right), 7.24-7.28$ (m, 1H, ArH), 7.53 (d, 1H, $\operatorname{ArH}, J=4.0 \mathrm{~Hz}), 8.13(\mathrm{~d}, 1 \mathrm{H}, \mathrm{ArH}, J=7.6 \mathrm{~Hz}), 11.76(\mathrm{~s}, 1 \mathrm{H}, \mathrm{NH}) .{ }^{13} \mathrm{C} \mathrm{NMR}$ (100MHz, DMSO-d $): \delta$ 23.0, 23.2, 32.2, 32.7, 112.4, 119.8, 120.5, 121.0, 133.0, 140.6, 144.3, 144.5, 149.3. HRMS (ESI) $\mathrm{m} / \mathrm{z}$ calcd for $\mathrm{C}_{14} \mathrm{H}_{14} \mathrm{~N}_{3} \mathrm{O}\left([\mathrm{M}+\mathrm{H}]^{+}\right)$: 240.1131; found: 240.1133 .<smiles>O=C1Nc2ccc(Cl)cc2C12Nc1cccc3cccc(c13)N2</smiles>

\section{5-Chloro-1'H,3'H-spiro[indoline-3,2'-perimidin]-2-one (9) ${ }^{[11]}$}

Yield: $37 \%$ (59.5 mg). Yellow brown, $\mathrm{mp}>300^{\circ} \mathrm{C} .{ }^{1} \mathrm{H}$ NMR $\left(400 \mathrm{MHz}, \mathrm{DMSO}-d_{6}\right): \delta$ $6.46(\mathrm{~d}, 2 \mathrm{H}, \operatorname{ArH}, J=7.2 \mathrm{~Hz}), 6.88$ (d, 1H, ArH, $J=8.4 \mathrm{~Hz}), 7.02$ (d, 2H, ArH, $J=$ 8.4Hz), 7.14-7.18 (t, 4H, ArH, NH), 7.29 (s, 1H, ArH), 7.38 (d, 1H, ArH, J=8.0Hz), $10.40(\mathrm{~s}, 1 \mathrm{H}, \mathrm{NH}) .{ }^{13} \mathrm{C}$ NMR $\left(100 \mathrm{MHz}, \mathrm{DMSO}-d_{6}\right): \delta 77.5,141.9,140.6,134.0$, $132.2,130.6,127.3,126.2,125.6,116.3,111.8,111.7,105.7,68.1$. 


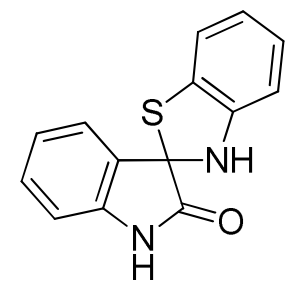

\section{H-Spiro[benzo[d]thiazole-2,3'-indolin]-2'-one (11) ${ }^{[12]}$}

Yield: $16 \%$ (40.7 mg). Yellow solid, mp 205-207 ${ }^{\circ} \mathrm{C} .{ }^{1} \mathrm{H}$ NMR (400MHz, DMSO- $\left.d_{6}\right)$ : $\delta 6.55(\mathrm{~d}, 1 \mathrm{H}, \mathrm{ArH}, J=7.6 \mathrm{~Hz}), 6.64(\mathrm{t}, 1 \mathrm{H}, \mathrm{ArH}, J=7.2 \mathrm{~Hz}), 6.86(\mathrm{~d}, 1 \mathrm{H}, \mathrm{ArH}, J=$ 7.6Hz), 6.92 (t, 1H, ArH, $J=7.6 \mathrm{~Hz}), 7.05$ (d, 1H, ArH, $J=8.0 \mathrm{~Hz}), 7.06$ (t, 1H, ArH, $J=8.0 \mathrm{~Hz}), 7.28(\mathrm{~s}, 1 \mathrm{H}, \mathrm{NH}), 7.30(\mathrm{t}, 1 \mathrm{H}, \mathrm{ArH}, J=7.6 \mathrm{~Hz}), 7.57$ (d, $1 \mathrm{H}, \operatorname{ArH}, J=$ 7.2Hz), $10.37(\mathrm{~s}, 1 \mathrm{H}, \mathrm{NH}) .{ }^{13} \mathrm{C} \mathrm{NMR}\left(100 \mathrm{MHz}, \mathrm{CDCl}_{3}+\mathrm{DMSO}_{-} d_{6}\right): \delta 74.3,108.4$, $110.0,118.6,121.1,122.4,124.1,125.6,125.7,129.8,130.5,141.3,147.2,176.1$.<smiles>O=C1Nc2ccc(Cl)cc2C12NCCc1c2[nH]c2ccccc12</smiles>

\section{5-Chloro-2',3',4',9'-tetrahydrospiro[indoline-3,1'-pyrido[3,4-b]indol]-2-one} (13) $)^{[13]}$

Yield: 55\% (178 mg). White solid, mp 280-282 ${ }^{\circ} \mathrm{C} .{ }^{1} \mathrm{H}$ NMR (400MHz, DMSO- $\left.d_{6}\right): \delta$ 2.70-2.85 (m, 2H, $\left.\mathrm{CH}_{2}\right), 3.09-3.17\left(\mathrm{~m}, 2 \mathrm{H}, \mathrm{CH}_{2}\right), 3.58(\mathrm{~s}, 1 \mathrm{H}, \mathrm{NH}), 6.95(\mathrm{~d}, 1 \mathrm{H}, J=$ $8.4 \mathrm{~Hz}, \mathrm{ArH}), 6.98$ (t, 1H, J=7.2Hz, ArH), 7.04 (t, 1H, J=7.2Hz, ArH), 7.09 (s, 1H, $\operatorname{ArH}), 7.18(\mathrm{~d}, 1 \mathrm{H}, J=8.0 \mathrm{~Hz}, \mathrm{ArH}), 7.32\left(\mathrm{dd}, 1 \mathrm{H}, \mathrm{ArH}, \quad J_{1}=8.4 \mathrm{~Hz}, J_{2}=2.0 \mathrm{~Hz}\right)$, $7.47(\mathrm{~d}, 1 \mathrm{H}, J=8.0 \mathrm{~Hz}, \operatorname{ArH}), 10.48$ (s, 1H, NH), 10.58 (s, 1H, NH). ${ }^{13} \mathrm{C} \mathrm{NMR}$ (100MHz, DMSO- $\left.d_{6}\right): \delta 21.4,61.3,110.6,111.1,111.4,117.9,118.5,121.3,124.6$, $125.7,126.5,128.9,130.8,134.6,136.1,141.2,178.1$.<smiles>O=C1Nc2ccccc2C1=O</smiles>

Indoline-2,3-dione (16) ${ }^{[14]}$

Yield: 37\% (54.4 mg). Red solid, mp 200-201 ${ }^{\circ} \mathrm{C} .{ }^{1} \mathrm{H}$ NMR (400MHz, DMSO- $\left.d_{6}\right): \delta$ $6.91(\mathrm{~d}, 1 \mathrm{H}, \mathrm{J}=8.0 \mathrm{~Hz}, \operatorname{ArH}), 7.07$ (t, 1H, J = 7.6Hz, ArH), 7.50 (d, 1H, J = 7.2Hz, 
ArH), $11.05(\mathrm{~s}, 1 \mathrm{H}, \mathrm{NH}) ;{ }^{13} \mathrm{C}$ NMR $\left(100 \mathrm{MHz}, \mathrm{DMSO}-d_{6}\right): \delta$ 112.2, 117.8, 122.7, 124.6, 138.3, 150.7, 159.3, 184.4 .<smiles>O=C1Nc2ccccc2/C1=N/c1ccc(Br)cc1</smiles>

(E)-3-((4-Bromophenyl)imino)indolin-2-one (18) ${ }^{[15]}$

Total yield: $41 \%(E: Z=78: 22,123 \mathrm{mg})$. Yellow solid, mp $239-241{ }^{\circ} \mathrm{C} ;{ }^{1} \mathrm{H}$ NMR (400MHz, DMSO-d6): $\delta 6.44$ (d, $1 \mathrm{H}, \mathrm{ArH}, J=7.6 \mathrm{~Hz}), 6.78$ (t, $1 \mathrm{H}, \mathrm{ArH}, J=7.6 \mathrm{~Hz}$ ), $6.90(\mathrm{~d}, 1 \mathrm{H}, \mathrm{ArH}, J=8.0 \mathrm{~Hz}), 6.99$ (d, 2H, ArH, $J=8.0 \mathrm{~Hz}), 7.37$ (t, 1H, ArH, $J=7.6$ $\mathrm{Hz}), 7.66(\mathrm{~d}, 2 \mathrm{H}, \mathrm{ArH}, J=8.4 \mathrm{~Hz}), 11.03(\mathrm{~s}, 1 \mathrm{H}, \mathrm{NH}),{ }^{13} \mathrm{C}$ NMR $(100 \mathrm{MHz}$, DMSO-d $)$ : $\delta 112.1,116.1,117.6,120.2,121.7,122.4,125.9,131.6,133.0,135.2$, 147.6, 150.1, 155.8, 163.8 .

\section{References}

[1] M. M. Mane, D. M. Pore, J. Chem. Sci. 2016, 128, 657-662.

[2] K. Revathy, A. Lalitha, J. Iranian Chem. Soc. 2015, 12, 2045-2049.

[3] Y. Jiang, Y. Liu, S.-J. Tu, F. Shi, Tetrahedron: Asymmetry 2013, 24, 1286-1296.

[4] D. Rambabu, S. K. Kumar, B. Y. Sreenivas, S. Sandra, A. Kandale, P. Misra, M. V. B. Rao, M. Pal, Tetrahedron Lett. 2013, 54, 495-501.

[5] Y. Hu, M.-M. Wang, H. Chen, D.-Q. Shi, Tetrahedron 2011, 67, 9342-9346.

[6] L.-L. Wang, T. Jiang, P.-H. Li, R.-J. Sun, Z. Zuo, Adv. Syn. Catal. 2018, 360, $4832-4836$.

[7] X. Yang, X. Wang, T. Wang, W. Wang, J. Zhang, Y. Ma, Chem. Res. Chinese U. 2019, 35, 33-40.

[8] S. K. Kumar, D. Rambabu, C. H. V. Kumar, B. Y. Sreenivas, K. R. S. Prasad, M. V. B. Rao, M. Pal, RSC Adv. 2013, 3, 24863-24867.

[9] K. Engen, J. Sävmarker, U. Rosenström, J. Wannberg, T. Lundbäck, A. Jenmalm-Jensen, M. Larhed. Org. Process Res. Dev. 2014, 18, 1582-1588.

[10]A. V. Ivashchenko, A. G. Drushlyak, V. V. Titov, Khimiya Geterotsiklicheskikh Soedinenii, 1984, 5, 667-672. 
[11]S. S. Panda, R. A. Jones, P. Bachawala, P. P. Mohapatra, Mini-Rev. Med. Chem. 2017, 17, 1515-1536.

[12]R. Jain, K. Sharma, D. Kumar, Tetrahedron Lett. 2012, 53, 6236-6240.

[13]T. D. Ashton, S. M. Devine, J. J. Möhrle, B. Laleu, J. N. Burrows, S. A. Charman, D. J. Creek, B. E. Sleebs, J. Med. Chem. 2019, 62, 10526-10562.

[14]H. Zhang, Y. Wang, L. Huang, L. Zhu, Y. Feng, Y. Lu, Q. Zhao, X. Wang, Z. Wang, Chem. Commun. 2018, 54, 8265-8268.

[15]A. A. Akaev, S. I. Bezzubov, V. G. Desyatkin, N. S. Vorobyeva, A. G. Majouga, M. Ya. Melnikov, E. M. Budynina, J. Org. Chem. 2019, 84, 3340-3356. 


\section{Spectroscopic data for compounds 3a-3al}

${ }^{1} \mathrm{H}$ NMR spectrum of Compound 3a (DMSO- $\left.d_{6}, 400 \mathrm{MHz}\right)$

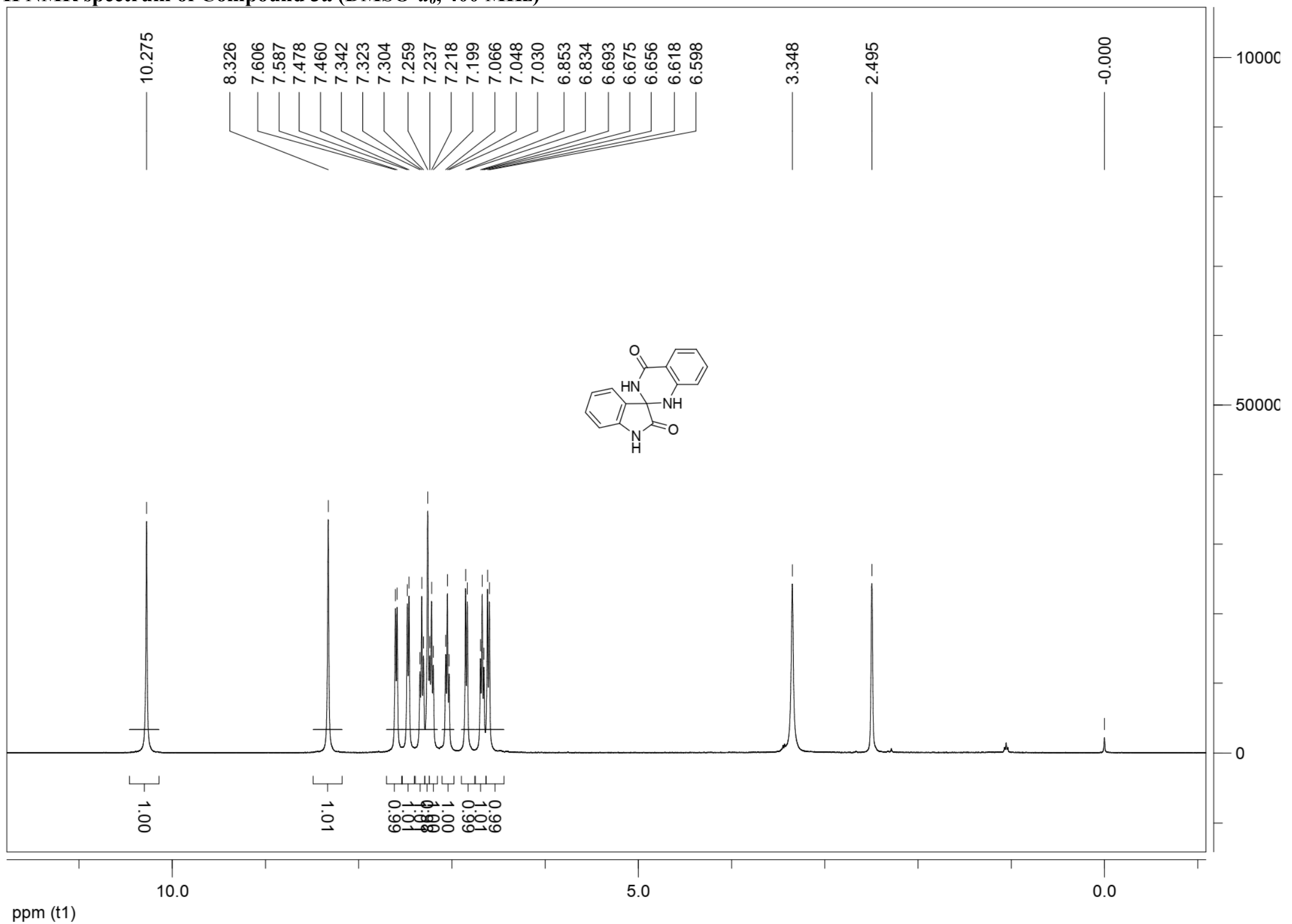


${ }^{13} \mathrm{C}$ NMR spectrum of Compound 3a (DMSO- $\left.d_{6}, 100 \mathrm{MHz}\right)$

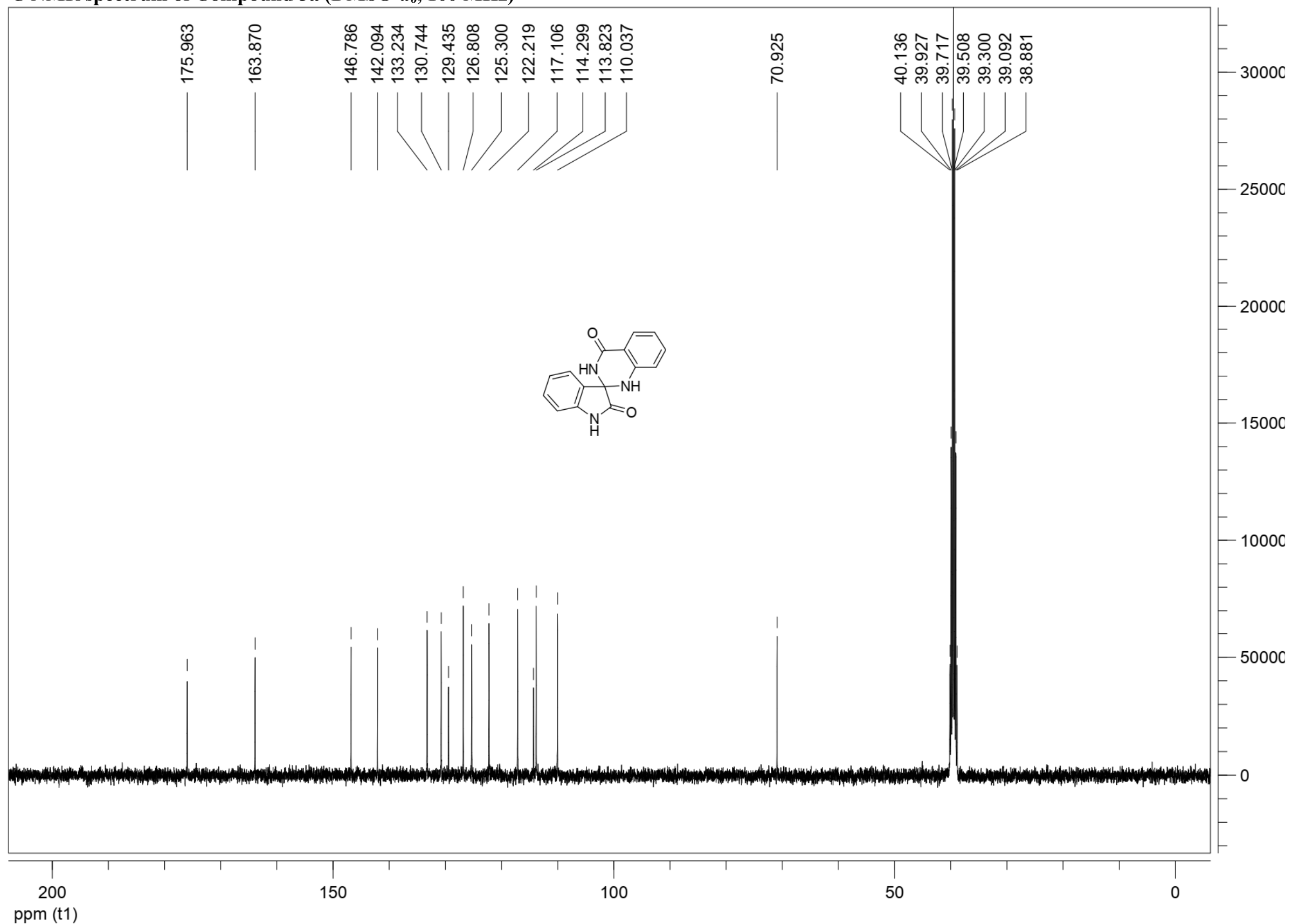




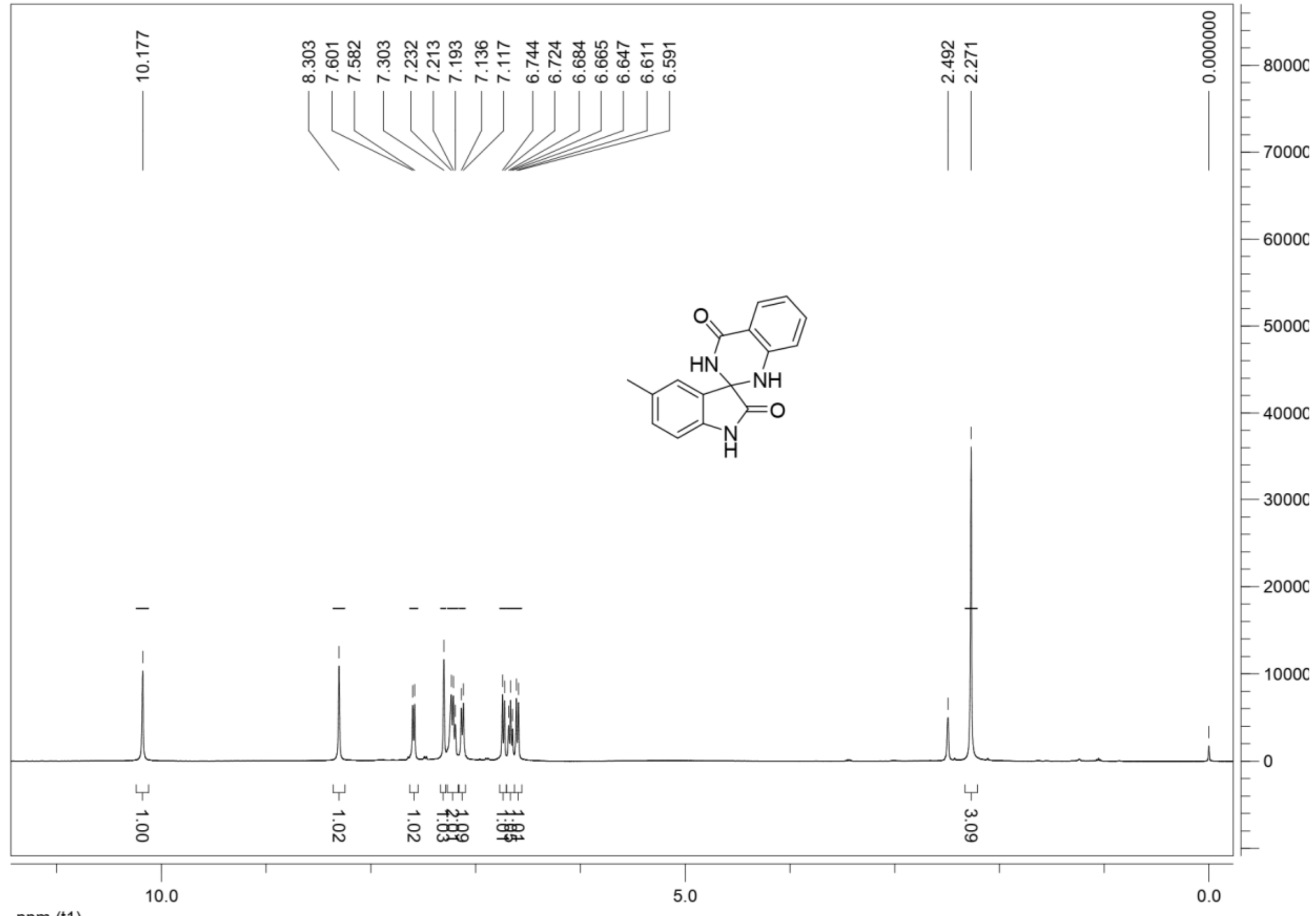


${ }^{13} \mathrm{C}$ NMR spectrum of Compound $3 \mathrm{~b}$ (DMSO- $\left.d_{6}, 100 \mathrm{MHz}\right)$

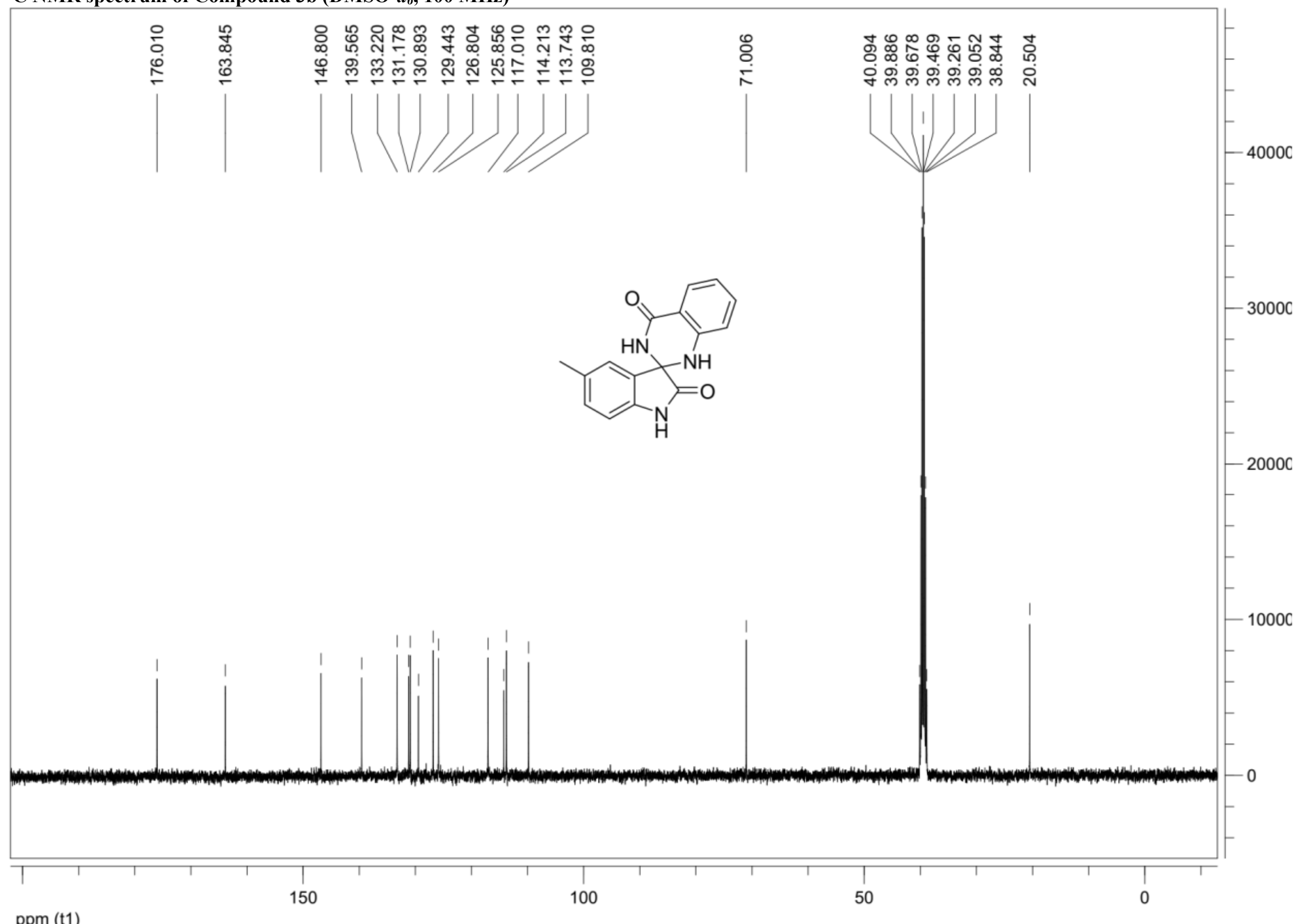


${ }^{1} \mathrm{H}$ NMR spectrum of Compound 3c (DMSO- $\left.d_{6}, 400 \mathrm{MHz}\right)$

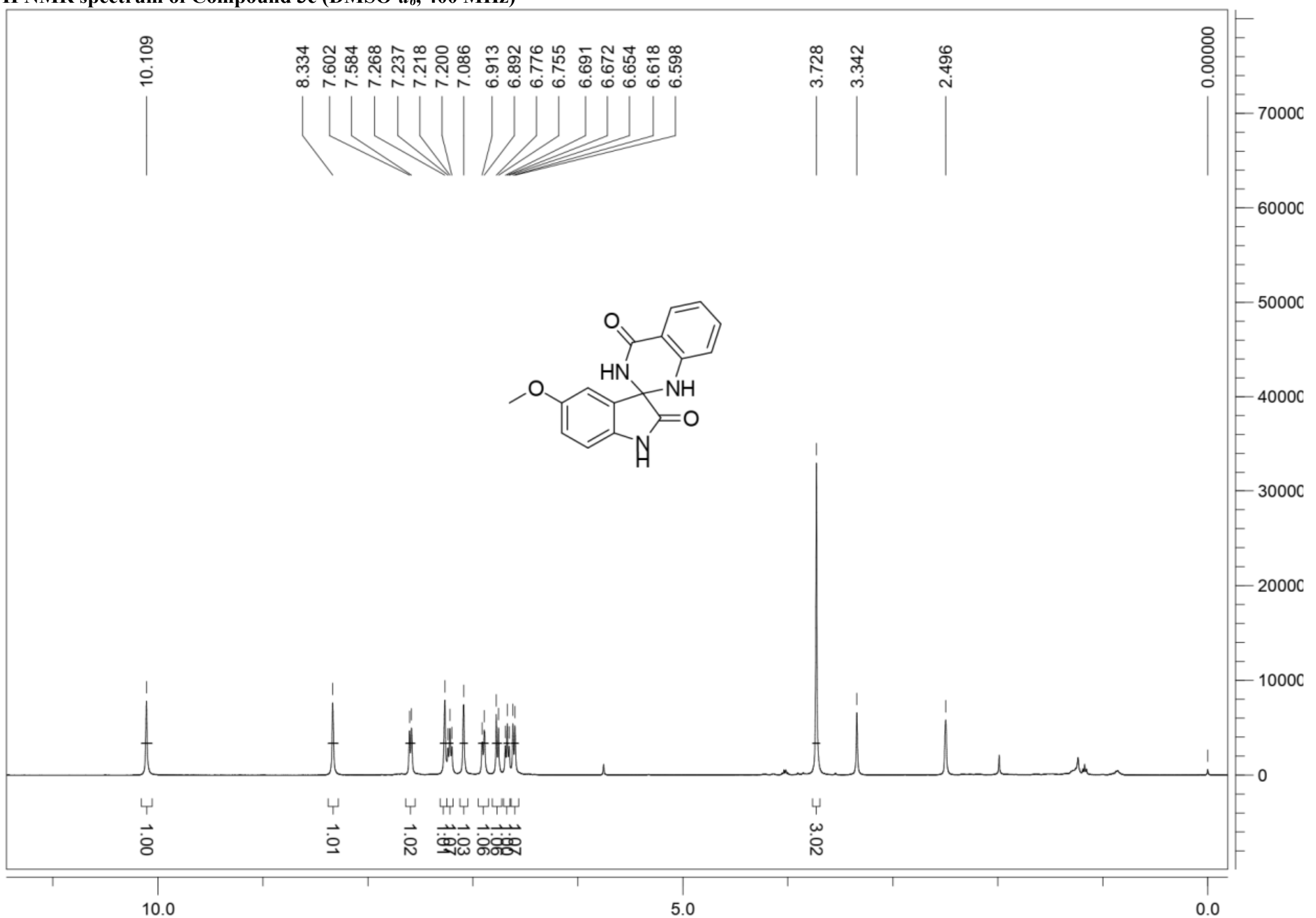

ppm (t1) 


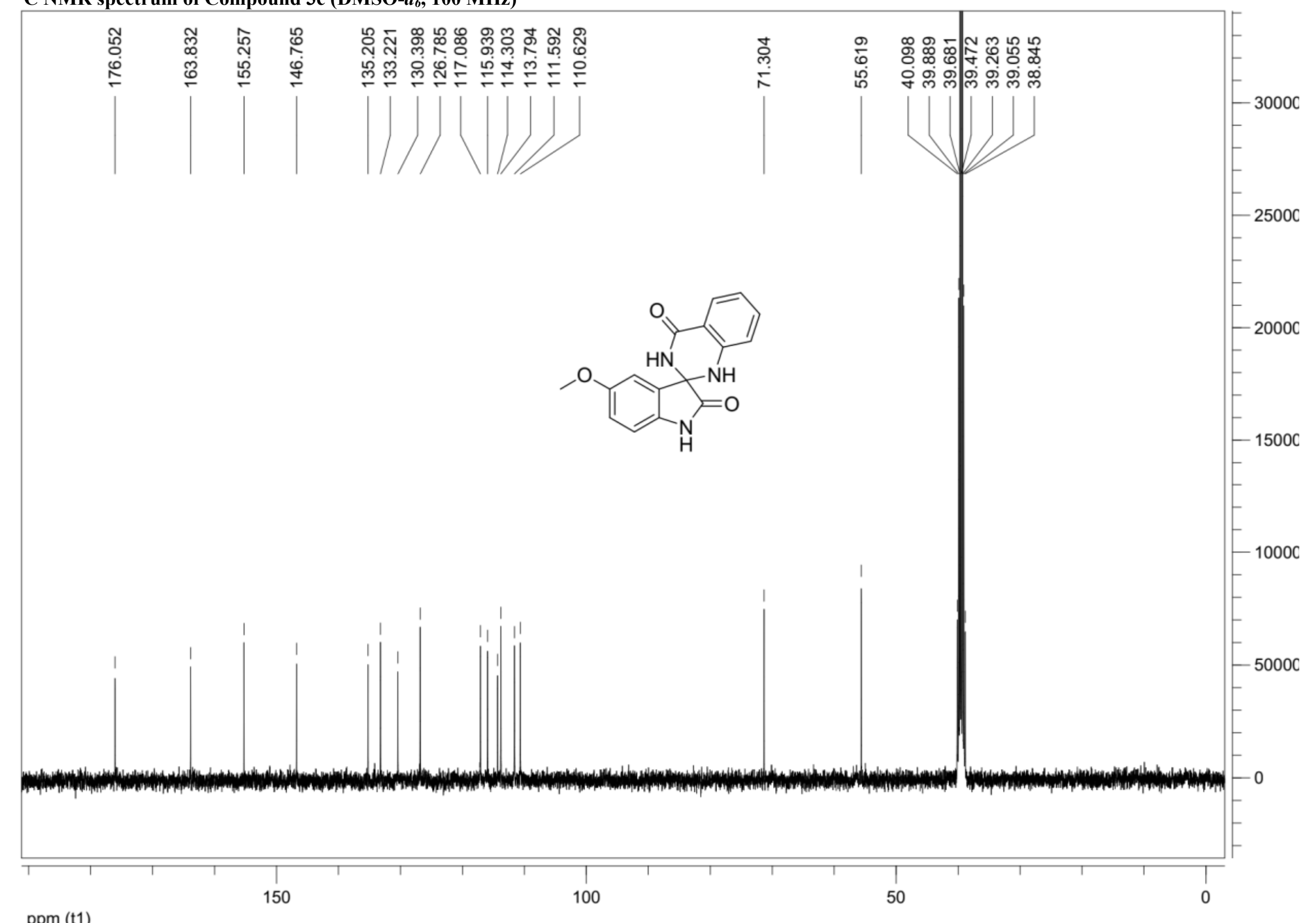

ppm (t1) 
${ }^{1} \mathrm{H}$ NMR spectrum of Compound 3d (DMSO-d $6,400 \mathrm{MHz}$ )

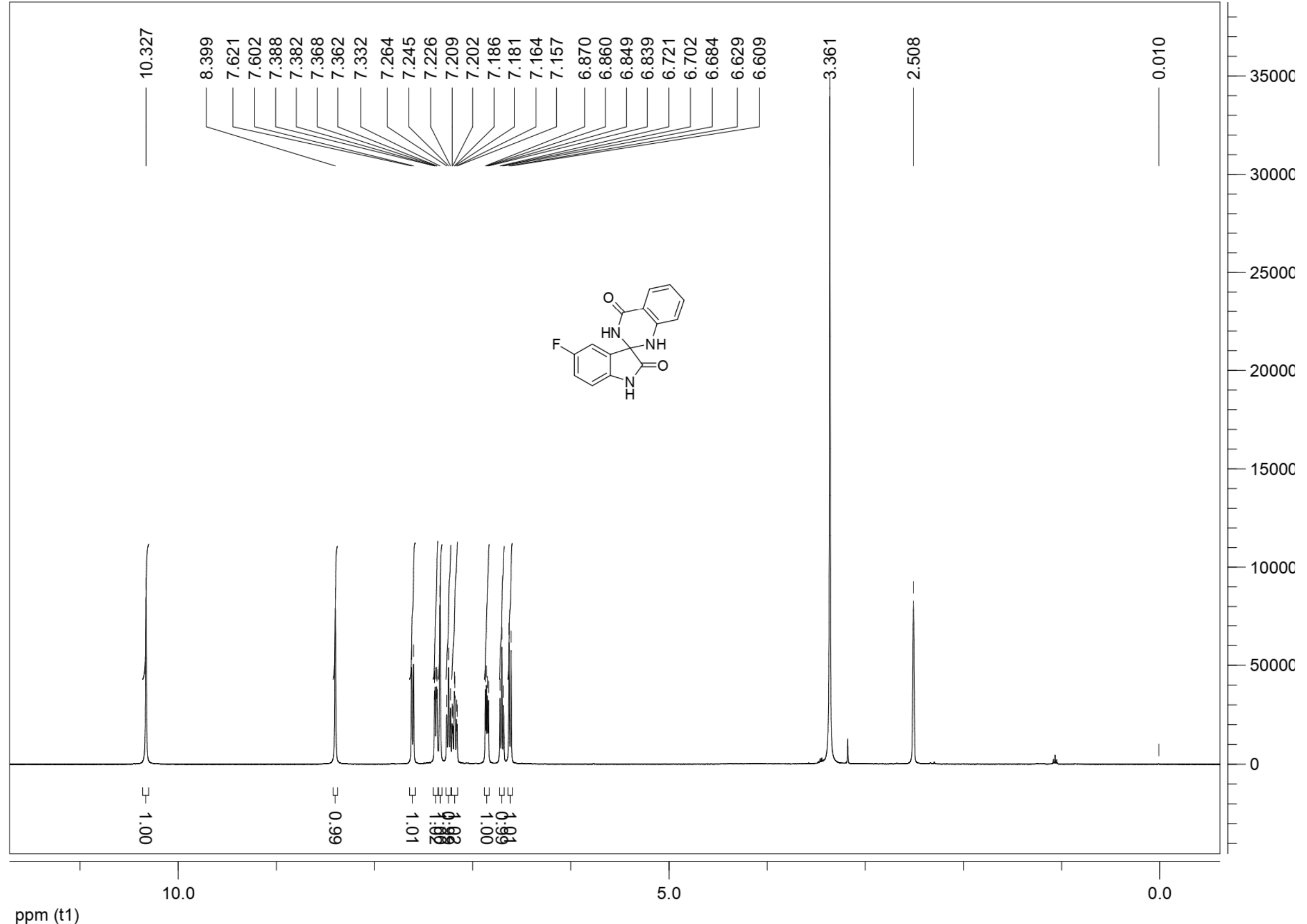




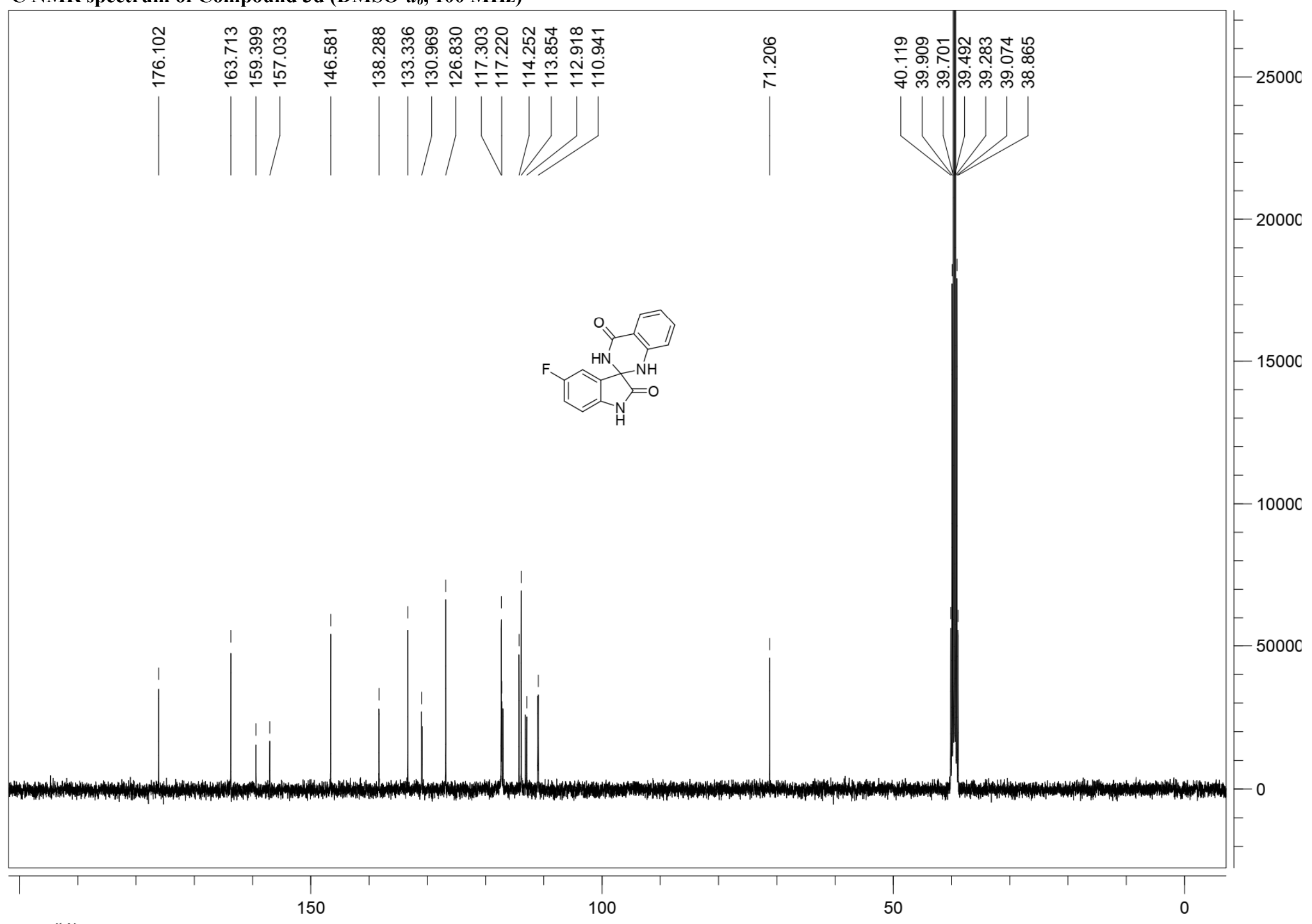

ppm (t1) 
${ }^{1} \mathrm{H}$ NMR spectrum of Compound 3e (DMSO- $d_{6}, 400 \mathrm{MHz}$ )

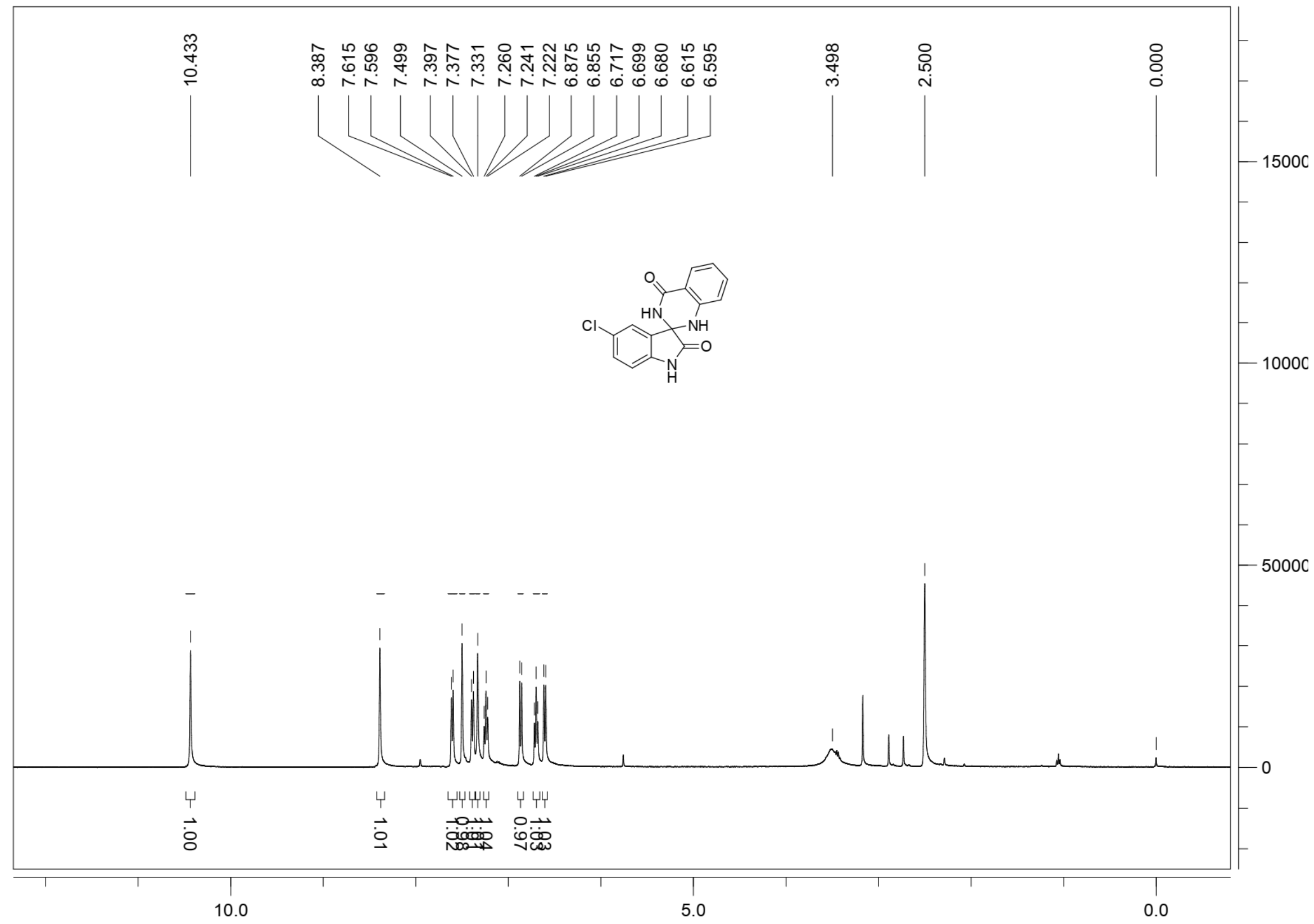

ppm (t1) 
${ }^{13} \mathrm{C}$ NMR spectrum of Compound $3 \mathrm{e}$ (DMSO- $\left.d_{6}, 100 \mathrm{MHz}\right)$

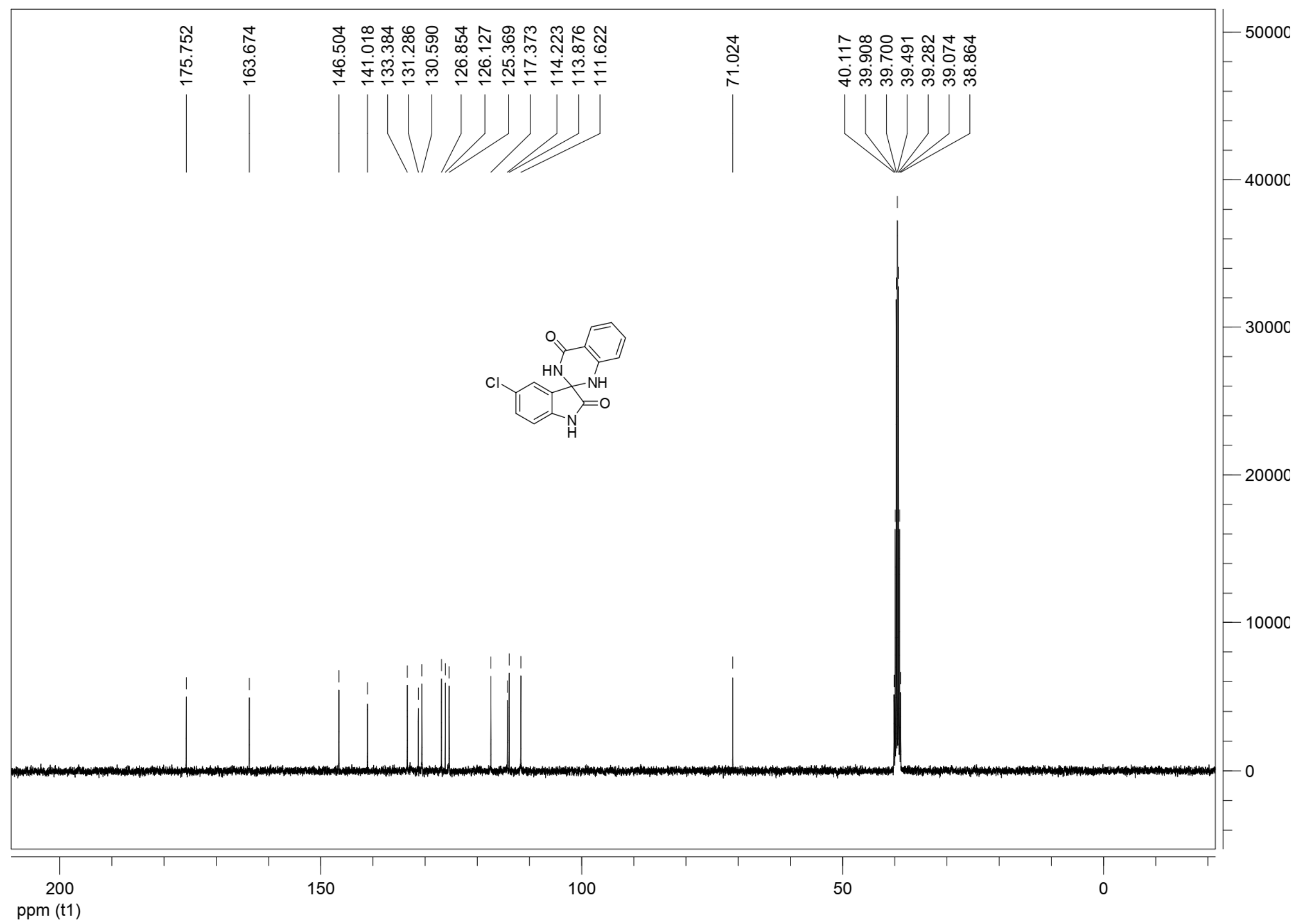


${ }^{1} \mathrm{H}$ NMR spectrum of Compound 3f (DMSO- $\left.d_{6}, 400 \mathrm{MHz}\right)$

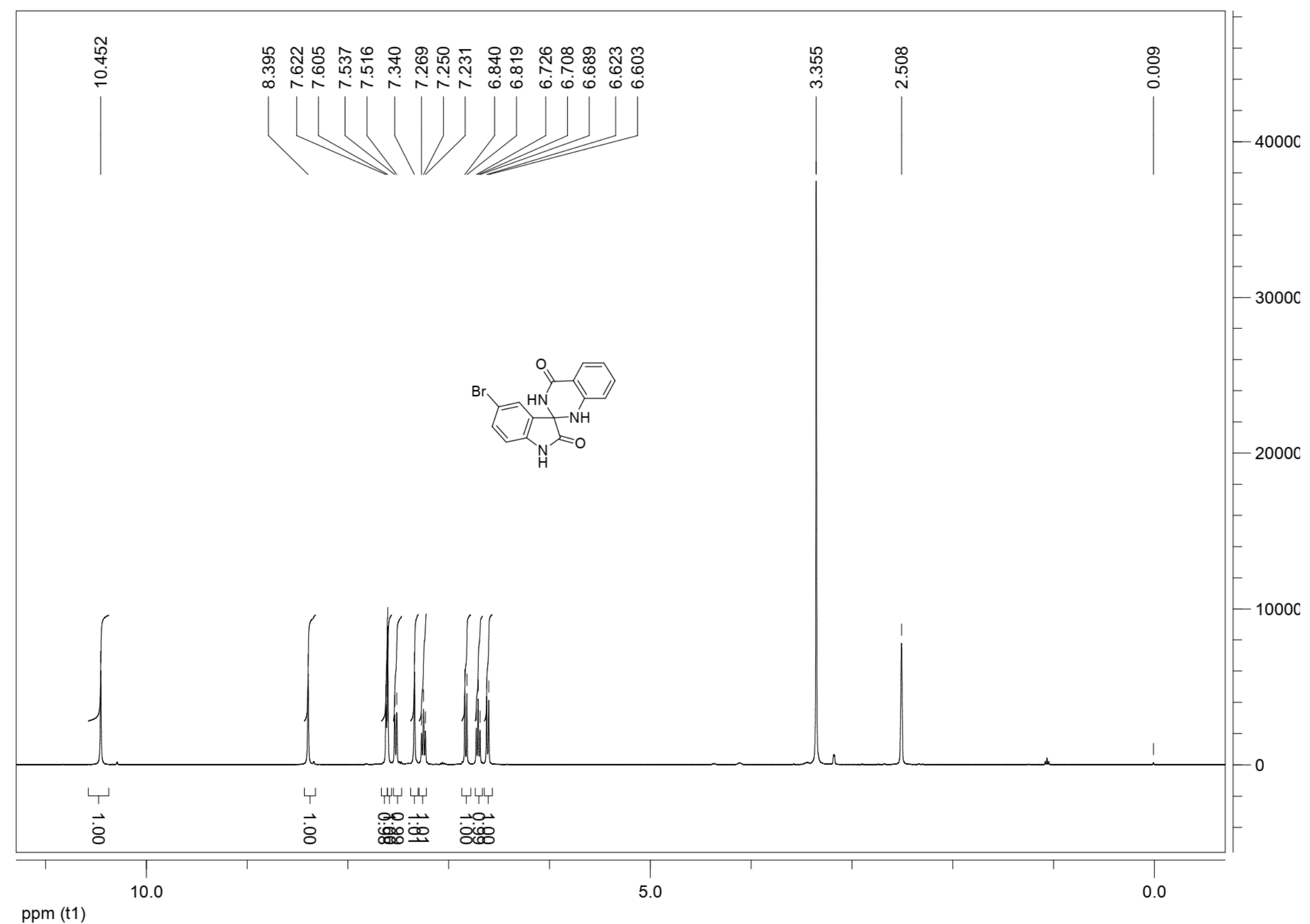


${ }^{13} \mathrm{C}$ NMR spectrum of Compound $3 \mathrm{f}$ (DMSO- $d_{6}, 100 \mathrm{MHz}$ )

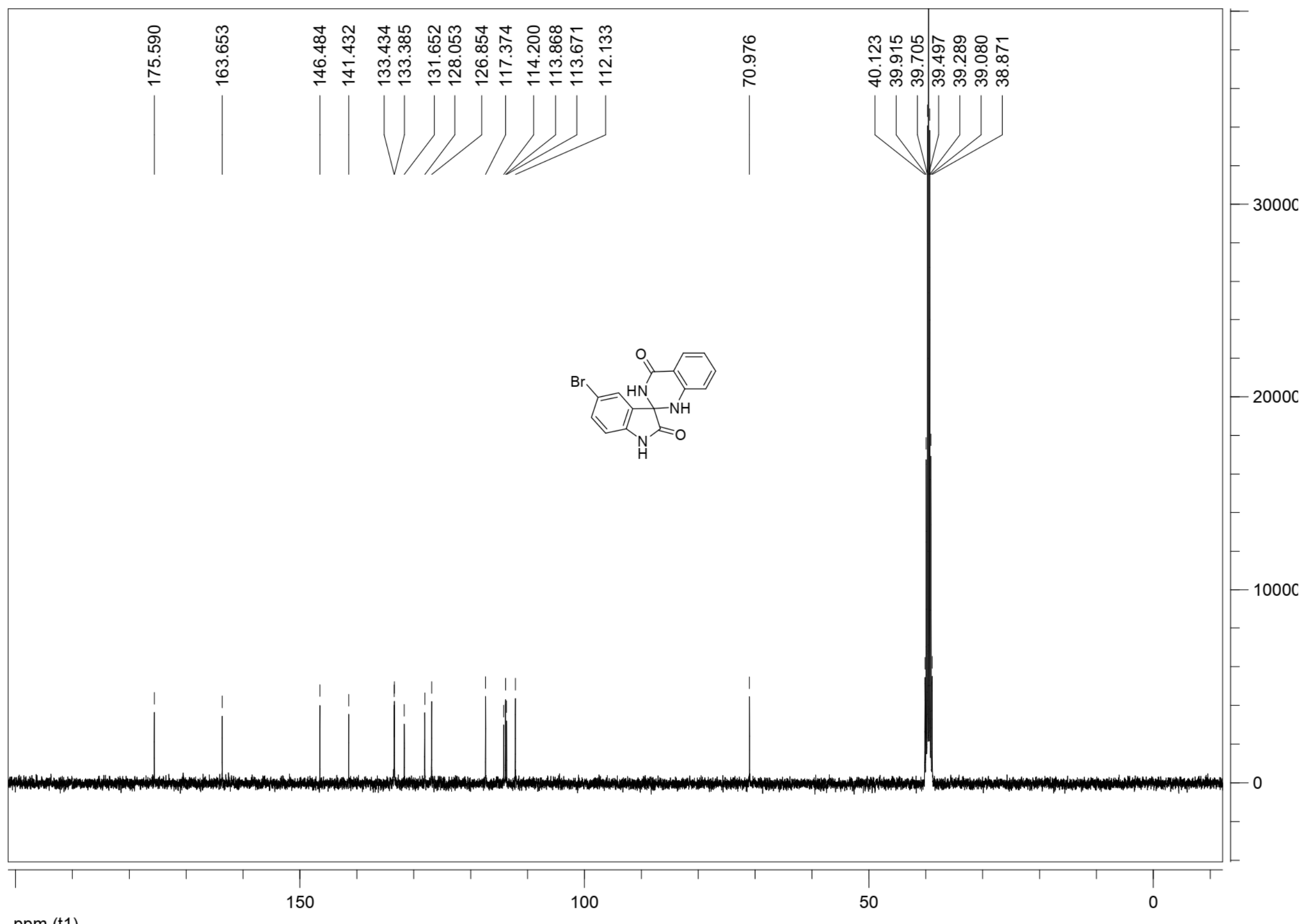

ppm (t1) 
${ }^{1} \mathrm{H}$ NMR spectrum of Compound 3g (DMSO- $\left.d_{6}, 400 \mathrm{MHz}\right)$

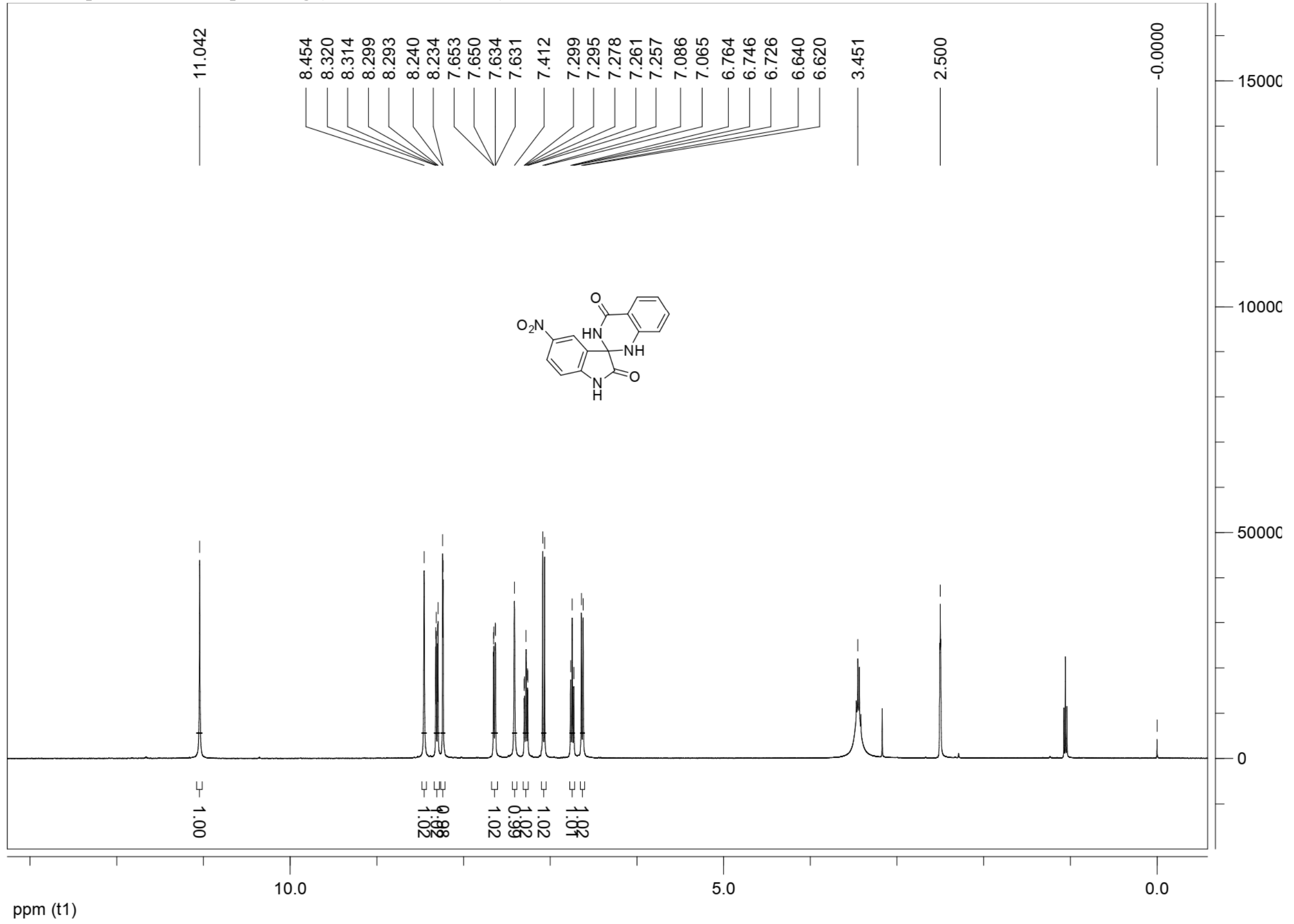


${ }^{13} \mathrm{C}$ NMR spectrum of Compound 3g (DMSO- $d_{6}, 100 \mathrm{MHz}$ )

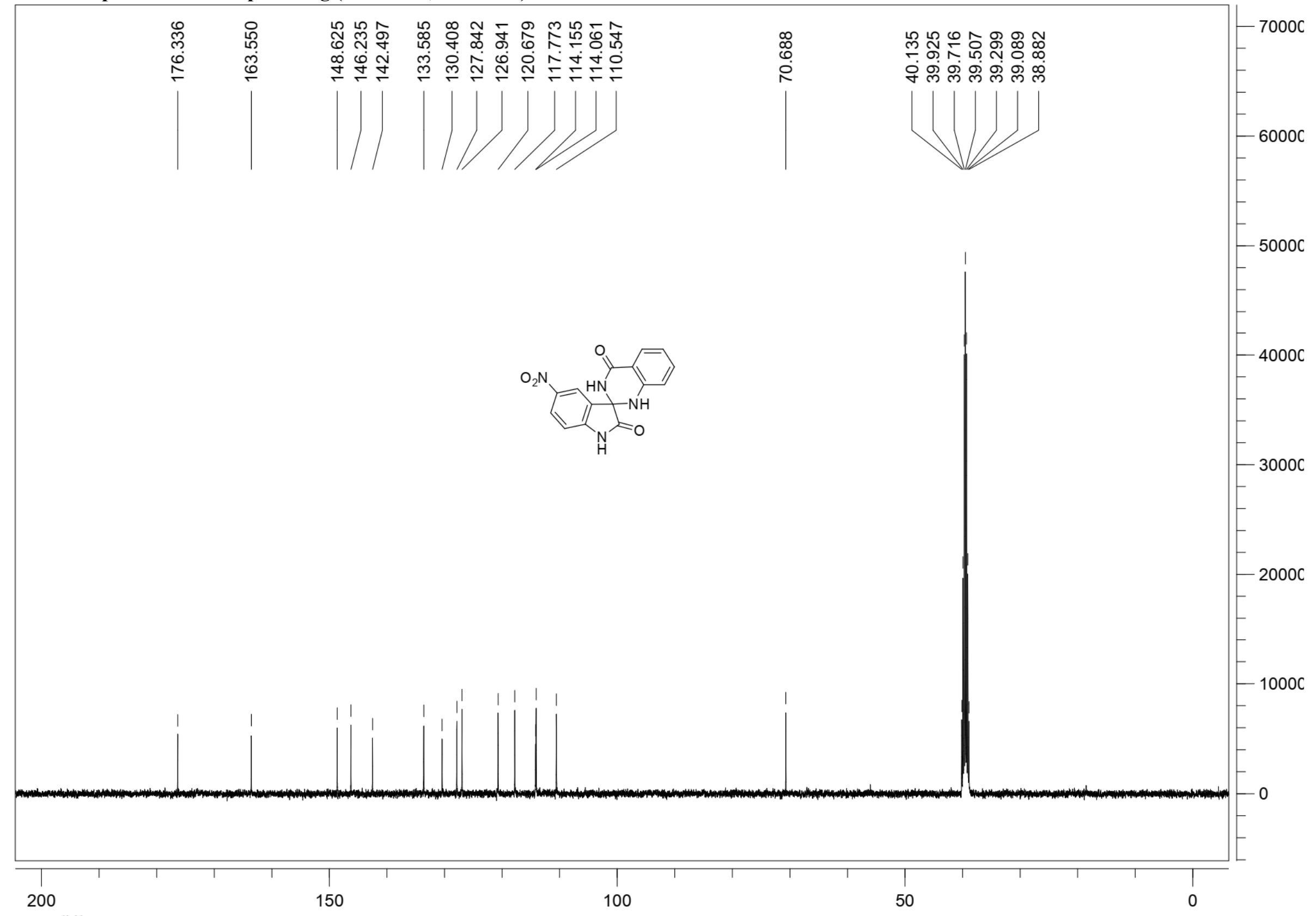

ppm (t1) 


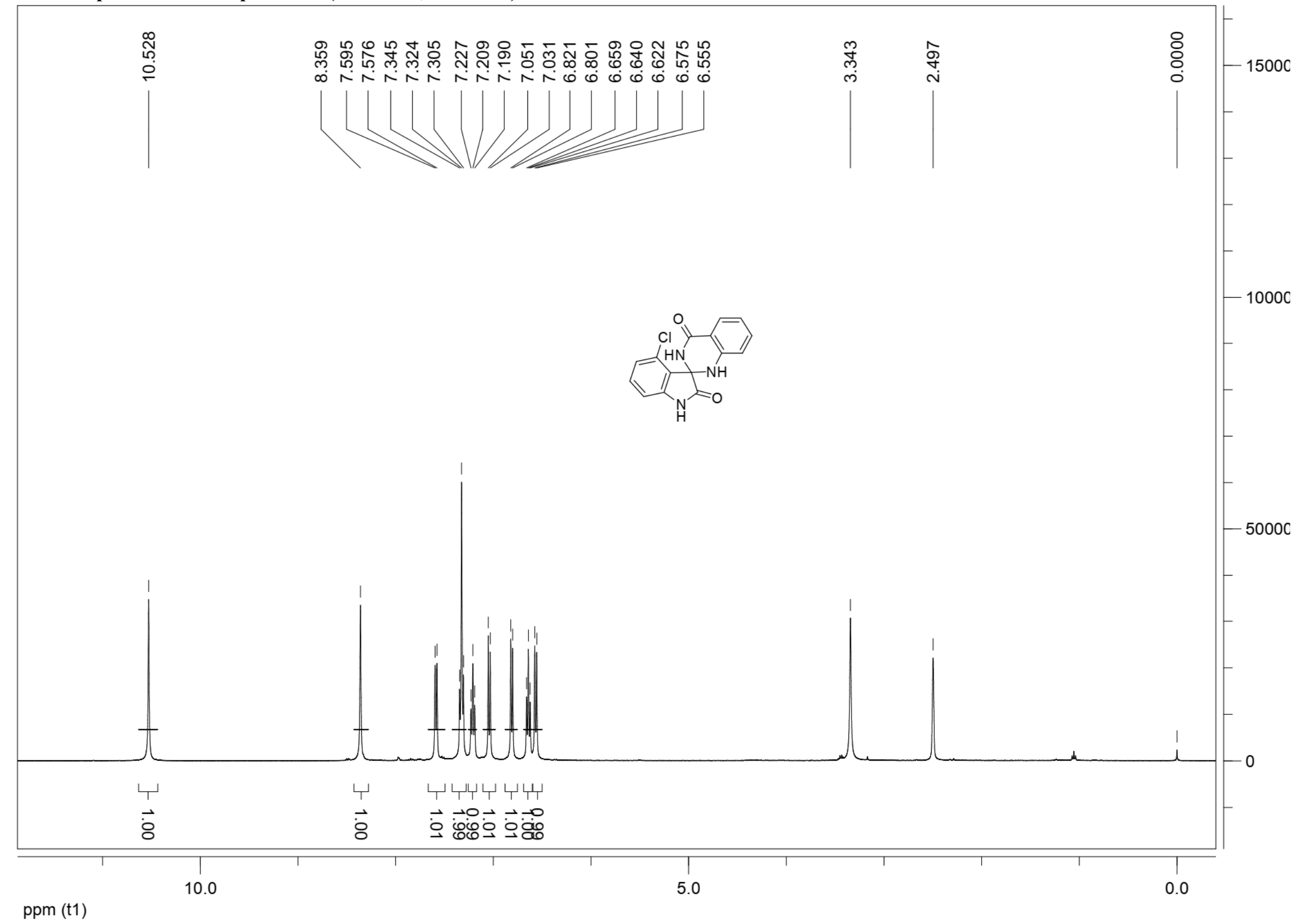




\section{${ }^{13} \mathrm{C}$ NMR spectrum of Compound $3 \mathrm{~h}$ (DMSO- $d_{6}, 100 \mathrm{MHz}$ )}

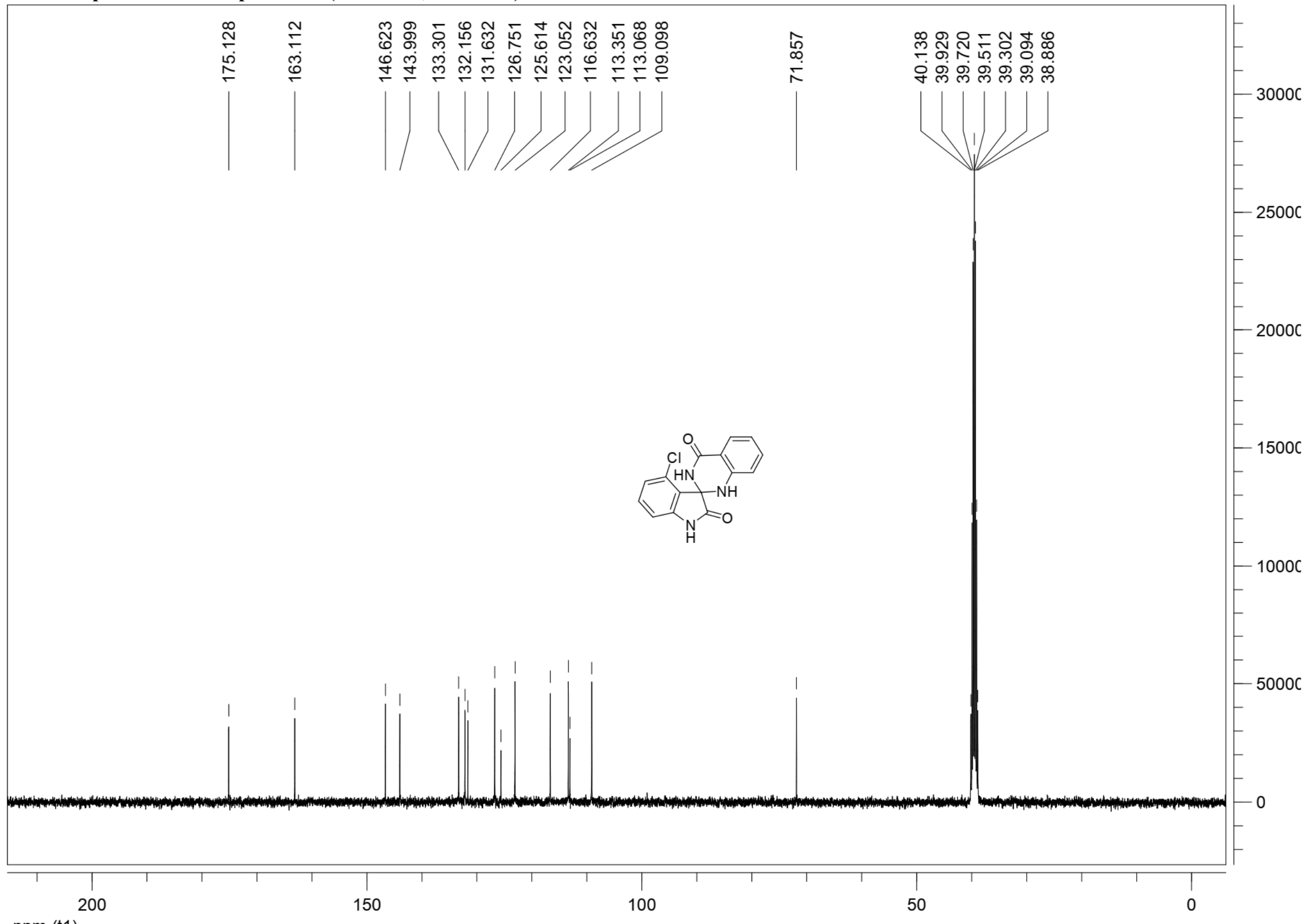

ppm (t1) 


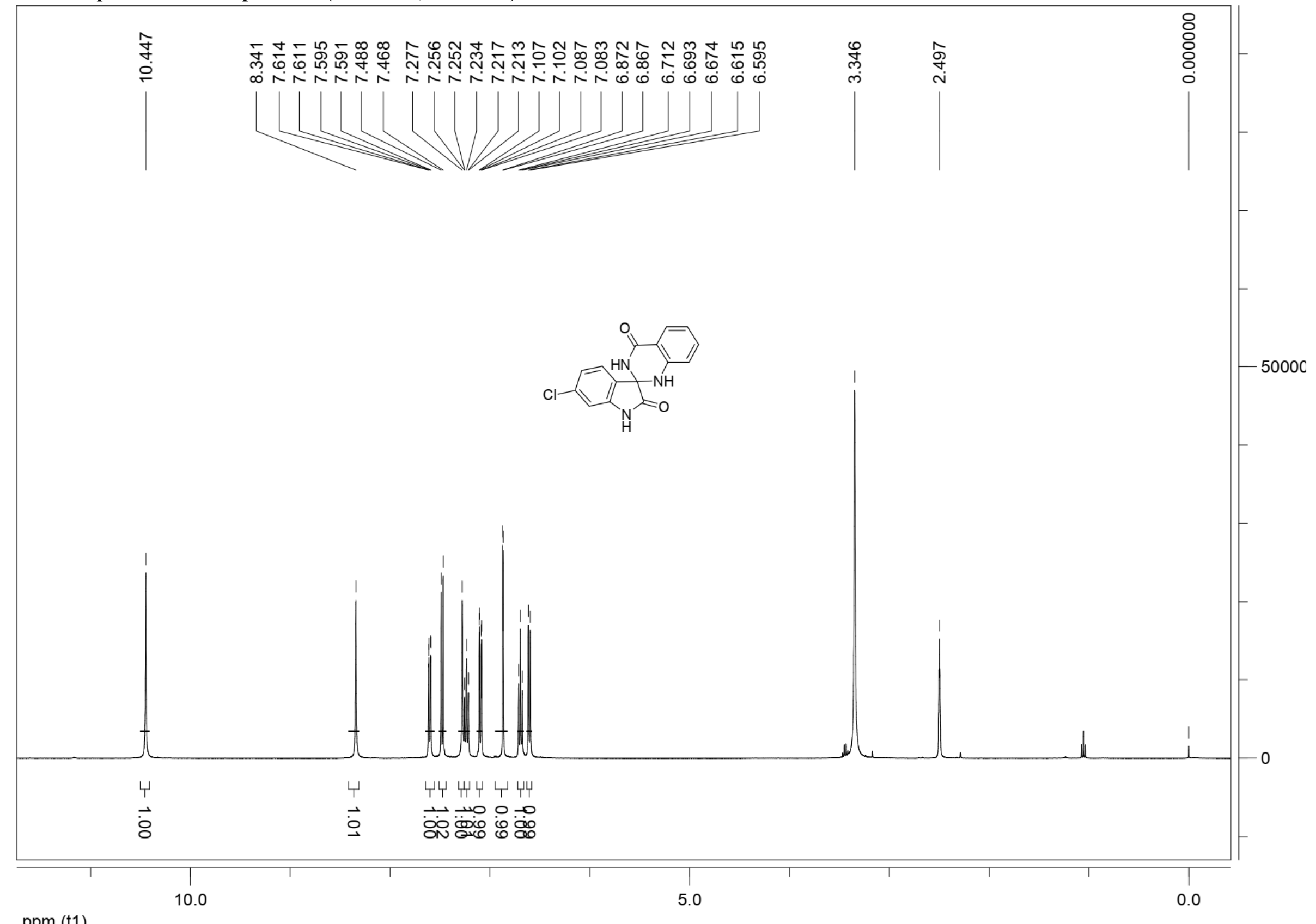




\section{${ }^{13} \mathrm{C}$ NMR spectrum of Compound 3i (DMSO- $d_{6}, 100 \mathrm{MHz}$ )}

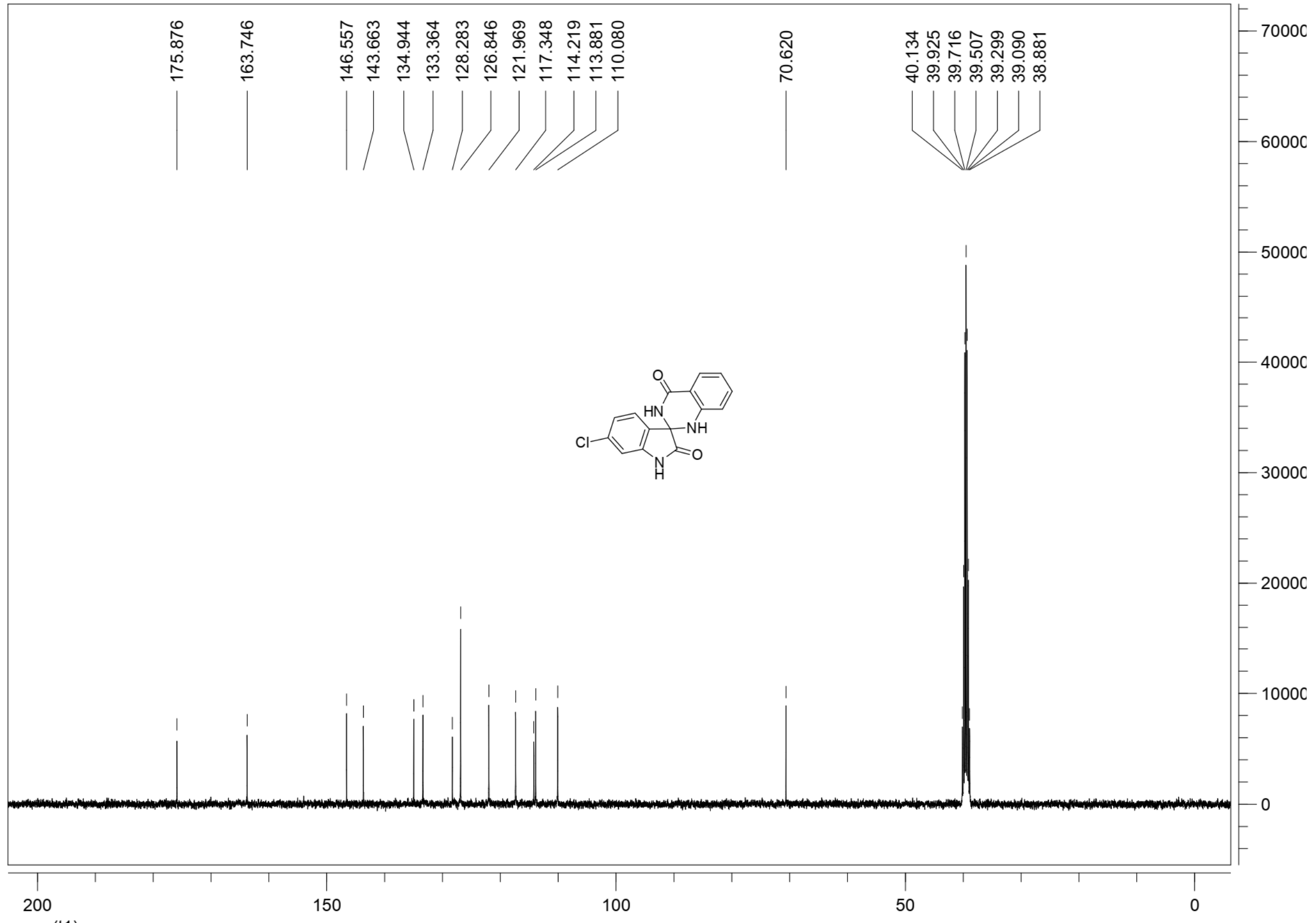

ppm (t1) 
${ }^{1} \mathrm{H}$ NMR spectrum of Compound 3j (DMSO- $d_{6}, 400 \mathrm{MHz}$ )

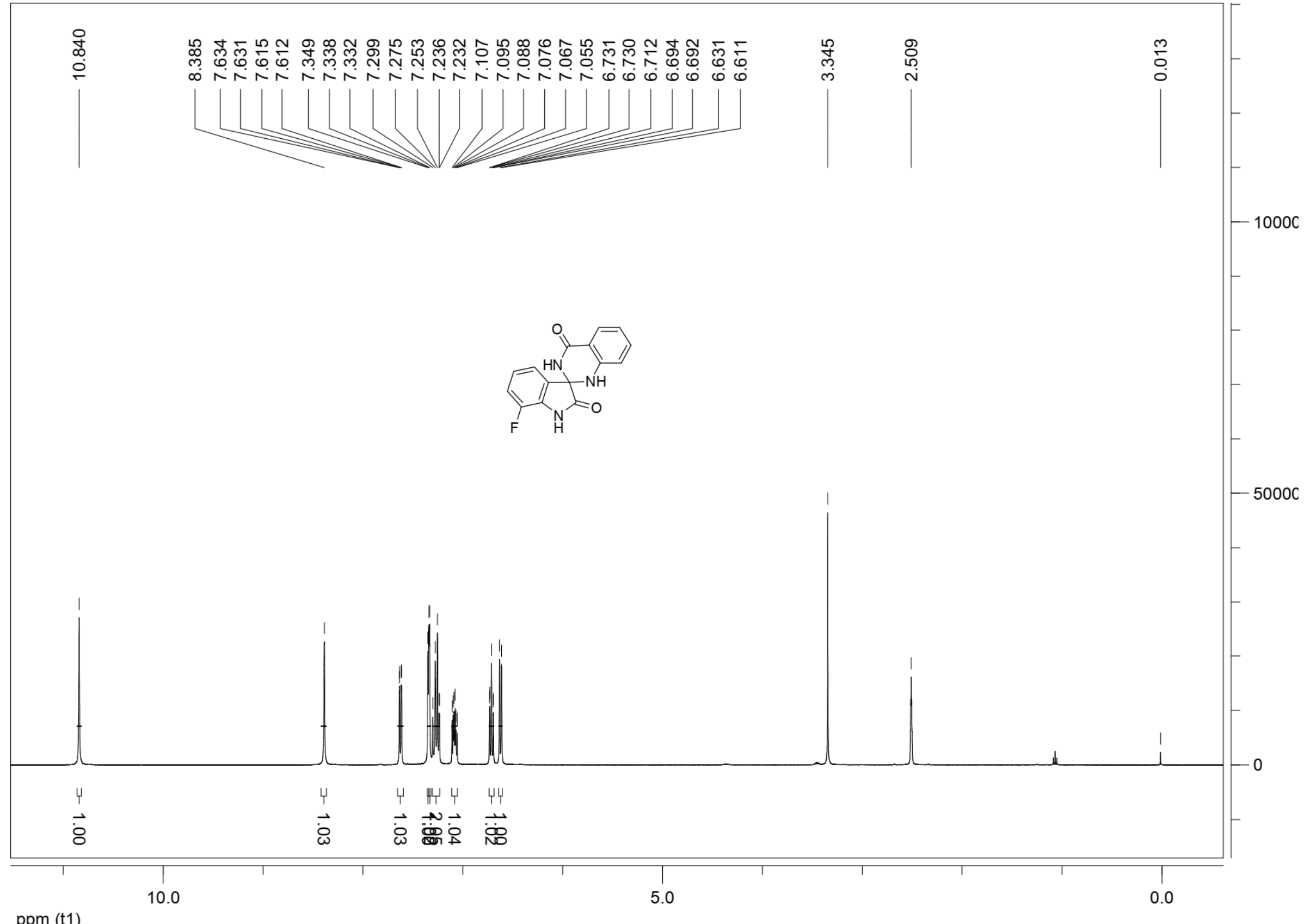

ppm (t1) 


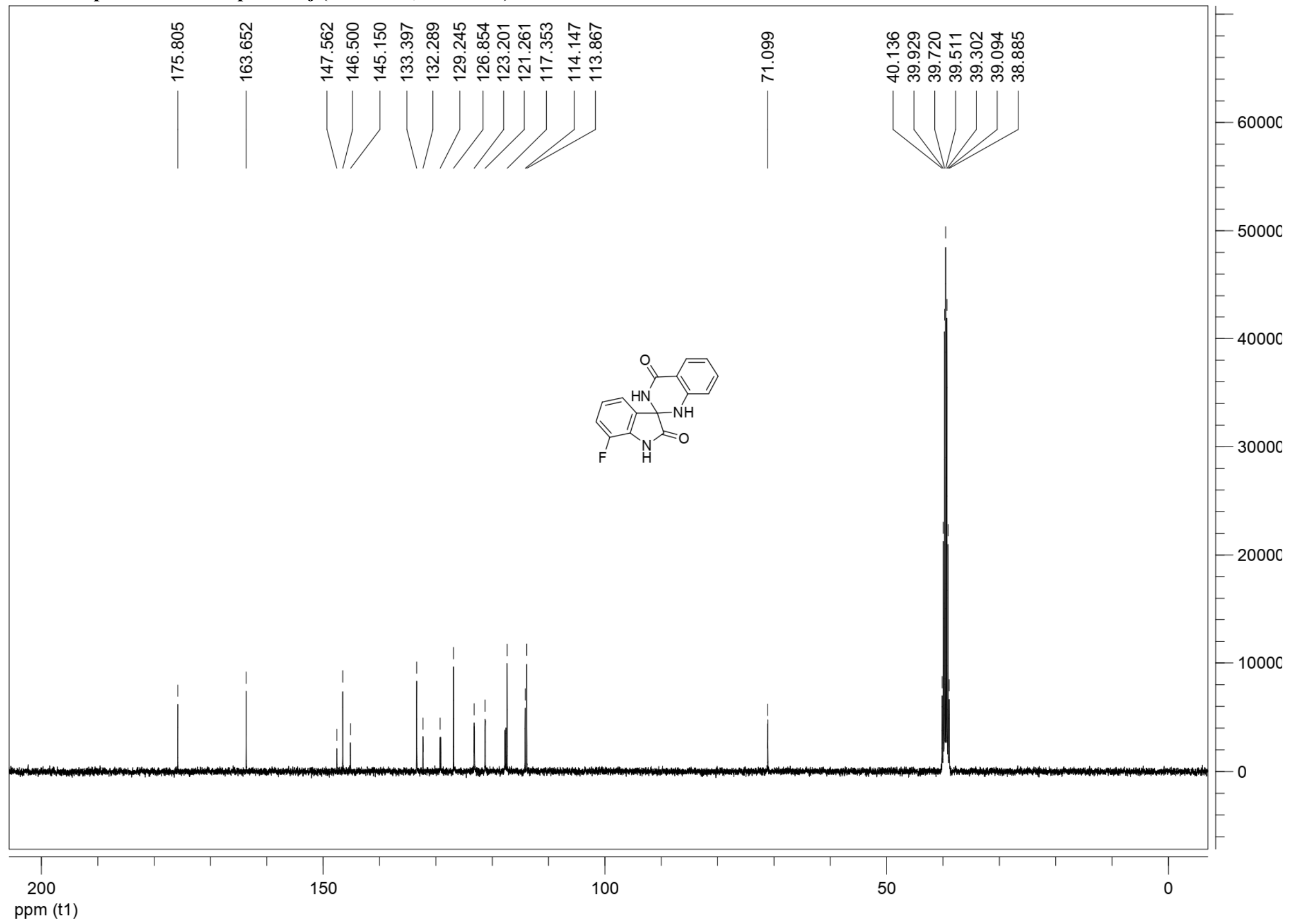


${ }^{1} \mathrm{H}$ NMR spectrum of Compound 3k (DMSO- $d_{6}, 400 \mathrm{MHz}$ )

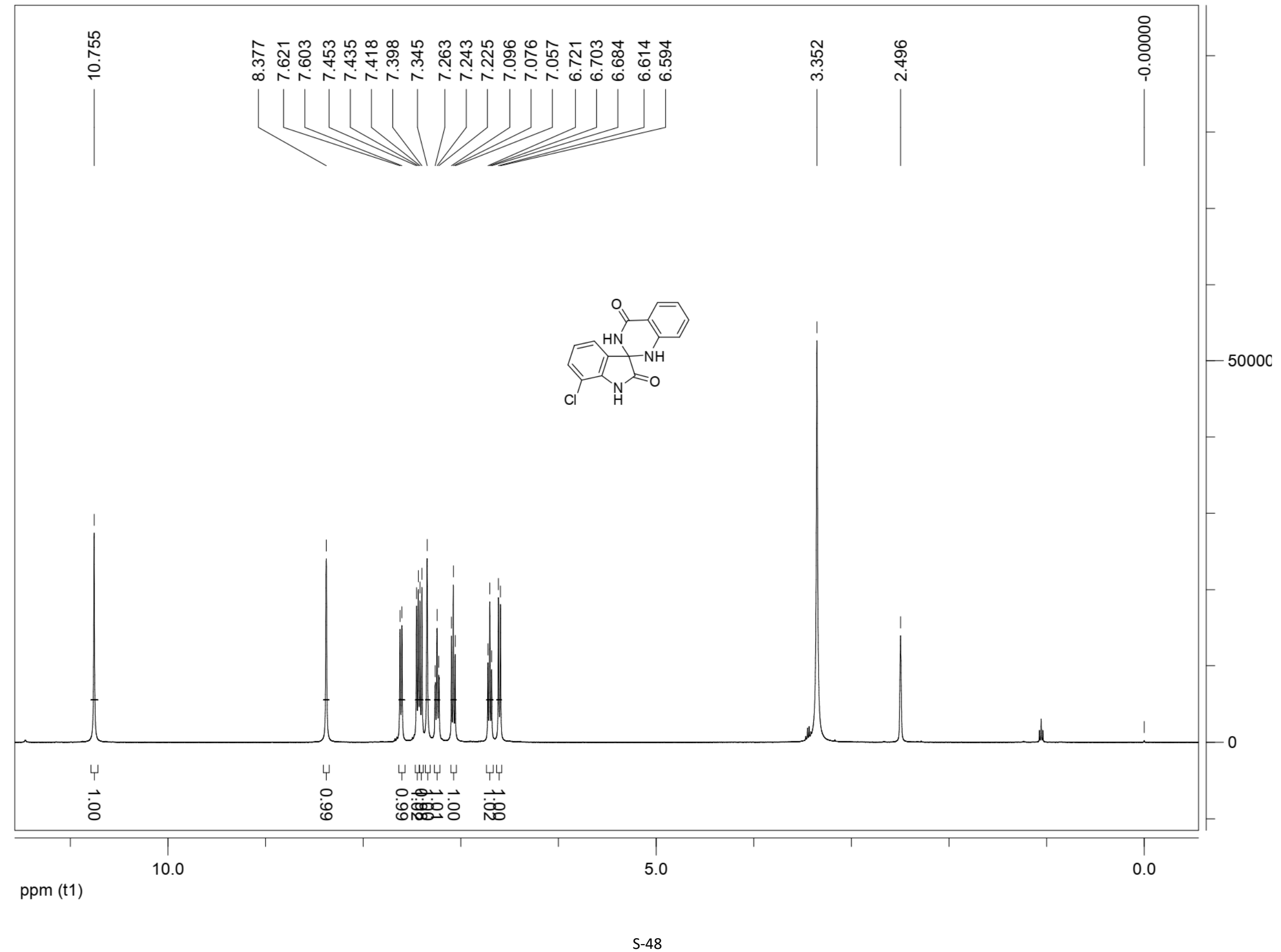




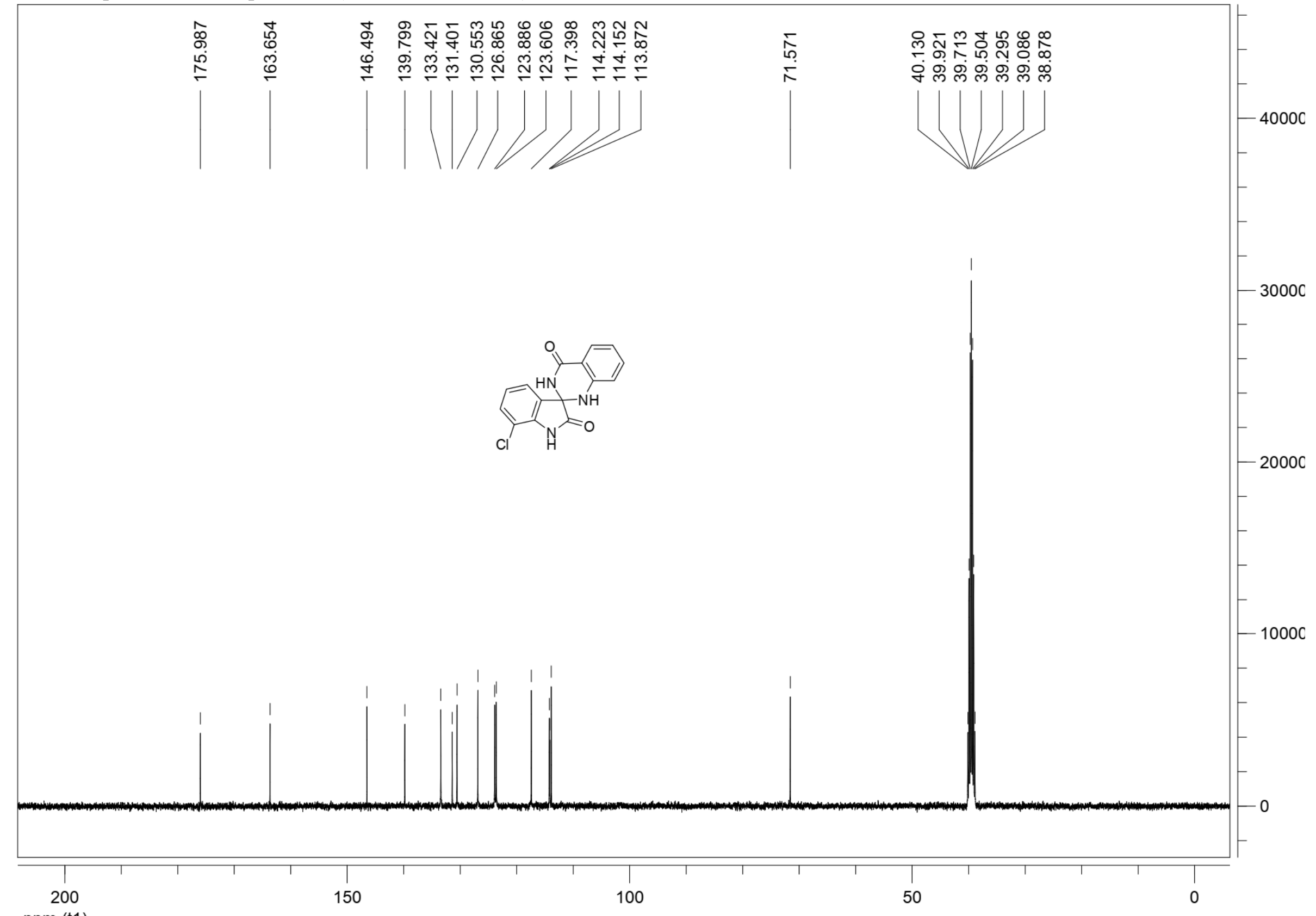




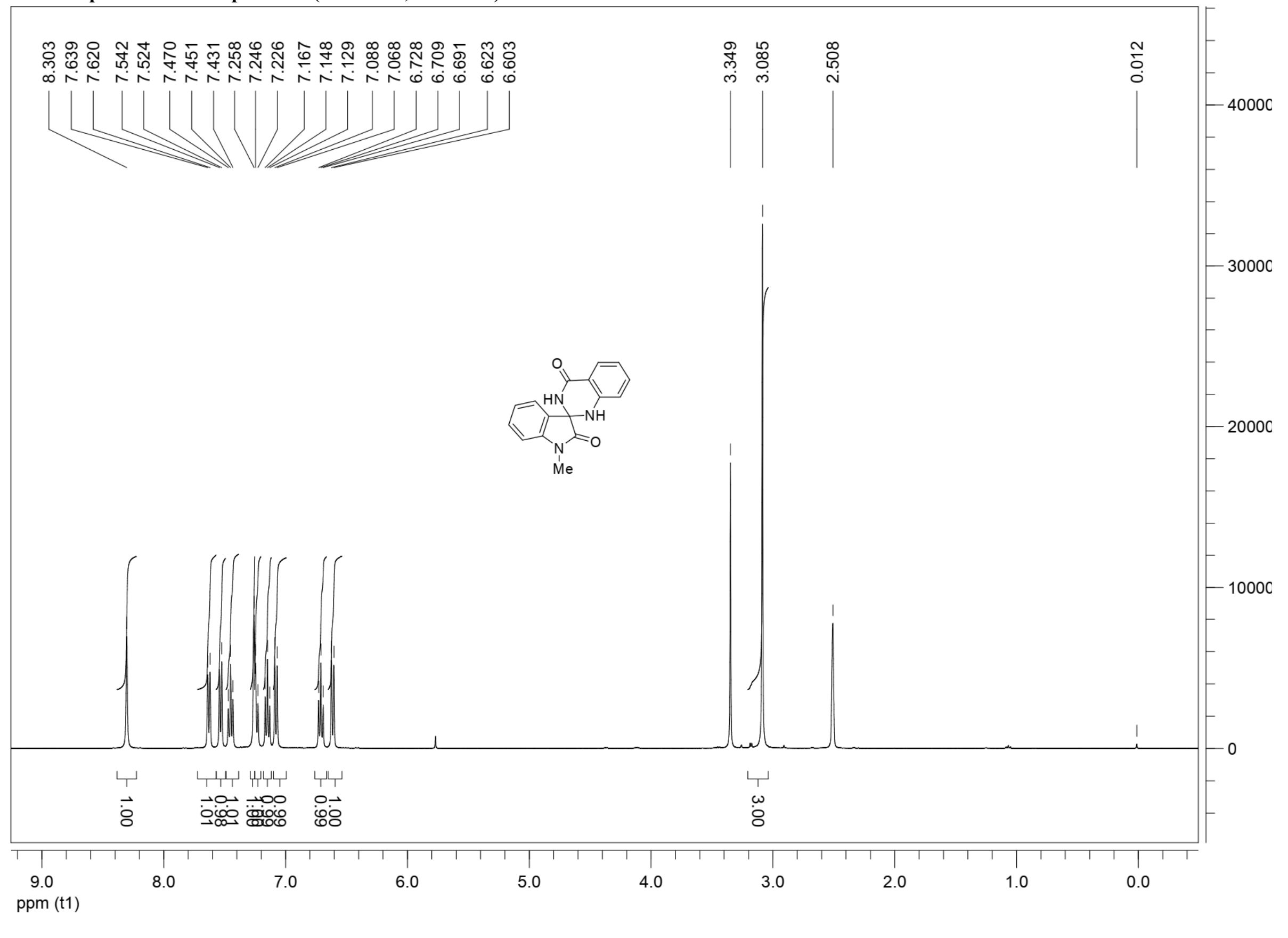




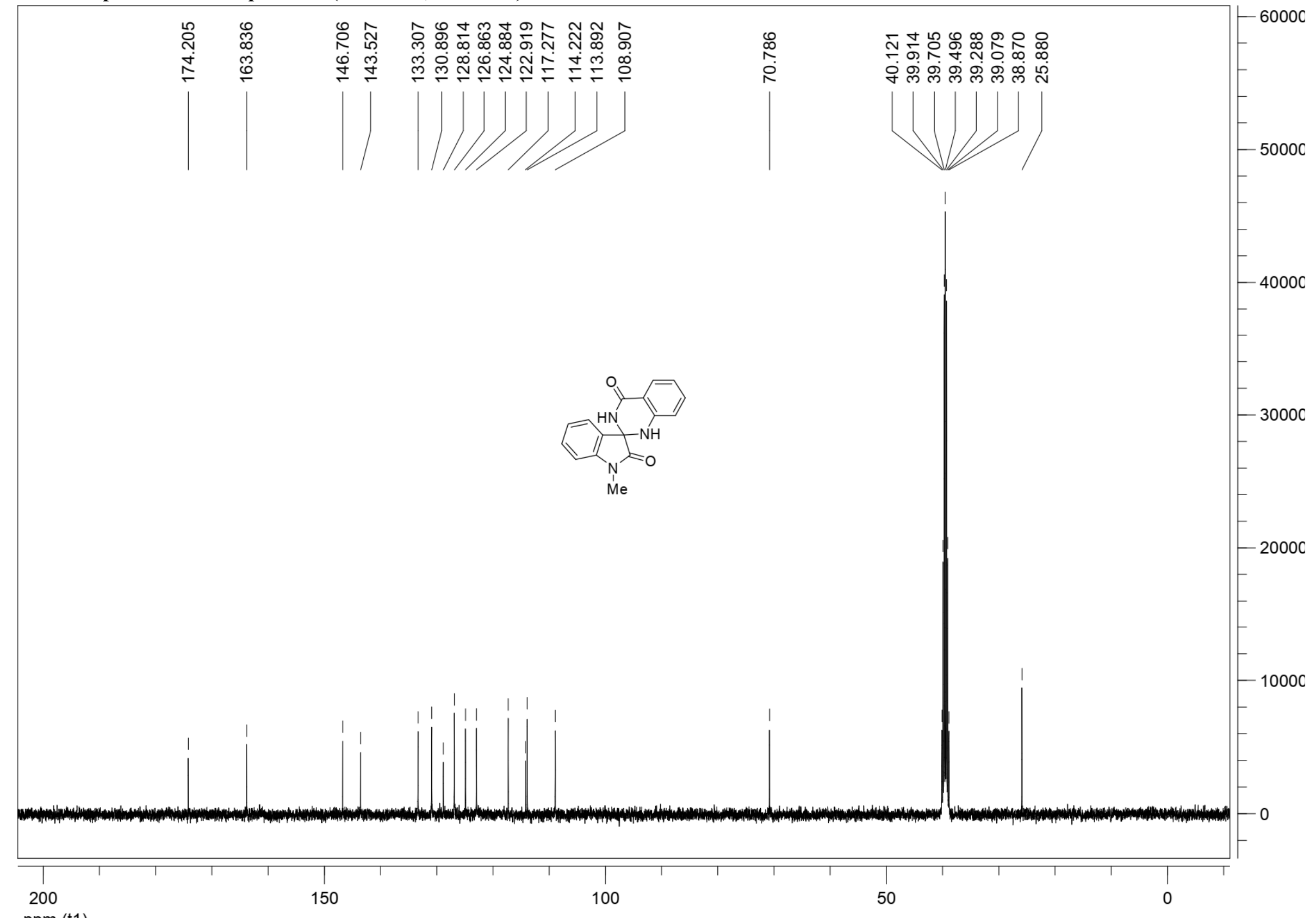

ppm (t1) 


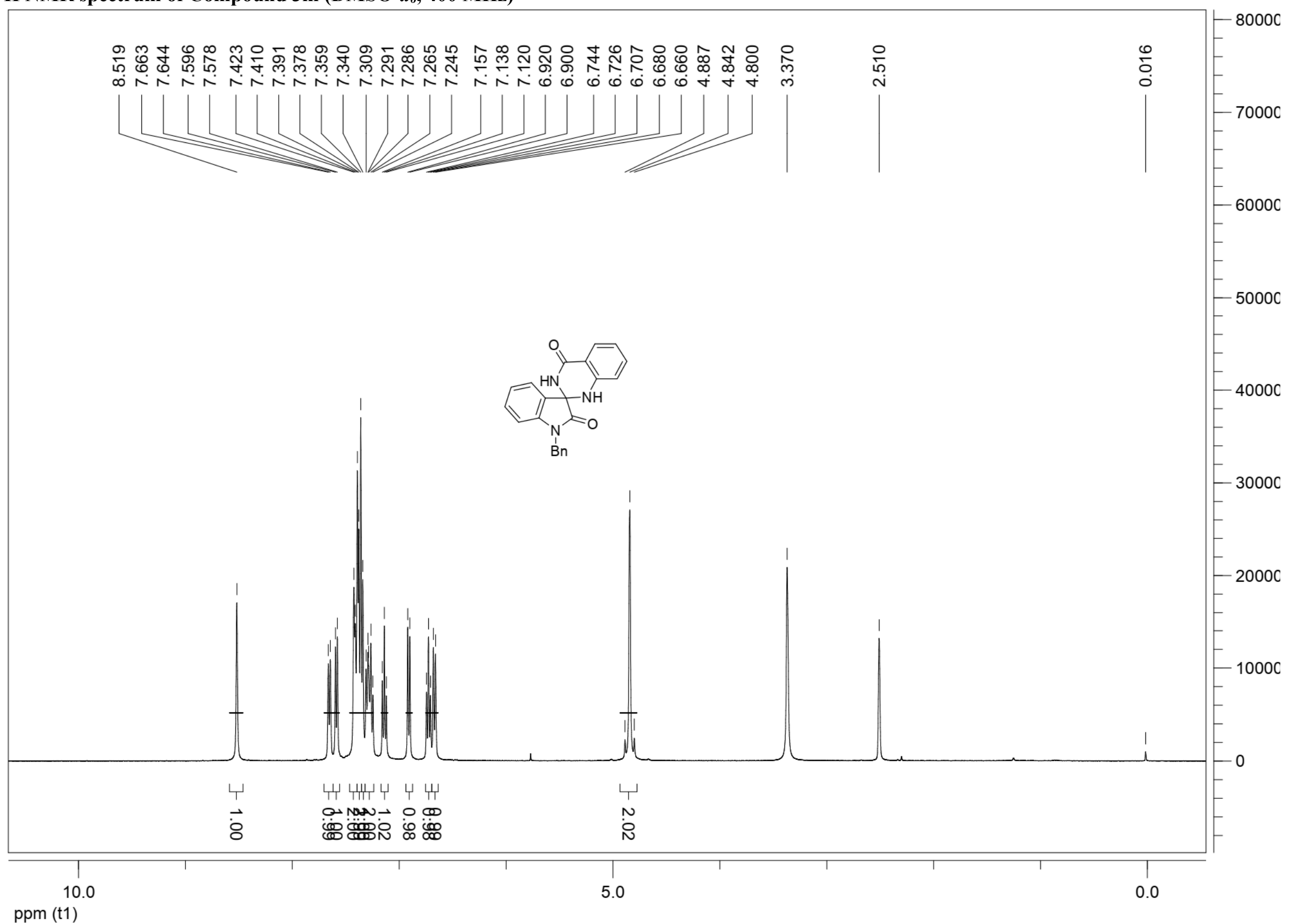




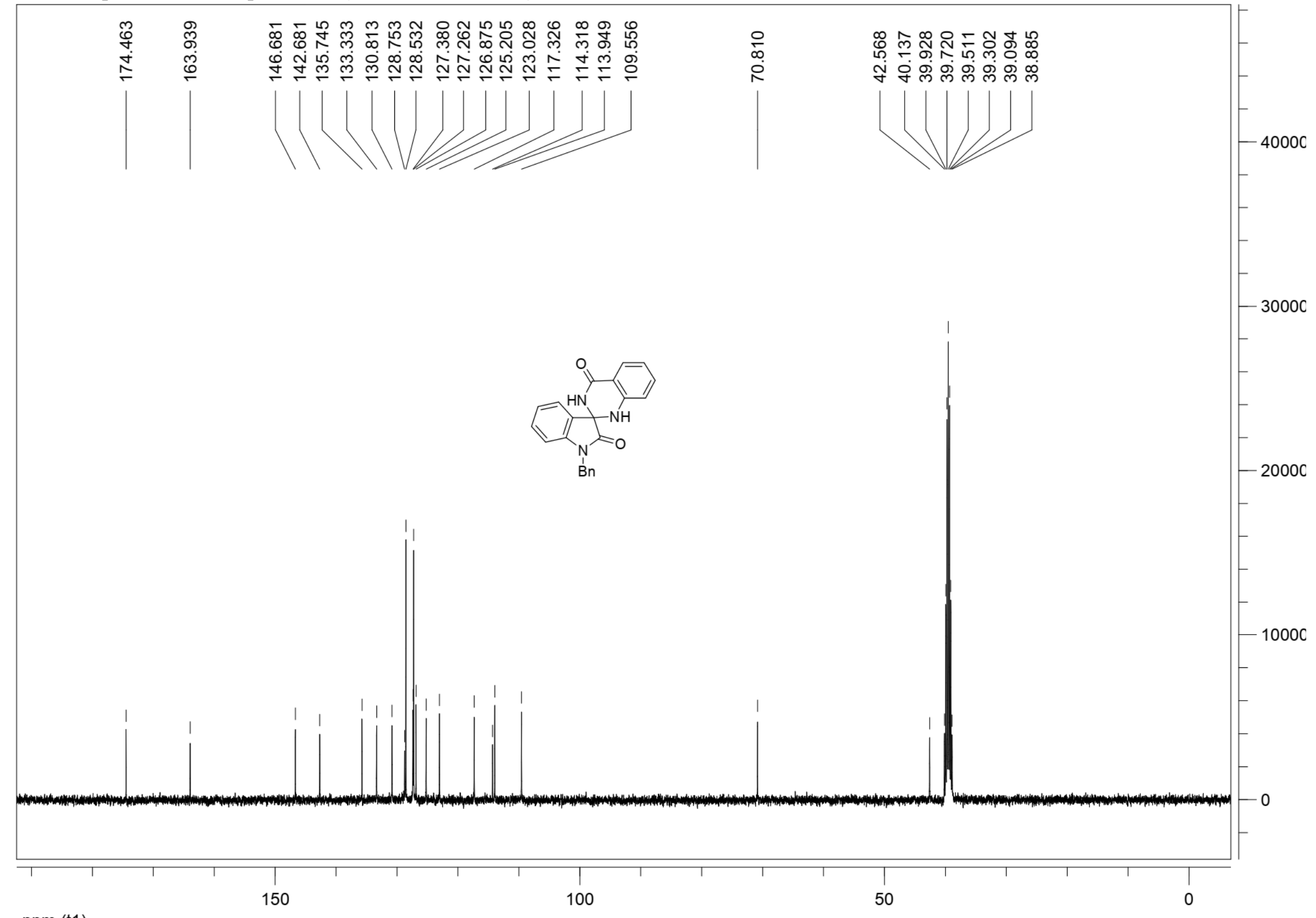

ppm (t1) 


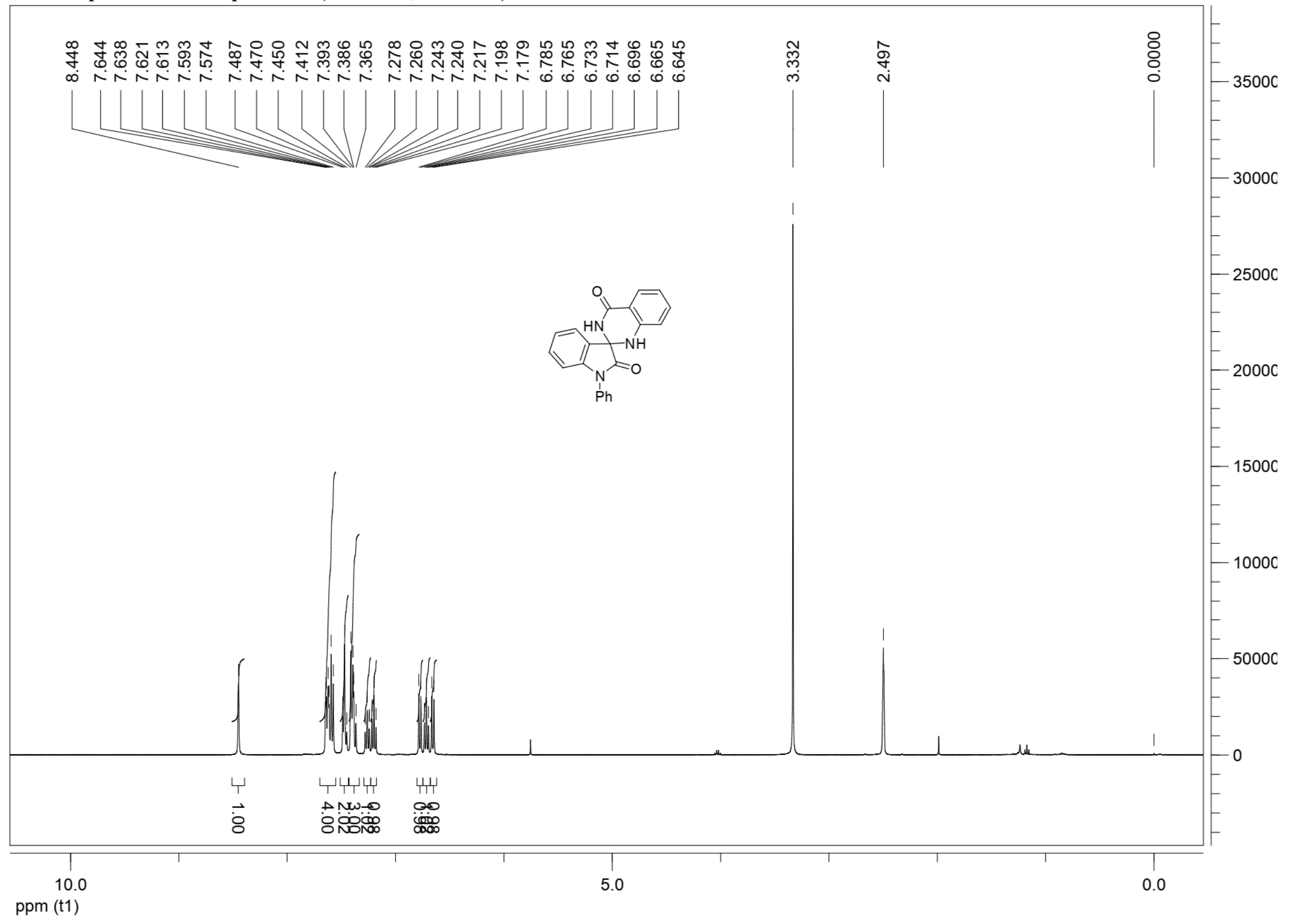


${ }^{13} \mathrm{C}$ NMR spectrum of Compound 3n (DMSO- $d_{6}, 100 \mathrm{MHz}$ )

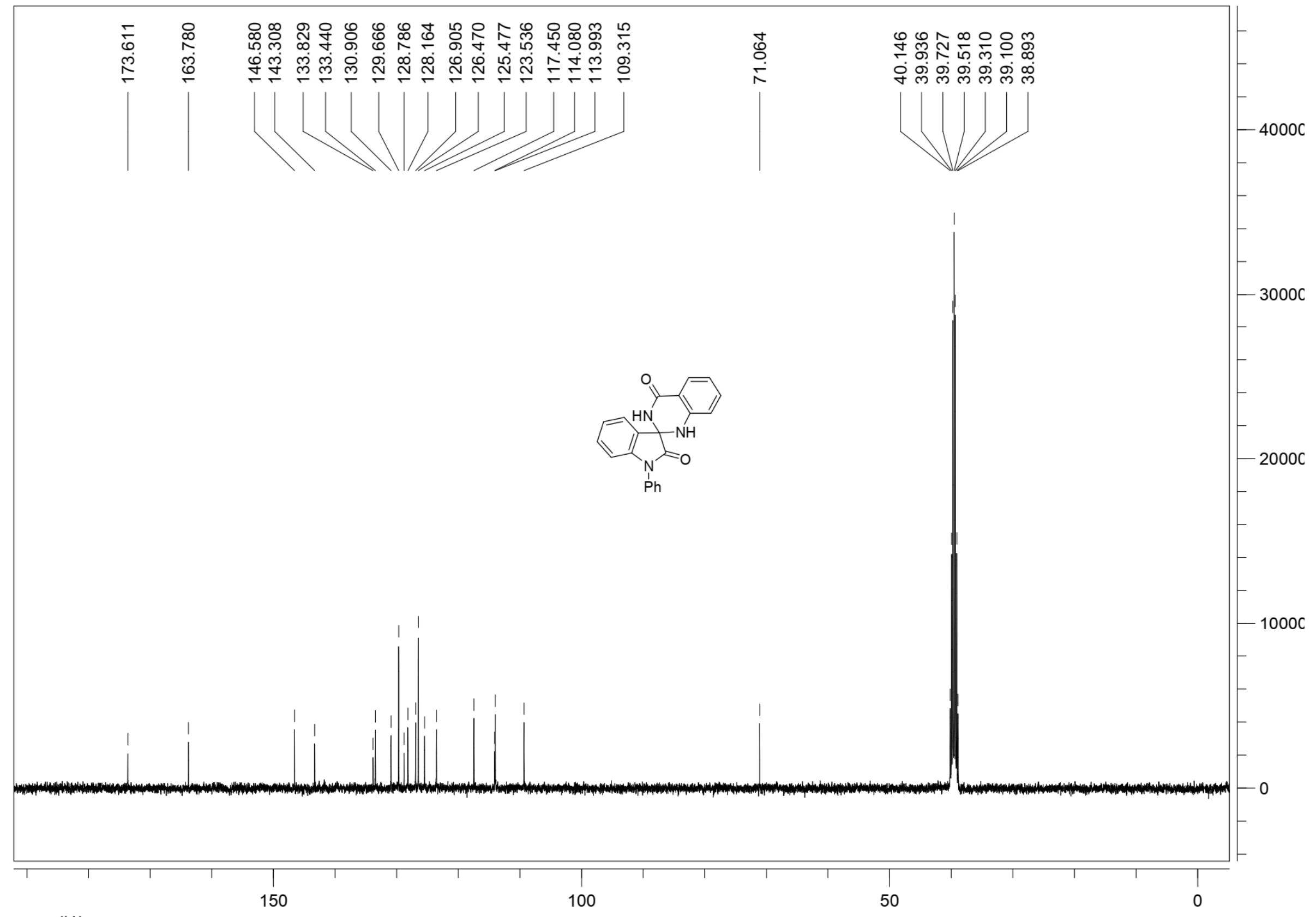

ppm (t1) 


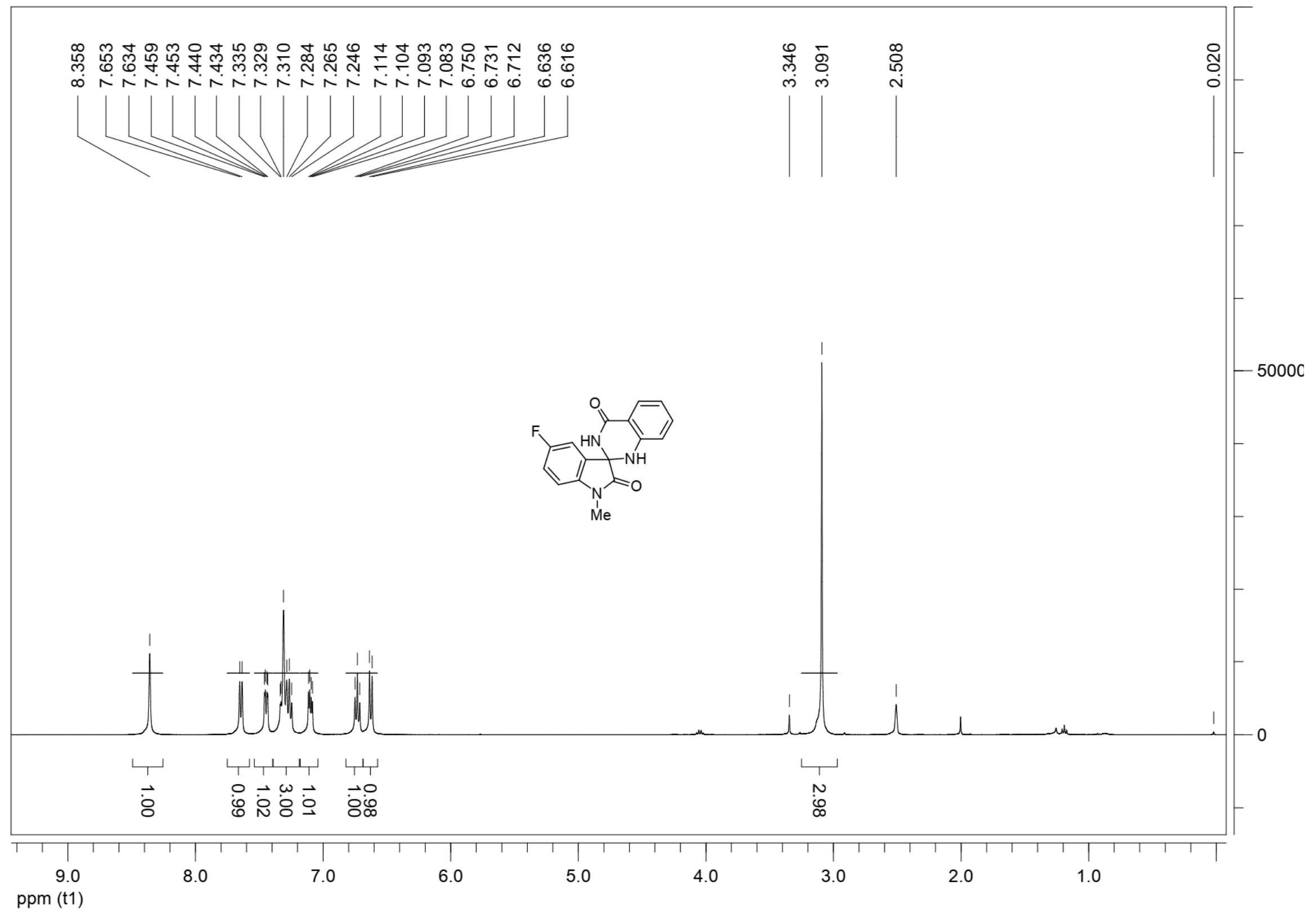


${ }^{13} \mathrm{C}$ NMR spectrum of Compound 30 (DMSO- $d_{6}, 100 \mathrm{MHz}$ )

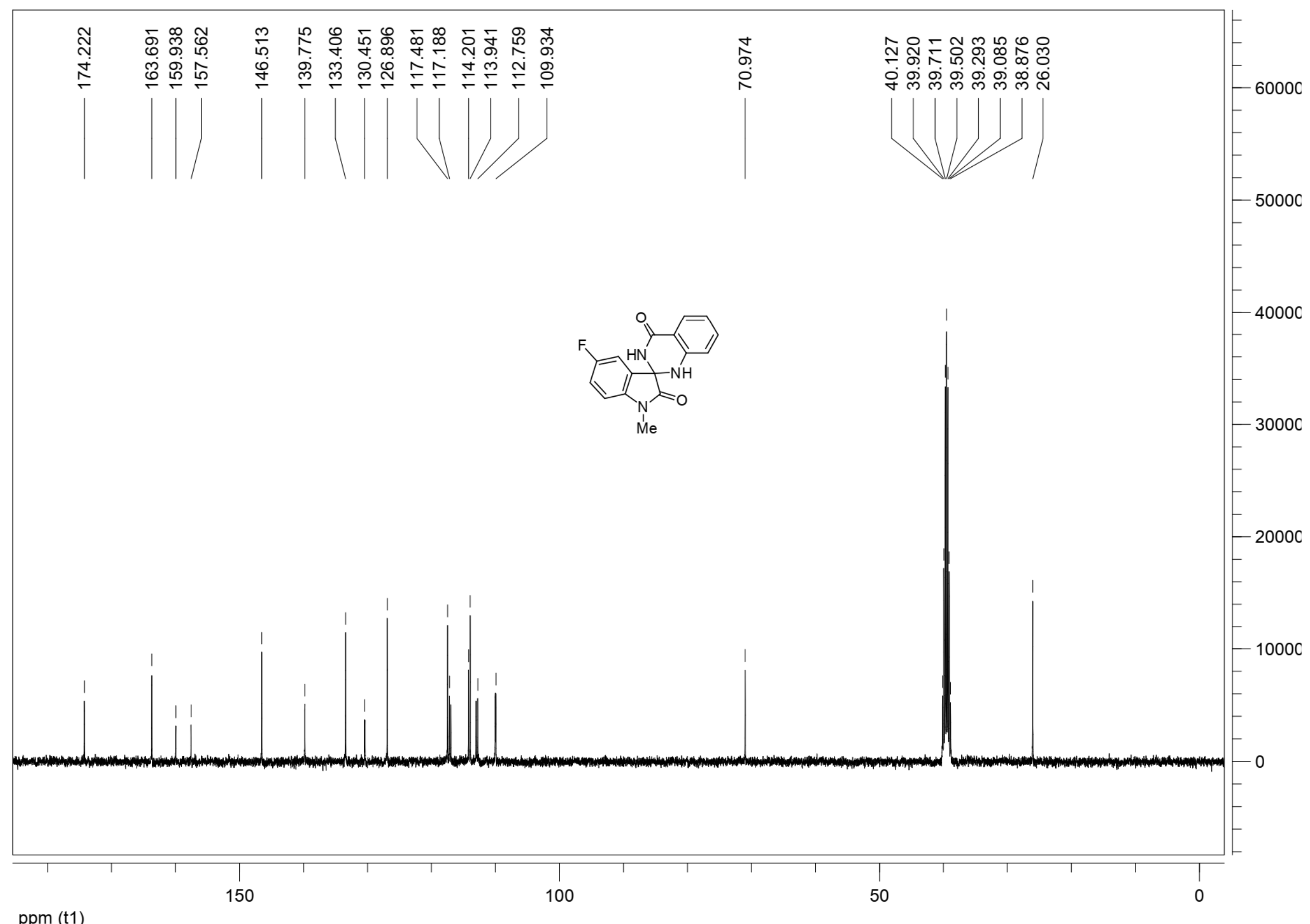

ppm (t1) 


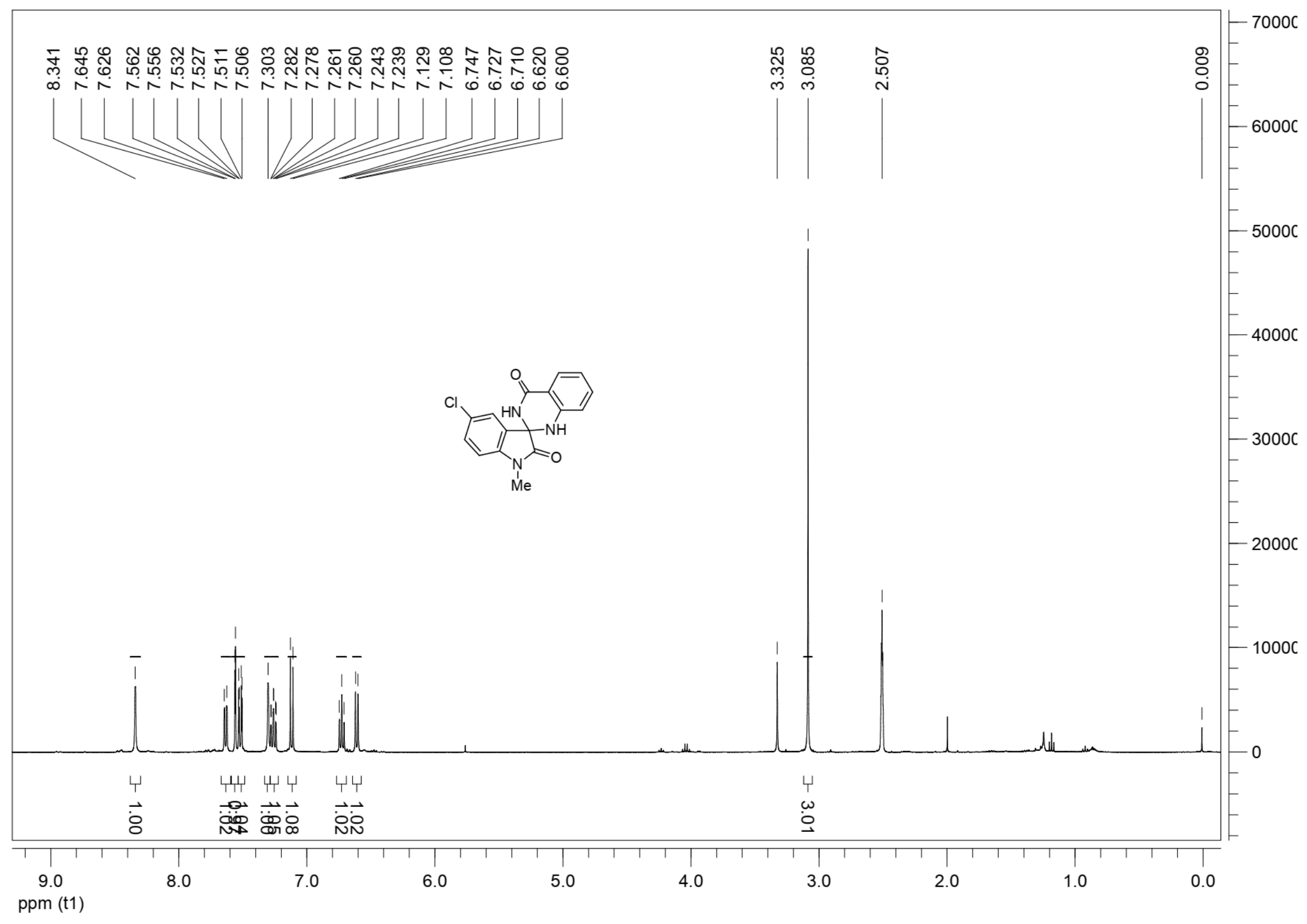


${ }^{13} \mathrm{C}$ NMR spectrum of Compound 3p (DMSO- $d_{6}, 100 \mathrm{MHz}$ )

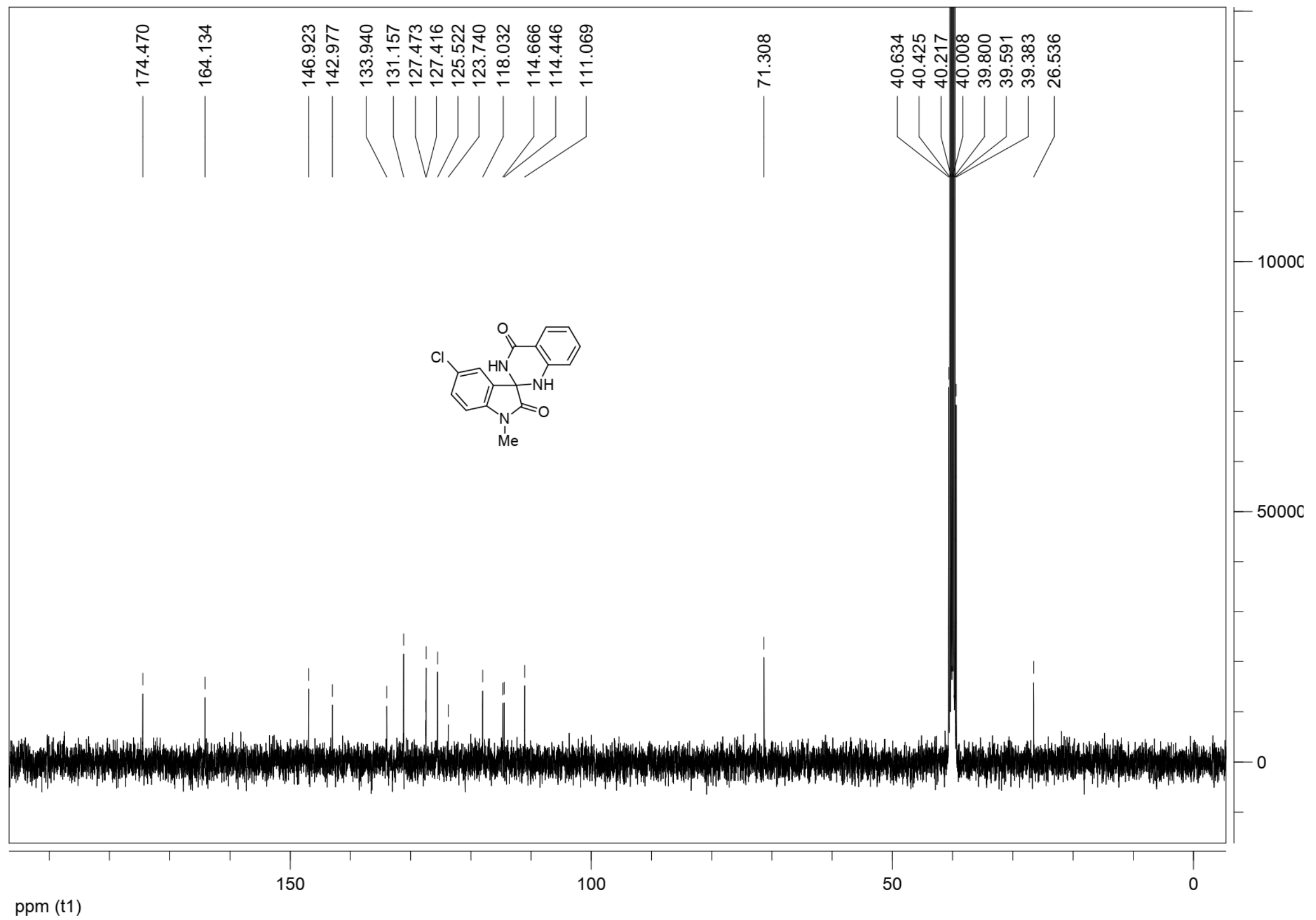


${ }^{1} \mathrm{H}$ NMR spectrum of Compound 3q (DMSO- $d_{6}, 400 \mathrm{MHz}$ )

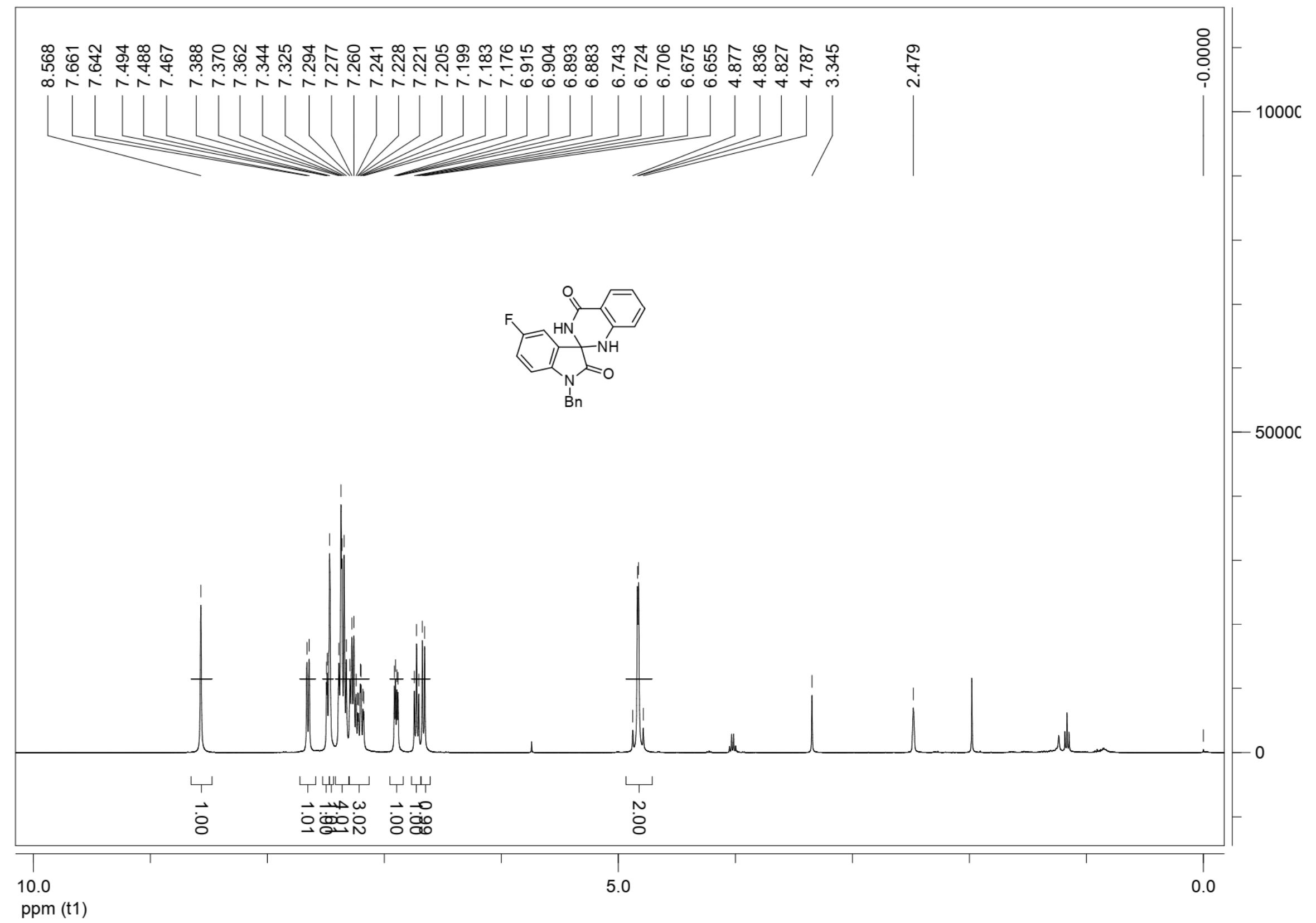




\section{${ }^{13} \mathrm{C}$ NMR spectrum of Compound 3q (DMSO- $d_{6}, 100 \mathrm{MHz}$ )}

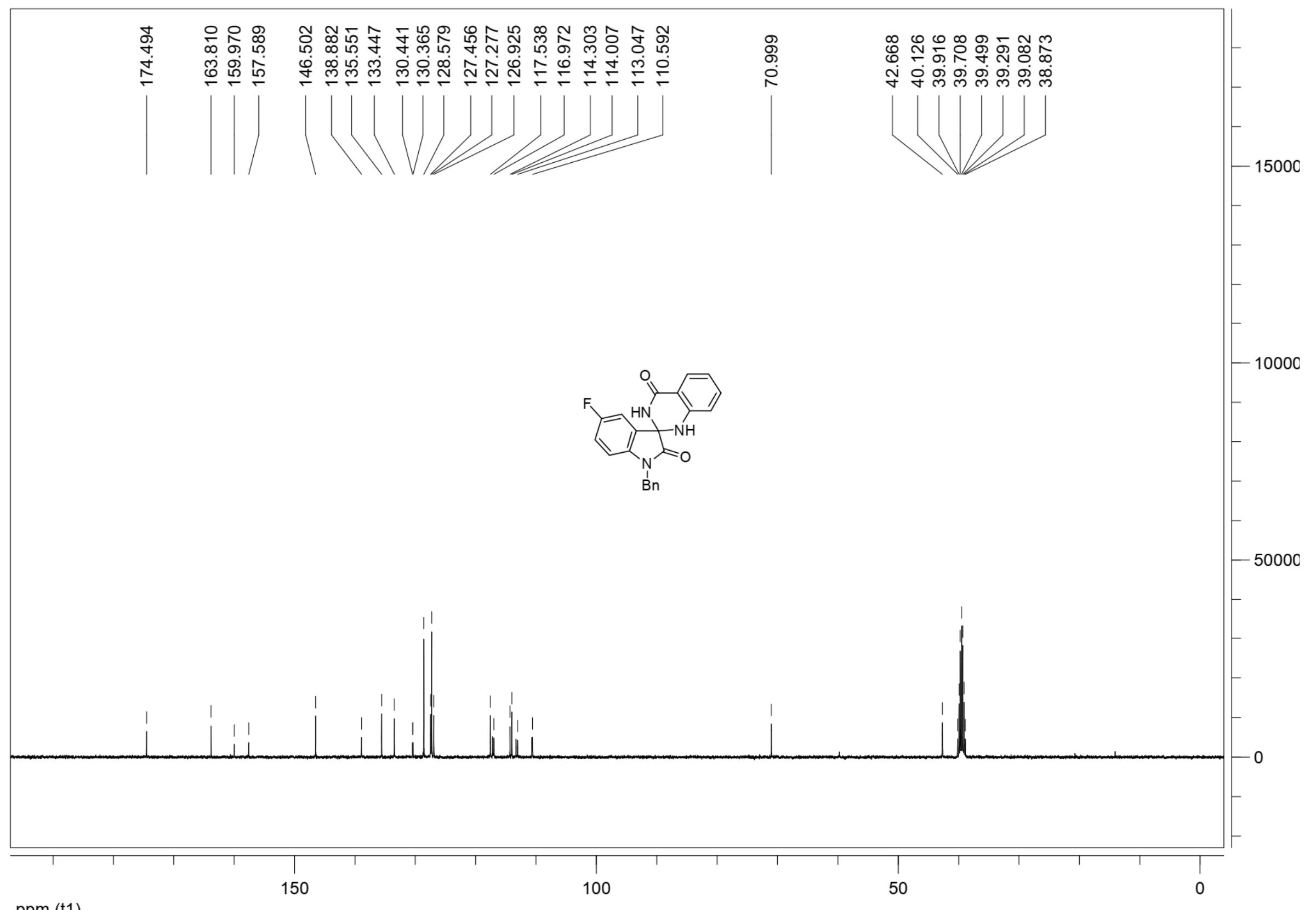

ppm (t1) 


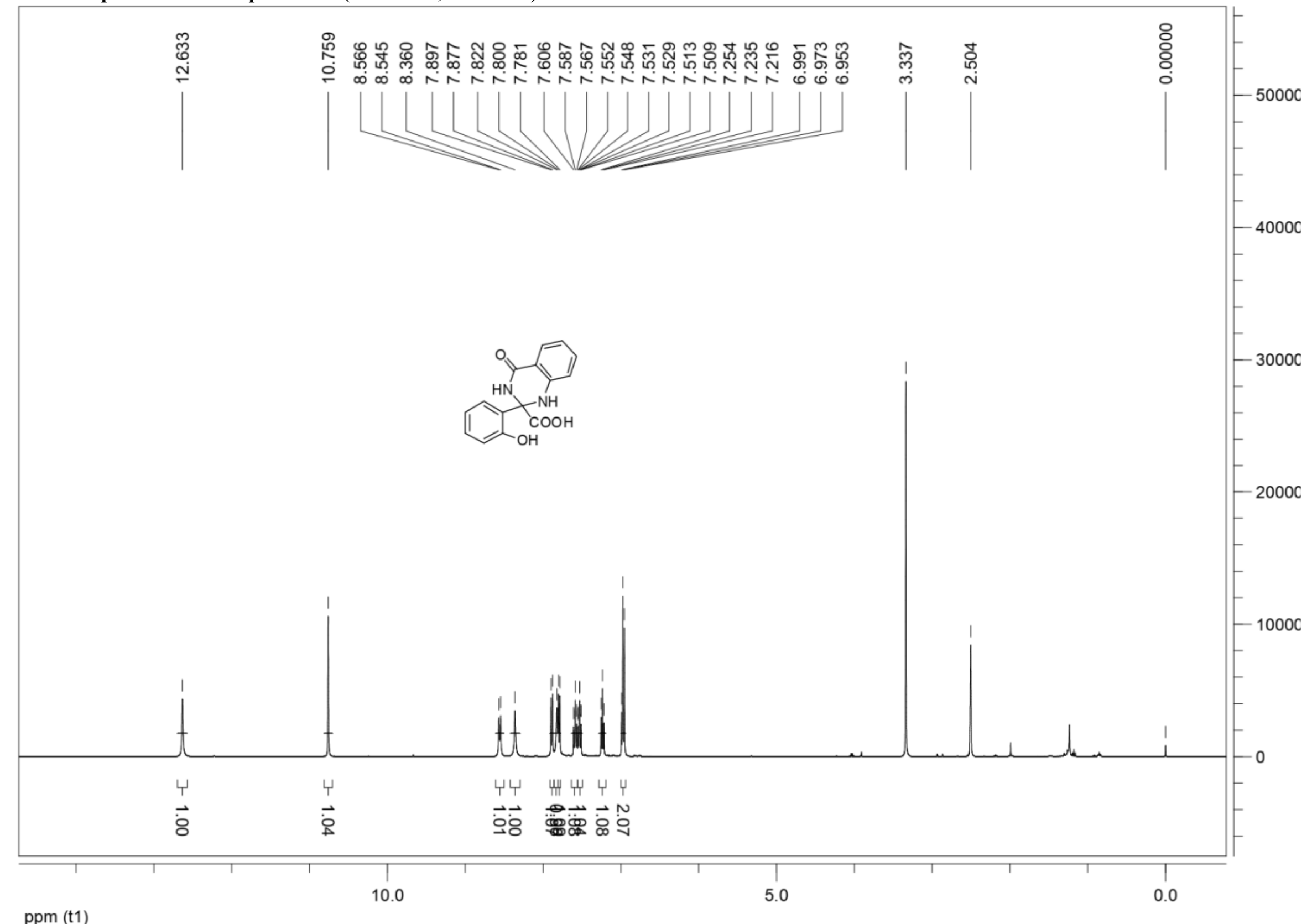


${ }^{13} \mathrm{C}$ NMR spectrum of Compound $3 r^{\prime}$ (DMSO- $d_{6}, 100 \mathrm{MHz}$ )

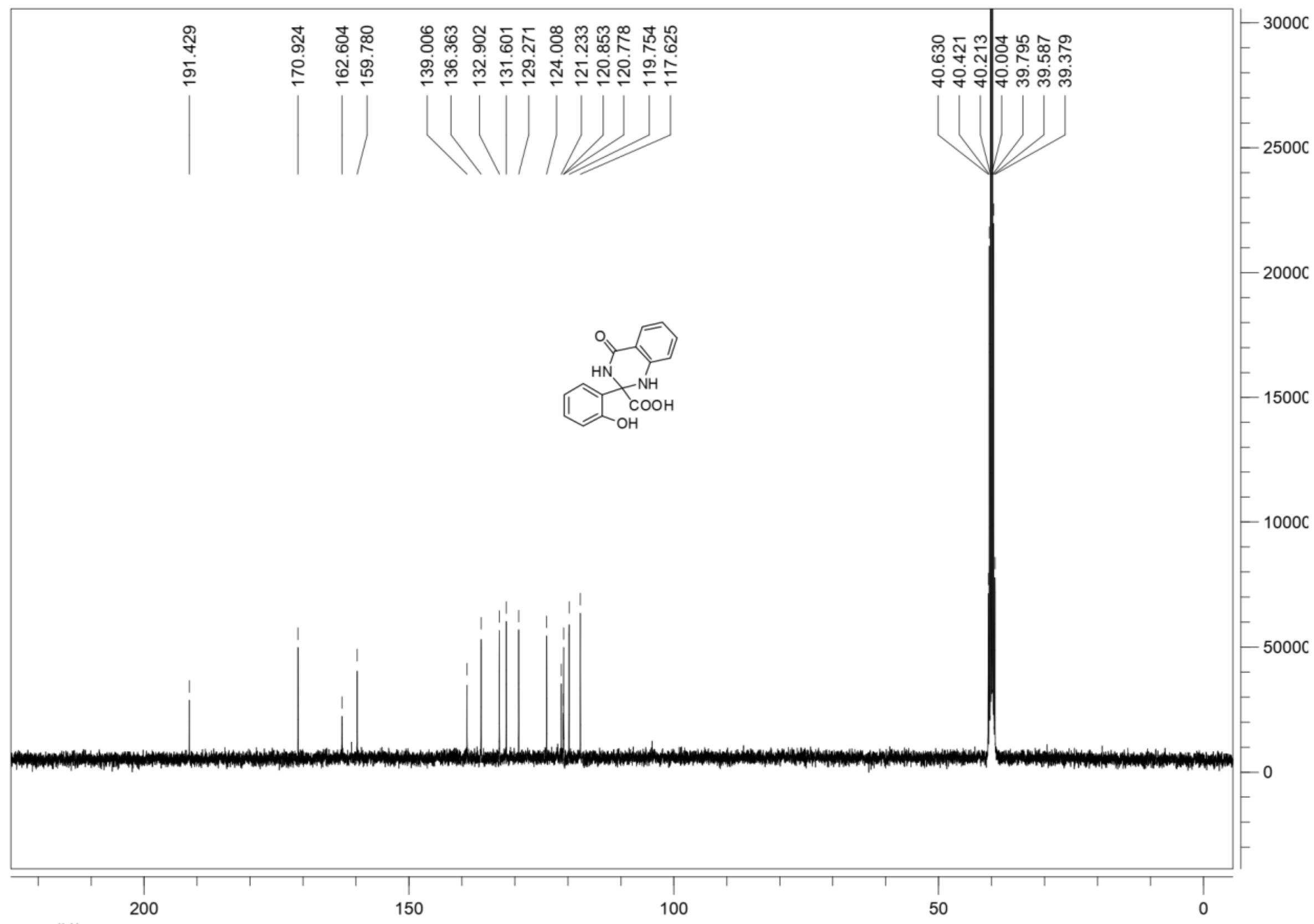

ppm (t1) 
${ }^{1} \mathrm{H}$ NMR spectrum of Compound 3s (DMSO- $d_{6}, 400 \mathrm{MHz}$ )

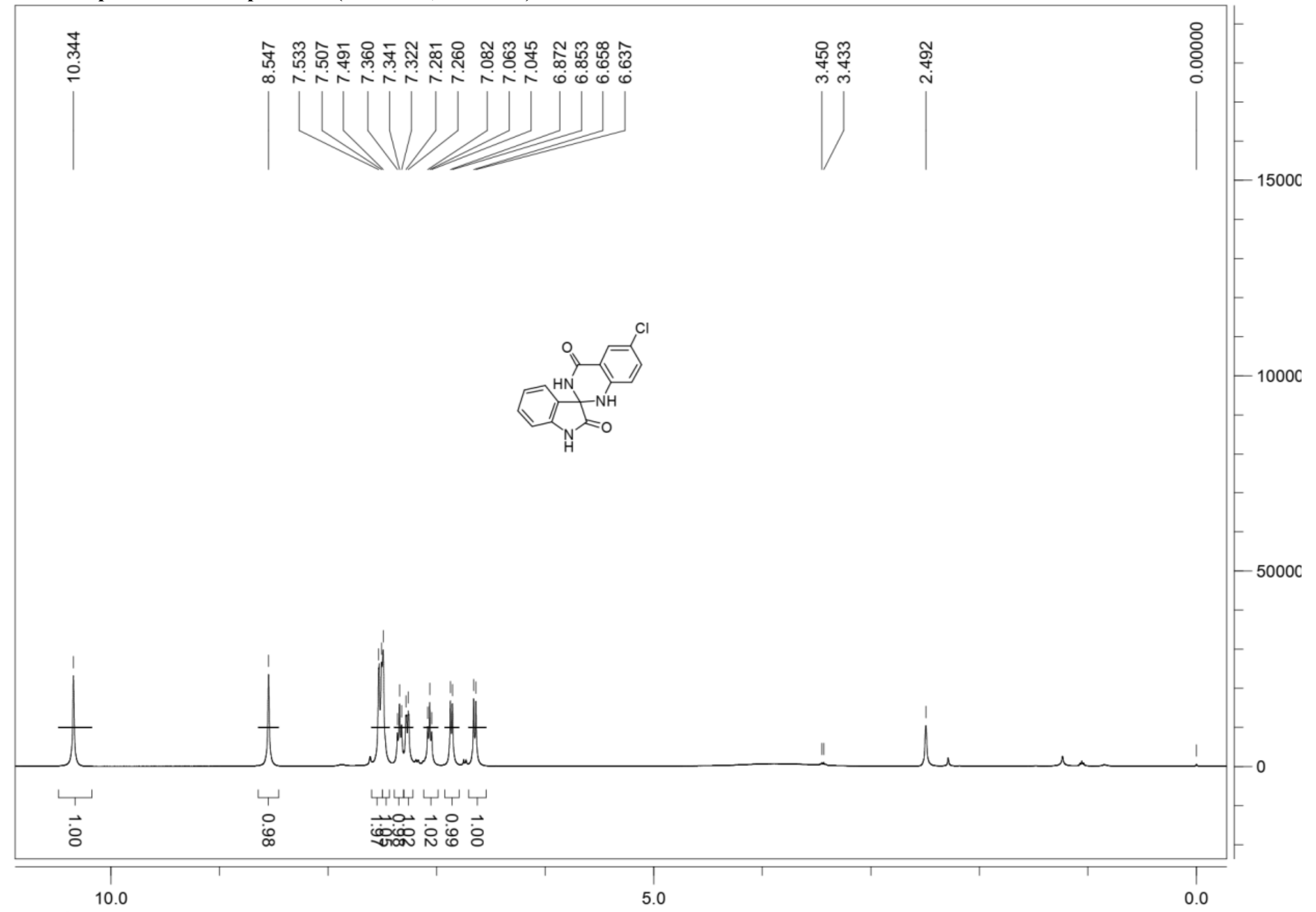

ppm (t1) 
${ }^{13} \mathrm{C}$ NMR spectrum of Compound 3s (DMSO- $d_{6}, 100 \mathrm{MHz}$ )

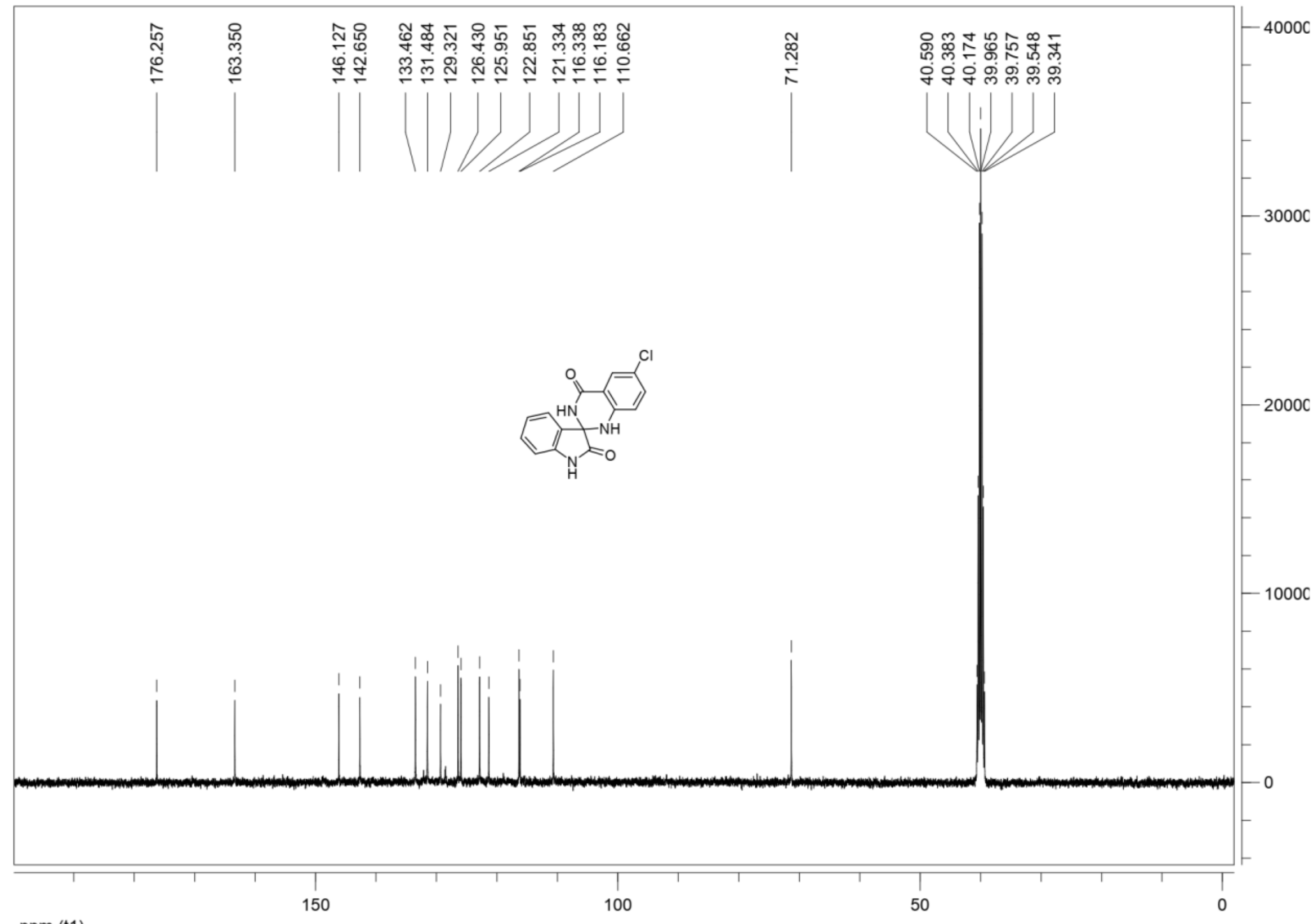

ppm (t1) 


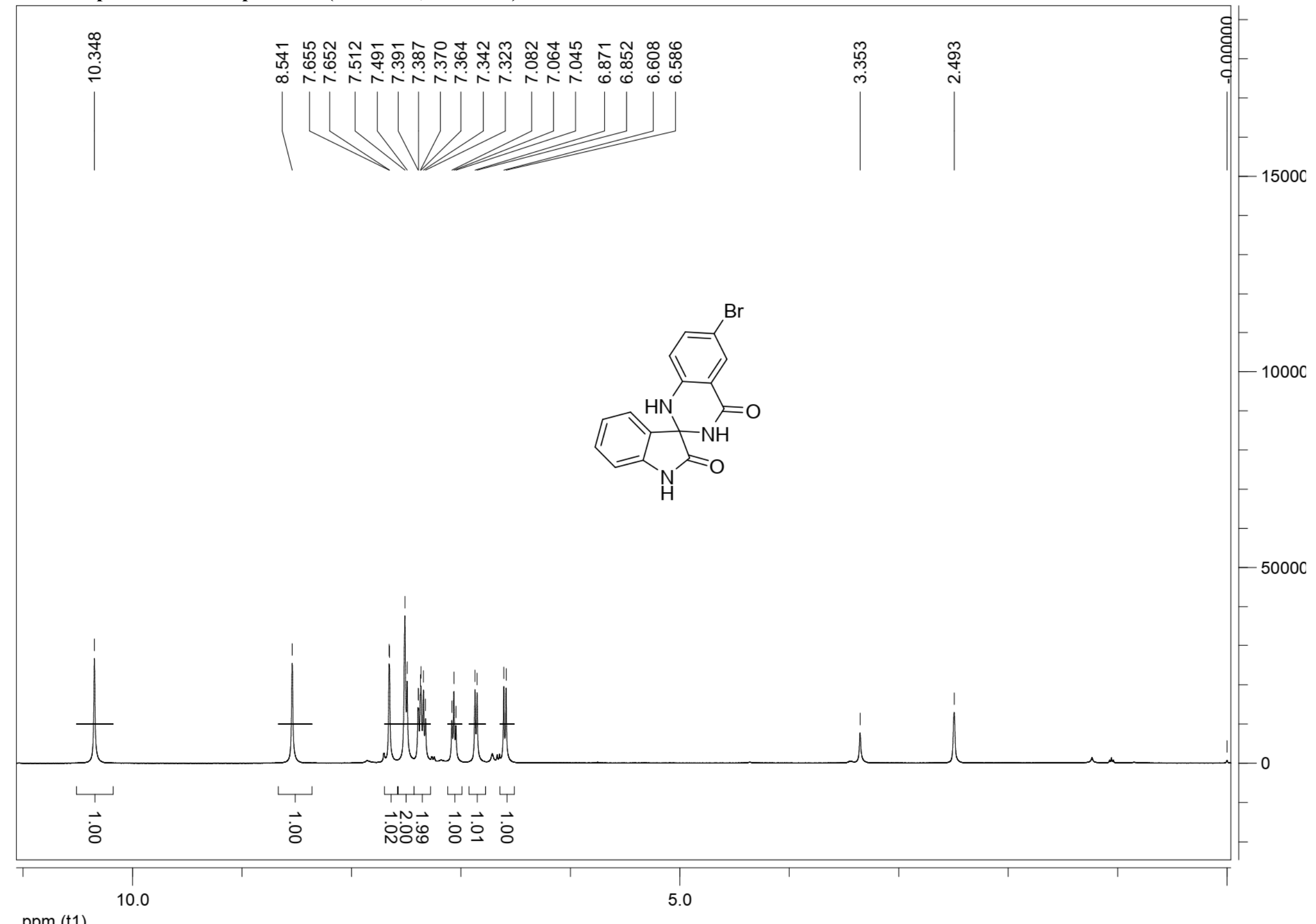


${ }^{13} \mathrm{C}$ NMR spectrum of Compound 3t (DMSO- $d_{6}, 100 \mathrm{MHz}$ )

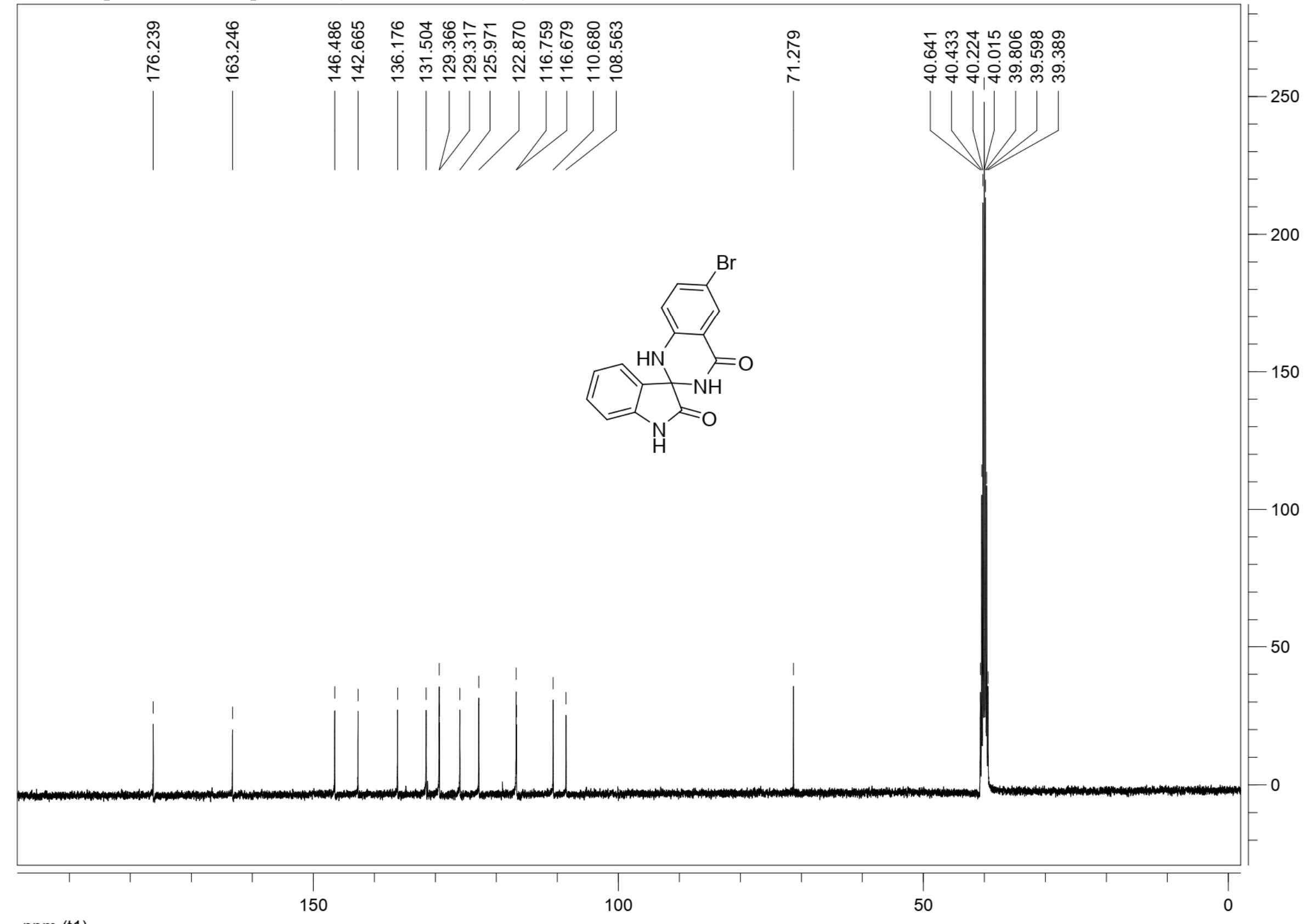

ppm (t1) 
${ }^{1} \mathrm{H}$ NMR spectrum of Compound 3u (DMSO- $\left.d_{6}, 400 \mathrm{MHz}\right)$

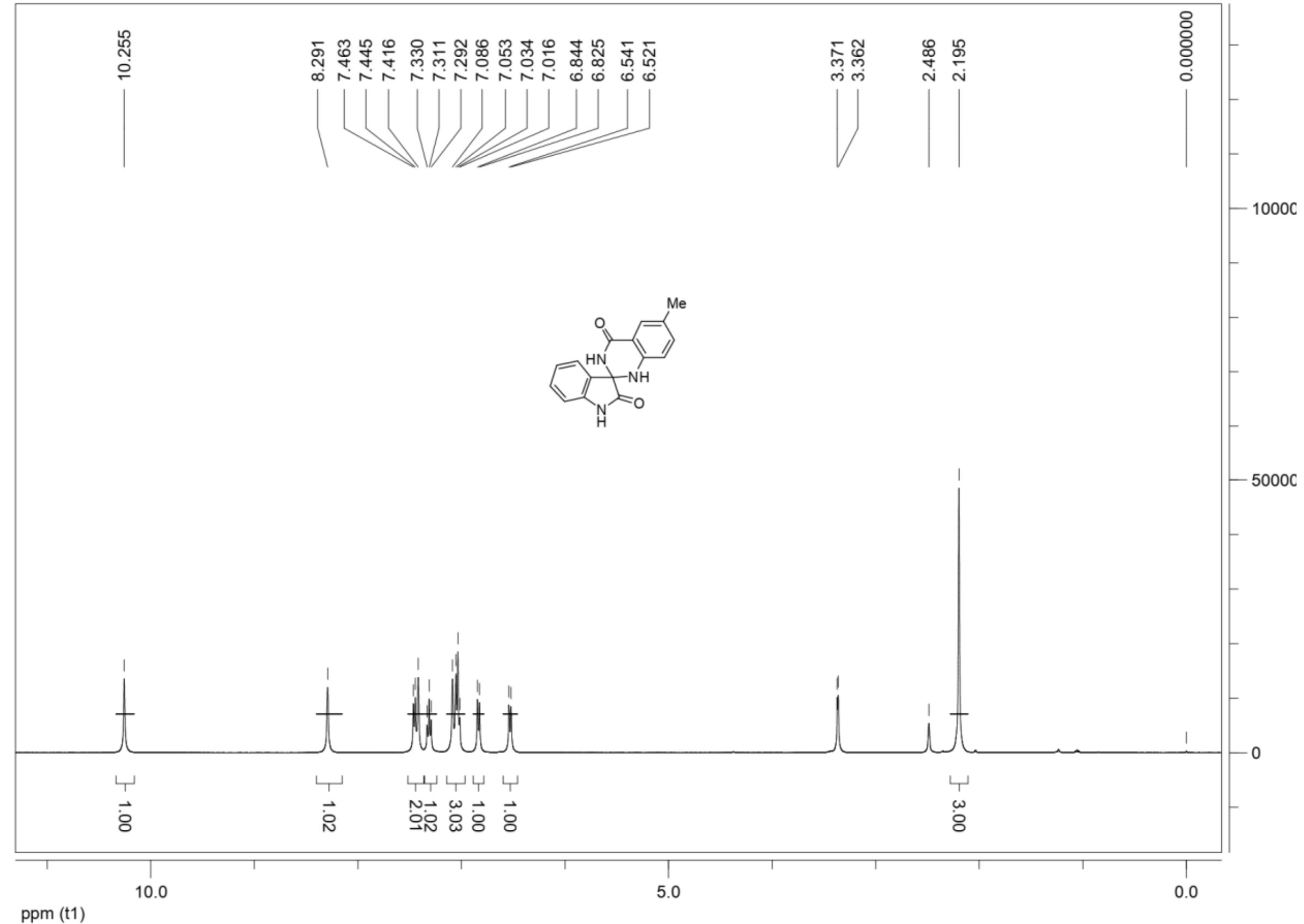




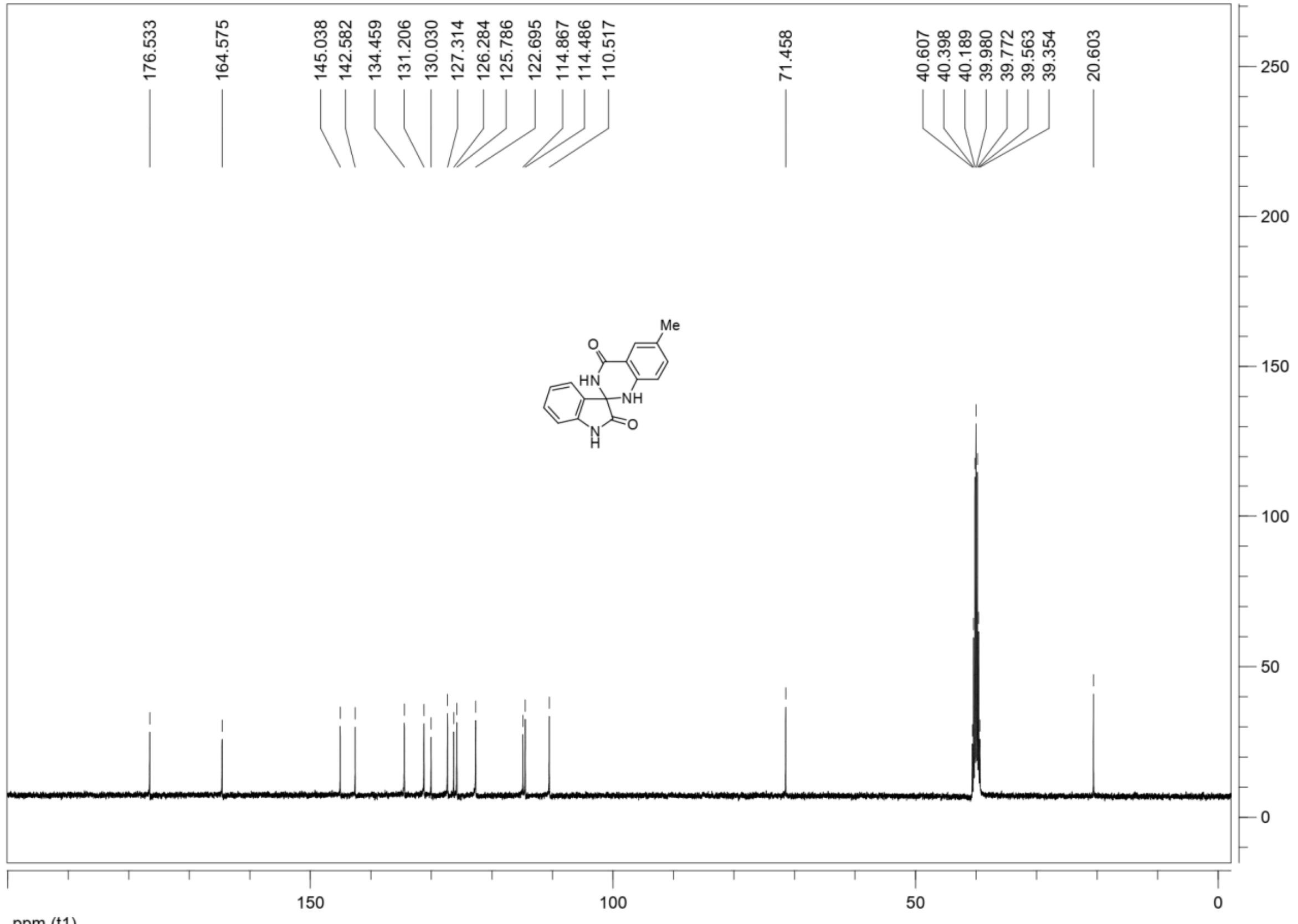




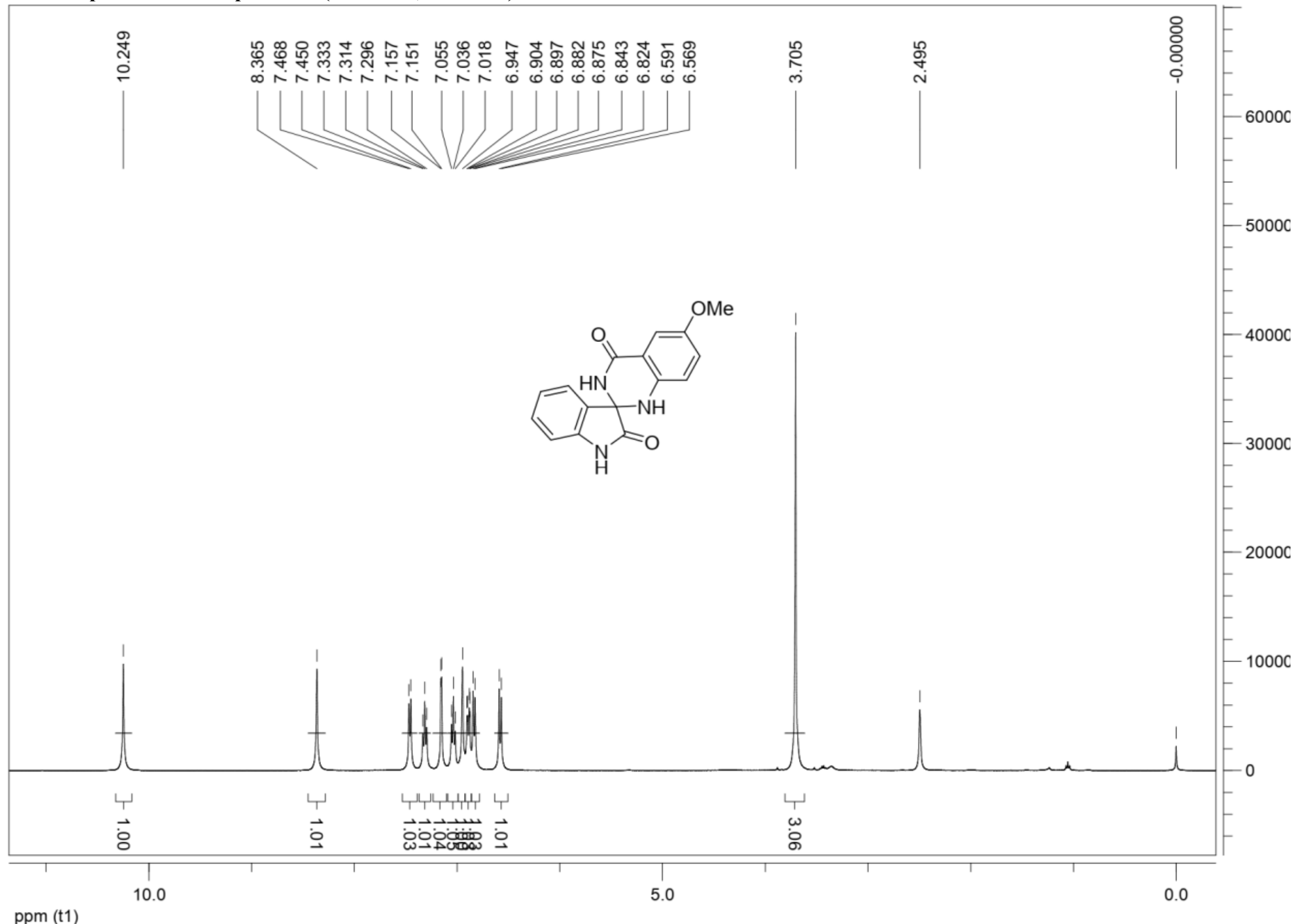




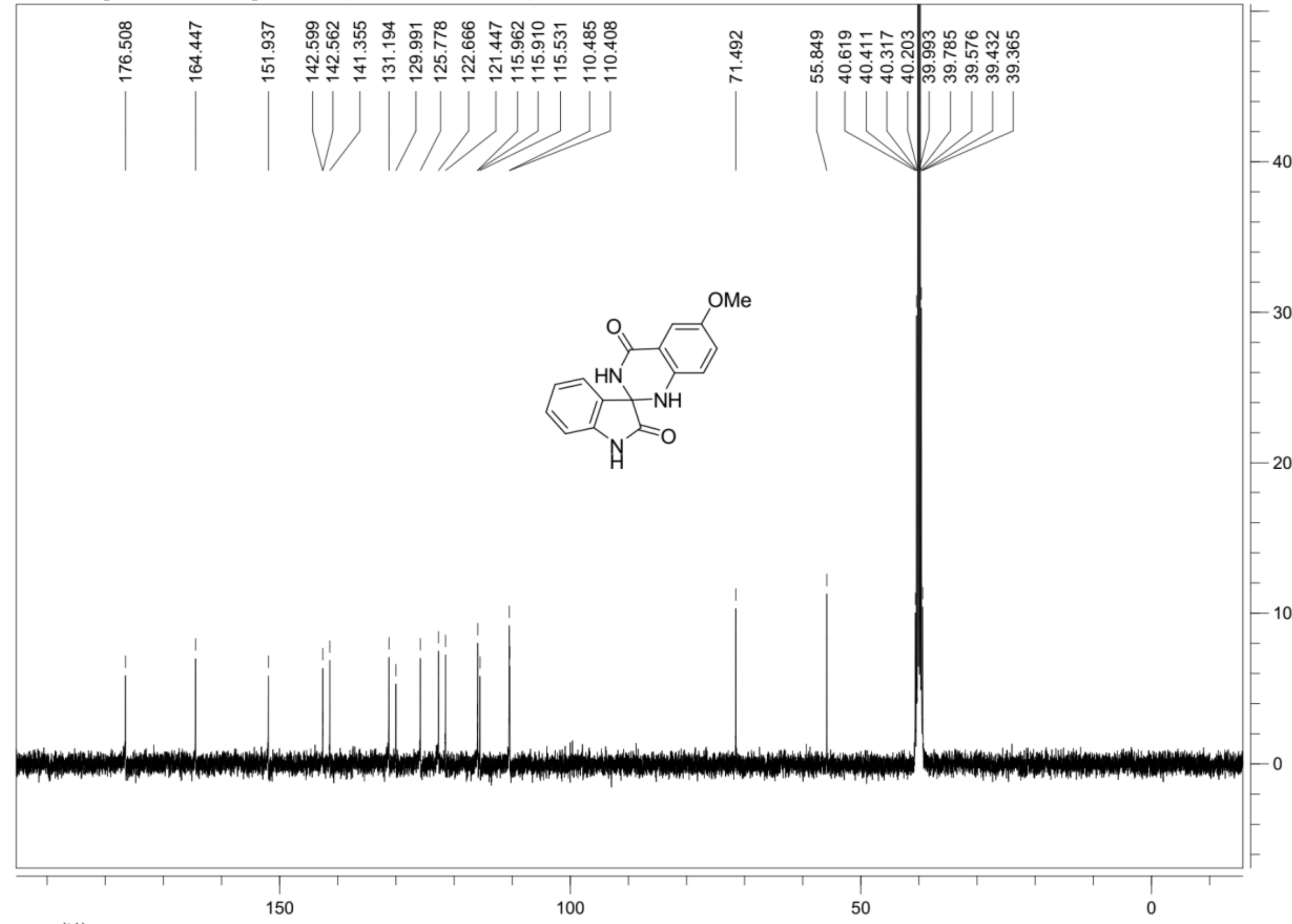

ppm (t1) 
${ }^{1} \mathrm{H}$ NMR spectrum of Compound 3w (DMSO- $d_{6}, 400 \mathrm{MHz}$ )

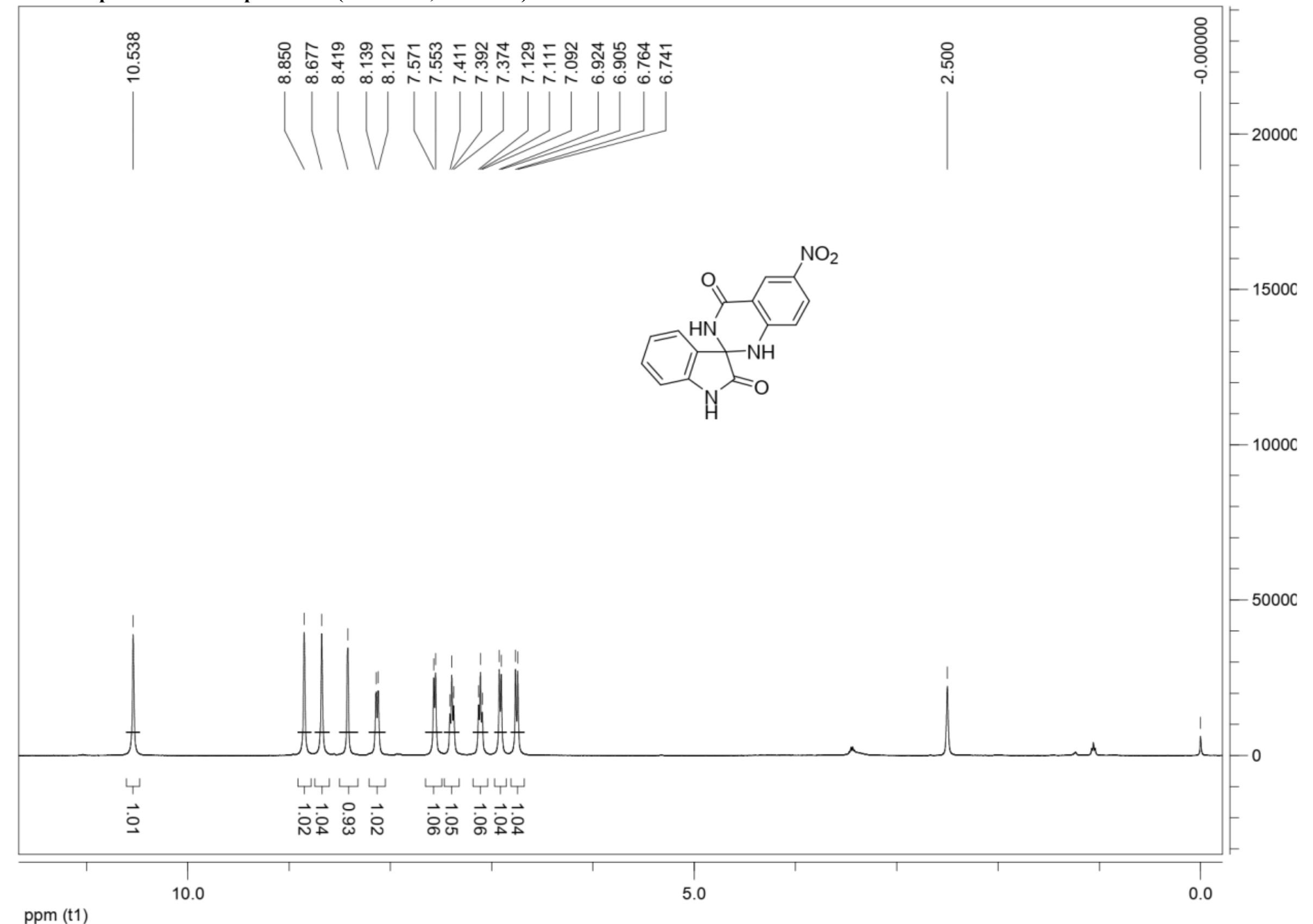


${ }^{13} \mathrm{C}$ NMR spectrum of Compound 3w (DMSO- $d_{6}, 100 \mathrm{MHz}$ )

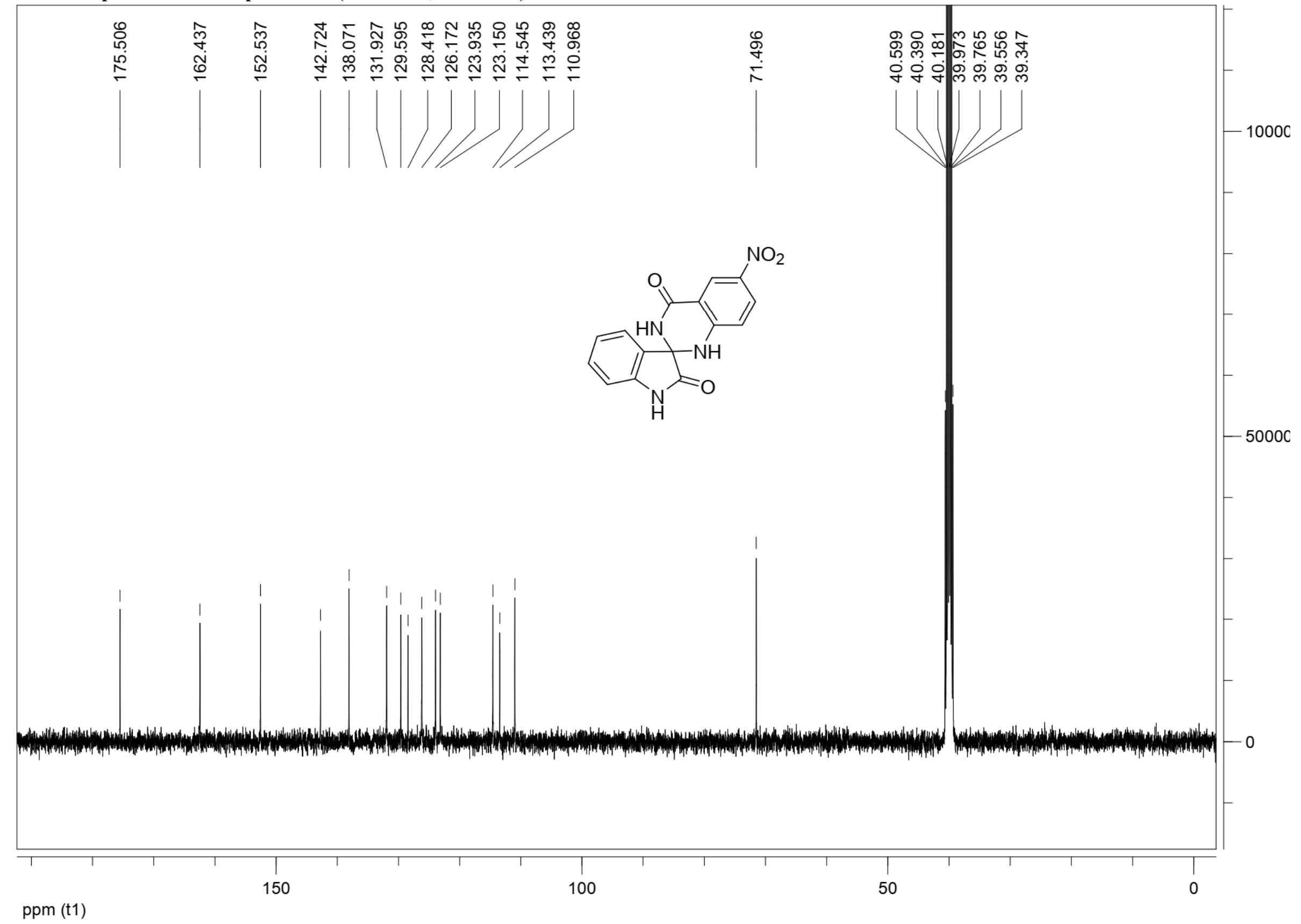




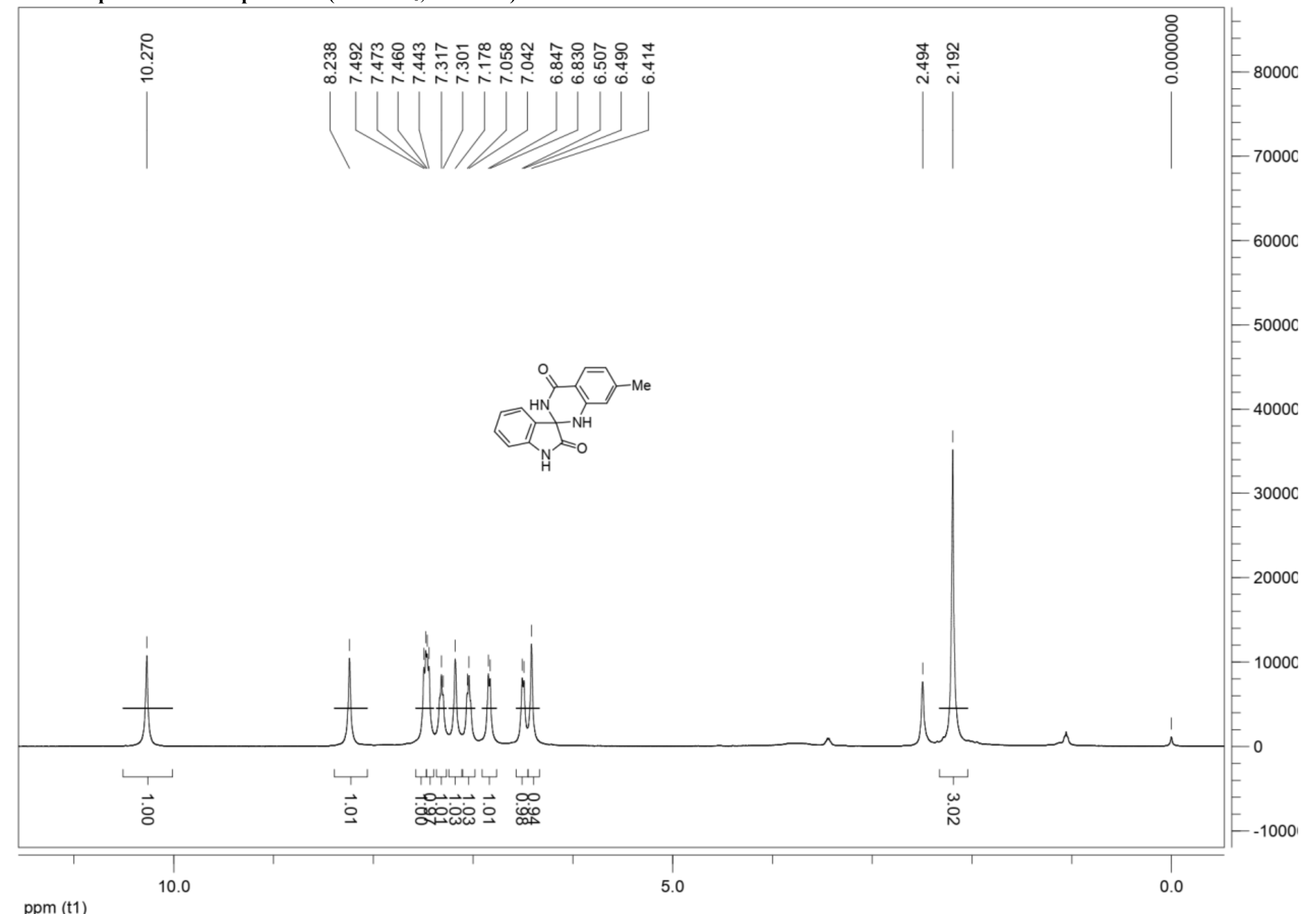




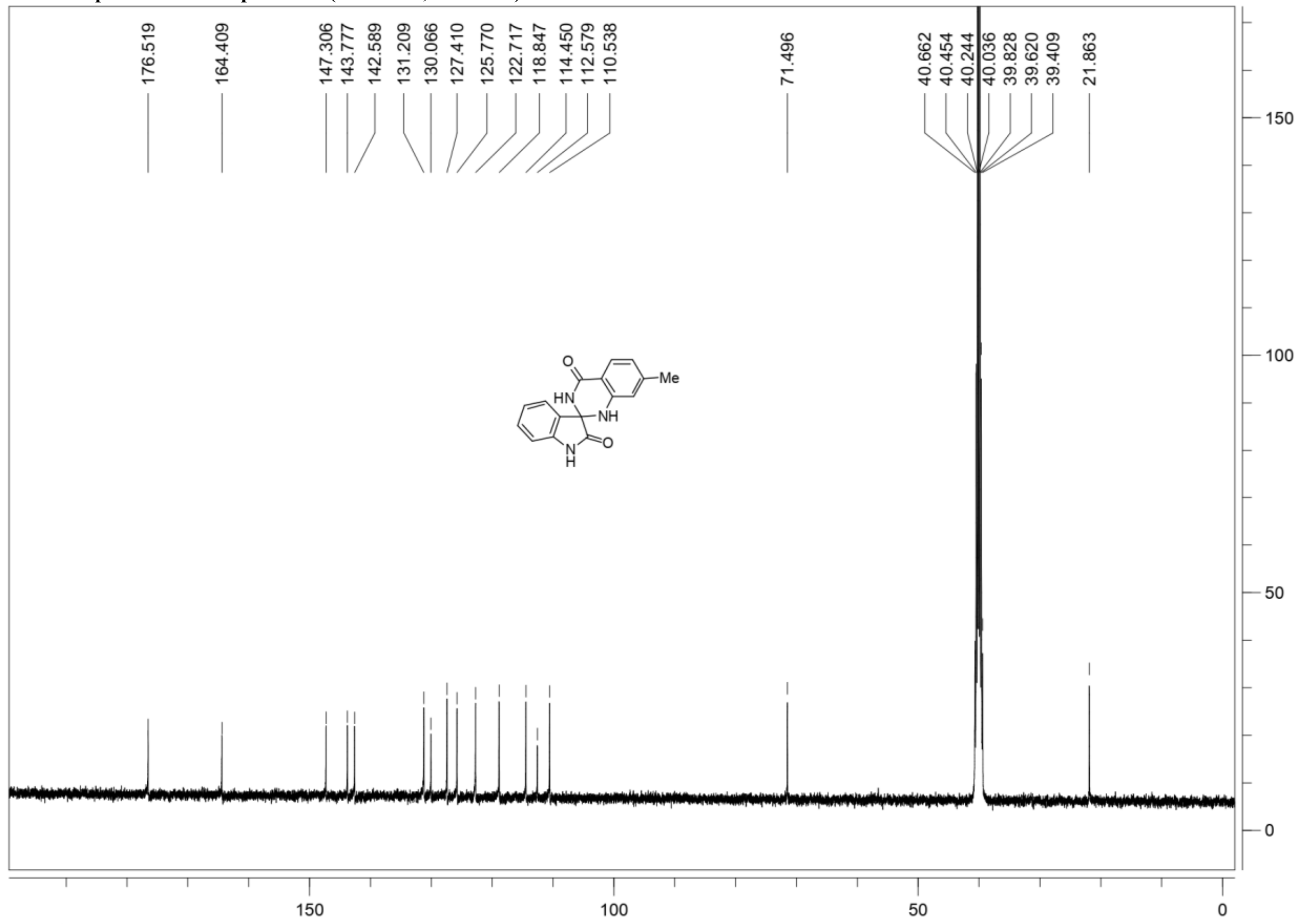

ppm (t1) 


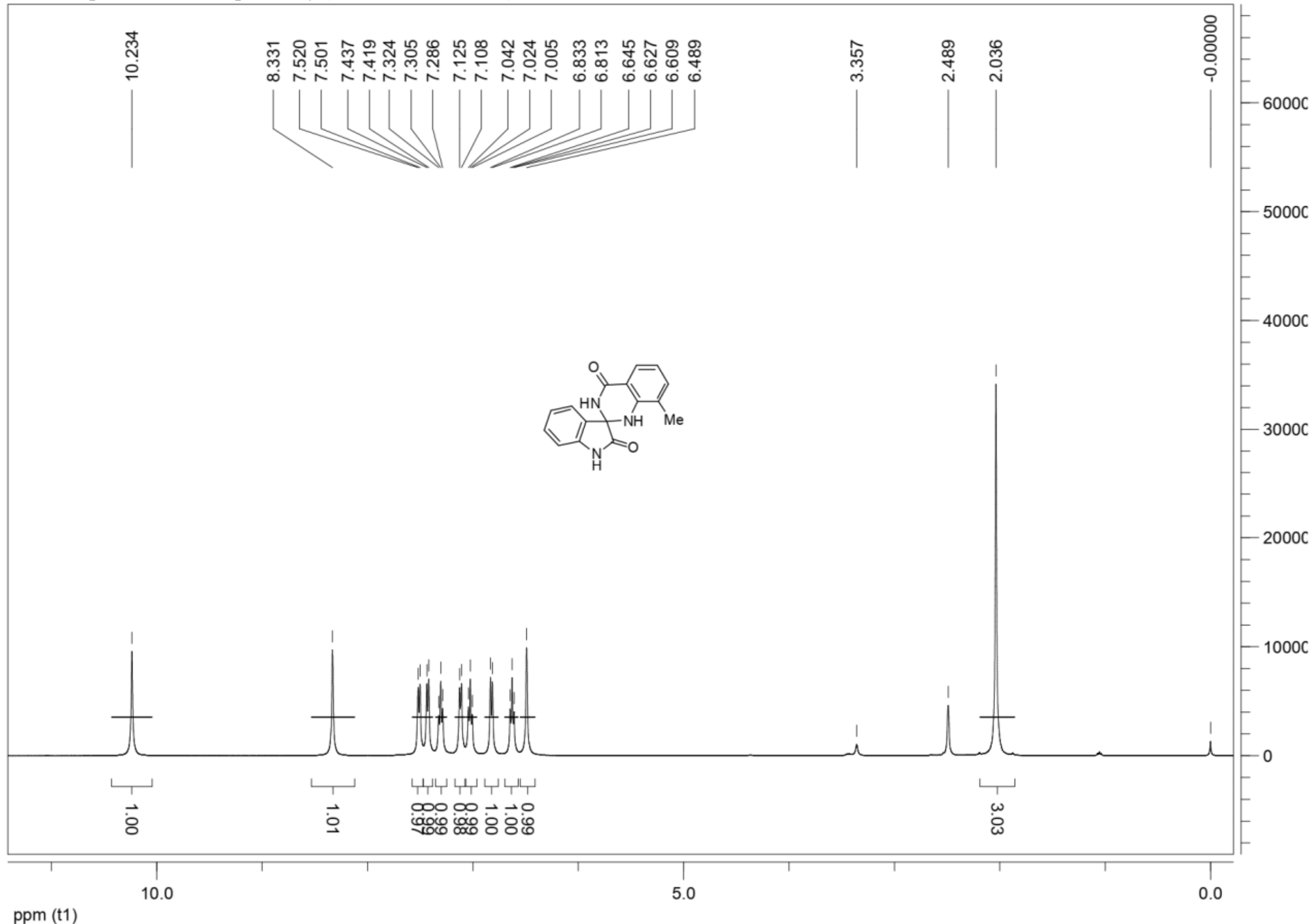


${ }^{13} \mathrm{C}$ NMR spectrum of Compound $3 \mathrm{y}$ (DMSO- $\left.d_{6}, 100 \mathrm{MHz}\right)$

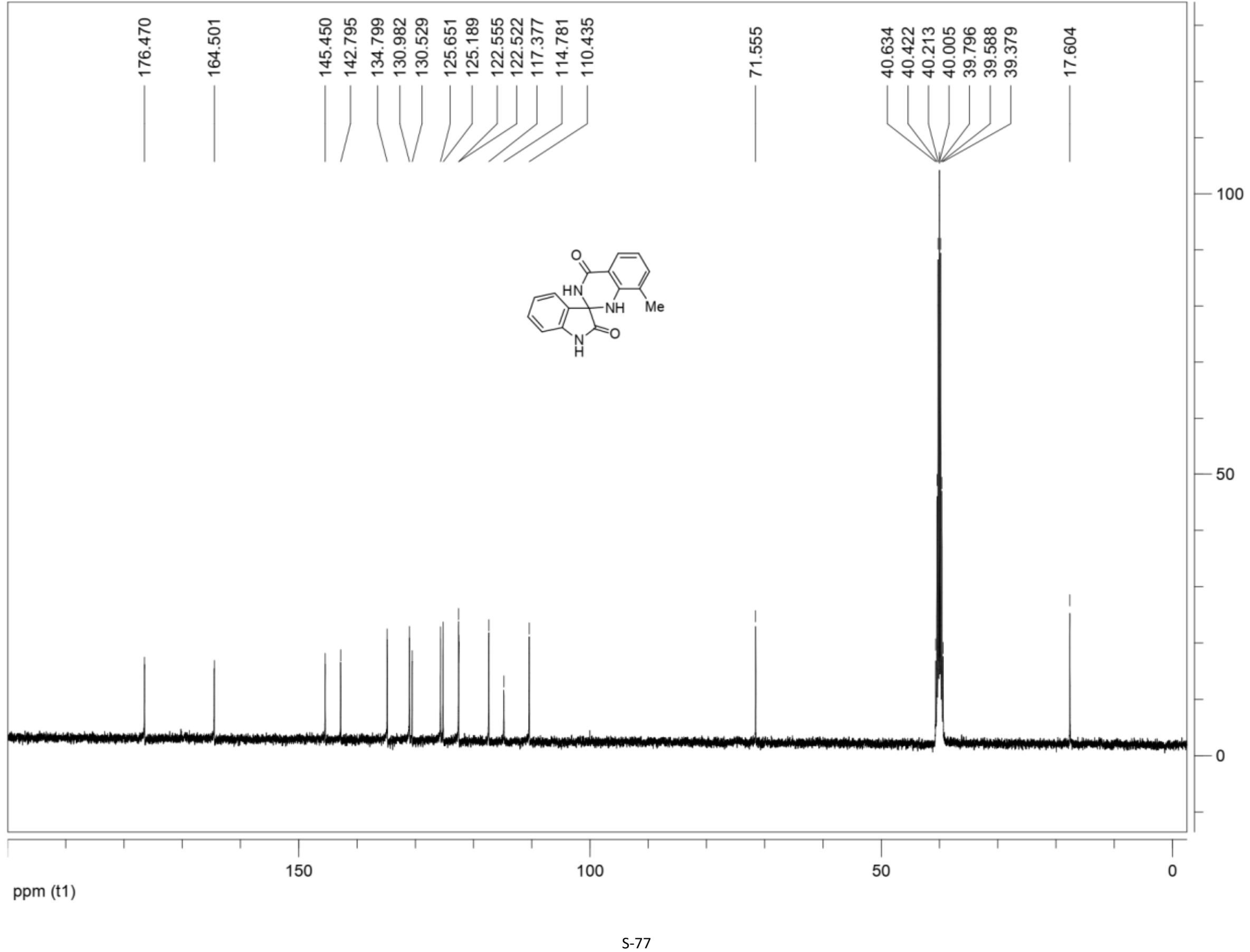




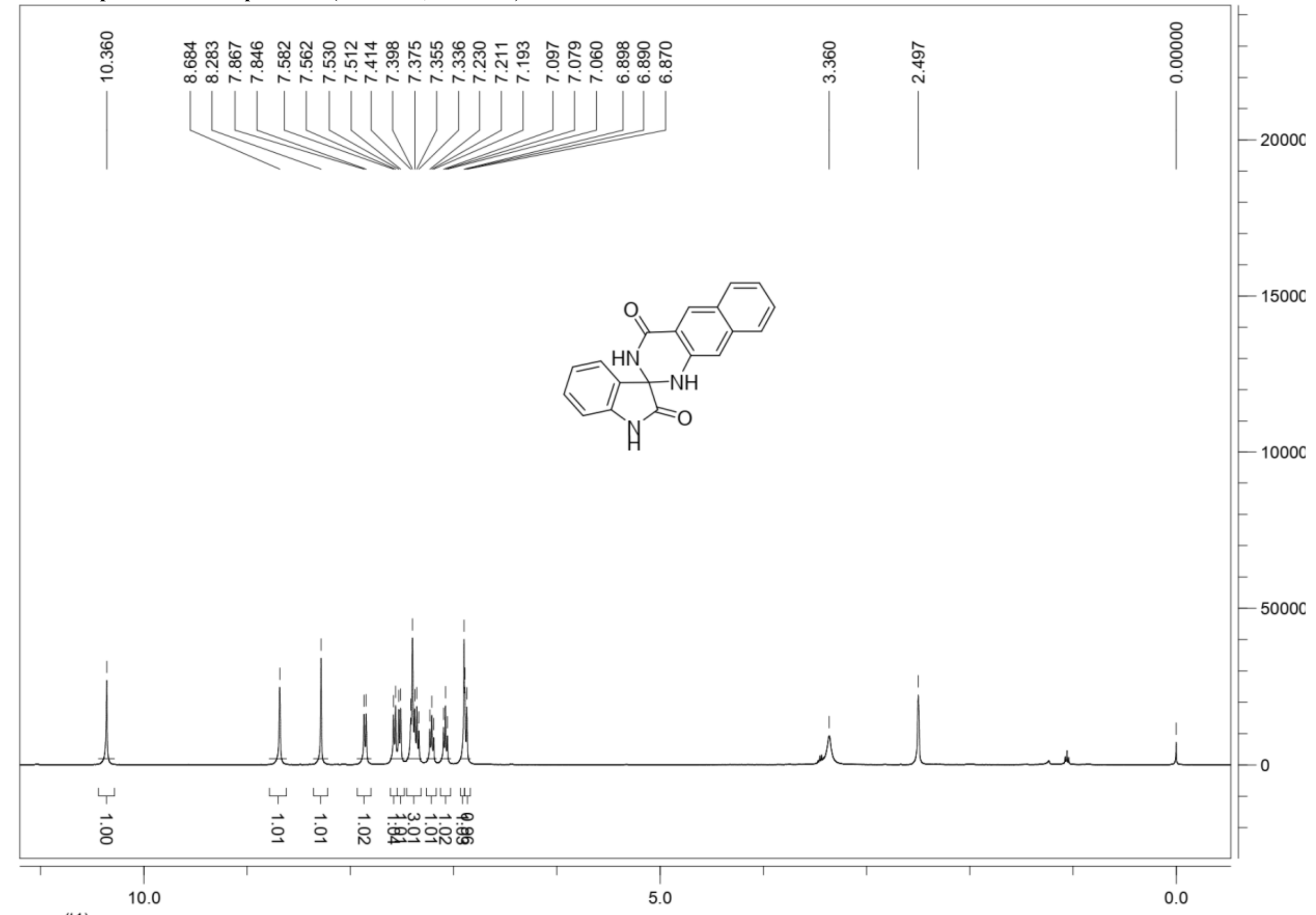

ppm (t1) 


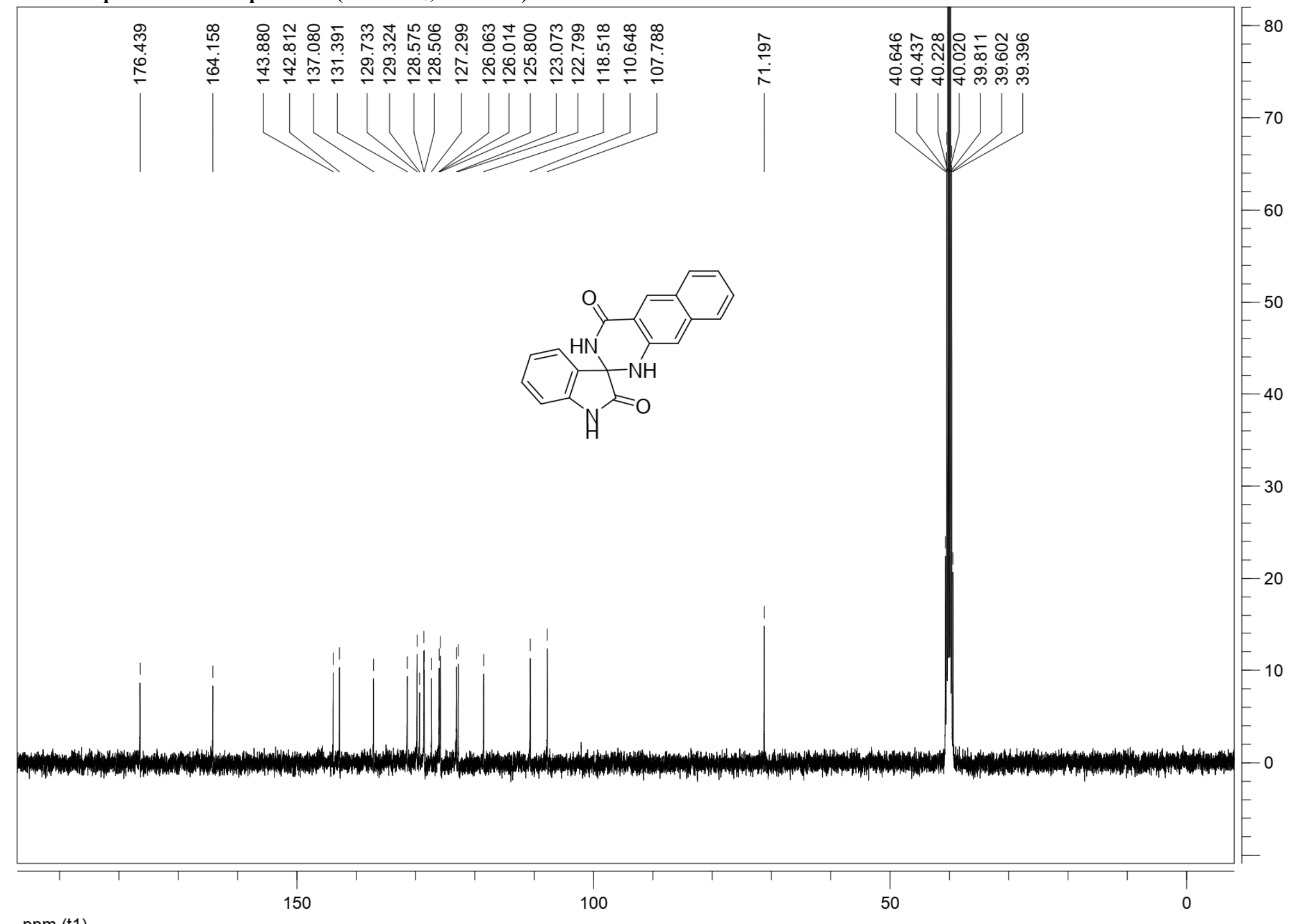

ppm (t1) 
${ }^{1} \mathrm{H}$ NMR spectrum of Compound 3aa (DMSO- $d_{6}, 400 \mathrm{MHz}$ )

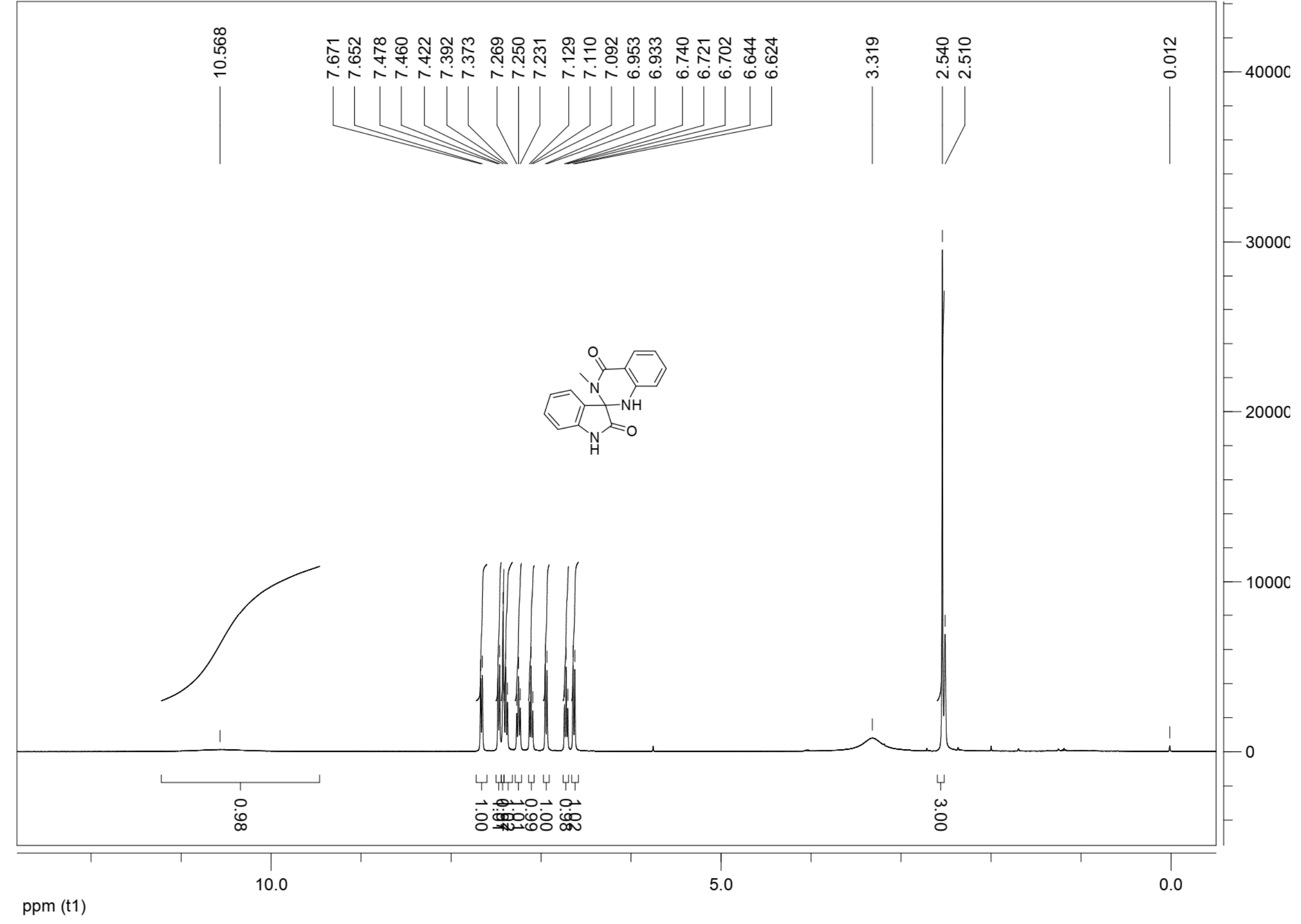




\section{${ }^{13} \mathrm{C}$ NMR spectrum of Compound 3aa (DMSO- $d_{6}, 100 \mathrm{MHz}$ )}

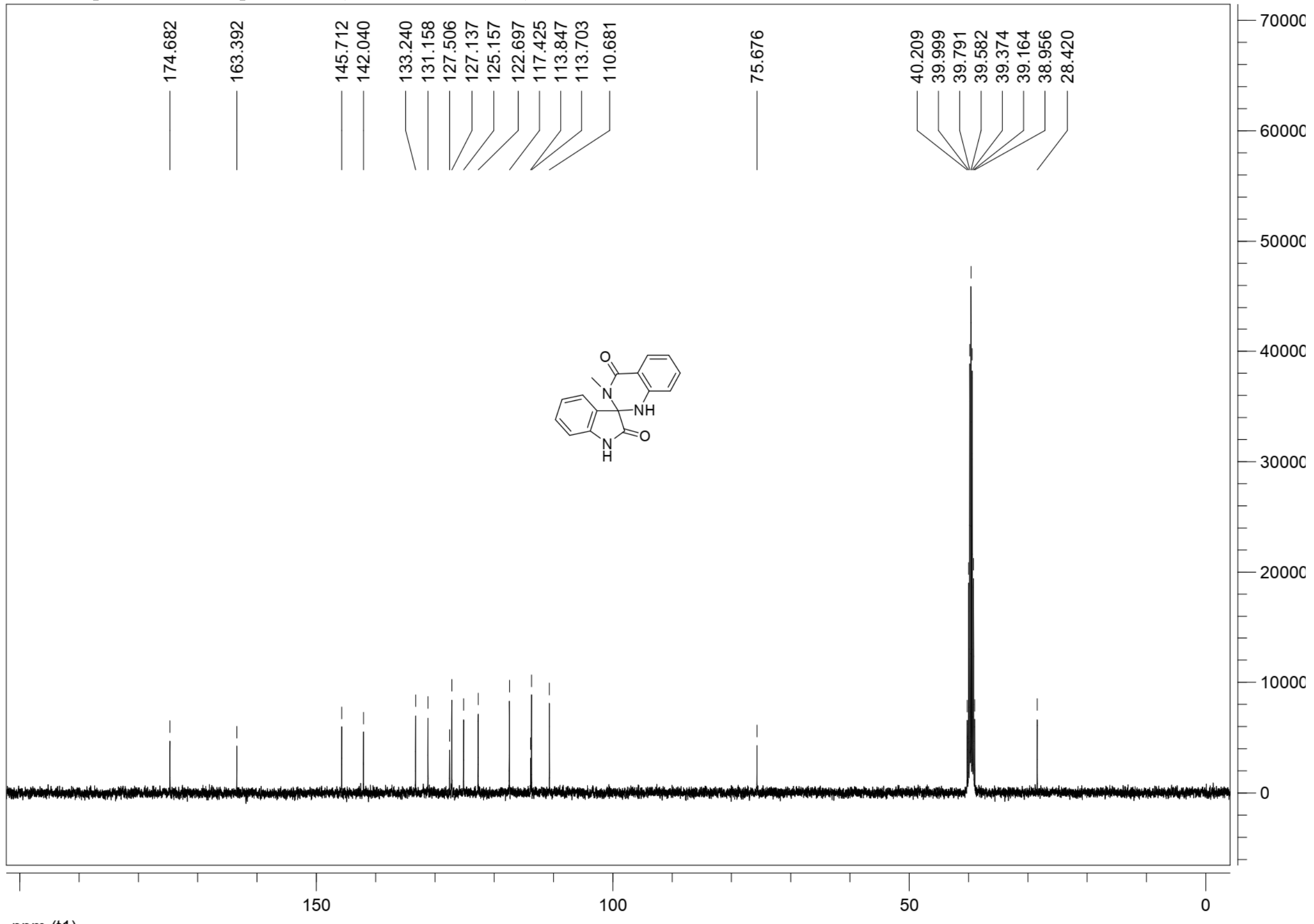

ppm (t1) 
${ }^{1} \mathrm{H}$ NMR spectrum of Compound 3ab (DMSO- $d_{6}, 400 \mathrm{MHz}$ )

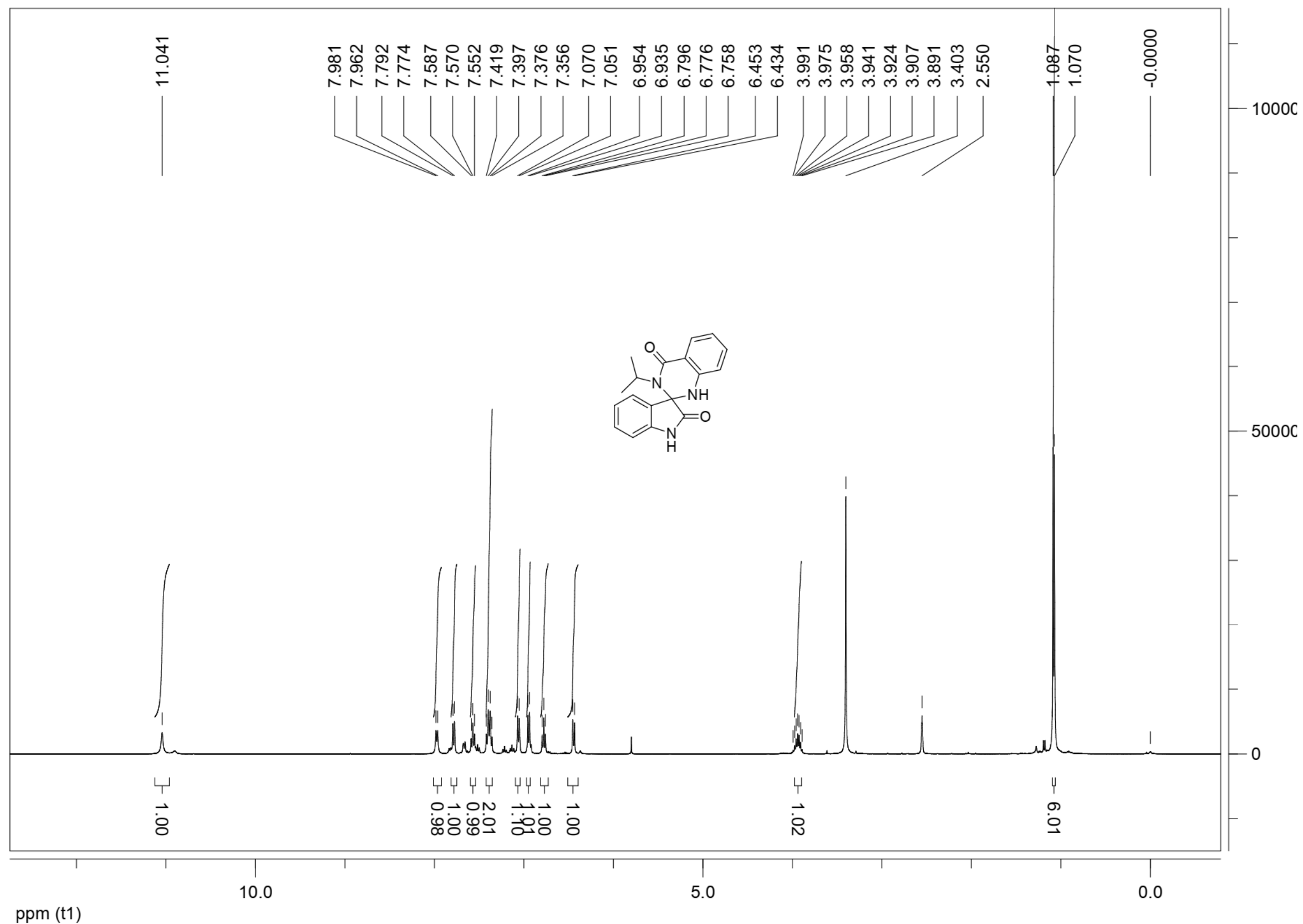


${ }^{13} \mathrm{C}$ NMR spectrum of Compound 3ab (DMSO- $d_{6}, 100 \mathrm{MHz}$ )

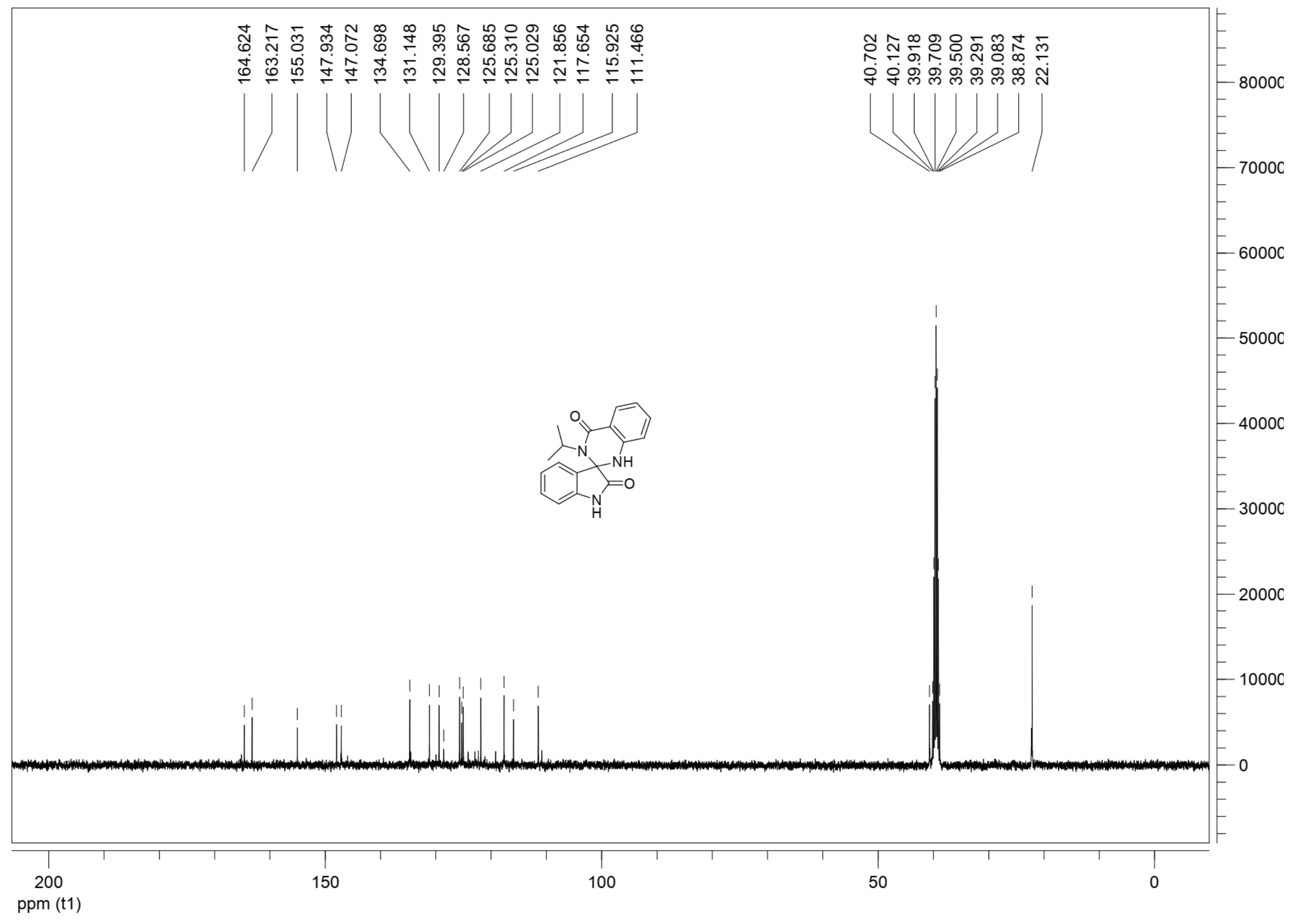


${ }^{1} \mathrm{H}$ NMR spectrum of Compound 3ac (DMSO- $d_{6}, 400 \mathrm{MHz}$ )

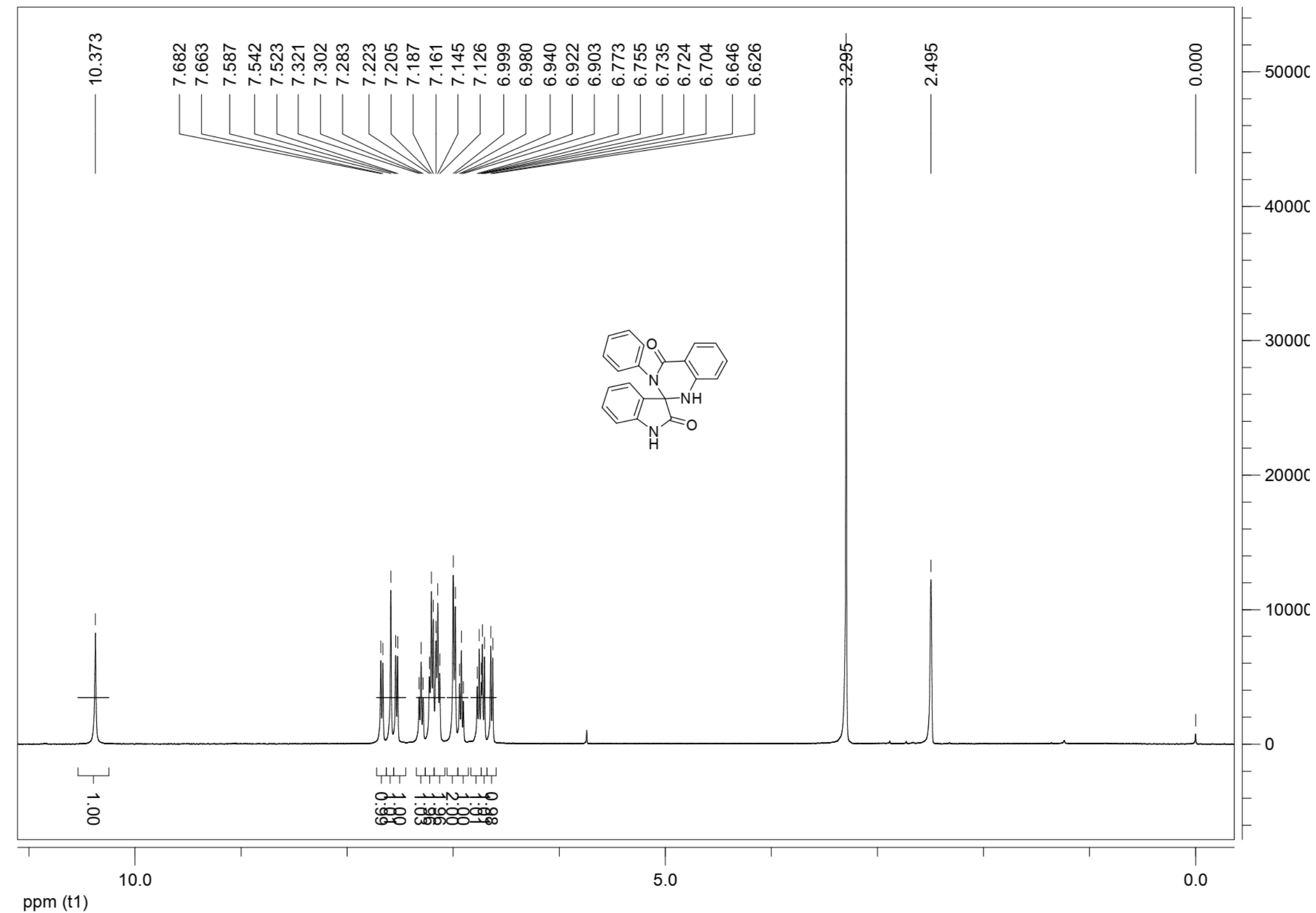


${ }^{13} \mathrm{C}$ NMR spectrum of Compound 3ac (DMSO- $d_{6}, 100 \mathrm{MHz}$ )

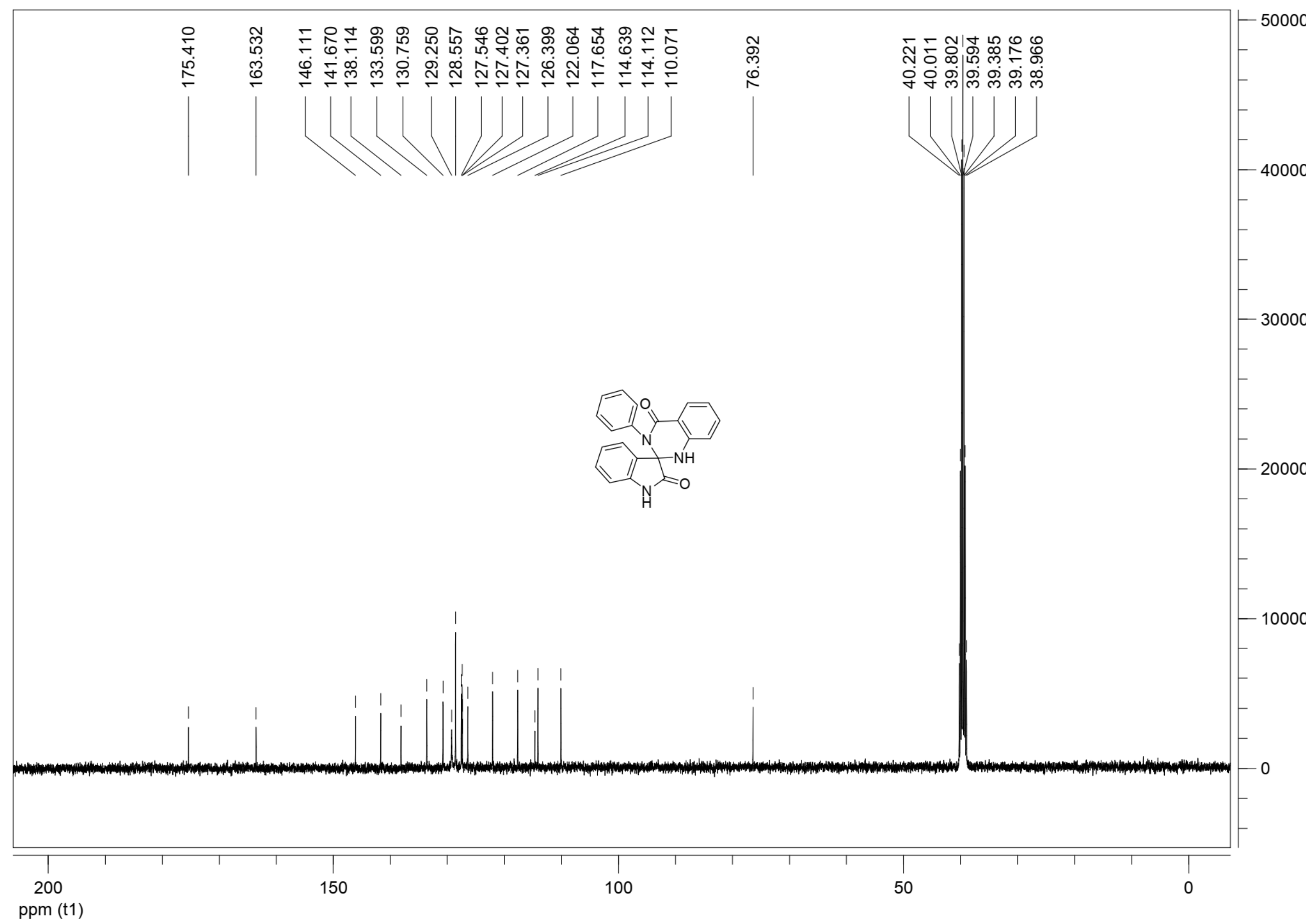




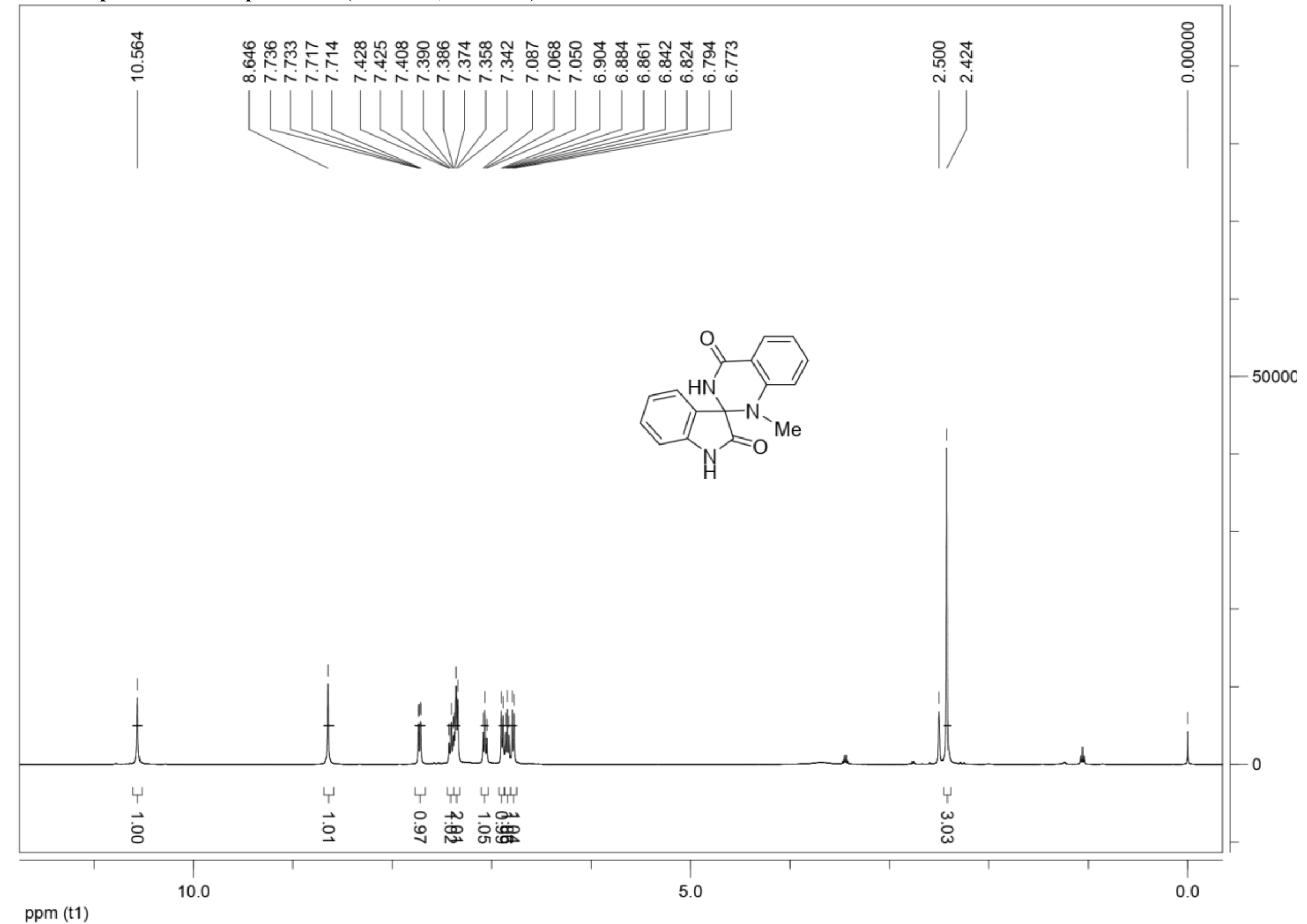


${ }^{13} \mathrm{C}$ NMR spectrum of Compound 3ad (DMSO- $d_{6}, 100 \mathrm{MHz}$ )

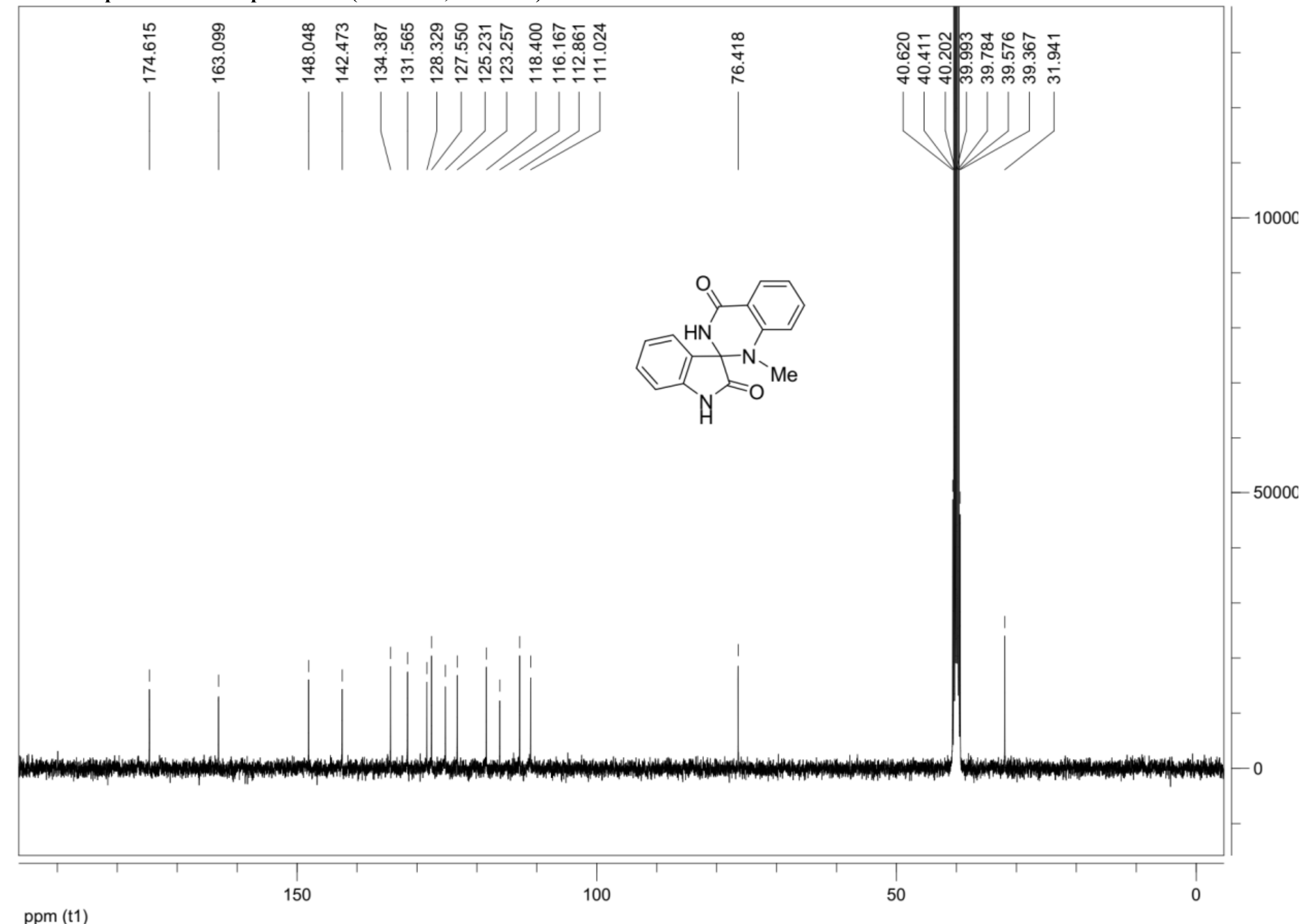




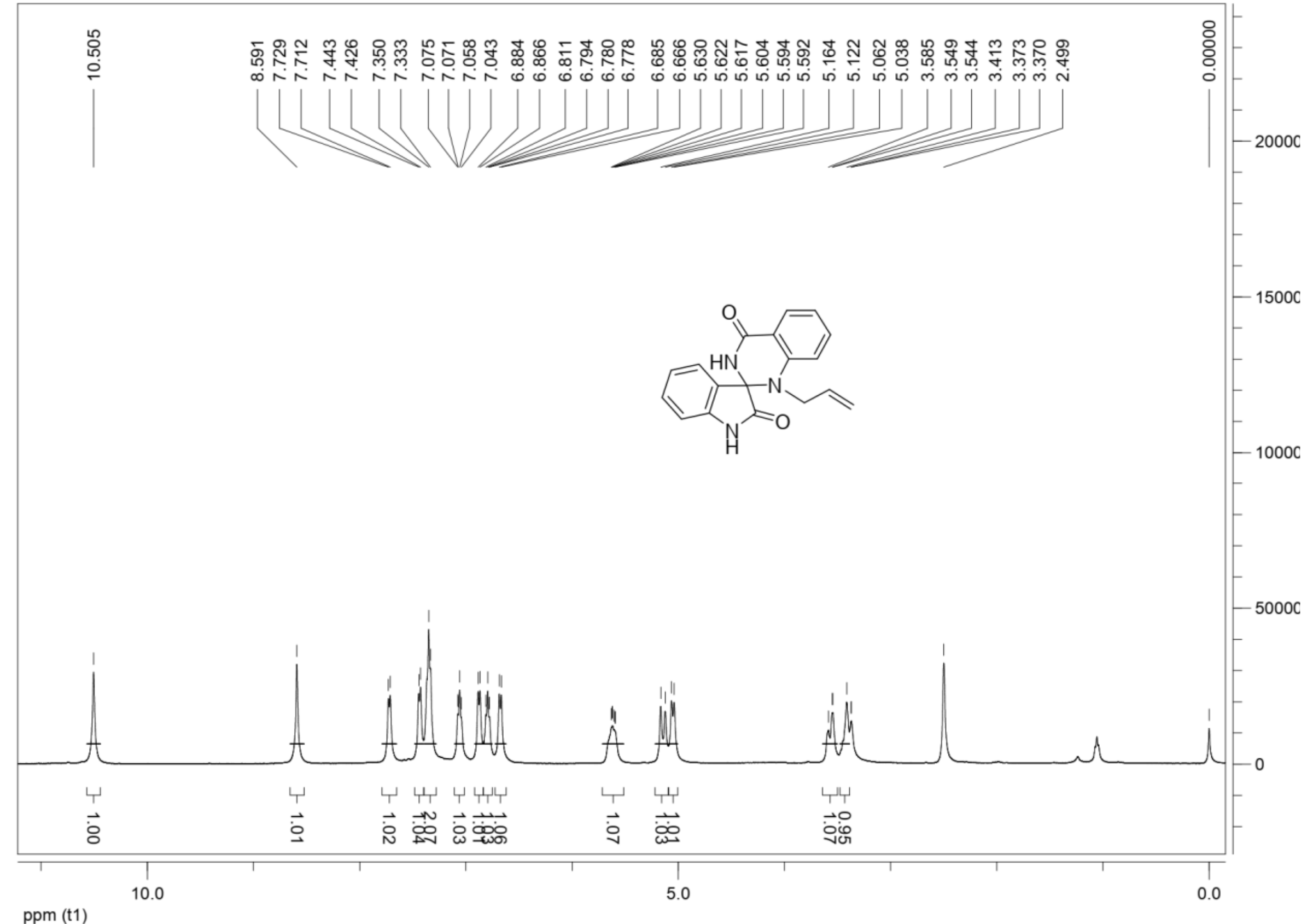




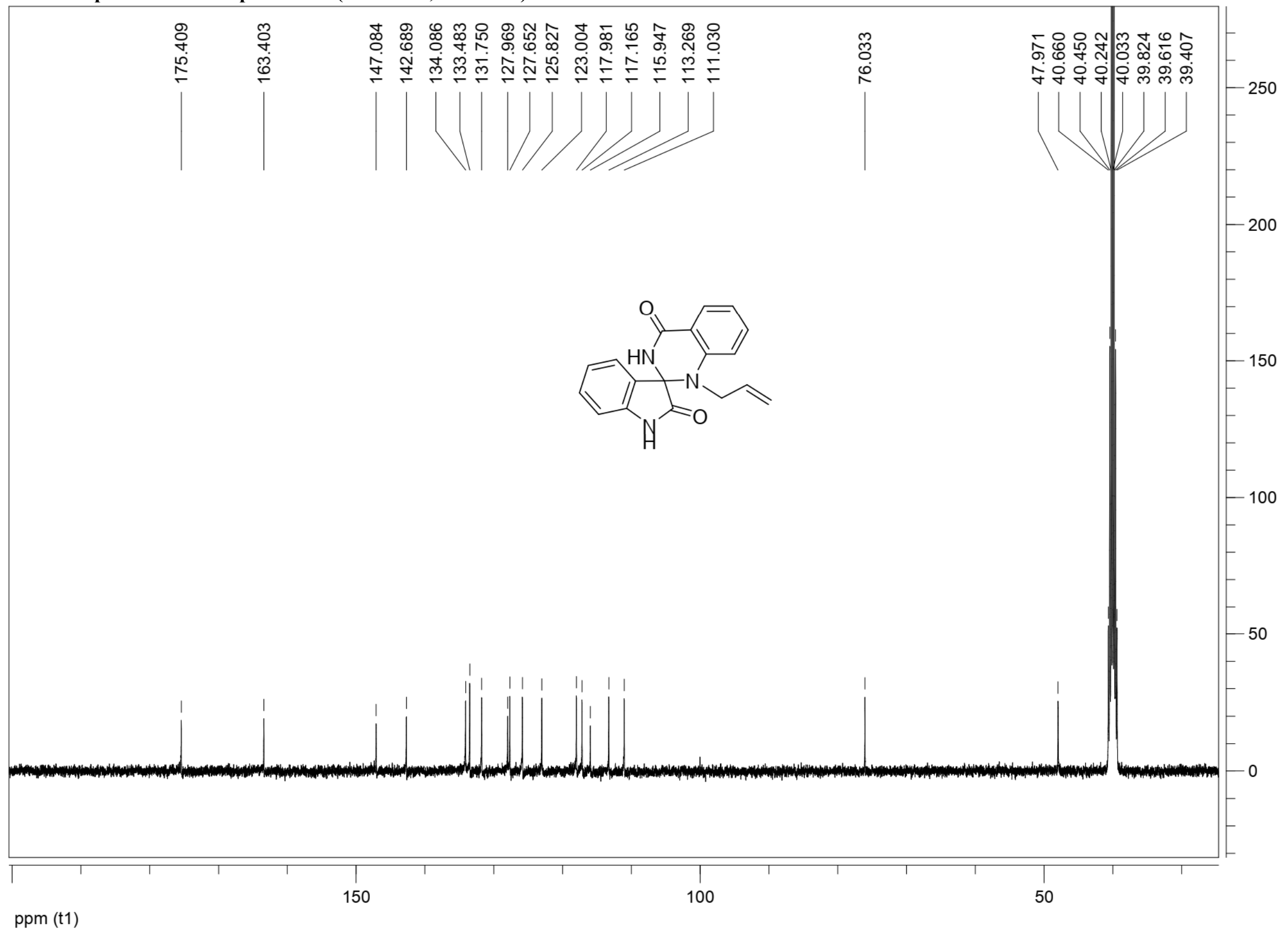




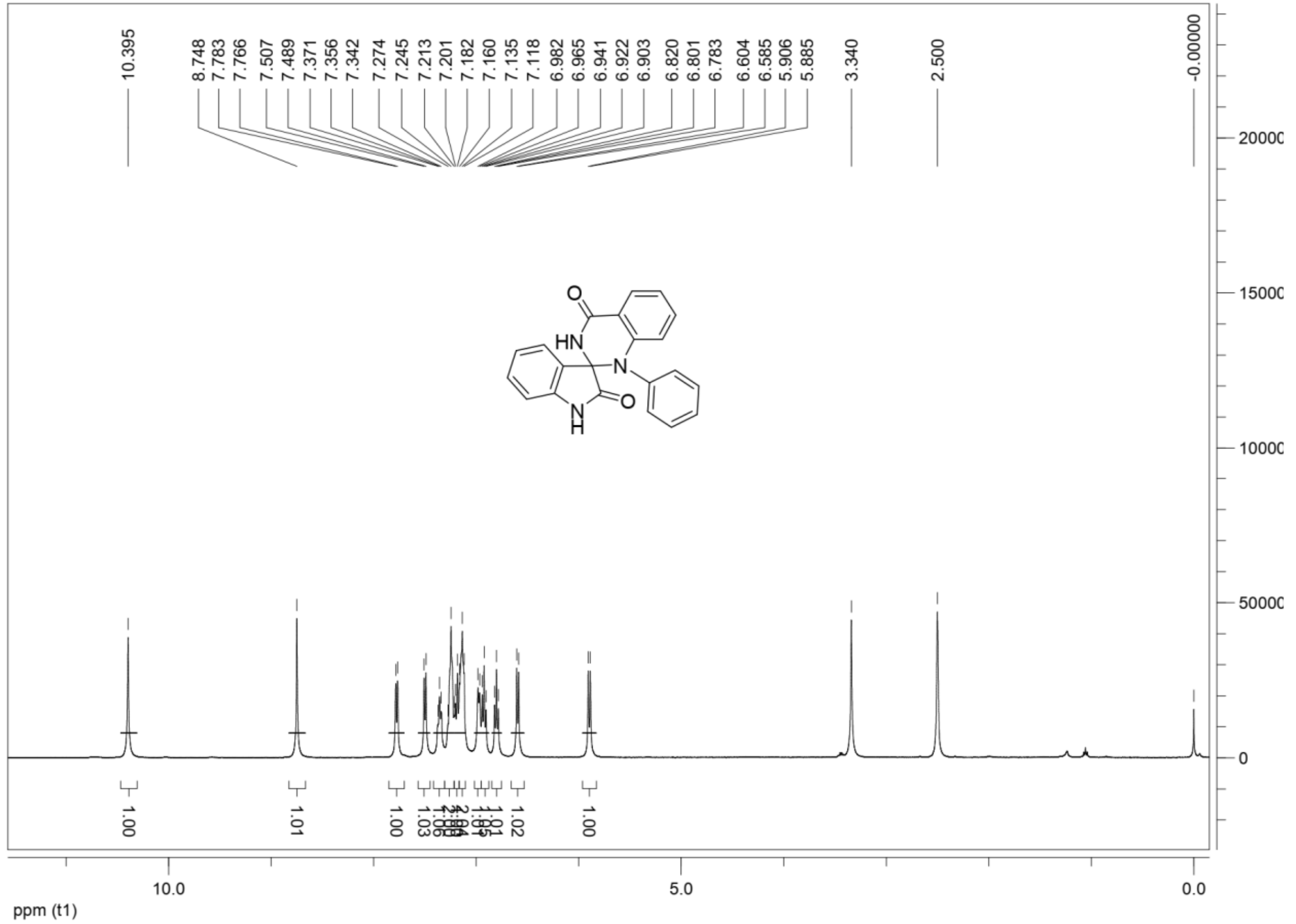


${ }^{13} \mathrm{C}$ NMR spectrum of Compound 3af (DMSO- $d_{6}, 100 \mathrm{MHz}$ )

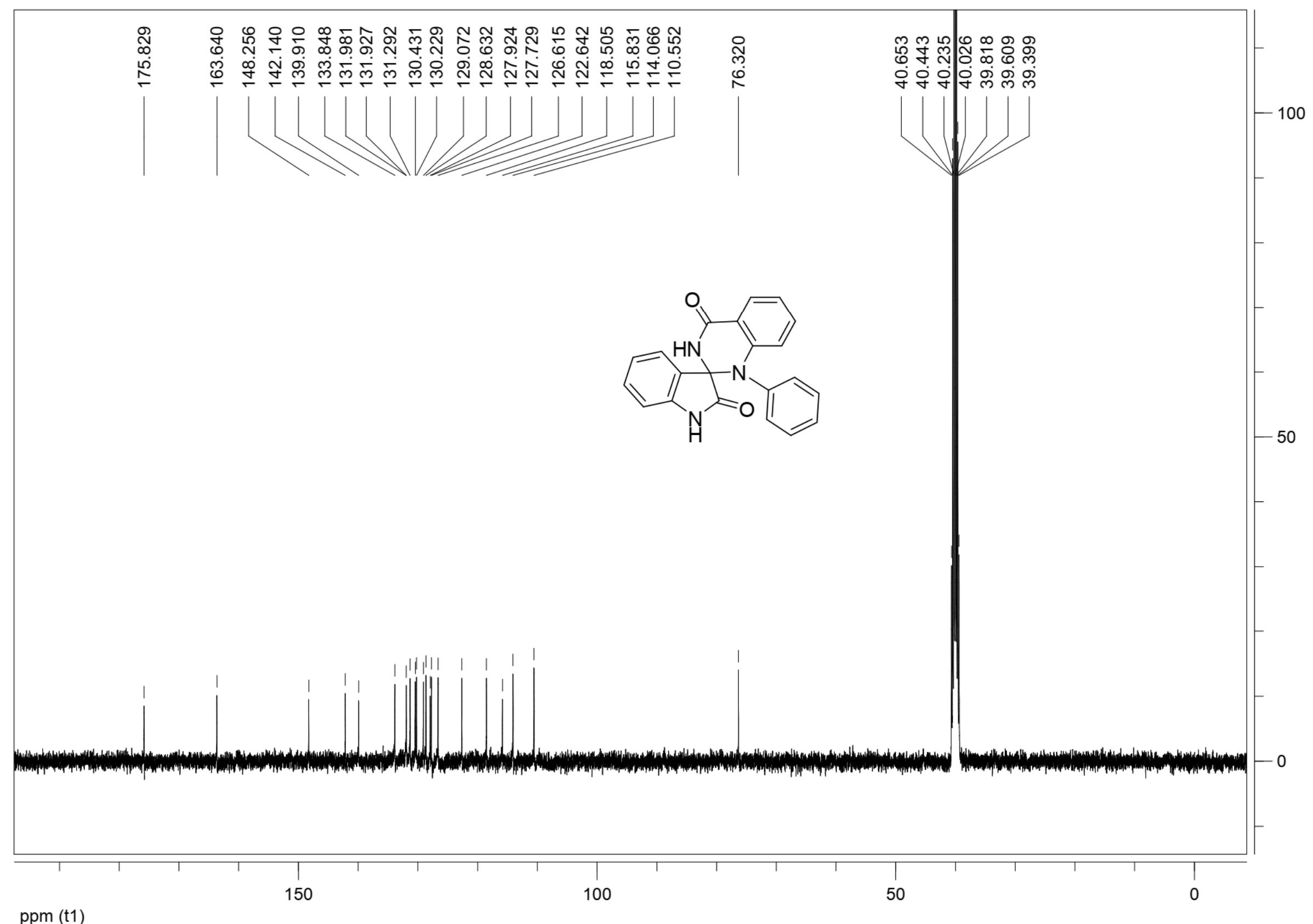

ppm (t1) 
${ }^{1} \mathrm{H}$ NMR spectrum of Compound 3ah (DMSO- $d_{6}, 400 \mathrm{MHz}$ )

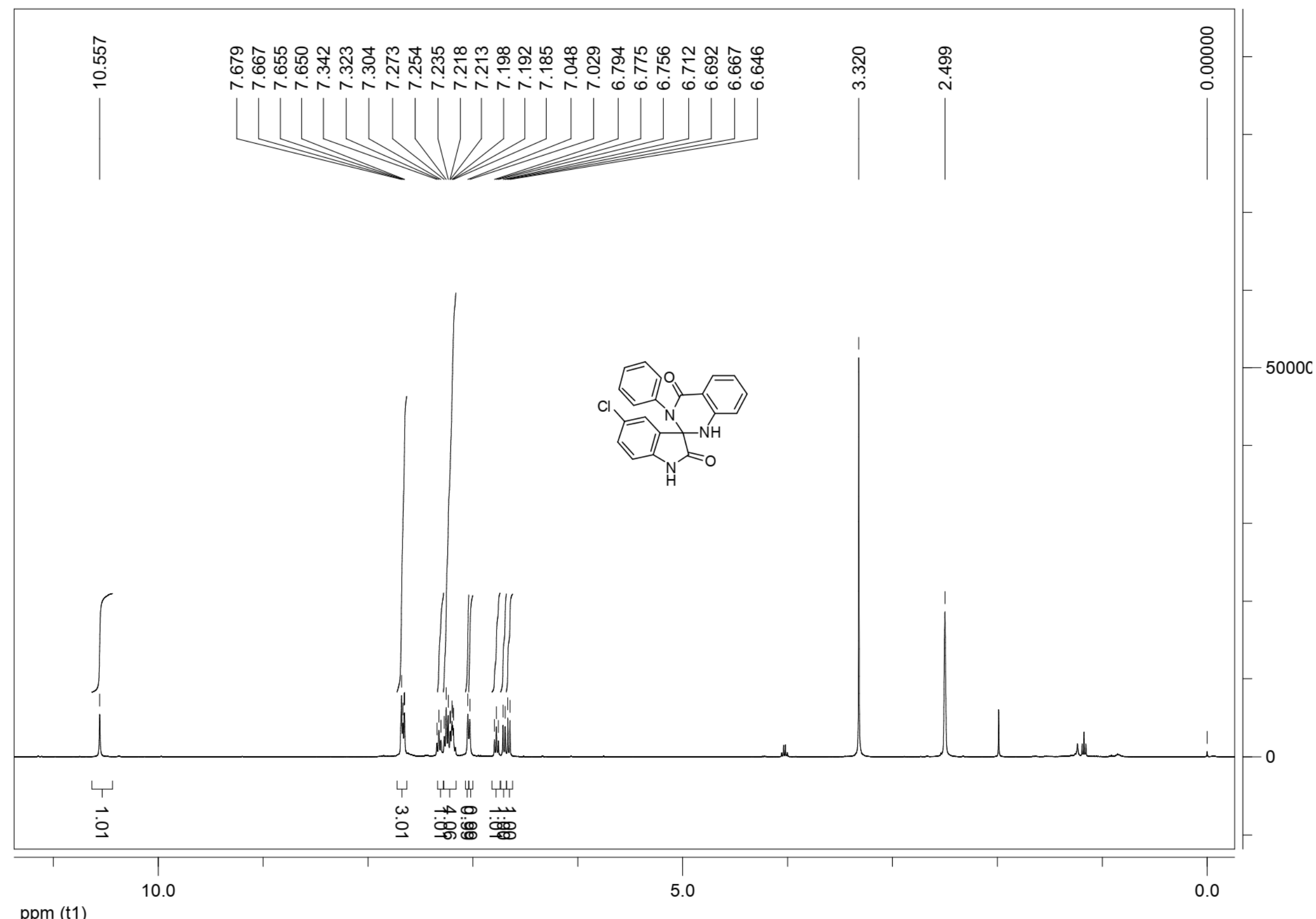


${ }^{13} \mathrm{C}$ NMR spectrum of Compound 3ah (DMSO- $d_{6}, 100 \mathrm{MHz}$ )

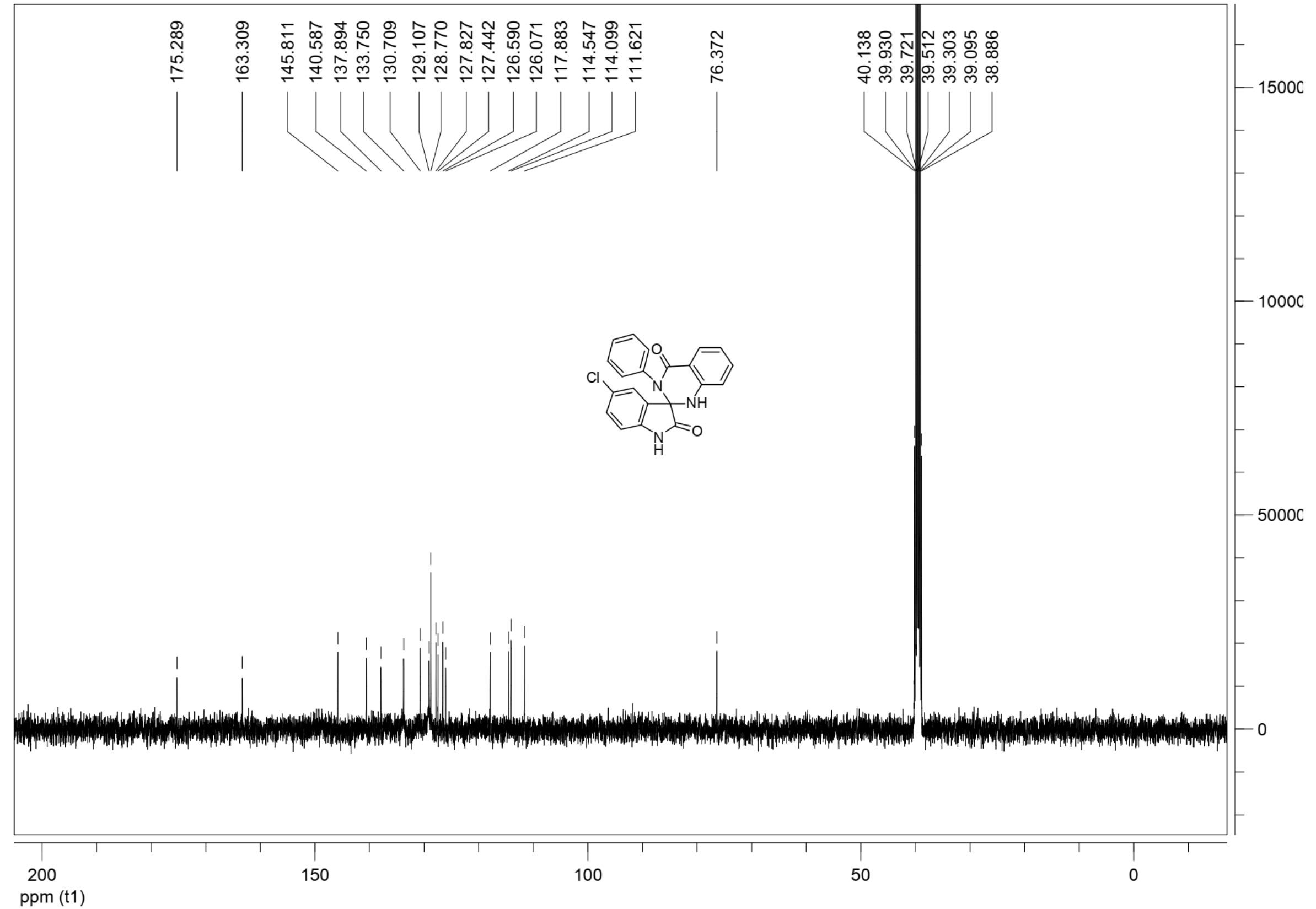




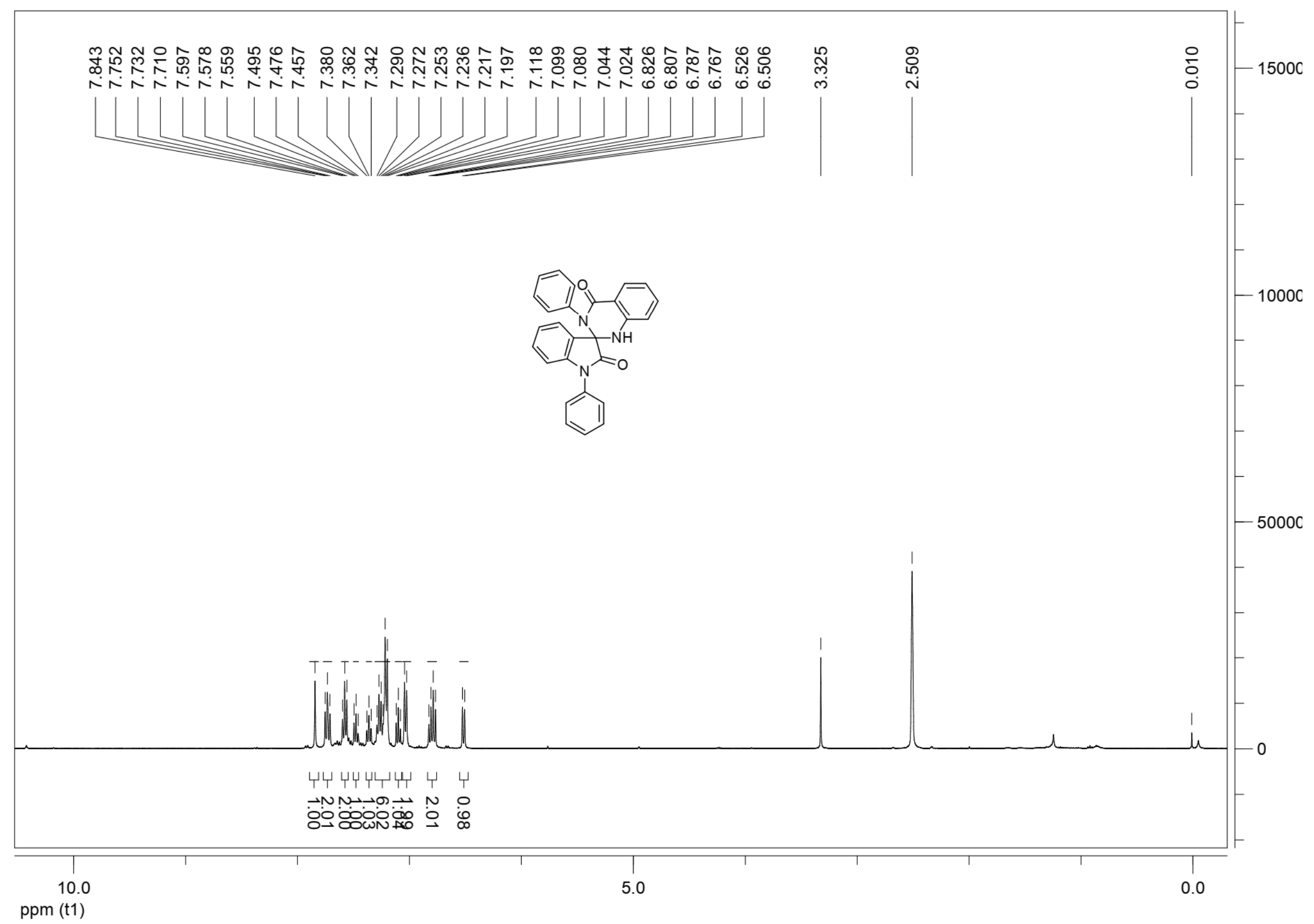


${ }^{13} \mathrm{C}$ NMR spectrum of Compound 3ai (DMSO- $d_{6}, 100 \mathrm{MHz}$ )

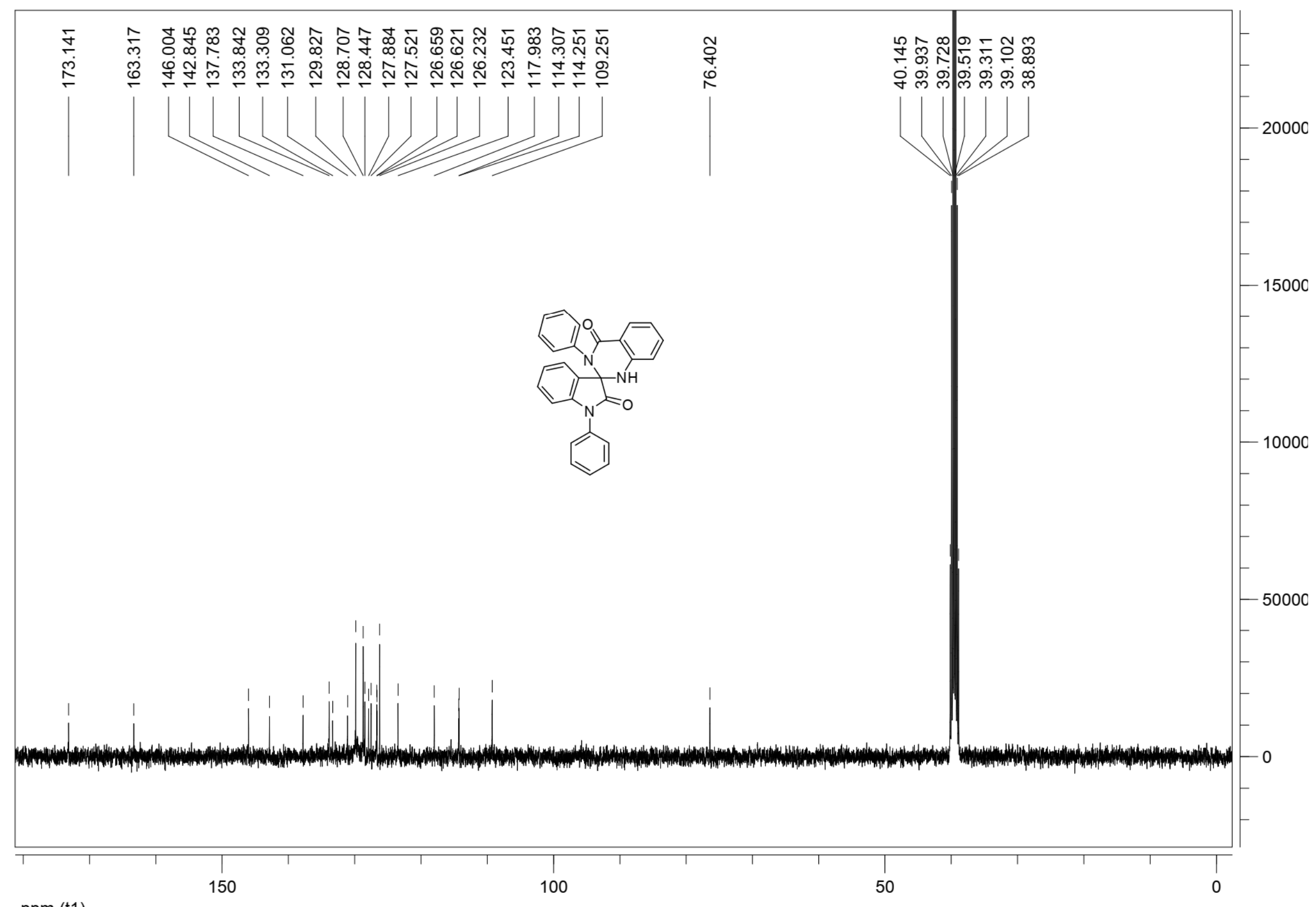

ppm (t1) 
${ }^{1} \mathrm{H}$ NMR spectrum of Compound 3aj (DMSO- $d_{6}, 400 \mathrm{MHz}$ )

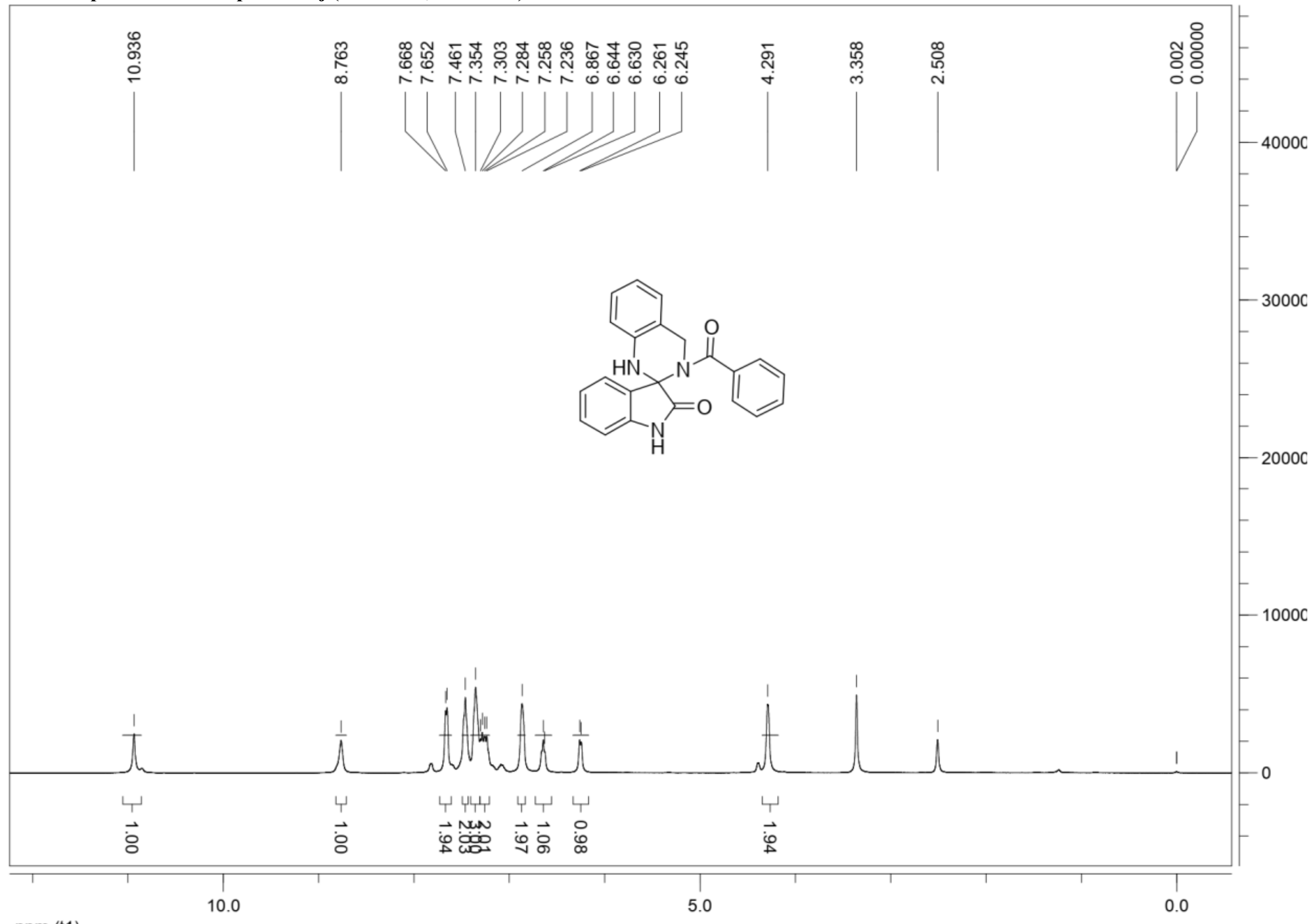

ppm (t1) 
${ }^{13} \mathrm{C}$ NMR spectrum of Compound 3aj (DMSO- $d_{6}, 100 \mathrm{MHz}$ )

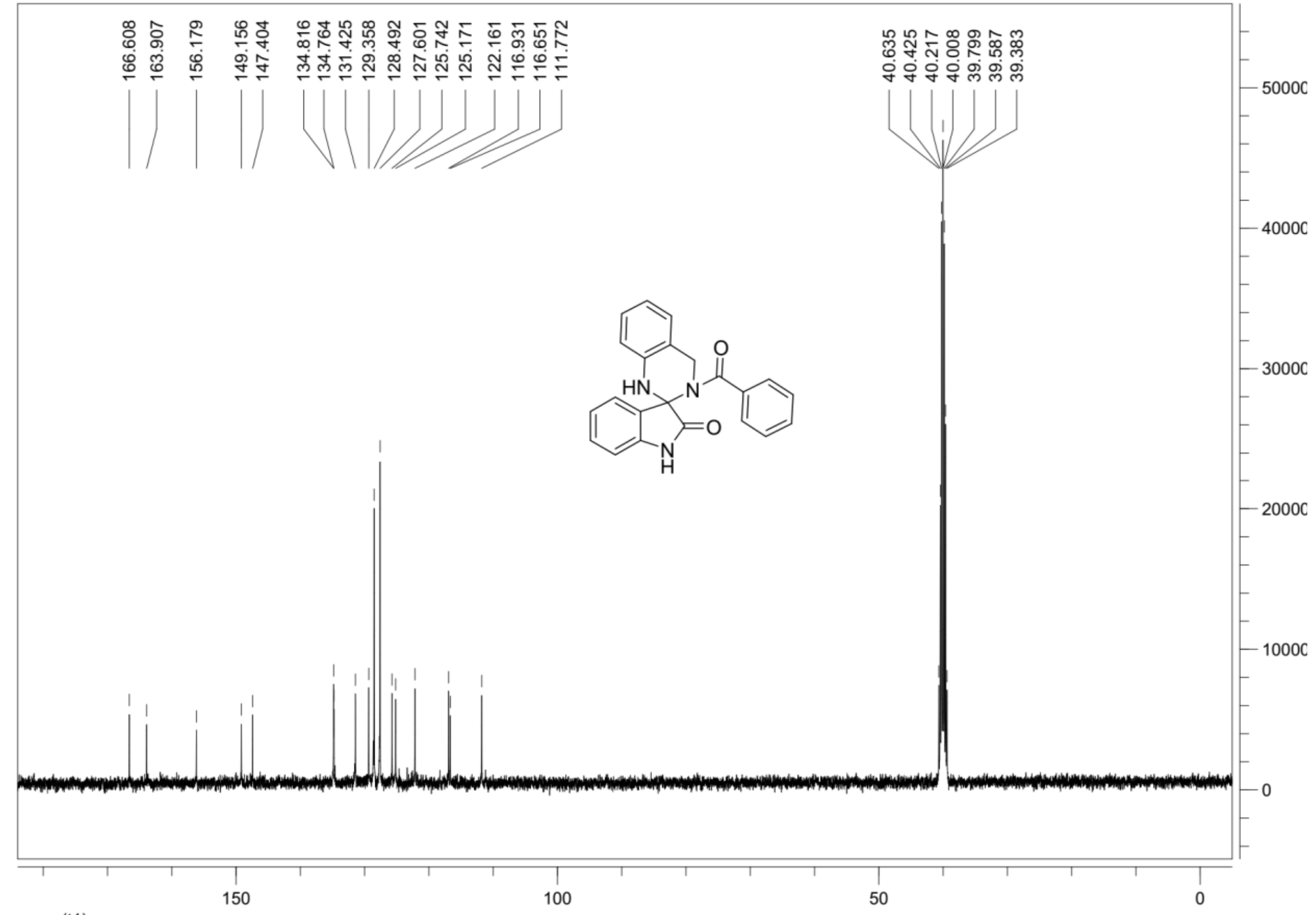

ppm (t1) 
${ }^{1} \mathrm{H}$ NMR spectrum of Compound 3ak (DMSO- $d_{6}, 400 \mathrm{MHz}$ )

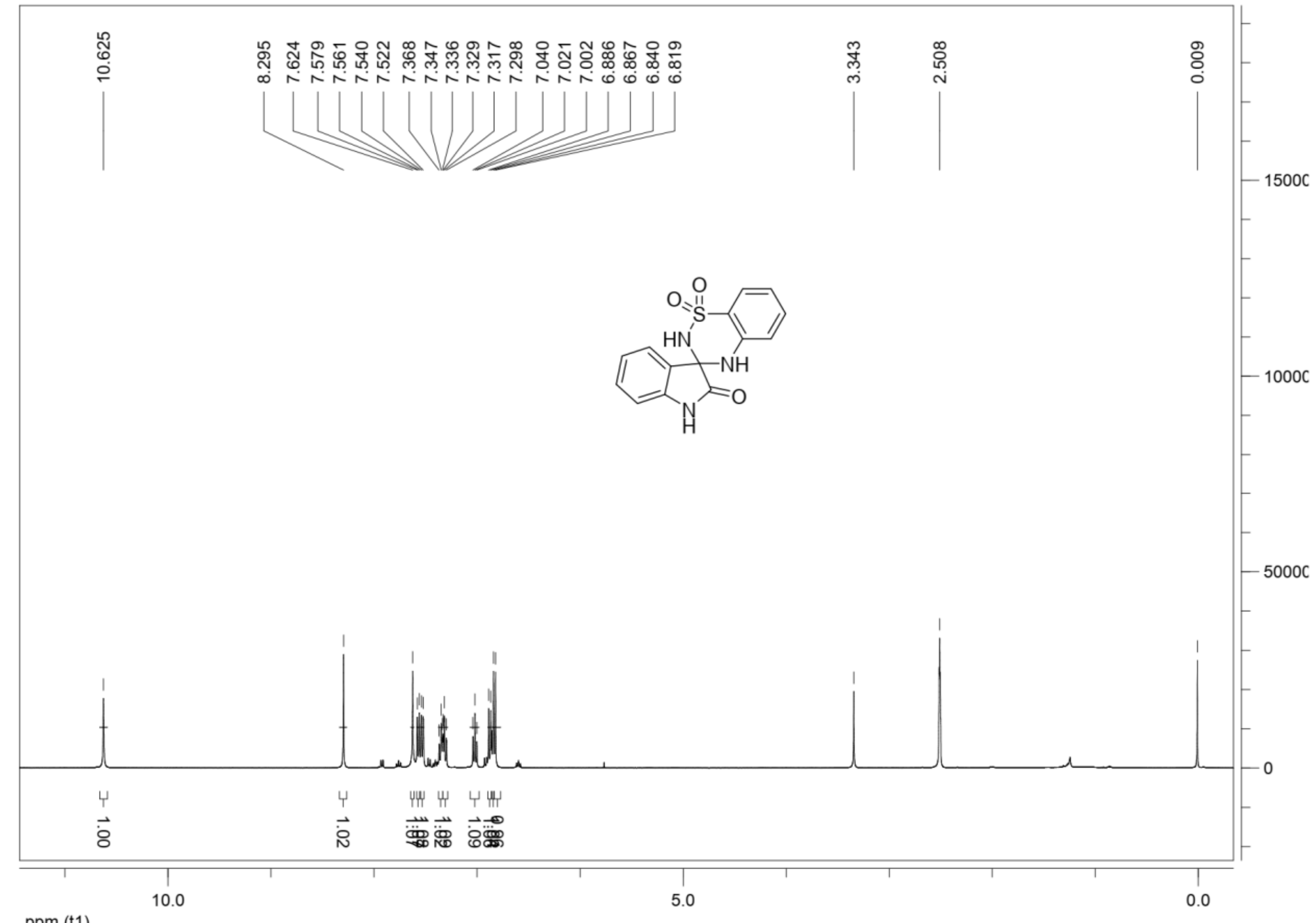

ppm (t1) 
${ }^{13} \mathrm{C}$ NMR spectrum of Compound 3ak (DMSO- $d_{6}, 100 \mathrm{MHz}$ )

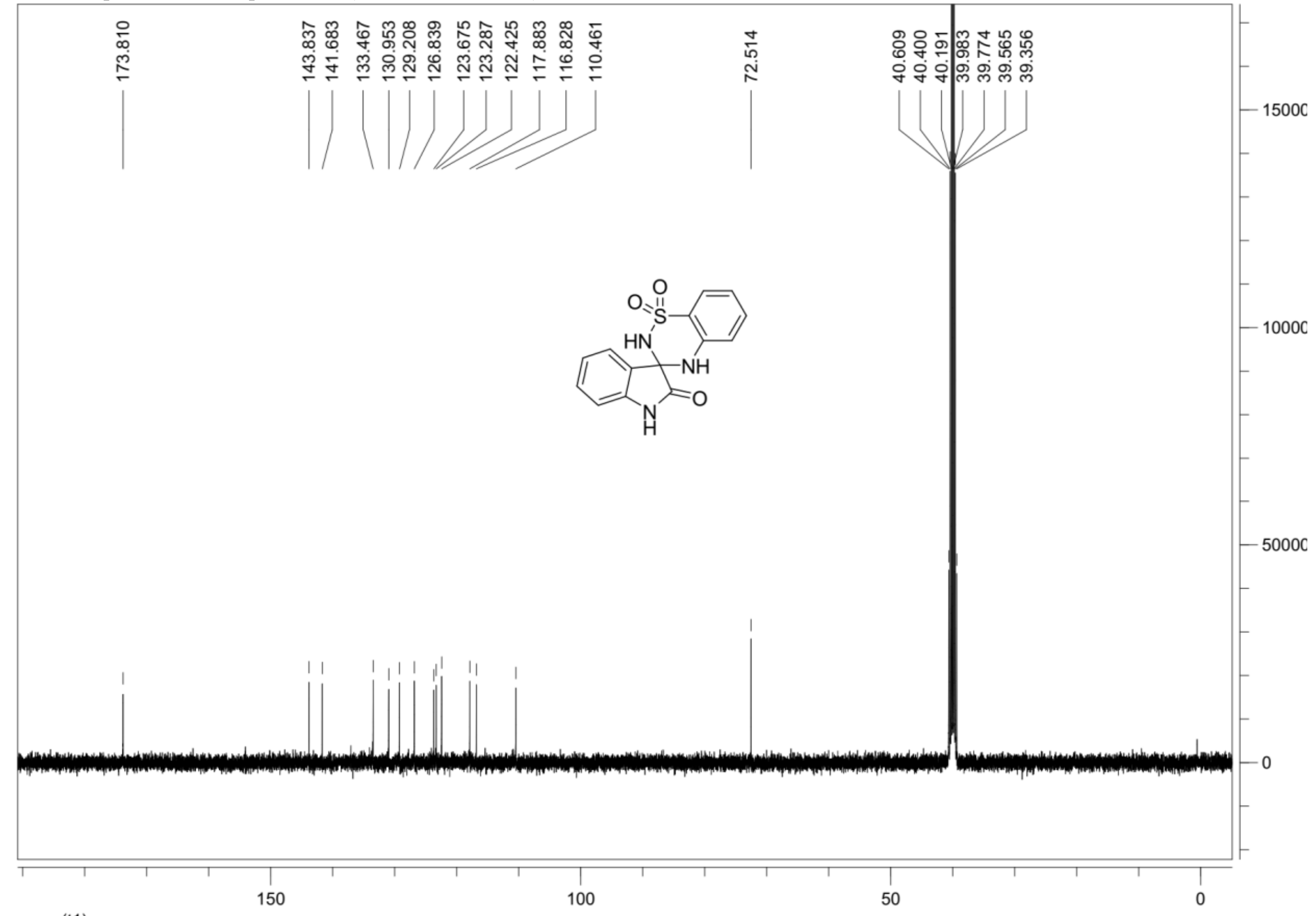

ppm (t1) 


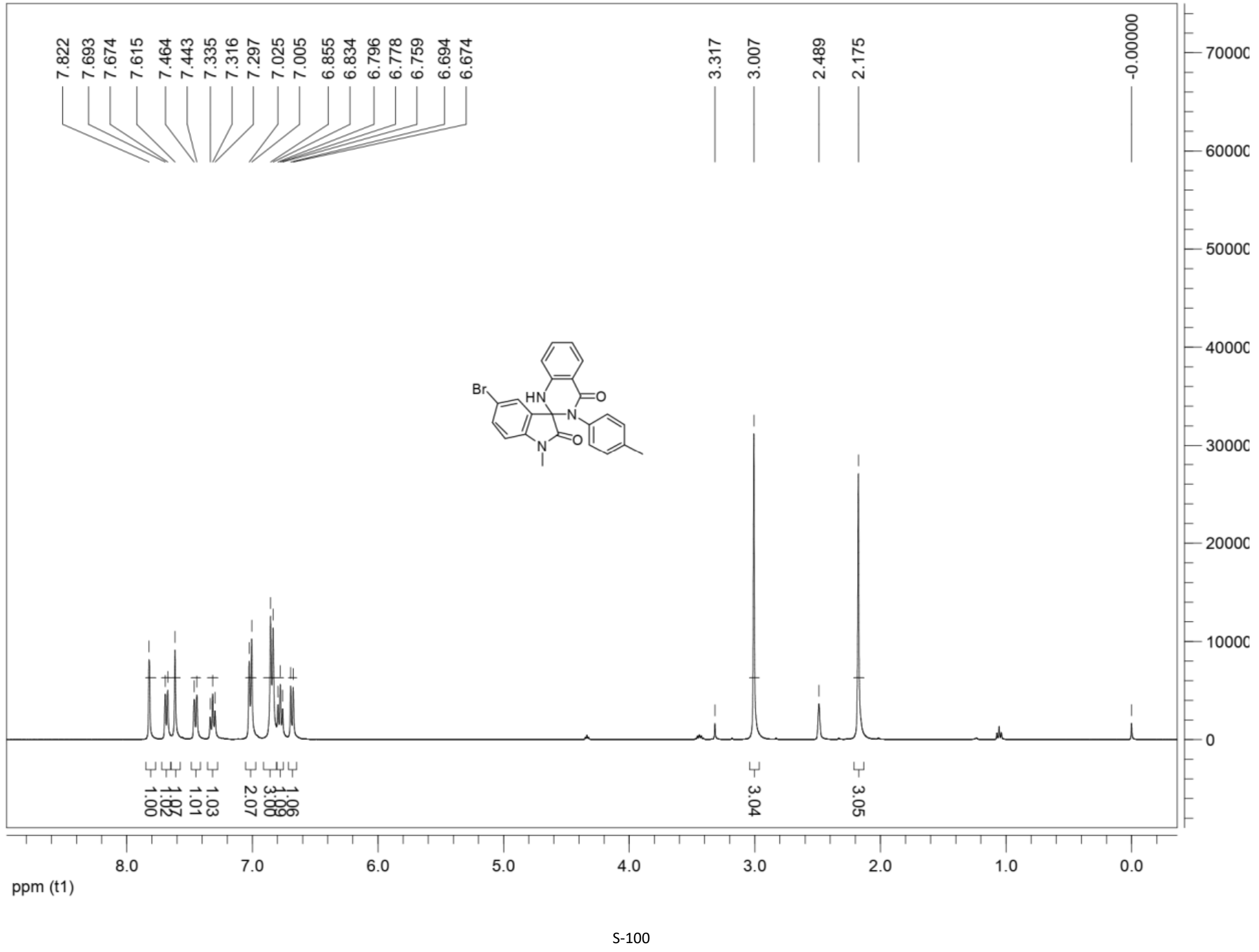


${ }^{13} \mathrm{C}$ NMR spectrum of Compound Bal (DMSO- $d_{6}, 100 \mathrm{MHz}$ )

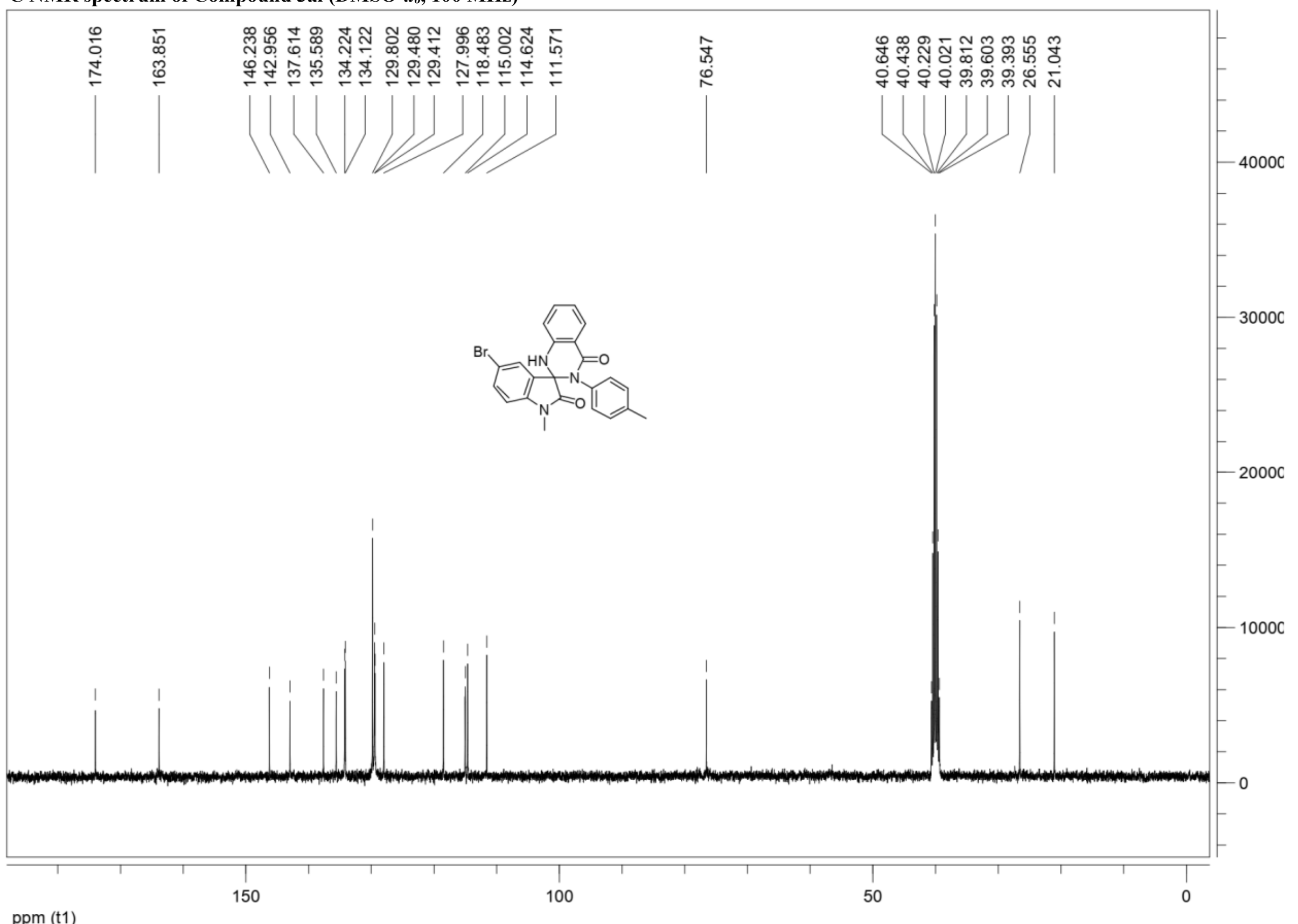

S-101 
8. Spectroscopic data for compounds 5, 7, 9, 11, 13, 16 and 18

${ }^{1} \mathrm{H}$ NMR spectrum of Compound 5 (DMSO- $\left.d_{6}, 400 \mathrm{MHz}\right)$

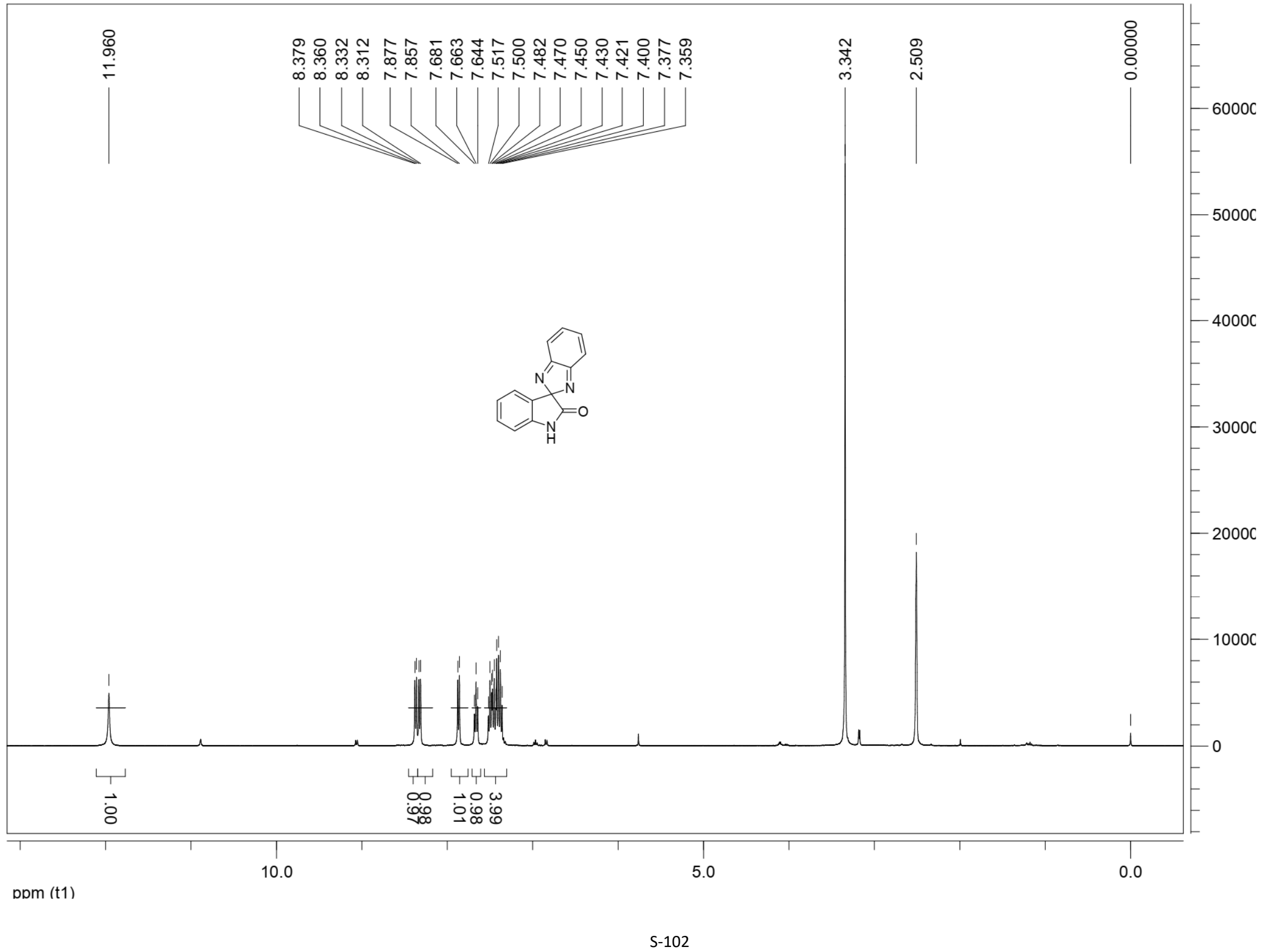


${ }^{13} \mathrm{C}$ NMR spectrum of Compound 5 (DMSO- $d_{6}, 100 \mathrm{MHz}$ )

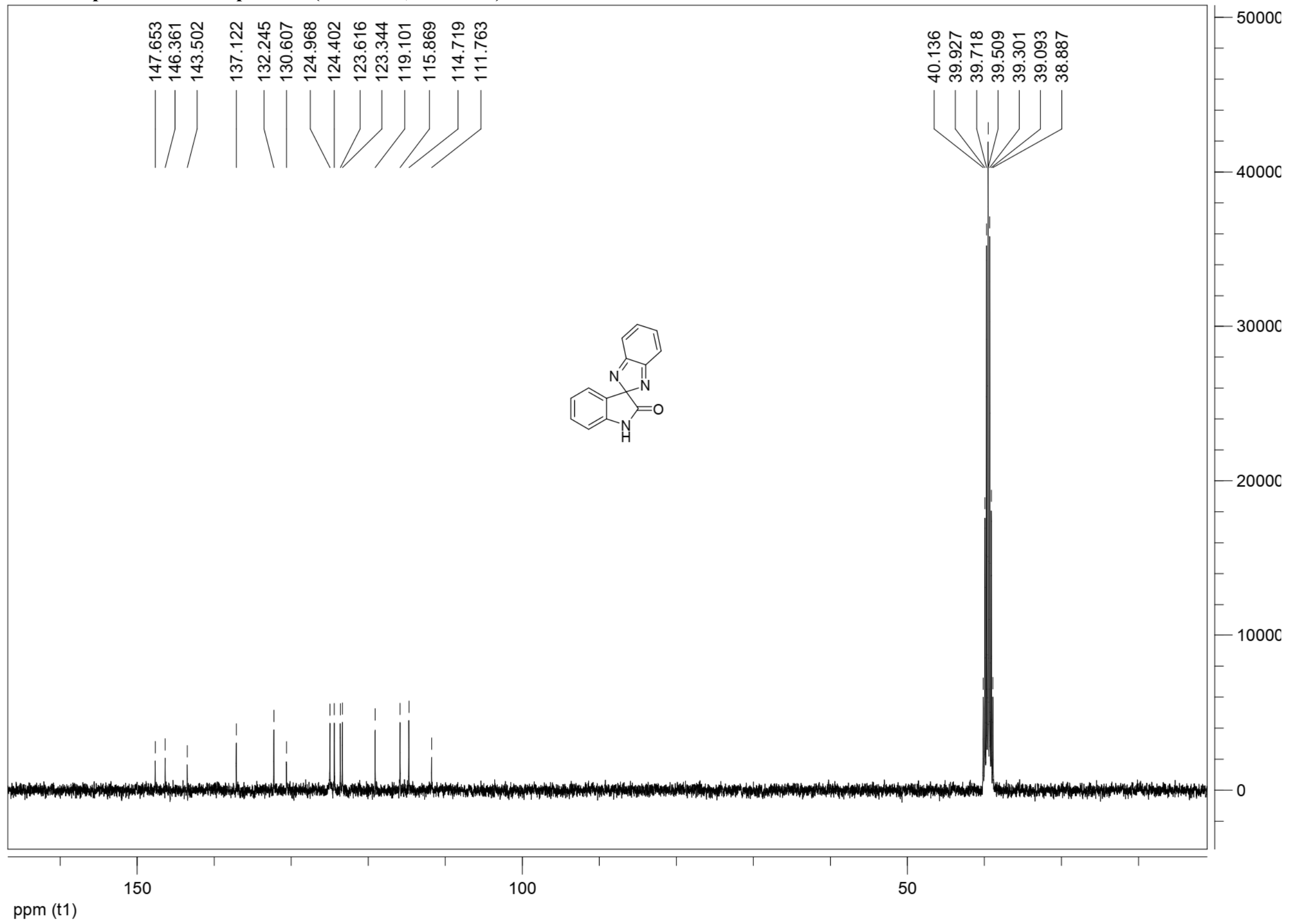


${ }^{1} \mathrm{H}$ NMR spectrum of Compound 7 (DMSO- $\left.d_{6}, 400 \mathrm{MHz}\right)$

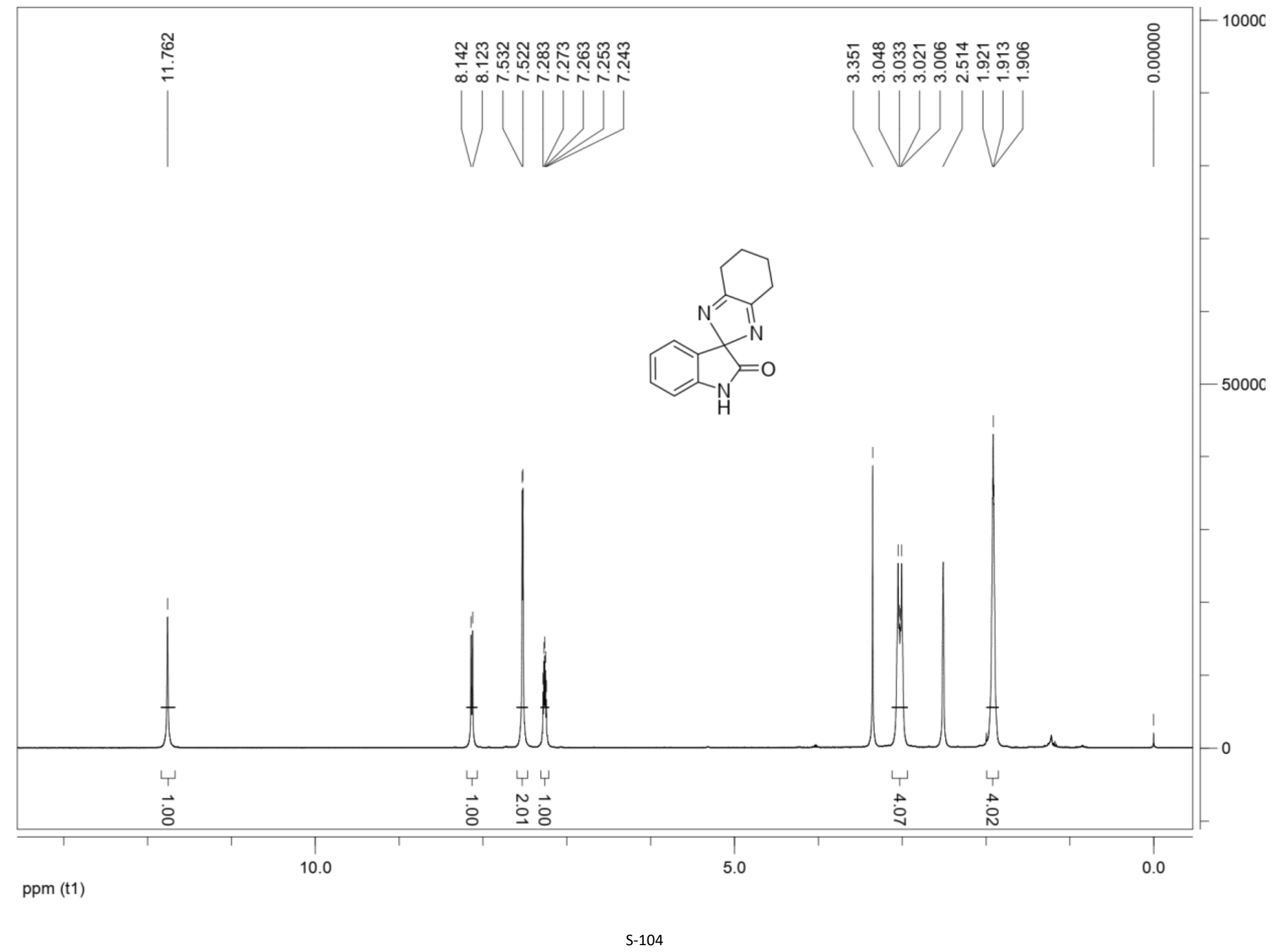


${ }^{13} \mathrm{C}$ NMR spectrum of Compound 7 (DMSO- $d_{6}, 100 \mathrm{MHz}$ )

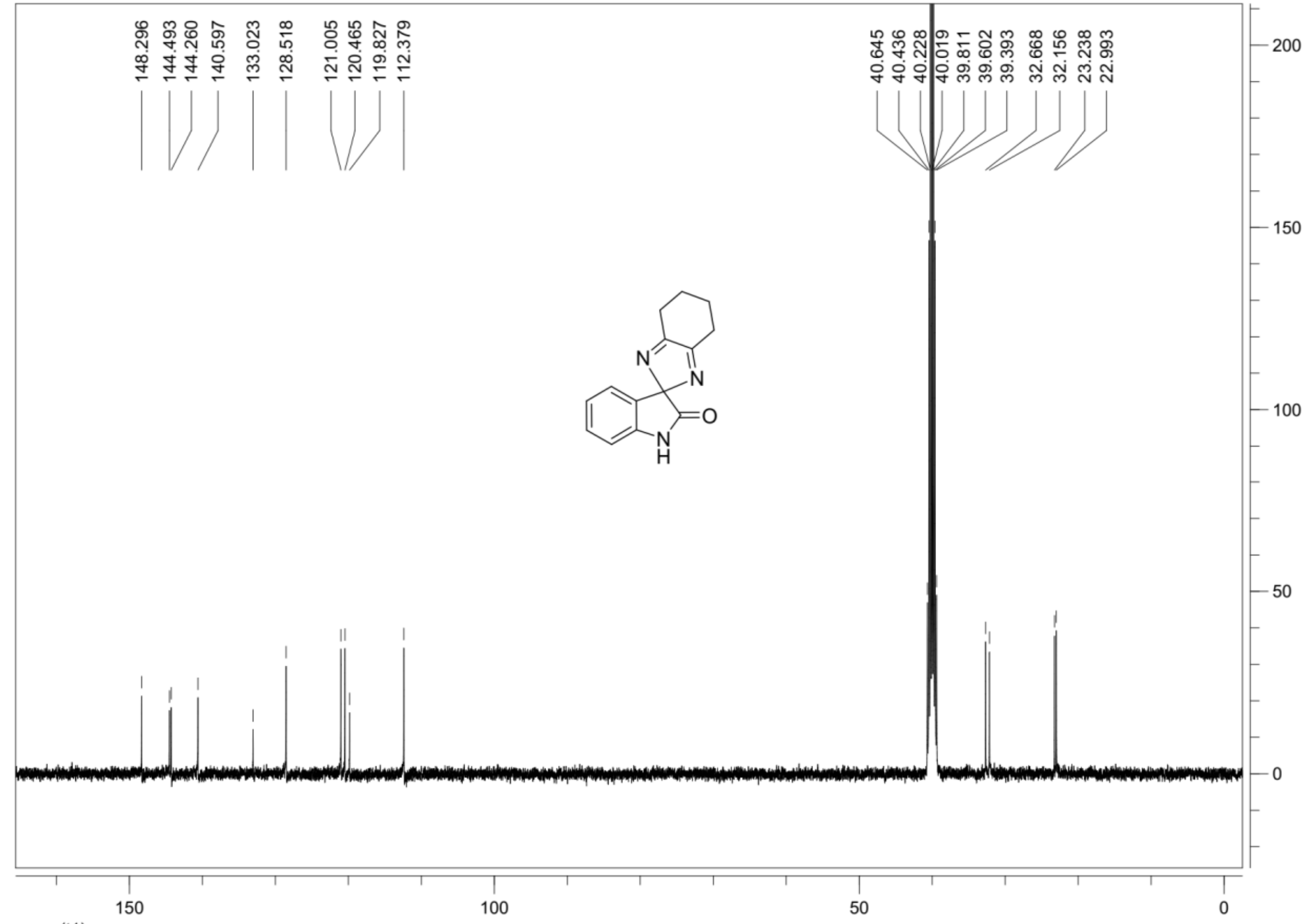

ppm (t1) 
${ }^{1} \mathrm{H}$ NMR spectrum of Compound 9 (DMSO- $\left.d_{6}, 400 \mathrm{MHz}\right)$

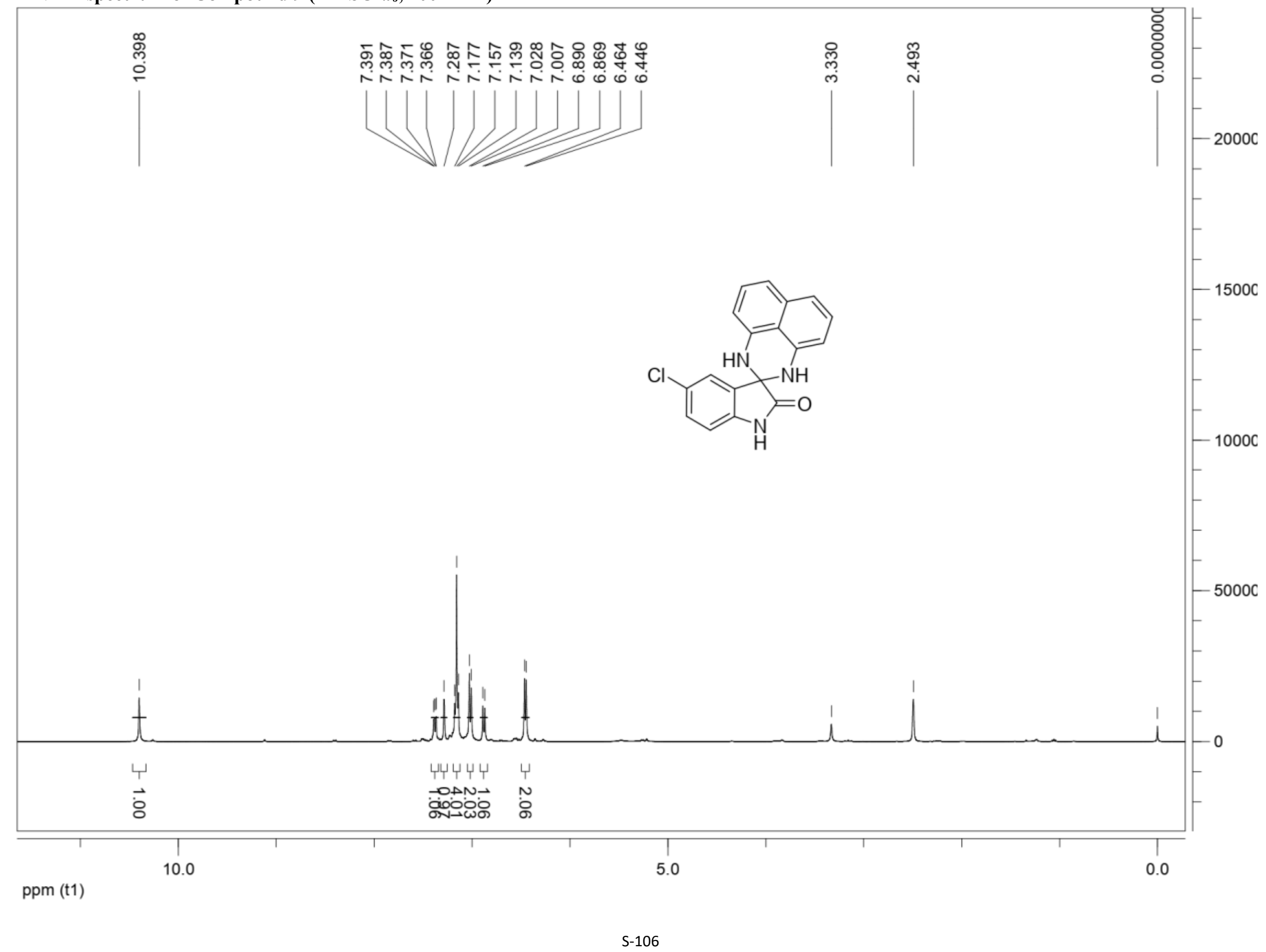


${ }^{13} \mathrm{C}$ NMR spectrum of Compound 9 (DMSO- $d_{6}, 100 \mathrm{MHz}$ )

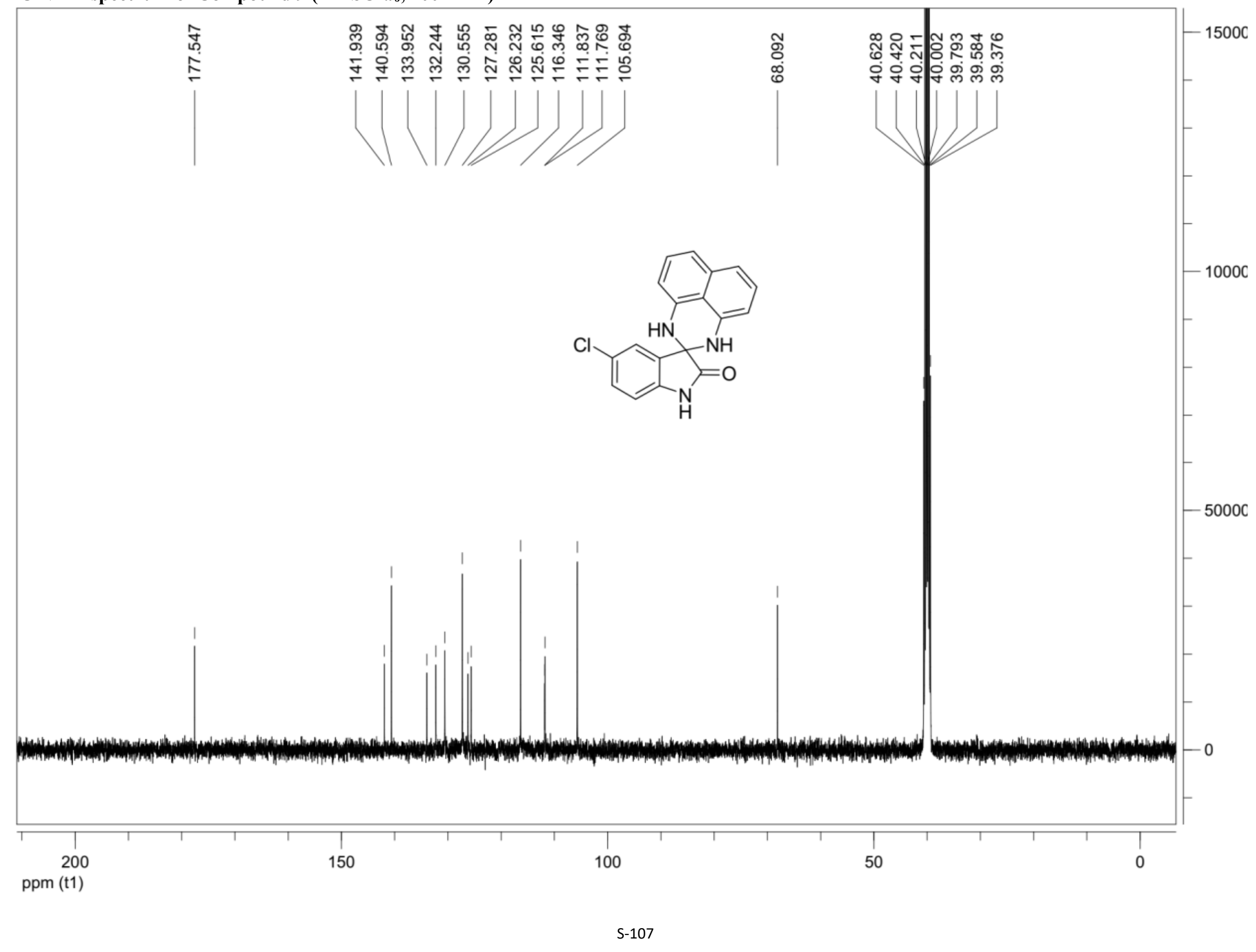




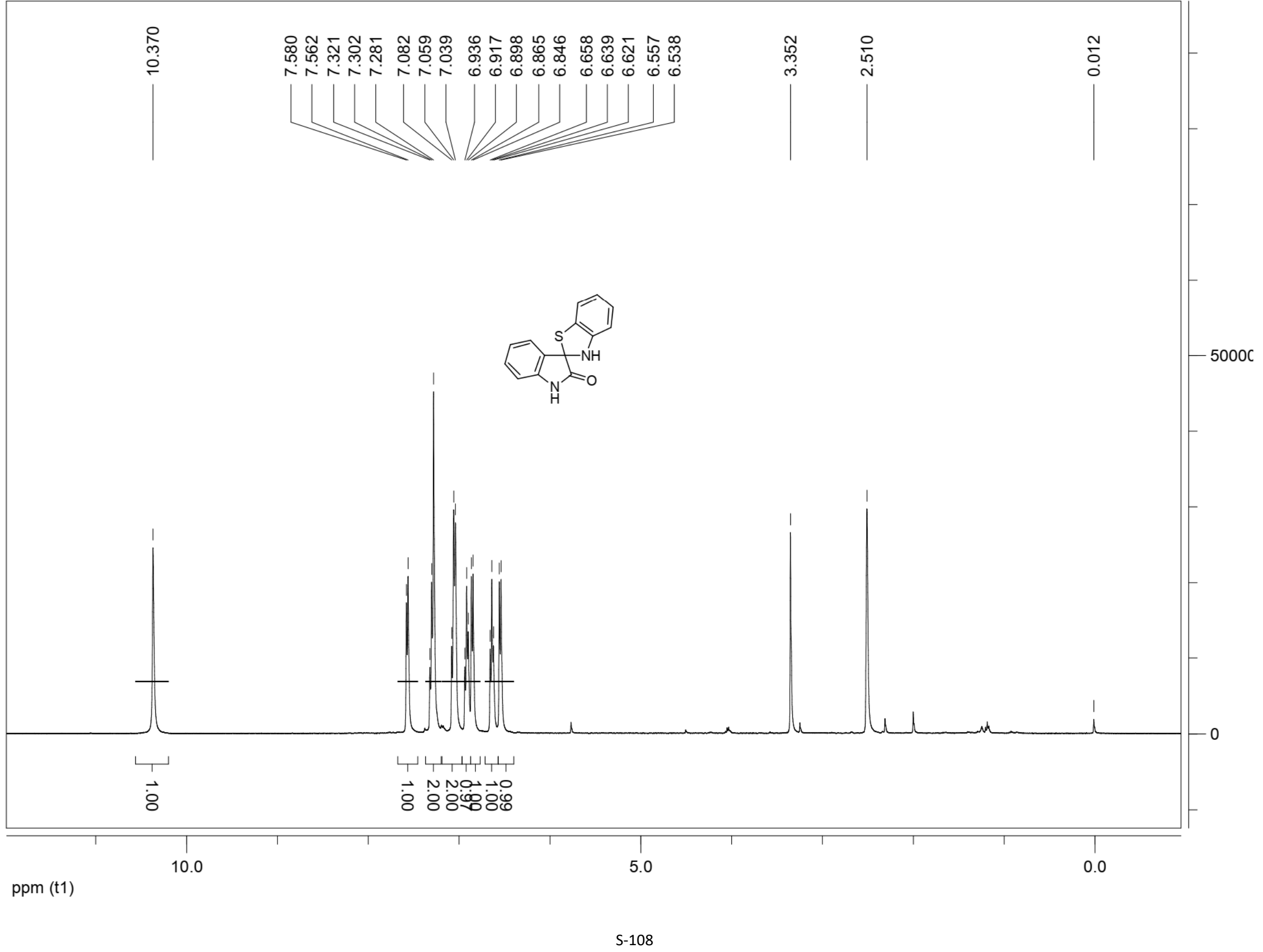




\section{${ }^{13} \mathrm{C}$ NMR spectrum of Compound 11 (DMSO- $d_{6}, 100 \mathrm{MHz}$ )}

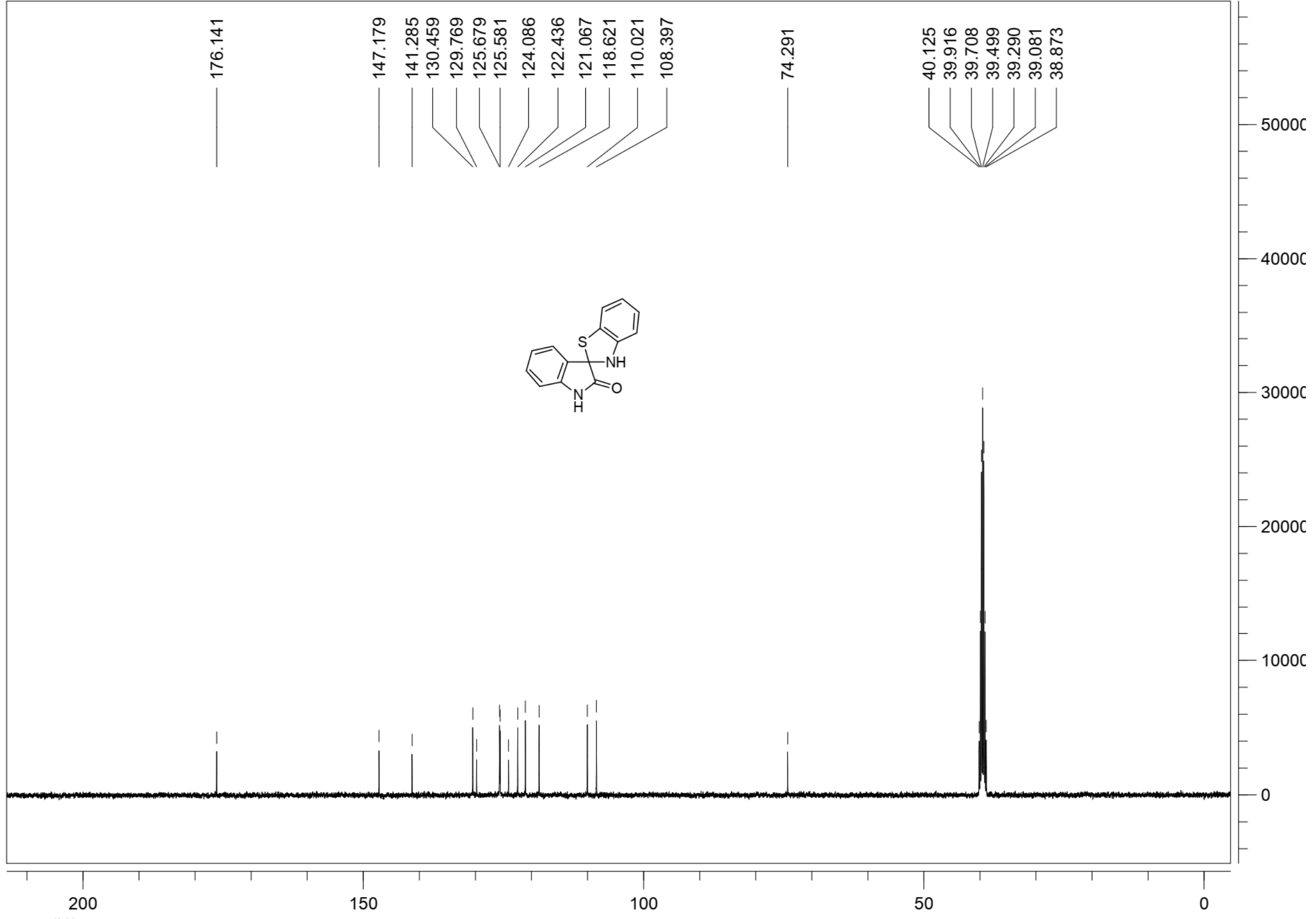

ppm (t1) 
${ }^{1} \mathrm{H}$ NMR spectrum of Compound 13 (DMSO- $d_{6}, 400 \mathrm{MHz}$ )

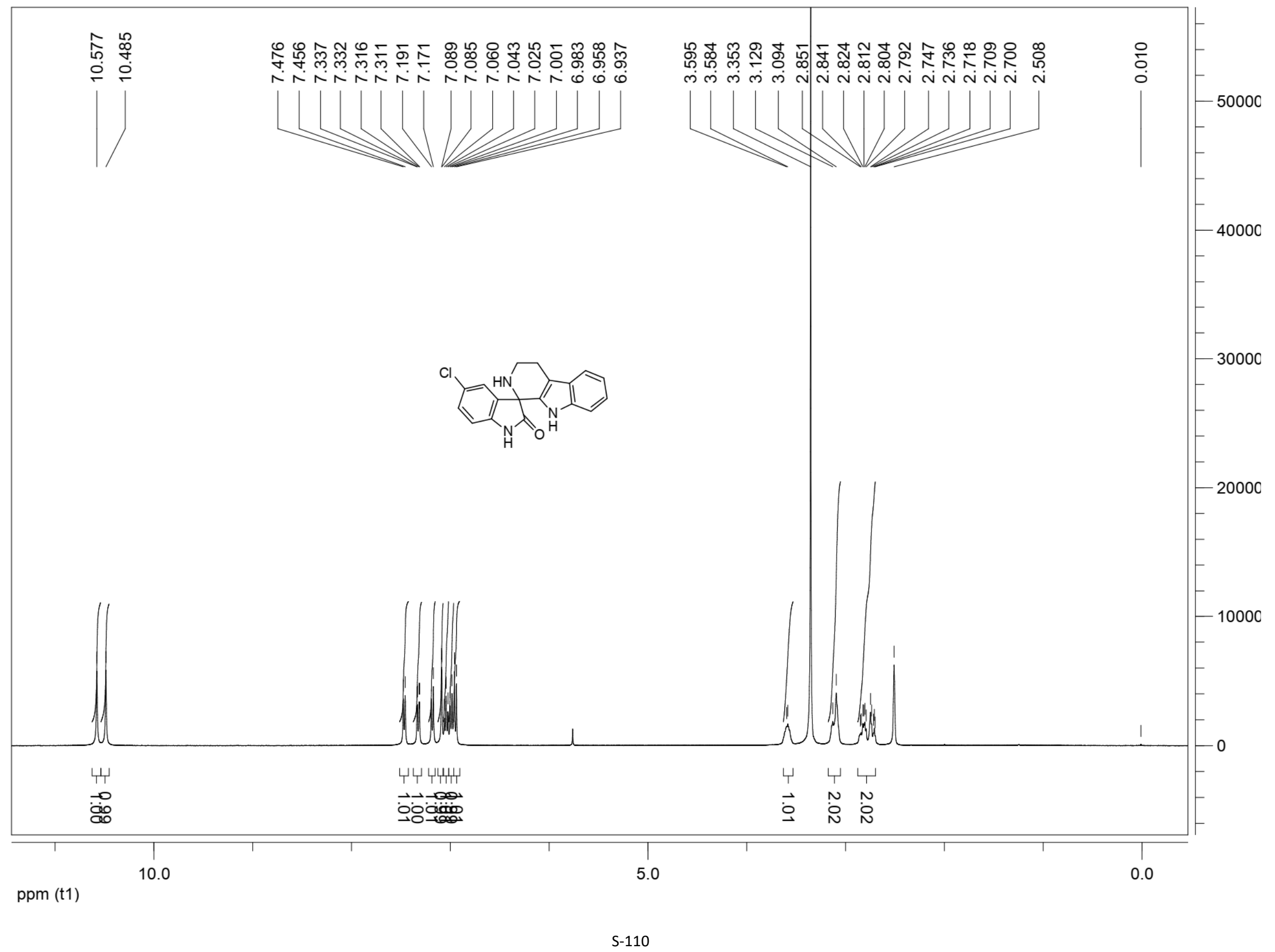




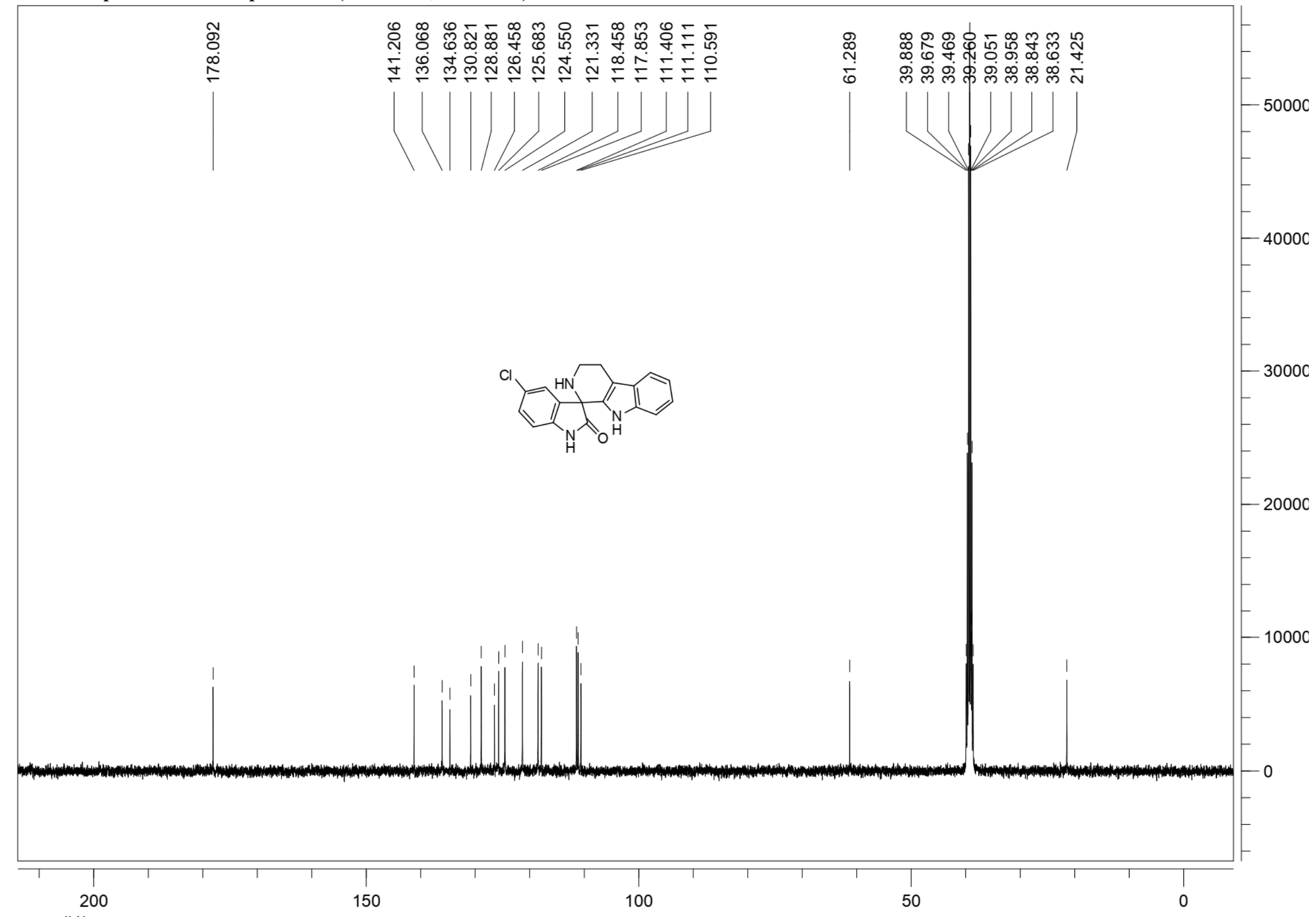

ppm (t1) 
${ }^{1} \mathrm{H}$ NMR spectrum of Compound 16 (DMSO- $\left.d_{6}, 400 \mathrm{MHz}\right)$

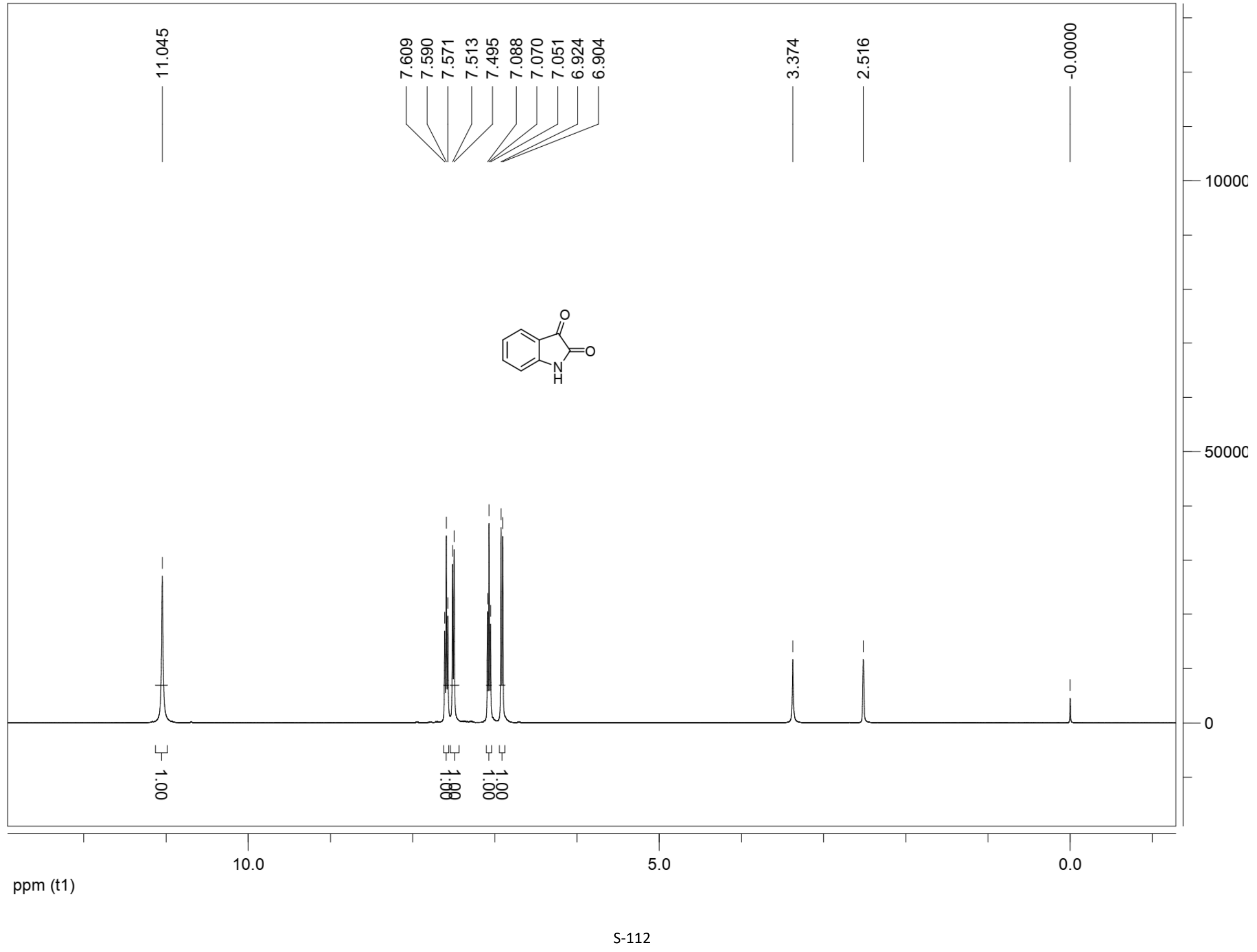


${ }^{13} \mathrm{C}$ NMR spectrum of Compound 16 (DMSO- $\left.d_{6}, 100 \mathrm{MHz}\right)$

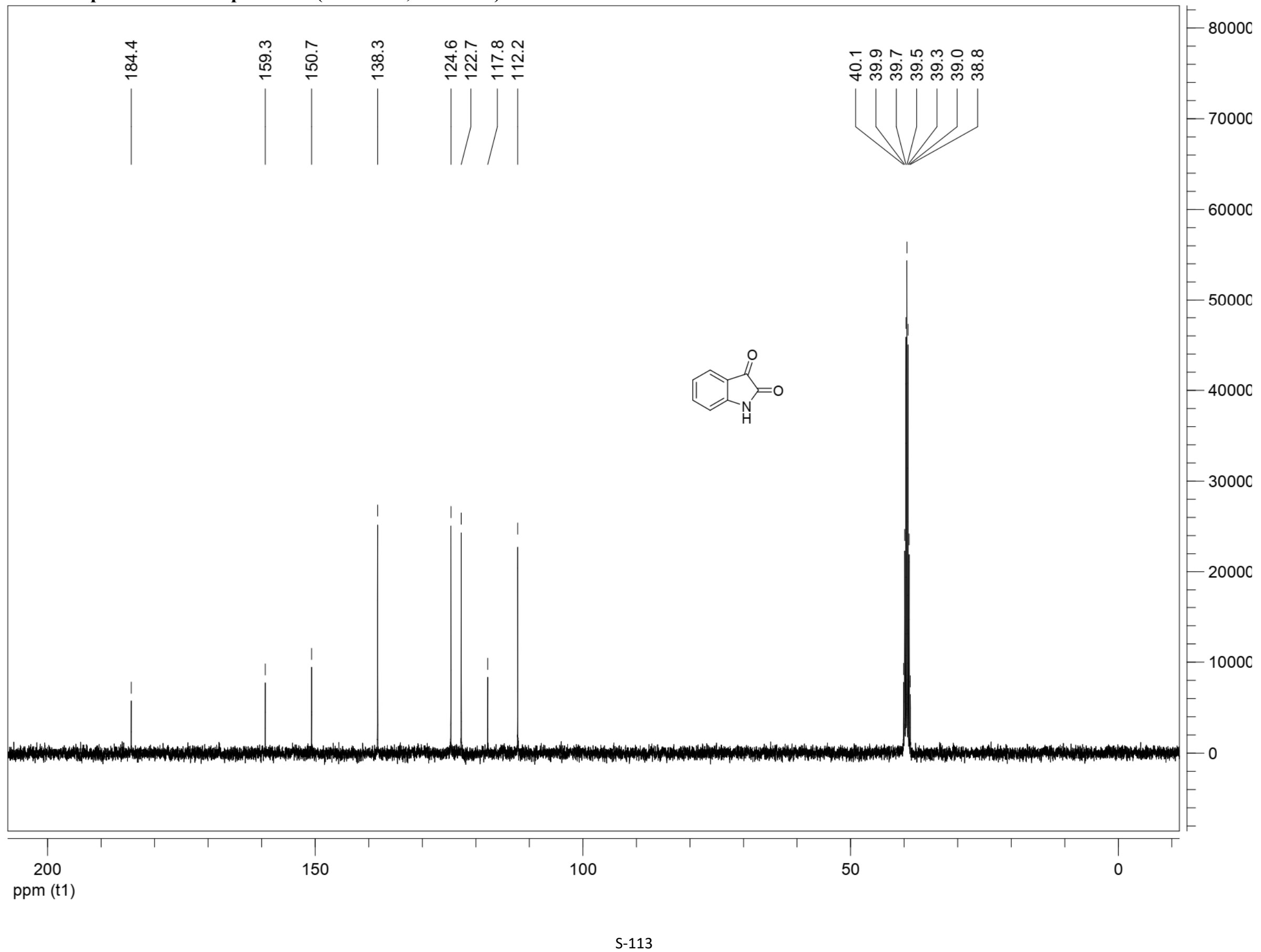


${ }^{1} \mathrm{H}$ NMR spectrum of Compound 18 (DMSO- $d_{6}, 400 \mathrm{MHz}$ )

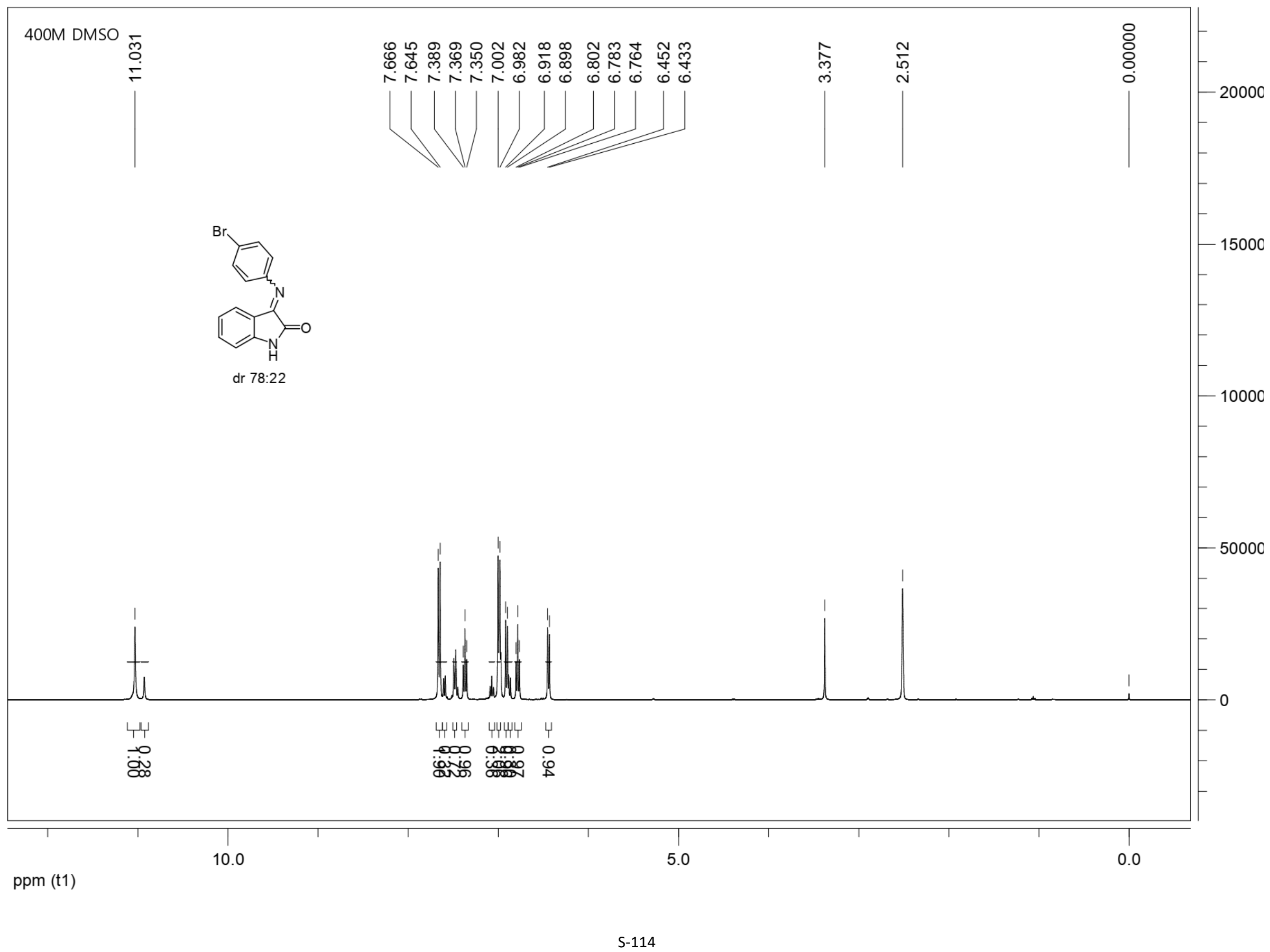


${ }^{13} \mathrm{C}$ NMR spectrum of Compound 18 (DMSO- $d_{6}, 100 \mathrm{MHz}$ )

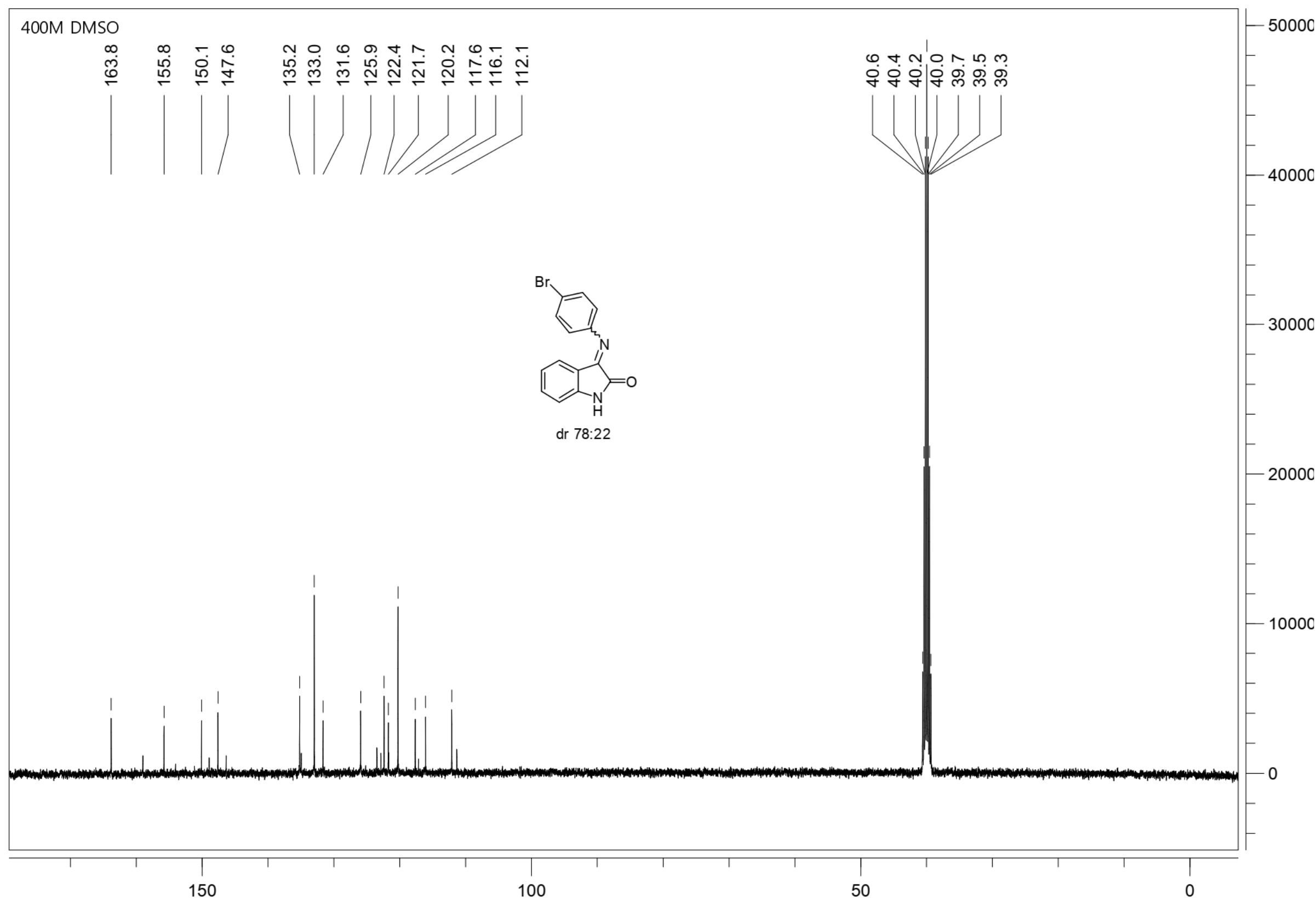

ppm (t1) 